Supporting Information

\title{
Access to Chiral GABA Analogues Bearing a Trifluoromethylated All-Carbon Quaternary Stereogenic Center through Water- Promoted Organocatalytic Michael Reactions
}

Jae Hun Sim,${ }^{\dagger}$ Jin Hyun Park, ${ }^{\dagger}$ Pintu Maity, and Choong Eui Song*

Department of Chemistry, Sungkyunkwan University, 2066, Seobu-ro, Jangan-gu, Suwon-si, Gyeonggi-do, 16419 (Korea); Fax: (+82) 31-290-7075

*E-mail: s1673@skku.edu 


\section{Table of contents}

1. General information $\quad \ldots \ldots \ldots \ldots . . . \quad S-3$

2. Optimization of the reaction conditions $\quad$........... S-4

2.1. Catalyst screening $\quad \ldots \ldots \ldots . . . \quad$ S-4

2.2. Pronucleophile screening $\quad \ldots \ldots \ldots . . . \quad S-5$

2.3. Miscellaneous reaction conditions $\quad$........... $\quad$ S-5

2.4. Control experiment for "on-water" effect $\quad$........... $\quad$ S-6

3. Synthesis of nitroolefins $\quad \ldots \ldots \ldots . . . \quad$ S-7

3.1. Synthetic procedures for nitroolefins $\quad \ldots \ldots \ldots . . . \quad$ S-7

3.2. Analytical data of nitroolefins $\quad$........... $\quad$ S-7

3.3. NMR spectra of nitroolefins $\quad$........... S-8

4. General procedure for the Michael reactions $\quad$........... S-11

4.1. General procedure for the non-enantioselective Michael reactions $\quad$.......... S-11

4.2. General procedure for the enantioselective Michael reactions $\quad$........... S-11

5. Synthesis of $\boldsymbol{\beta}-\mathrm{CF}_{3}$-GABA analogues $\quad \ldots \ldots \ldots . . . \quad$ S-12

6. Analytical data of the products (3 - 7) $\quad$........... S-13

6.1. NMR spectra of the products $(\mathbf{3}-\mathbf{7}) \quad \ldots \ldots \ldots . . . \quad$ S-27

6.2. HPLC spectra of the products $(\mathbf{3}-\mathbf{7}) \quad$.......... $\quad$ S-75

7. Single crystal X-ray structure analysis of ent-3ab (CCDC 1920284) $\quad$.......... S-111

8. References $\quad \ldots \ldots \ldots \ldots . . . \quad$ S-113 


\section{General information}

- Chemical : Chemicals were purchased from commercial vendors (e.g., Aldrich, Alfa Aesar, TCI) and used without further purification. $\beta$-Trifluoromethyl $\left(\mathrm{CF}_{3}\right)-\alpha$-nitroolefins and $\beta$-perfluoroalkyl $\left(\mathrm{CHF}_{2}, \mathrm{C}_{2} \mathrm{~F}_{5}, \mathrm{C}_{3} \mathrm{~F}_{7}\right)$ - $\alpha$-nitroolefins (hereinafter referred to as $\beta-\mathrm{R}_{\mathrm{F}}-\alpha$-nitroolefins) were synthesized according to the reported procedures ${ }^{1}$. Dithiomalonates (DTMs) were synthesized according to the reported procedure ${ }^{2}$. Catalysts, examined in this study, were synthesized according to the reported procedures ${ }^{3}$.

- Solvent : HPLC grade solvents, anhydrous solvents, NMR solvents and additional organic solvents were purchased from commercial vendors (e.g., Aldrich, Alfa Aesar, CIL Inc., Merck) and used without further distillation or purification.

- Reaction : Reaction mixtures were stirred magnetically in flame-dried glassware (e.g., roundbottom flask (RBF), vial) under Ar gas atmosphere. Yields refer to spectroscopically pure products unless otherwise stated.

- Thin Layer Chromatography (TLC) : TLC results were performed using silica gel plates (Merck, Kieselgel 60 F254 $0.25 \mathrm{~mm}$ ).

- Column chromatographic purification : Purification processes were performed by using silica gel (Merck, $60 \AA$ А, 230-400 mesh, 0.040-0.063 mm).

- Nuclear Magnetic Resonance (NMR) spectroscopy : ${ }^{1} \mathrm{H}$ NMR (500 MHz), ${ }^{13} \mathrm{C}$ NMR $(125.7 \mathrm{MHz})$ and ${ }^{19} \mathrm{~F}$ NMR (470.4 MHz) spectra were recorded using Bruker Ascend ${ }^{\mathrm{TM}} 500$ or Varian Unity INOVA 500 spectrometer. Chemical shifts were reported by using tetramethylsilane (TMS) as the internal standard for ${ }^{1} \mathrm{H}$ and ${ }^{13} \mathrm{C}$ NMR, and benzotrifluoride $\left(\mathrm{C}_{6} \mathrm{H}_{5} \mathrm{CF}_{3}\right)$ as the external standard for ${ }^{19} \mathrm{~F}$ NMR. For ${ }^{13} \mathrm{C}$ NMR spectra using $\mathrm{D}_{2} \mathrm{O}$ as the NMR solvent, 1,4-dioxane or $\mathrm{CD}_{3} \mathrm{OD}$ was used as the internal standard (1,4-dioxane: $\left.67.19 \mathrm{ppm} ; \mathrm{CD}_{3} \mathrm{OD}: 49.50 \mathrm{ppm}\right)^{4}$.

- High Performance Liquid Chromatography (HPLC) : HPLC analysis were performed on Varian Pro Star Series or YL9100 Plus HPLC System instrument equipped with an isostatic pump using chiral column (CHIRALCEL OJ-H, CHIRALPAK AD-H, CHIRALPAK ${ }^{\circledR}$ IA, CHIRALPAK ${ }^{\circledR}$ IB; $250 \times 4.6$ $\mathrm{mm}$ ) for the determination of the enantiomeric excess $(e e)$ of the products.

- Mass Spectroscopy (MS) : High-resolution mass spectra (HR-MS) were performed by using Bruker Compact spectrometer.

- Infrared (IR) spectroscopy : IR spectra were recorded using Bruker Vertex 70 spectrometer with MIRacle Micro ATR accessory.

- Specific rotation $\left([\boldsymbol{\alpha}]_{\mathbf{D}}{ }^{\mathbf{T}}\right):$ Specific rotations were measured by using PerkinElmer 343 plus polarimeter.

- Melting point : Melting points were measured by using Büchi ${ }^{\circledR}$ B-540 apparatus.

- X-ray crystallography : Single crystal X-ray diffraction (SC-XRD) was analyzed by using Bruker D8 diffractometer system. 


\section{Optimization of the reaction conditions}

\subsection{Catalyst screening ${ }^{a, b, c}$}

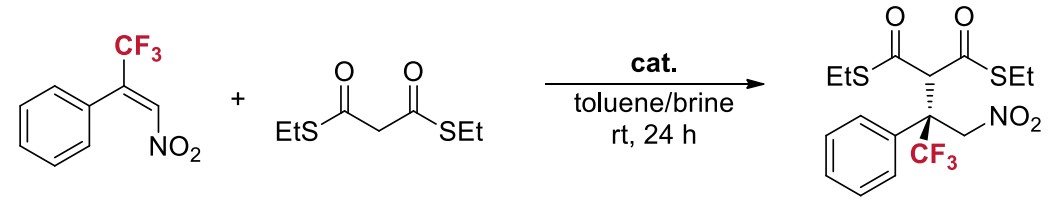

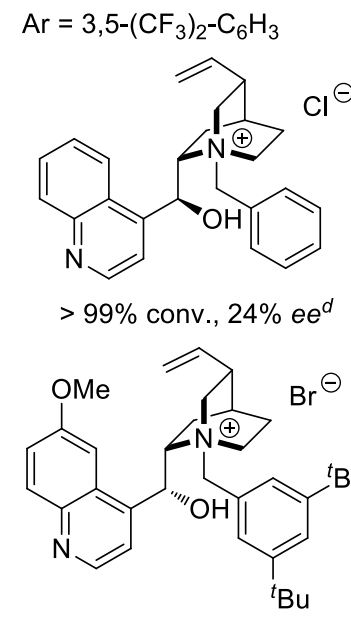

$86 \%$ conv., $10 \%$ ee<smiles>C=CC1CN2CCC1C2[C@H](O)c1ccnc2ccc(OC)cc12</smiles>

n.r. ${ }^{e}$<smiles>[Z]C(N[Al])N[C@@H]1CCCC[C@H]1N(C)C</smiles>

$(Z=0): 19 \%$ conv., $89 \%$ ee $(Z=S): 11 \%$ conv., $94 \%$ ee

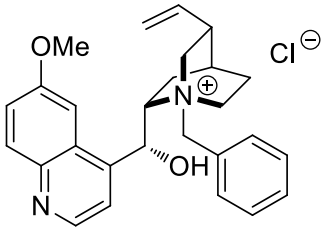

$82 \%$ conv., $7 \%$ ee

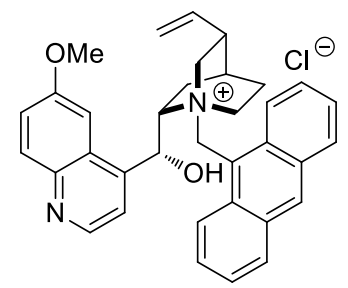

$78 \%$ conv., $23 \%$ ee

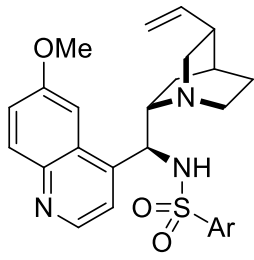

n.r. . $^{-}$<smiles>CCN[C@@H]1CCCC[C@H]1NC(=O)N[Al]</smiles>

$49 \%$ conv., $84 \%$ ee

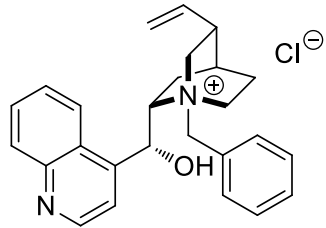

$90 \%$ conv., $28 \%$ ee

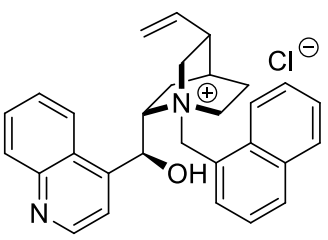

$89 \%$ conv., $35 \%$ ee

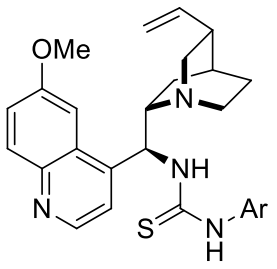

n.r. ${ }^{e}$

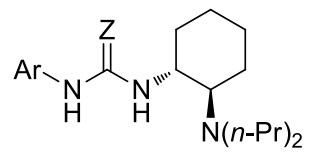

$(Z=0): 10 \%$ conv., $82 \%$ ee $(Z=S): 10 \%$ conv., $80 \%$ ee

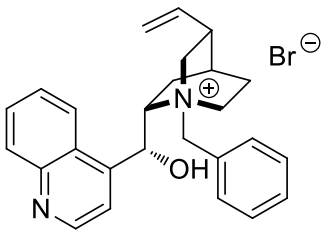

$91 \%$ conv., $23 \%$ ee

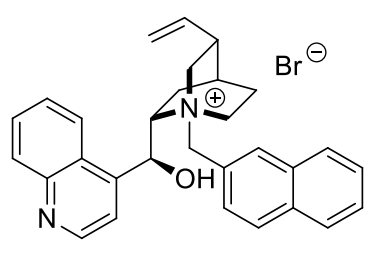

$79 \%$ conv., $31 \%$ ee
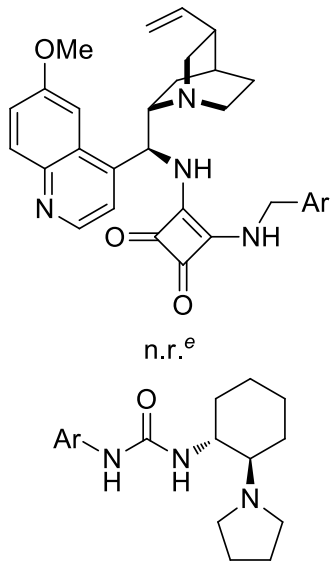

$52 \%$ conv., $94 \%$ ee<smiles>Brc1cc2ccccc2c2c1C[N@+]1(Cc3ccc4ccccc4c3)Cc3ccc4ccccc4c3-c3c(ccc4ccccc34)C[NH+]2C1</smiles>

$94 \%$ conv., $36 \%$ ee

Scheme S1. ${ }^{a}$ Reaction condition: 1a $(0.1 \mathrm{mmol})$, 2a (1.2 equiv., $\left.0.12 \mathrm{mmol}\right)$, catalyst $(10 \mathrm{~mol} \%, 0.01$ mmol), toluene (HPLC grade, 5 equiv., $0.5 \mathrm{mmol}$ ), brine (sat., $2 \mathrm{~mL}$ ), rt, $24 \mathrm{~h} .{ }^{b}$ The conversion was determined by ${ }^{19} \mathrm{~F}$ NMR integration. ${ }^{c}$ The $\%$ ee was determined by HPLC analysis using a chiral stationary phase. ${ }^{d} 48 \mathrm{~h}$ reaction. ${ }^{e}$ n.r. $=$ no reaction 


\subsection{Pronucleophile screening ${ }^{a, b, c}$}

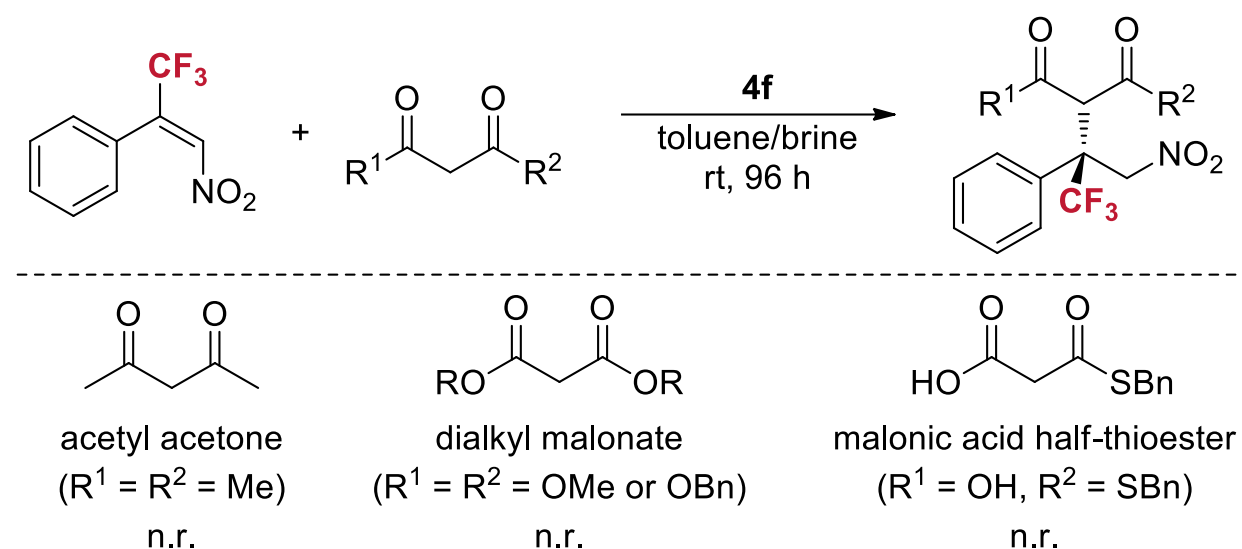

Scheme S2. ${ }^{a}$ Reaction condition: 1a $(0.1 \mathrm{mmol})$, pro-nucleophile ( 2 equiv., $\left.0.2 \mathrm{mmol}\right), \mathbf{4 f}(0.15$ equiv., $0.015 \mathrm{mmol}$ ), toluene (HPLC grade, 5 equiv., $0.5 \mathrm{mmol}$ ), brine (sat., $2 \mathrm{~mL}$ ), rt, $96 \mathrm{~h} .{ }^{b}$ The conversion was determined by ${ }^{19} \mathrm{~F}$ NMR integration. ${ }^{c}$ n.r. $=$ no reaction.

\subsection{Miscellaneous reaction conditions ${ }^{a, b, c}$}
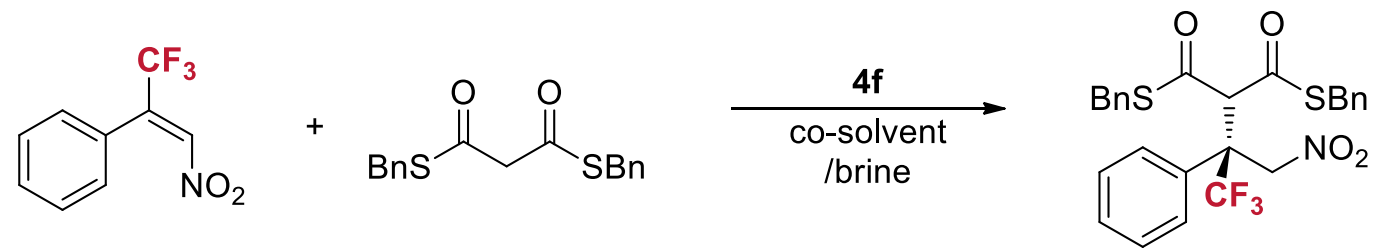

\begin{tabular}{|c|c|c|c|c|c|c|c|}
\hline Entry & $\begin{array}{c}\text { Cat. } \\
(\mathbf{m o l} \%)\end{array}$ & $\begin{array}{c}\text { DTM } \\
\text { (equiv.) }\end{array}$ & Co-solvent & $\begin{array}{l}\text { Temp. } \\
\left({ }^{\circ} \mathrm{C}\right)\end{array}$ & $\begin{array}{l}\text { Time } \\
\text { (h) }\end{array}$ & $\begin{array}{c}\text { Conv. } \\
(\%)\end{array}$ & $\begin{array}{c}e e \\
(\%)\end{array}$ \\
\hline $1^{d}$ & 20 & 1.2 & - & $\mathrm{rt}$ & 48 & 93 & 92 \\
\hline $2^{d}$ & 20 & 1.2 & cyclohexane & $\mathrm{rt}$ & 48 & 92 & 93 \\
\hline $3^{d}$ & 20 & 1.2 & hexane & $\mathrm{rt}$ & 48 & 86 & 93 \\
\hline $4^{d}$ & 20 & 1.2 & $o$-xylene & $\mathrm{rt}$ & 48 & 81 & 94 \\
\hline $5^{d}$ & 20 & 3.0 & toluene & $\mathrm{rt}$ & 48 & 72 & 94 \\
\hline $6^{d}$ & 20 & 3.0 & toluene & $\mathrm{rt}$ & 96 & 80 & 94 \\
\hline 7 & 15 & 1.5 & cyclohexane & $\mathrm{rt}$ & 36 & 91 & 93 \\
\hline 8 & 15 & 1.5 & cyclohexane & $\mathrm{rt}$ & 48 & 92 & 92 \\
\hline 9 & 15 & 1.5 & cyclohexane & $\mathrm{rt}$ & 72 & 94 & 94 \\
\hline 10 & 15 & 1.5 & cyclohexane & $\mathrm{rt}$ & 96 & 96 & 94 \\
\hline 11 & 15 & 1.5 & cyclohexane & 0 & 96 & 96 & 96 \\
\hline $12^{e}$ & 15 & 2.0 & cyclohexane & 0 & 96 & 92 & 95 \\
\hline $13^{f}$ & 15 & 2.0 & cyclohexane & 0 & 96 & 92 & 95 \\
\hline 14 & 15 & 2.0 & methylcyclohexane & 0 & 96 & 92 & 95 \\
\hline
\end{tabular}

Table S1. ${ }^{a}$ Reaction condition: 1a $(0.1 \mathrm{mmol}), \mathbf{2 b}, \mathbf{4 f}(0.15$ equiv., $0.015 \mathrm{mmol})$, co-solvent (HPLC grade, 5 equiv., $0.5 \mathrm{mmol}$ ), brine (sat., $2 \mathrm{~mL}$ ). ${ }^{b}$ The conversion was determined by ${ }^{19} \mathrm{~F}$ NMR integration. ${ }^{c}$ The $\%$ ee was determined by HPLC analysis using a chiral stationary phase. ${ }^{d} \mathbf{2 a}$ was used. ${ }^{e}(10$ equiv., $1 \mathrm{mmol})$ co-solvent was used. ${ }^{f}(15$ equiv., $1.5 \mathrm{mmol})$ co-solvent was used. 


\subsection{Control experiment for "on-water" effect ${ }^{a, b, c}$}

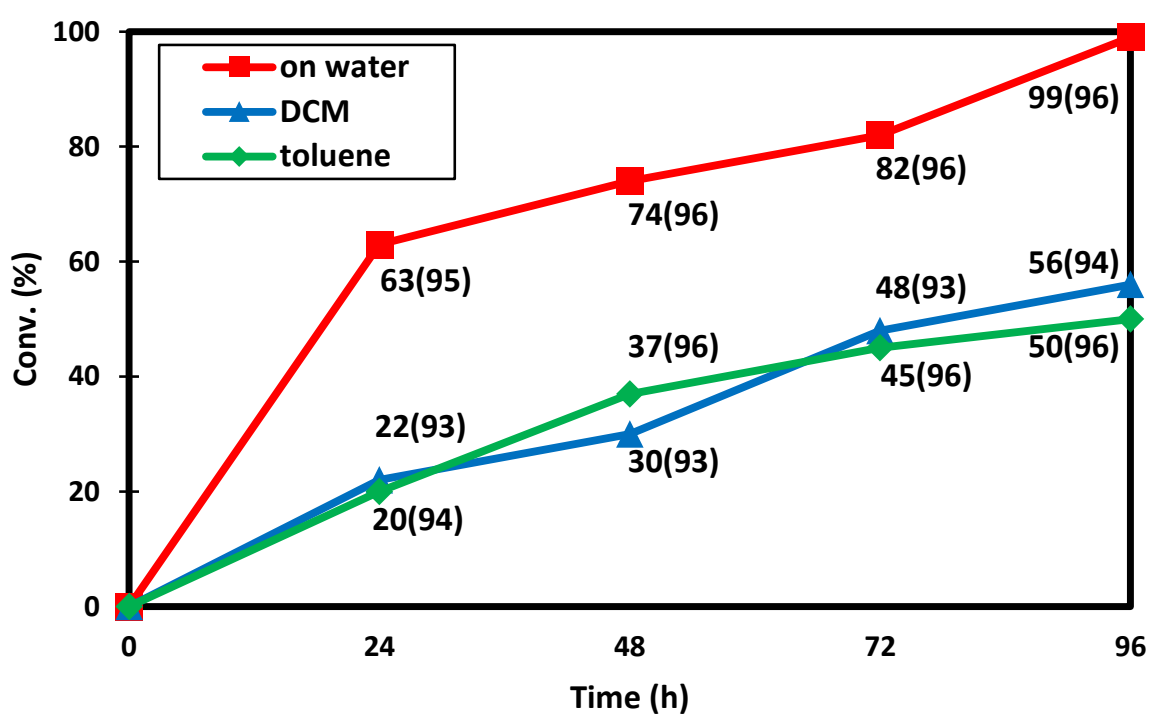

Figure S1. ${ }^{a}$ Reaction condition: $1 \mathbf{a}(0.03 \mathrm{mmol}), \mathbf{2 b}$ ( 2 equiv., $\left.0.06 \mathrm{mmol}\right), \mathbf{4 f}(15 \mathrm{~mol} \%, 0.0045$ $\mathrm{mmol}$ ), toluene (HPLC grade, 5 equiv., $0.15 \mathrm{mmol}$ ) and brine (sat., $0.4 \mathrm{~mL}$ ), or organic solvent (HPLC grade, $0.1 \mathrm{M}, 0.3 \mathrm{~mL}$ ), $0{ }^{\circ} \mathrm{C} .{ }^{b}$ The conversion was determined by ${ }^{19} \mathrm{~F}$ NMR integration (values on points). ${ }^{\circ}$ The $\%$ ee was determined by HPLC analysis using a chiral stationary phase (values in parentheses). 


\section{Synthesis of nitroolefins}

\subsection{Synthetic procedures for nitroolefins}

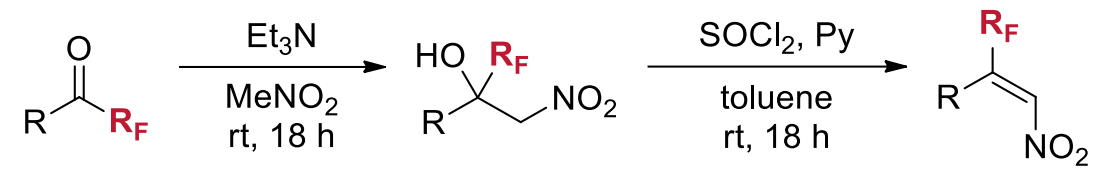

To the mixture of $\mathrm{R}_{\mathrm{F}}$-ketone $(10 \mathrm{mmol})$ and $\mathrm{MeNO}_{2}(0.5 \mathrm{M}, 20 \mathrm{~mL})$ was added $\mathrm{Et}_{3} \mathrm{~N}$ (1.5 equiv., 15 mmol) dropwise. The reaction mixture was stirred at $\mathrm{rt}$ for $18 \mathrm{~h}$, after then diluted with EtOAc and washed with $1 \mathrm{~N} \mathrm{HCl}$ successively. The organic layer was extracted, and dried over anhydrous $\mathrm{Na}_{2} \mathrm{SO}_{4}$, and filtered. The filtrate was concentrated in vасиo, and the residue was purified by column chromatography on silica gel (EtOAc:hexanes $=1: 50$ to 1:10) to afford corresponding $\mathrm{R}_{\mathrm{F}}$-nitroalcohol.

To the mixture of $\mathrm{R}_{\mathrm{F}}$-nitroalcohol in toluene (anhydrous, $0.25 \mathrm{M}$ ) were added $\mathrm{SOCl}_{2}$ (1.5 equiv.) and pyridine ( 2 equiv.) continuously at $0{ }^{\circ} \mathrm{C}$. The reaction mixture was stirred at $\mathrm{rt}$ for $18 \mathrm{~h}$, after then diluted with EtOAc and washed with brine (sat.) successively. The organic layer was extracted, and dried over anhydrous $\mathrm{Na}_{2} \mathrm{SO}_{4}$, and filtered. The filtrate was concentrated in vacuo, and the residue was purified by column chromatography on silica gel (EtOAc:hexanes $=1: 50$ to 1:10) to afford corresponding $\beta$ - $\mathrm{R}_{\mathrm{F}-} \alpha$-nitroolefin (1).

* All the NMR spectra data of known compounds were in accordance with the reported references ${ }^{1}$, and the analytical data of new compounds are indicated as follows.

\subsection{Analytical data of nitroolefins}<smiles>COc1ccc(/C(=C\[N+](=O)[O-])C(F)(F)F)cc1OC1CCCC1</smiles>

1o, New Compound, (yellow solid, 75\% yield, $2.4847 \mathrm{~g}$ ).

$\mathbf{R}_{\mathbf{f}}=0.60$ (acetone:hexanes, $1: 10 \mathrm{v} / \mathrm{v}$ ).

mp: $58^{\circ} \mathrm{C}$.

${ }^{1} \mathbf{H}$ NMR $\left(500 \mathrm{MHz}, \mathrm{CDCl}_{3}, \mathrm{Me}_{4} \mathrm{Si}\right): \delta 7.46(\mathrm{~s}, 1 \mathrm{H}), 6.90-6.86(\mathrm{~m}, 2 \mathrm{H})$, $6.79(\mathrm{~s}, 1 \mathrm{H}), 4.76-4.72(\mathrm{~m}, 1 \mathrm{H}), 3.87(\mathrm{~s}, 3 \mathrm{H}), 1.94-1.81(\mathrm{~m}, 6 \mathrm{H}), 1.64-1.60(\mathrm{~m}, 2 \mathrm{H})$.

${ }^{13} \mathrm{C} \mathrm{NMR}\left(126 \mathrm{MHz}, \mathrm{CDCl}_{3}, \mathrm{Me}_{4} \mathrm{Si}\right): \delta 152.02,147.82,139.37\left(\mathrm{q},{ }^{3} J_{\mathrm{C}-\mathrm{F}}=6.1 \mathrm{~Hz}\right), 135.46\left(\mathrm{q},{ }^{2} J_{\mathrm{C}-\mathrm{F}}=\right.$ $31.6 \mathrm{~Hz}), 122.07\left(\mathrm{q},{ }^{1} J_{\mathrm{C}-\mathrm{F}}=275.7 \mathrm{~Hz}\right), 121.14,118.56,114.63,111.77,80.70,55.98,32.68,24.09$.

${ }^{19} \mathbf{F ~ N M R ~}\left(470 \mathrm{MHz}, \mathrm{CDCl}_{3}\right): \delta-66.40(\mathrm{~s}, 3 \mathrm{~F})$.

IR: $v$ 2962, 2928, 2855, 2355, 2343, 1543, 1516, 1259, $1141 \mathrm{~cm}^{-1}$.

HR-MS (ESI): $\mathrm{m} / \mathrm{z}$ calcd. for $\left[\mathrm{C}_{15} \mathrm{H}_{16} \mathrm{~F}_{3} \mathrm{NO}_{4}+\mathrm{Na}\right]^{+}:$354.0924; found, 354.0926.<smiles>O=[N+]([O-])C=C(c1ccccc1)C(F)(F)F</smiles>

1v, New Compound, (yellow liquid, $72 \%$ yield, $1.9235 \mathrm{~g}$ ).

$\mathbf{R}_{\mathbf{f}}=0.43$ (acetone:hexanes, $1: 10 \mathrm{v} / \mathrm{v}$ ).

${ }^{1} \mathbf{H}$ NMR $\left(500 \mathrm{MHz}, \mathrm{CDCl}_{3}, \mathrm{Me}_{4} \mathrm{Si}\right): \delta 7.51-7.38(\mathrm{~m}, 4 \mathrm{H}), 7.26(\mathrm{~m}, 2 \mathrm{H})$.

${ }^{13} \mathrm{C}$ NMR $\left(126 \mathrm{MHz}, \mathrm{CDCl}_{3}, \mathrm{Me}_{4} \mathrm{Si}\right): \delta 142.14\left(\mathrm{t},{ }^{3} J_{\mathrm{C}-\mathrm{F}}=9.7 \mathrm{~Hz}\right), 135.49\left(\mathrm{t},{ }^{2} J_{\mathrm{C}-\mathrm{F}}=\right.$ $21.7 \mathrm{~Hz}), 130.61,128.94,128.59,126.98,118.48\left(\mathrm{qt}, \mathrm{CF}_{2} C F_{3},{ }^{1} J_{\mathrm{C}-\mathrm{F}}=287.3,36.8 \mathrm{~Hz}\right), 111.92$ (tq, $\left.\mathrm{CF}_{2} \mathrm{CF}_{3},{ }^{1} J_{\mathrm{C}-\mathrm{F}}=259.6,38.9 \mathrm{~Hz}\right)$.

${ }^{19}$ F NMR (470 MHz, $\left.\mathrm{CDCl}_{3}\right): \delta-81.92(\mathrm{~s}, 3 \mathrm{~F}),-115.48(\mathrm{~s}, 2 \mathrm{~F})$.

IR: $v 3020,2917,2848,1215,929,752,669,650,620 \mathrm{~cm}^{-1}$.

HR-MS (ESI): $\mathrm{m} / \mathrm{z}$ calcd. for $\left[\mathrm{C}_{10} \mathrm{H}_{6} \mathrm{~F}_{5} \mathrm{NO}_{2}+\mathrm{Na}\right]^{+}:$290.0211; found, 290.0213 . 
3.3. NMR spectra of nitroolefins

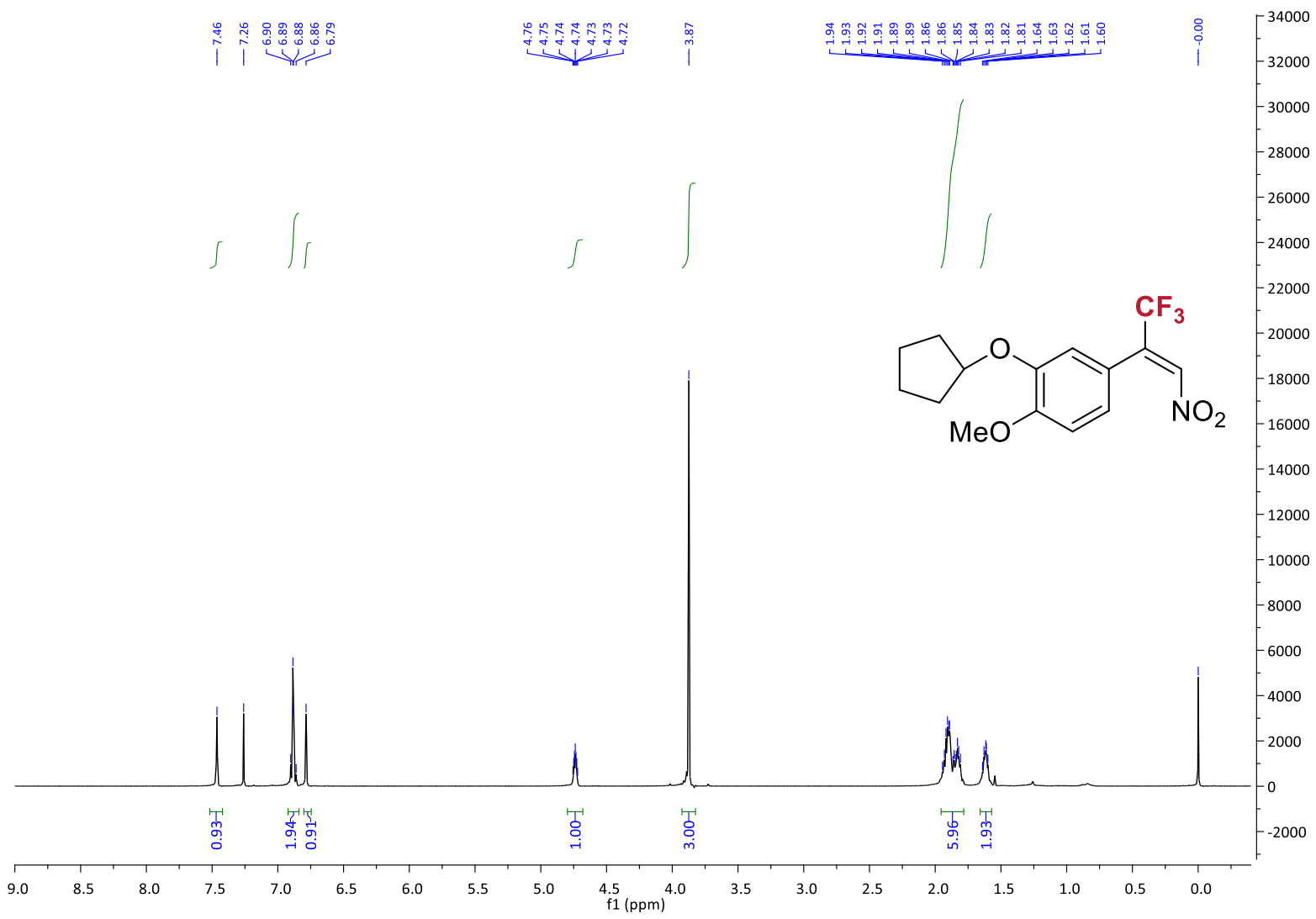

${ }^{1} \mathrm{H}$ NMR spectrum of $\mathbf{1 0}$

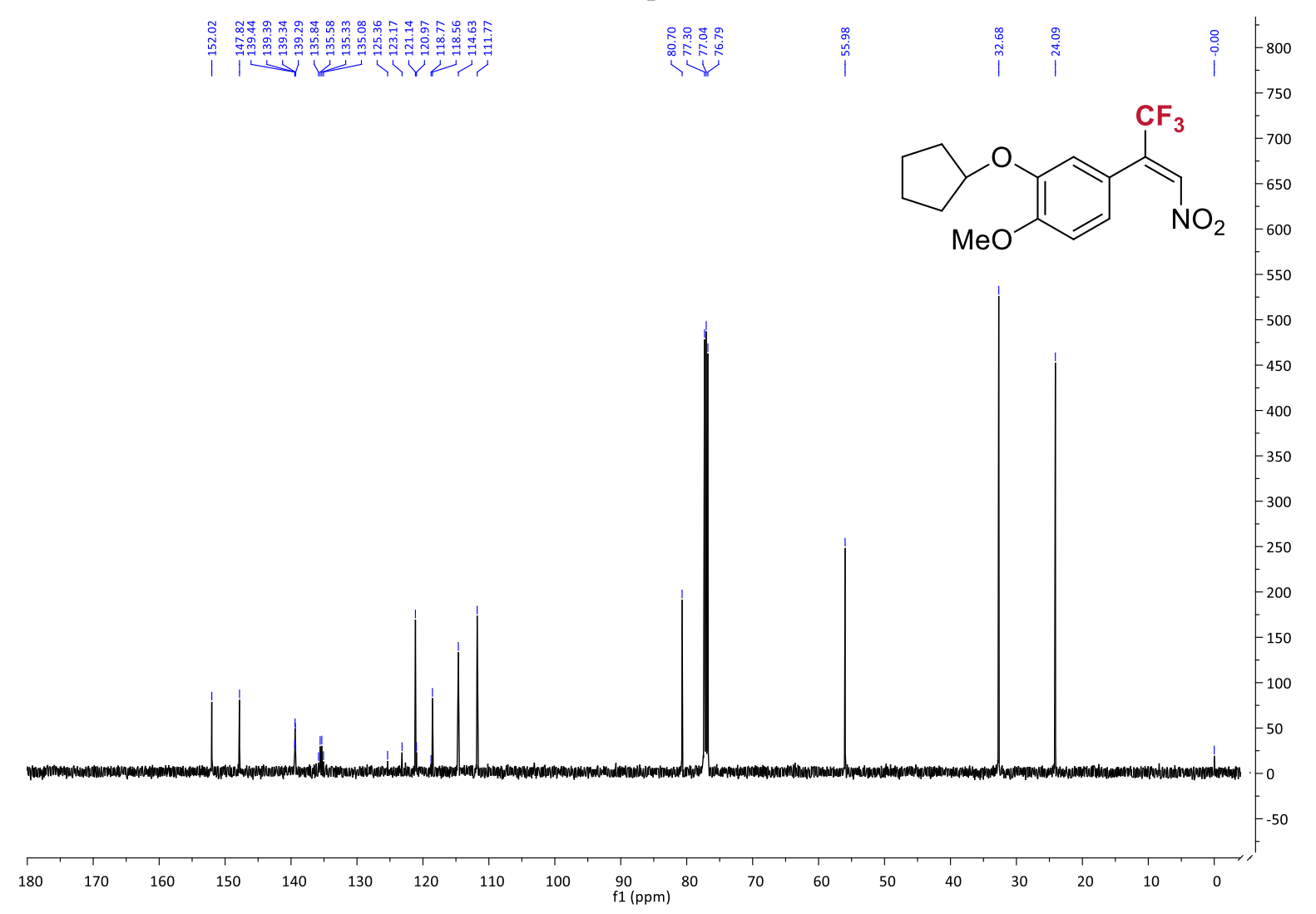

${ }^{13} \mathrm{C}$ NMR spectrum of $\mathbf{1 0}$ 


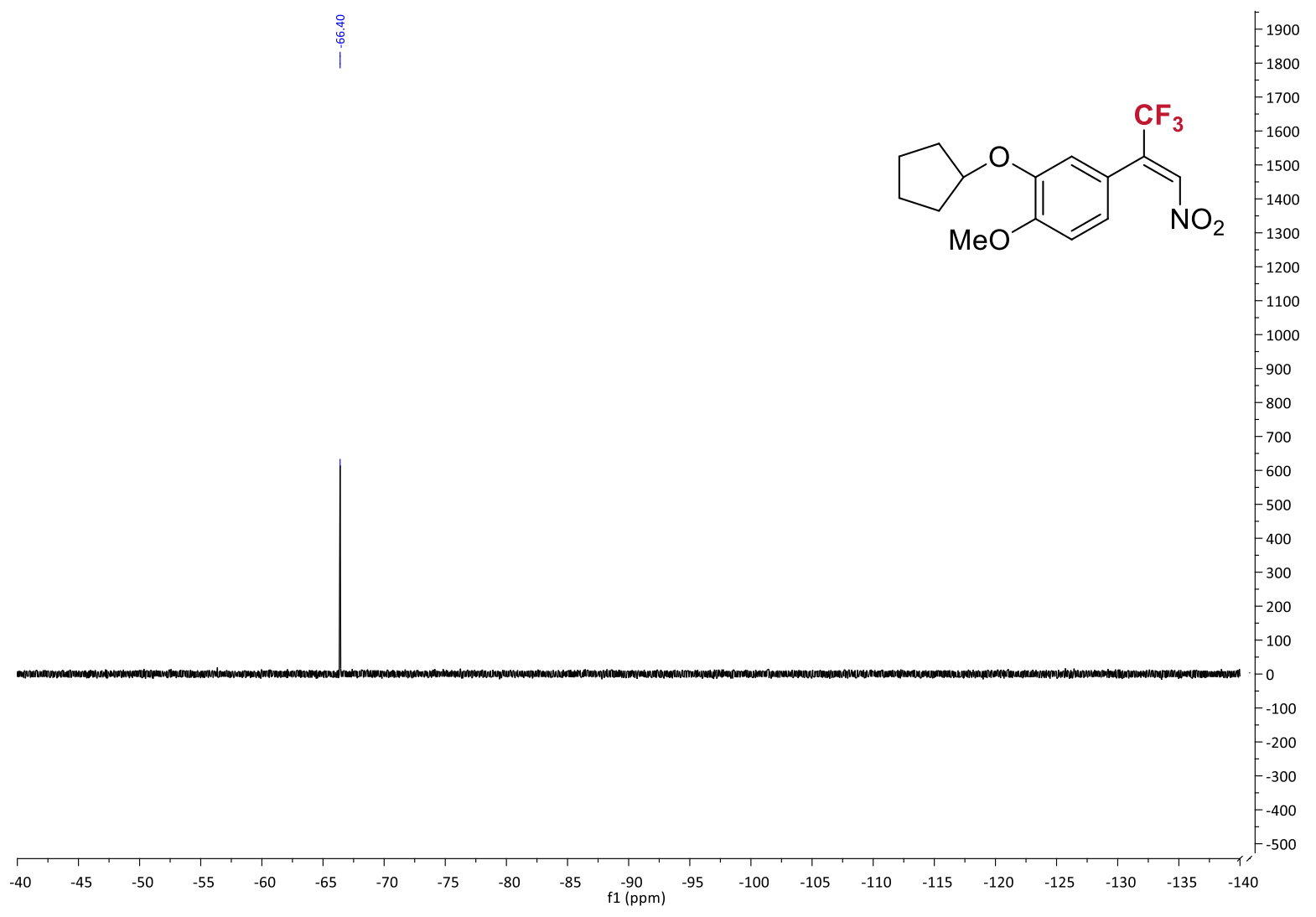

${ }^{19} \mathrm{~F}$ NMR spectrum of $\mathbf{1 0}$

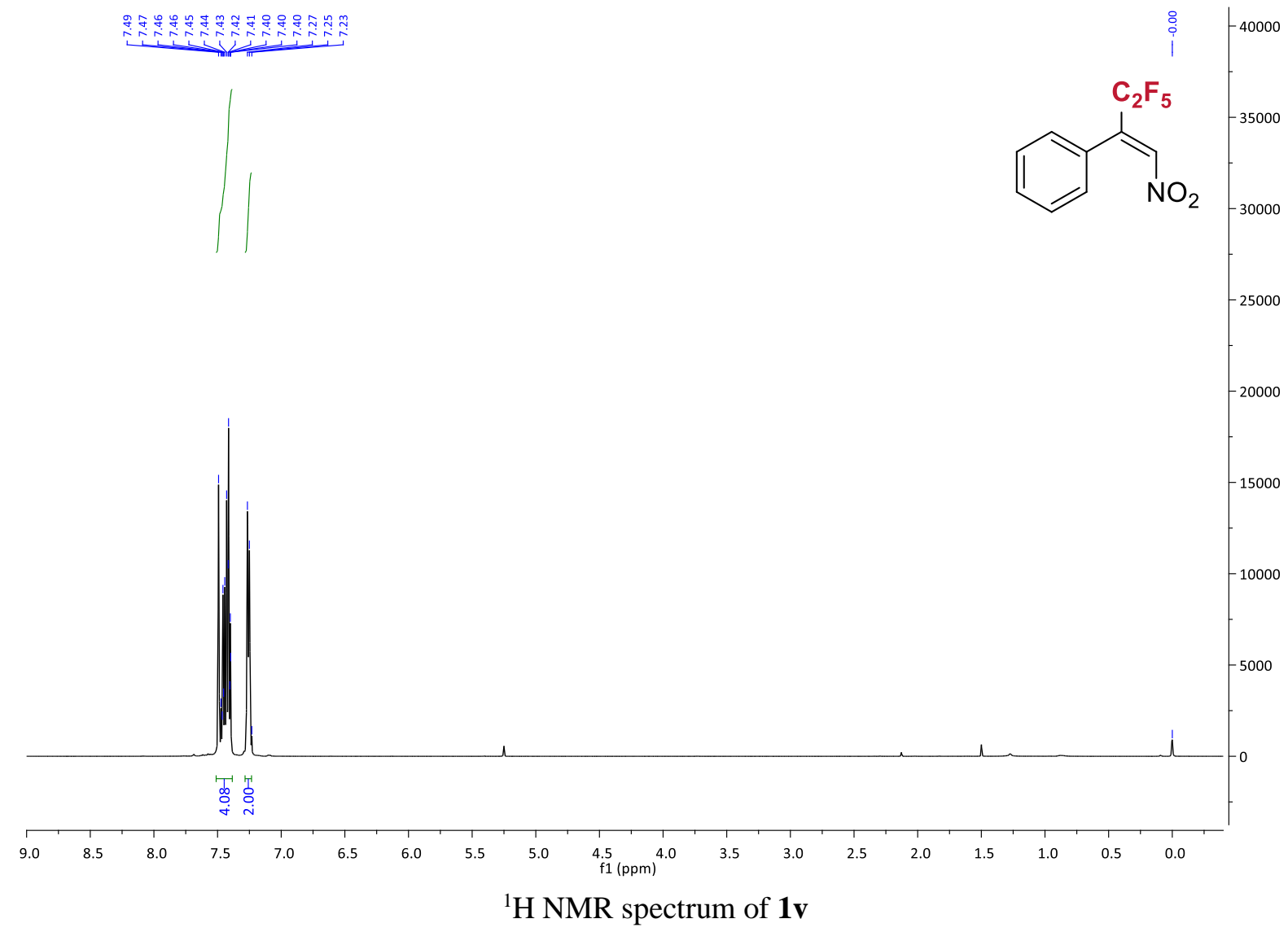




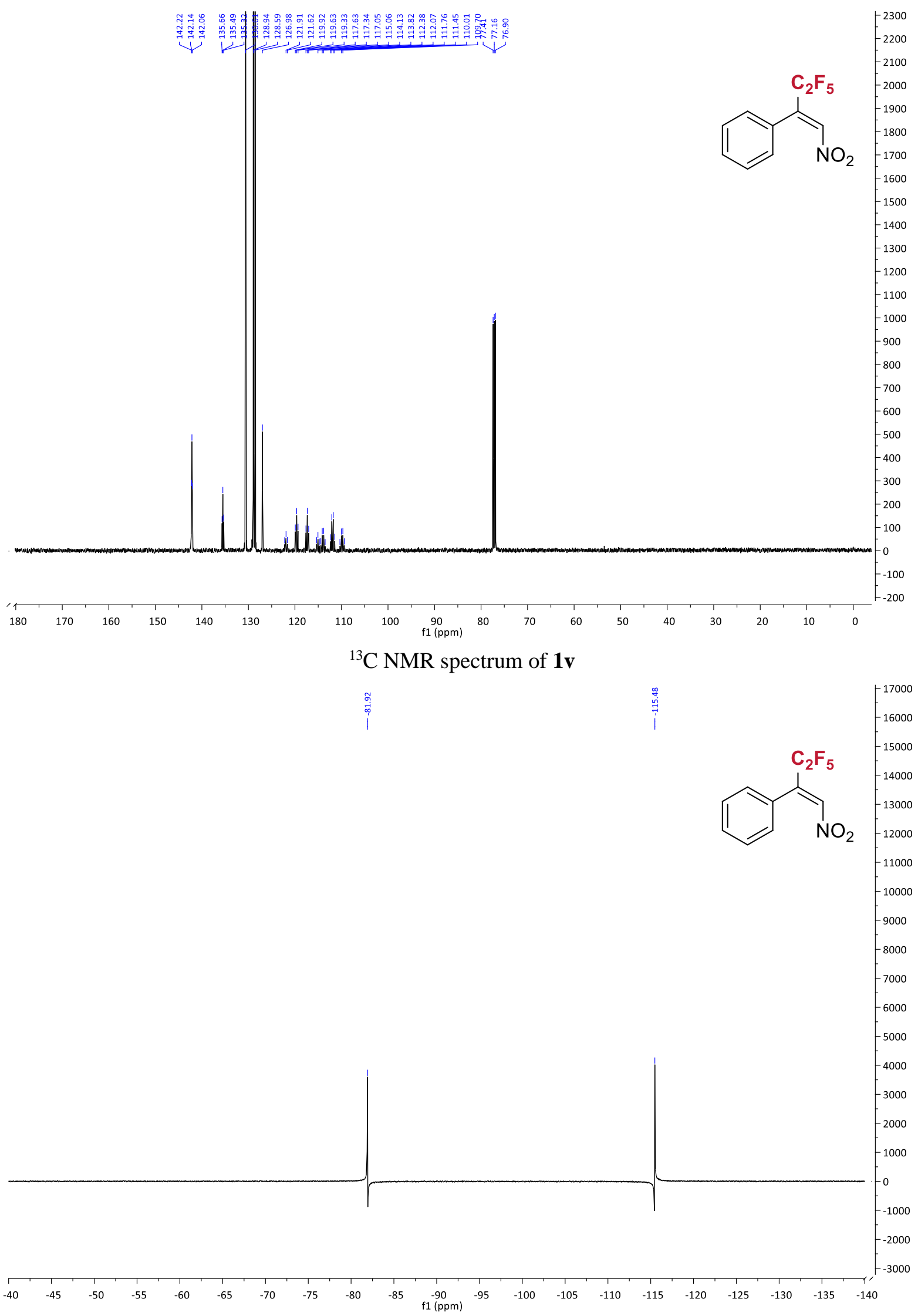

${ }^{19} \mathrm{~F}$ NMR spectrum of $\mathbf{1 v}$ 


\section{General procedure for the Michael reactions}

\subsection{General procedure for the non-enantioselective Michael reactions}

To the mixture of $\beta-\mathrm{R}_{\mathrm{F}}-\alpha$-nitroolefins (1a-w, $0.3 \mathrm{mmol}$ ), dithiomalonate (2b, 1.4 equiv., $0.42 \mathrm{mmol}$ ), toluene (HPLC grade, 5 equiv., $1.5 \mathrm{mmol}$ ), and brine (sat., $4 \mathrm{~mL}$ ) was added $\mathrm{Bu}_{3} \mathrm{~N}$ (0.3 equiv., 0.09 $\mathrm{mmol})$. The reaction mixture was stirred vigorously at $0{ }^{\circ} \mathrm{C}$ for $96 \mathrm{~h}$, after then diluted with EtOAc (3 $\mathrm{mL})$ and washed with $1 \mathrm{~N} \mathrm{HCl}(1 \mathrm{~mL})$ successively. The organic layer was extracted $(3 \mathrm{X} 3 \mathrm{~mL})$, and dried over anhydrous $\mathrm{Na}_{2} \mathrm{SO}_{4}$, and filtered. The filtrate was concentrated in vacuo, and the residue was purified by column chromatography on silica gel (acetone:hexanes $=1: 20$ to 1:10) to afford corresponding Michael addition product (3a-w).

\subsection{General procedure for the enantioselective Michael reactions}

To the mixture of $\beta-\mathrm{R}_{\mathrm{F}-} \alpha$-nitroolefins (1a-w, $\left.0.3 \mathrm{mmol}\right), \mathbf{4 f}(15 \mathrm{~mol} \%, 0.045 \mathrm{mmol})$, toluene (HPLC grade, 5 equiv., $1.5 \mathrm{mmol}$ ), and brine (sat., $4 \mathrm{~mL}$ ) was added dithiomalonate (2b, 2 equiv., $0.6 \mathrm{mmol}$ ). The reaction mixture was stirred vigorously at $0{ }^{\circ} \mathrm{C}$ for $96 \mathrm{~h}$, after then diluted with EtOAc $(3 \mathrm{~mL})$ and washed with $1 \mathrm{~N} \mathrm{HCl}(1 \mathrm{~mL})$ successively. The organic layer was extracted $(3 \mathrm{X} 3 \mathrm{~mL})$, and dried over anhydrous $\mathrm{Na}_{2} \mathrm{SO}_{4}$, and filtered. The filtrate was concentrated in vacuo, and the residue was purified by column chromatography on silica gel (acetone:hexanes $=1: 20$ to $1: 10$ ) to afford corresponding Michael addition product (3a-w).

\section{* Typical scale-up example (on 2 mmol scale)}

To the mixture of $\beta-\mathrm{R}_{\mathrm{F}}-\alpha$-nitroolefins (1f, $\left.2 \mathrm{mmol}\right), \mathbf{4 f}(15 \mathrm{~mol} \%, 0.3 \mathrm{mmol})$, toluene (HPLC grade, 5 equiv., $10 \mathrm{mmol}$ ), and brine (sat., $26 \mathrm{~mL}$ ) was added dithiomalonate (2b, 2 equiv., $4 \mathrm{mmol})$. The reaction mixture was stirred vigorously at $0{ }^{\circ} \mathrm{C}$ for $96 \mathrm{~h}$, after then diluted with EtOAc $(10 \mathrm{~mL})$ and washed with $1 \mathrm{~N} \mathrm{HCl}(10 \mathrm{~mL})$ successively. The organic layer was extracted $(3 \times 20 \mathrm{~mL})$, and dried over anhydrous $\mathrm{Na}_{2} \mathrm{SO}_{4}$, and filtered. The filtrate was concentrated in vacuo, and the residue was purified by column chromatography on silica gel (acetone:hexanes $=1: 20$ to $1: 10$ ) to afford desired Michael addition product (3f, $1.0145 \mathrm{~g}, 95 \%, 95 \%$ ee). 


\section{Synthesis of $\beta-\mathrm{CF}_{3}-\mathrm{GABA}$ analogues}

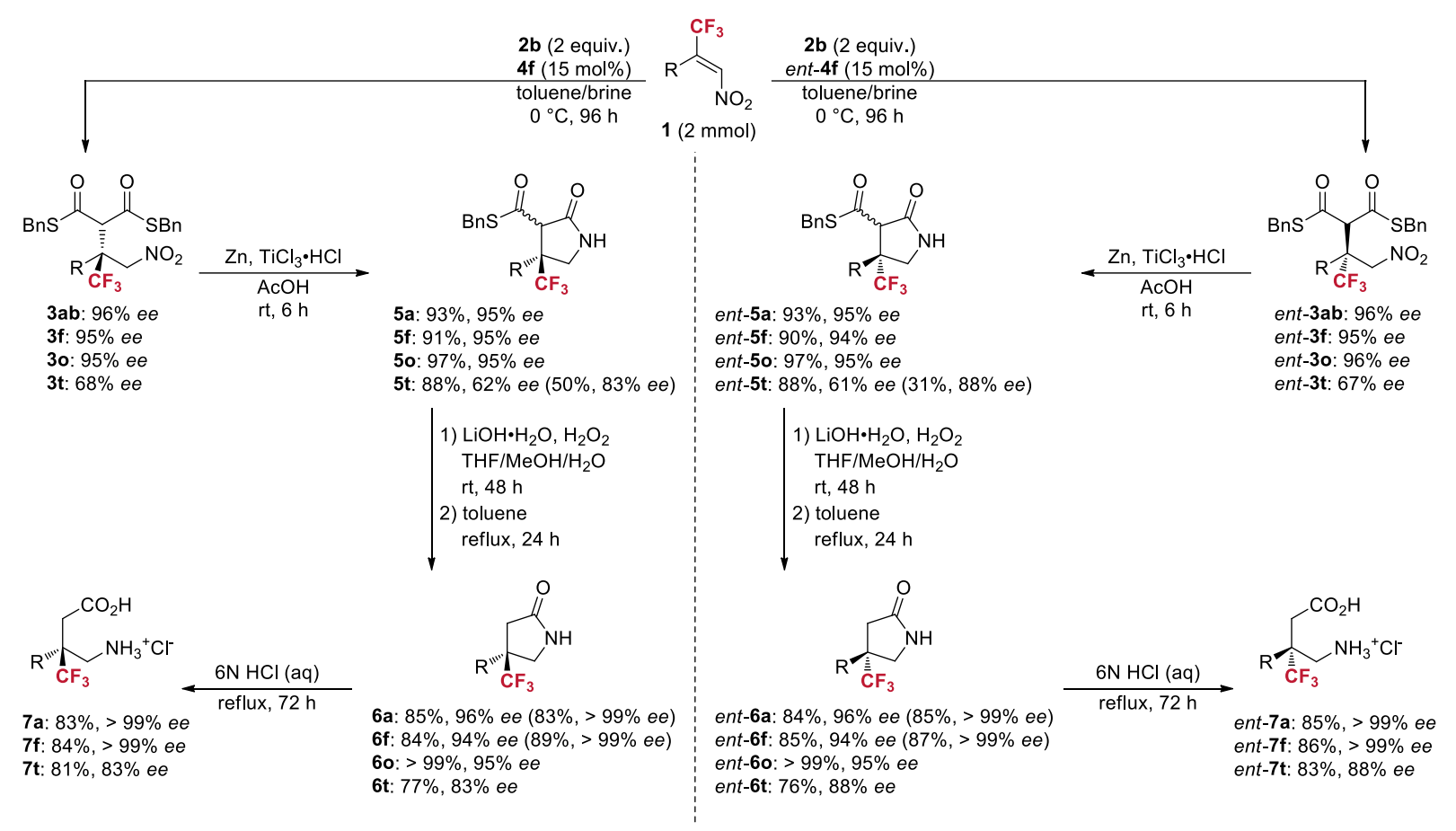

To the mixture of Michael addition product $(3,2 \mathrm{mmol})$ in $\mathrm{AcOH}(0.05 \mathrm{M}, 40 \mathrm{~mL})$ was added $\mathrm{Zn}$ powder (10 equiv., $20 \mathrm{mmol}$ ). The reaction mixture was stirred at $\mathrm{rt}$ for $4 \mathrm{~h}$. Subsequently, titanium(III) chloride solution ( $\geq 12 \% \mathrm{TiCl}_{3}$ basis, $12 \%$ in hydrochloric acid, 0.1 equiv., $0.2 \mathrm{mmol}$ ) was added to the reaction mixture dropwise, and then stirred at $\mathrm{rt}$ for $2 \mathrm{~h}$. The resulting mixture was filtered through a Celite, and washed with EtOAc. The filtrate was concentrated in vacuo, and the residue was purified by column chromatography on silica gel (EtOAc:hexanes $=1: 50$ to 2:1) to afford corresponding $\gamma$ lactam thioester (5). (5t and ent-5t were recrystallized from diethyl ether. The enantioenriched product was provided from filtrate)

The $\gamma$-lactam thioester (5) was dissolved in mixture of $\mathrm{H}_{2} \mathrm{O} / \mathrm{MeOH} / \mathrm{THF}$ (HPLC grade, $0.04 \mathrm{M}$, $\mathrm{H}_{2} \mathrm{O} / \mathrm{MeOH} / \mathrm{THF}=1: 1: 3.3 \mathrm{v} / \mathrm{v}$ ), and cooled down to $0{ }^{\circ} \mathrm{C}$. Subsequently, $\mathrm{LiOH} \cdot \mathrm{H}_{2} \mathrm{O}$ (2.6 equiv.), and $\mathrm{H}_{2} \mathrm{O}_{2}$ (34 wt. \%, 26 equiv.) were added to the reaction mixture slowly, and then stirred at rt until completion of the reaction (ca. $48-72 \mathrm{~h}$ ). The reaction mixture was quenched with sat. $\mathrm{Na}_{2} \mathrm{SO}_{3}$ at $0{ }^{\circ} \mathrm{C}$, and then stirred at $\mathrm{rt}$ for $0.5 \mathrm{~h}$. After acidification with $1 \mathrm{~N} \mathrm{HCl}$, the resulting mixture was extracted with EtOAc, and the combined organic layer was dried over anhydrous $\mathrm{Na}_{2} \mathrm{SO}_{4}$, and filtered. The filtrate was concentrated in vacuo, and the residue was used for next step without purification. The crude product, obtained from previous step, was dissolved in toluene (HPLC grade, $0.05 \mathrm{M}$ ), and stirred under reflux (ca. $110^{\circ} \mathrm{C}$ ) until completion of the reaction (ca. $24-48 \mathrm{~h}$ ). The resulting mixture was concentrated in vacuo, and the residue was purified by column chromatography on silica gel $\left(\mathrm{CH}_{2} \mathrm{Cl}_{2}: \mathrm{MeOH}=1: 50\right.$ to $\left.1: 10\right)$ to afford corresponding $\gamma$-lactam $(\mathbf{6}) .(\mathbf{6 a}$, ent-6a, $\mathbf{6 f}$, and ent-6f were recrystallized from methyl tert-butyl ether)

The $\gamma$-lactam $\left(6,0.1 \mathrm{mmol}\right.$ ) was refluxed (ca. $100-110{ }^{\circ} \mathrm{C}$ ) in $6 \mathrm{~N} \mathrm{HCl}$ (aq., $0.05 \mathrm{M}, 2 \mathrm{~mL}$ ) until completion of the hydrolysis (ca. $72-96 \mathrm{~h}$ ). After cooling, the reaction mixture was washed with $\mathrm{Et}_{2} \mathrm{O}$. The volatile components were removed in vacuo, to afford corresponding $\mathrm{HCl}$ salt of $\gamma$-amino acid (7). 


\section{Analytical data of the products $(3-7)$}<smiles>CC(c1ccccc1)(C(C(=O)Cc1ccccc1)C(=O)[Sn]c1ccccc1)C(F)(F)F</smiles>

3ab, New Compound, (yellow solid, 93\% yield, $148 \mathrm{mg}$ ).

$\mathbf{R}_{\mathbf{f}}=0.15$ (acetone:hexanes, $1: 10 \mathrm{v} / \mathrm{v}$ ).

mp: $89^{\circ} \mathrm{C}$.

$[\alpha]_{\mathrm{D}}{ }^{20}=-27.2\left(\mathrm{c}=1.0, \mathrm{CHCl}_{3}, 96 \% e e\right)$.

${ }^{1} \mathbf{H}$ NMR $\left(500 \mathrm{MHz}, \mathrm{CDCl}_{3}, \mathrm{Me}_{4} \mathrm{Si}\right): \delta 7.37(\mathrm{~m}, 2 \mathrm{H}), 7.31-7.18(\mathrm{~m}, 11 \mathrm{H}), 6.96$ $(\mathrm{m}, 2 \mathrm{H}), 6.17\left(\mathrm{~d}, J=15.2 \mathrm{~Hz}, 1 \mathrm{H}\right.$, diastereotopic proton A, $\left.\mathrm{CH}_{2} \mathrm{NO}_{2}\right), 5.93(\mathrm{~d}, J=$ $15.2 \mathrm{~Hz}, 1 \mathrm{H}$, diastereotopic proton $\left.\mathrm{B}, \mathrm{CH}_{2} \mathrm{NO}_{2}\right), 4.44(\mathrm{~s}, 1 \mathrm{H}), 4.22(\mathrm{~d}, J=13.8 \mathrm{~Hz}, 1 \mathrm{H}$, diastereotopic proton $\left.\mathrm{A}, \mathrm{SCH}_{2} \mathrm{Ph}\right), 4.13\left(\mathrm{~d}, J=13.8 \mathrm{~Hz}, 1 \mathrm{H}\right.$, diastereotopic proton $\left.\mathrm{B}, \mathrm{SCH}_{2} \mathrm{Ph}\right), 3.93(\mathrm{~d}, J=13.9 \mathrm{~Hz}$, $1 \mathrm{H}$, diastereotopic proton $\left.\mathrm{C}, \mathrm{SCH}_{2} \mathrm{Ph}\right), 3.76\left(\mathrm{~d}, J=13.9 \mathrm{~Hz}, 1 \mathrm{H}\right.$, diastereotopic proton $\left.\mathrm{D}, \mathrm{SCH}_{2} \mathrm{Ph}\right)$.

${ }^{13} \mathbf{C}$ NMR $\left(126 \mathrm{MHz}, \mathrm{CDCl}_{3}, \mathrm{Me}_{4} \mathrm{Si}\right): \delta 189.23,188.86,135.58,135.43,130.83,129.00,128.98$, $128.79,128.76,128.58,127.75,127.60,127.01,125.35\left(\mathrm{q},{ }^{1} J_{\mathrm{C}-\mathrm{F}}=287.9 \mathrm{~Hz}\right), 73.06,70.88,57.01(\mathrm{q}$, $\left.{ }^{2} J_{\mathrm{C}-\mathrm{F}}=25.5 \mathrm{~Hz}\right), 34.69$.

${ }^{19}$ F NMR $\left(470 \mathrm{MHz}, \mathrm{CDCl}_{3}\right): \delta-61.12(\mathrm{~s}, 3 \mathrm{~F})$.

IR: $v$ 3065, 3031, 2928, 1693, 1561, 1454, 1373, 1223, 1184, 1152, $975 \mathrm{~cm}^{-1}$.

HR-MS (ESI): $\mathrm{m} / \mathrm{z}$ calcd. for $\left[\mathrm{C}_{26} \mathrm{H}_{22} \mathrm{~F}_{3} \mathrm{NO}_{4} \mathrm{~S}_{2}+\mathrm{Na}\right]^{+}$: 556.0835 ; found, 556.0839.

Enantiomeric excess: $\left(\right.$ CHIRALPAK $^{\circledR} \mathrm{IB}$; Hexane: ${ }^{i} \mathrm{PrOH}=90: 10$; flow rate: $1.0 \mathrm{~mL} / \mathrm{min}$;; $254 \mathrm{~nm}$ ): $\mathrm{t}_{\mathrm{S}}=10.12$ min.(minor), $\mathrm{t}_{\mathrm{R}}=12.71$ min.(major); $96 \%$ ee.<smiles>O=C(Cc1ccccc1)C(C(=O)[Sn]Br)C(C[N+](=O)[O-])(c1ccccc1)c1ccccc1</smiles>

ent-3ab, New Compound, (yellow solid, $92 \%$ yield, $147 \mathrm{mg}$ ).

$\mathbf{R}_{\mathbf{f}}=0.15$ (acetone:hexanes, $1: 10 \mathrm{v} / \mathrm{v}$ ).

Enantiomeric excess: $\left(\right.$ CHIRALPAK $^{\circledR} \mathrm{IB}$; Hexane: ${ }^{i} \mathrm{PrOH}=90: 10$; flow rate: 1.0 $\mathrm{mL} / \min$.; $254 \mathrm{~nm}$ ): $\mathrm{t}_{\mathrm{S}}=10.66 \min$.(major), $\mathrm{t}_{\mathrm{R}}=13.66 \mathrm{~min}$.(minor); $96 \%$ ee.<smiles>CC(C[N+](=O)[O-])(c1ccc2ccccc2c1)C(C(=O)Br)C(=O)Cc1ccccc1</smiles>

3b, New Compound, (white oil, 92\% yield, $161 \mathrm{mg}$ ).

$\mathbf{R}_{\mathbf{f}}=0.13$ (acetone:hexanes, $1: 10 \mathrm{v} / \mathrm{v}$ ).

$[\boldsymbol{\alpha}]_{\mathbf{D}}{ }^{20}=-14.6\left(\mathrm{c}=1.0, \mathrm{CHCl}_{3}, 94 \% e e\right)$.

${ }^{1} \mathbf{H}$ NMR $\left(500 \mathrm{MHz}, \mathrm{CDCl}_{3}, \mathrm{Me}_{4} \mathrm{Si}\right): \delta 7.94(\mathrm{~s}, 1 \mathrm{H}), 7.75(\mathrm{dd}, J=14.9,8.0$ $\mathrm{Hz}, 2 \mathrm{H}), 7.65(\mathrm{~d}, J=8.9 \mathrm{~Hz}, 1 \mathrm{H}), 7.53-7.42(\mathrm{~m}, 3 \mathrm{H}), 7.29-7.20(\mathrm{~m}, 5 \mathrm{H})$, $7.07(\mathrm{t}, J=7.4 \mathrm{~Hz}, 1 \mathrm{H}), 6.95(\mathrm{t}, J=7.6 \mathrm{~Hz}, 2 \mathrm{H}), 6.62(\mathrm{~d}, J=7.6 \mathrm{~Hz}, 2 \mathrm{H})$, $6.33\left(\mathrm{~d}, J=15.3 \mathrm{~Hz}, 1 \mathrm{H}\right.$, diastereotopic proton $\left.\mathrm{A}, \mathrm{CH}_{2} \mathrm{NO}_{2}\right), 6.04(\mathrm{~d}, J=15.3 \mathrm{~Hz}, 1 \mathrm{H}$, diastereotopic proton $\left.\mathrm{B}, \mathrm{CH}_{2} \mathrm{NO}_{2}\right), 4.58(\mathrm{~s}, 1 \mathrm{H}), 4.22\left(\mathrm{~d}, J=13.8 \mathrm{~Hz}, 1 \mathrm{H}\right.$, diastereotopic proton $\left.\mathrm{A}, \mathrm{SCH} \mathrm{H}_{2} \mathrm{Ph}\right), 4.13(\mathrm{~d}$, $J=13.8 \mathrm{~Hz}, 1 \mathrm{H}$, diastereotopic proton $\left.\mathrm{B}, \mathrm{SCH}_{2} \mathrm{Ph}\right), 3.80(\mathrm{~d}, J=13.9 \mathrm{~Hz}, 1 \mathrm{H}$, diastereotopic proton $\mathrm{C}$, $\left.\mathrm{SCH}_{2} \mathrm{Ph}\right), 3.65\left(\mathrm{~d}, J=13.9 \mathrm{~Hz}, 1 \mathrm{H}\right.$, diastereotopic proton $\left.\mathrm{D}, \mathrm{SCH}_{2} \mathrm{Ph}\right)$.

${ }^{13} \mathrm{C}$ NMR $\left(126 \mathrm{MHz}, \mathrm{CDCl}_{3}, \mathrm{Me}_{4} \mathrm{Si}\right): \delta 189.24,188.86,135.58,134.94,132.79,128.90,128.78$, $128.70,128.44,128.40,128.23,127.75,127.42,127.40,127.37,127.27,126.85,125.47\left(\mathrm{q},{ }^{1} J_{\mathrm{C}-\mathrm{F}}=\right.$ $288.0 \mathrm{~Hz}), 123.77,73.26,70.78,57.24\left(\mathrm{q},{ }^{2} J_{\mathrm{C}-\mathrm{F}}=25.5 \mathrm{~Hz}\right), 34.73,34.64$.

${ }^{19}$ F NMR $\left(470 \mathrm{MHz}, \mathrm{CDCl}_{3}\right): \delta-60.86(\mathrm{~s}, 3 \mathrm{~F})$.

IR: $v$ 3063, 3032, 2926, 1694, 1561, 1496, 1454, 1372, 1187, $978 \mathrm{~cm}^{-1}$.

HR-MS (ESI): $\mathrm{m} / \mathrm{z}$ calcd. for $\left[\mathrm{C}_{30} \mathrm{H}_{24} \mathrm{~F}_{3} \mathrm{NO}_{4} \mathrm{~S}_{2}+\mathrm{Na}\right]^{+}$: 606.0991 ; found, 606.0994 .

Enantiomeric excess: $\left(\right.$ CHIRALPAK $^{\circledR} \mathrm{IB}$; Hexane: ${ }^{i} \mathrm{PrOH}=90: 10$; flow rate: $1.0 \mathrm{~mL} / \mathrm{min}$;; $254 \mathrm{~nm}$ ): $\mathrm{t}_{\mathrm{S}}=9.76 \mathrm{~min}$.(minor), $\mathrm{t}_{\mathrm{R}}=12.16 \mathrm{~min}$.(major); $94 \%$ ee . 
<smiles>O=C(Br)C(C(=O)Br)C(C[N+](=O)[O-])(c1ccccc1)C(F)(F)c1ccccc1</smiles>

3c, New Compound, (yellow solid, $21 \%$ yield, $30 \mathrm{mg}$ ).

$\mathbf{R}_{\mathbf{f}}=0.06$ (acetone:hexanes, $1: 10 \mathrm{v} / \mathrm{v}$ ).

mp: $99{ }^{\circ} \mathrm{C}$.

$[\boldsymbol{\alpha}]_{\mathbf{D}}{ }^{20}=-57.2\left(\mathrm{c}=0.5, \mathrm{CHCl}_{3}, 95 \% e e\right)$.

${ }^{1} \mathbf{H}$ NMR $\left(500 \mathrm{MHz}, \mathrm{CDCl}_{3}, \mathrm{Me}_{4} \mathrm{Si}\right): \delta 7.33-7.18(\mathrm{~m}, 10 \mathrm{H}), 7.12(\mathrm{t}, J=7.8 \mathrm{~Hz}$, $1 \mathrm{H}), 7.02-6.91(\mathrm{~m}, 4 \mathrm{H}), 6.12(\mathrm{~d}, J=17.3 \mathrm{~Hz}, 1 \mathrm{H}$, diastereotopic proton A, $\left.\mathrm{CH}_{2} \mathrm{NO}_{2}\right), 6.07\left(\mathrm{~d}, J=17.2 \mathrm{~Hz}, 1 \mathrm{H}\right.$, diastereotopic proton $\left.\mathrm{B}, \mathrm{CH}_{2} \mathrm{NO}_{2}\right), 4.67(\mathrm{~s}, 1 \mathrm{H}), 4.22(\mathrm{~d}, J=13.8$ $\mathrm{Hz}, 1 \mathrm{H}$, diastereotopic proton $\left.\mathrm{A}, \mathrm{SCH} \mathrm{S}_{2} \mathrm{Ph}\right), 4.15\left(\mathrm{~d}, J=13.8 \mathrm{~Hz}, 1 \mathrm{H}\right.$, diastereotopic proton $\left.\mathrm{B}, \mathrm{SCH}_{2} \mathrm{Ph}\right)$, $3.96\left(\mathrm{~d}, J=14.0 \mathrm{~Hz}, 1 \mathrm{H}\right.$, diastereotopic proton $\left.\mathrm{C}, \mathrm{SCH}_{2} \mathrm{Ph}\right), 3.79(\mathrm{~d}, J=13.9 \mathrm{~Hz}, 1 \mathrm{H}$, diastereotopic proton $\left.\mathrm{D}, \mathrm{SCH} \mathrm{H}_{2} \mathrm{Ph}\right)$.

${ }^{13} \mathrm{C} \mathrm{NMR}\left(126 \mathrm{MHz}, \mathrm{CDCl}_{3}, \mathrm{Me}_{4} \mathrm{Si}\right): \delta 189.33,189.20,160.43\left(\mathrm{~d},{ }^{1} J_{\mathrm{C}-\mathrm{F}}=248.8 \mathrm{~Hz}\right), 135.57\left(\mathrm{~d},{ }^{3} J_{\mathrm{C}-\mathrm{F}}=\right.$ $6.7 \mathrm{~Hz}), 131.22\left(\mathrm{~d},{ }^{3} J_{\mathrm{C}-\mathrm{F}}=9.6 \mathrm{~Hz}\right), 128.81,128.76,128.58,128.02,127.69\left(\mathrm{~d},{ }^{2} J_{\mathrm{C}-\mathrm{F}}=20.5 \mathrm{~Hz}\right), 124.90$ $\left(\mathrm{q},{ }^{1} J_{\mathrm{C}-\mathrm{F}}=288.4 \mathrm{~Hz}\right), 124.79\left(\mathrm{~d},{ }^{4} J_{\mathrm{C}-\mathrm{F}}=3.1 \mathrm{~Hz}\right), 117.24\left(\mathrm{~d},{ }^{2} J_{\mathrm{C}-\mathrm{F}}=24.7 \mathrm{~Hz}\right), 72.72,68.67,55.35(\mathrm{qd}$, $\left.{ }^{2} J_{\mathrm{C}-\mathrm{F}}=27.5,2.9 \mathrm{~Hz}\right), 34.72,34.60$.

${ }^{19}$ F NMR $\left(470 \mathrm{MHz}, \mathrm{CDCl}_{3}\right): \delta-59.85(\mathrm{~d}, J=14.9 \mathrm{~Hz}, 3 \mathrm{~F}),-107.97(\mathrm{q}, J=15.8 \mathrm{~Hz}, 1 \mathrm{~F})$.

IR: $v$ 3065, 3031, 2928, 1696, 1564, 1218, 972, 756, $700 \mathrm{~cm}^{-1}$.

HR-MS (ESI): $\mathrm{m} / \mathrm{z}$ calcd. for $\left[\mathrm{C}_{26} \mathrm{H}_{21} \mathrm{~F}_{4} \mathrm{NO}_{4} \mathrm{~S}_{2}+\mathrm{Na}\right]^{+}$: 574.0740; found, 574.0743.

Enantiomeric excess: $\left(\mathrm{CHIRALPAK}^{\circledR} \mathrm{IB}\right.$; Hexane: ${ }^{i} \mathrm{PrOH}=90: 10$; flow rate: $1.0 \mathrm{~mL} / \mathrm{min}$.; $254 \mathrm{~nm}$ ): $\mathrm{t}_{\mathrm{S}}=10.12$ min.(minor), $\mathrm{t}_{\mathrm{R}}=11.70$ min.(major); $95 \%$ ee.<smiles>O=C(Br)C(C(=O)CCc1ccccc1)C(C[N+](=O)[O-])(c1ccccc1)c1ccccc1</smiles>
ent-3c, New Compound, (yellow solid, $24 \%$ yield, $40 \mathrm{mg}$ ). $\mathbf{R}_{\mathbf{f}}=0.06$ (acetone:hexanes, $\left.1: 10 \mathrm{v} / \mathrm{v}\right)$.

Enantiomeric excess: $\left(\right.$ CHIRALPAK $^{\circledR}$ IB; Hexane: ${ }^{i} \mathrm{PrOH}=90: 10$; flow rate: 1.0 $\mathrm{mL} / \min$.; $254 \mathrm{~nm}$ ): $\mathrm{t}_{\mathrm{S}}=10.06$ min.(major), $\mathrm{t}_{\mathrm{R}}=11.73$ min.(minor); $95 \%$ ee.<smiles>CC(C[N+](=O)[O-])(c1cccc(F)c1)[C@@H](C(=O)Br)C(=O)Sc1ccccc1</smiles>

3d, New Compound, (yellow solid, 90\% yield, $148 \mathrm{mg}$ ).

$\mathbf{R}_{\mathbf{f}}=0.14($ acetone:hexanes, $1: 10 \mathrm{v} / \mathrm{v})$.

mp: $81^{\circ} \mathrm{C}$.

$[\alpha]_{\mathrm{D}}{ }^{20}=-16.1\left(\mathrm{c}=1.0, \mathrm{CHCl}_{3}, 94 \% e e\right)$.

${ }^{1} \mathbf{H}$ NMR $\left(500 \mathrm{MHz}, \mathrm{CDCl}_{3}, \mathrm{Me}_{4} \mathrm{Si}\right): \delta 7.32-7.25(\mathrm{~m}, 3 \mathrm{H}), 7.23(\mathrm{~m}, 5 \mathrm{H})$, $7.19(\mathrm{~m}, 1 \mathrm{H}), 7.12(\mathrm{~m}, 2 \mathrm{H}), 7.02-6.97(\mathrm{~m}, 2 \mathrm{H}), 6.97-6.92(\mathrm{~m}, 1 \mathrm{H}), 6.11(\mathrm{~d}$, $J=15.3 \mathrm{~Hz}, 1 \mathrm{H}$, diastereotopic proton A, $\left.\mathrm{CH}_{2} \mathrm{NO}_{2}\right), 5.93(\mathrm{~d}, J=15.3 \mathrm{~Hz}, 1 \mathrm{H}$, diastereotopic proton $\mathrm{B}$, $\left.\mathrm{CH}_{2} \mathrm{NO}_{2}\right), 4.40(\mathrm{~s}, 1 \mathrm{H}), 4.23\left(\mathrm{~d}, J=13.8 \mathrm{~Hz}, 1 \mathrm{H}\right.$, diastereotopic proton $\left.\mathrm{A}, \mathrm{SCH}_{2} \mathrm{Ph}\right), 4.14(\mathrm{~d}, J=13.9$ $\mathrm{Hz}, 1 \mathrm{H}$, diastereotopic proton $\left.\mathrm{B}, \mathrm{SCH} \mathrm{H}_{2} \mathrm{Ph}\right), 3.98\left(\mathrm{~d}, J=13.9 \mathrm{~Hz}, 1 \mathrm{H}\right.$, diastereotopic proton $\left.\mathrm{C}, \mathrm{SCH}_{2} \mathrm{Ph}\right)$, $3.80\left(\mathrm{~d}, J=13.9 \mathrm{~Hz}, 1 \mathrm{H}\right.$, diastereotopic proton $\left.\mathrm{D}, \mathrm{SCH}_{2} \mathrm{Ph}\right)$.

${ }^{13} \mathrm{C} \mathrm{NMR}\left(126 \mathrm{MHz}, \mathrm{CDCl}_{3}, \mathrm{Me}_{4} \mathrm{Si}\right): \delta 189.05,188.62,162.68\left(\mathrm{~d},{ }^{1} J_{\mathrm{C}-\mathrm{F}}=247.4 \mathrm{~Hz}\right), 135.49,135.40$, $133.17\left(\mathrm{~d},{ }^{3} J_{\mathrm{C}-\mathrm{F}}=7.2 \mathrm{~Hz}\right), 130.50\left(\mathrm{~d},{ }^{3} J_{\mathrm{C}-\mathrm{F}}=8.3 \mathrm{~Hz}\right), 128.82,128.71,128.67,127.82,127.71,125.10$ $\left(\mathrm{q},{ }^{1} J_{\mathrm{C}-\mathrm{F}}=288.0 \mathrm{~Hz}\right), 122.96,116.20\left(\mathrm{~d},{ }^{2} J_{\mathrm{C}-\mathrm{F}}=20.8 \mathrm{~Hz}\right), 114.83\left(\mathrm{~d},{ }^{2} J_{\mathrm{C}-\mathrm{F}}=24.6 \mathrm{~Hz}\right), 73.02,70.55$, $56.83\left(\mathrm{q},{ }^{2} J_{\mathrm{C}-\mathrm{F}}=25.4 \mathrm{~Hz}\right), 34.81,34.77$.

${ }^{19}$ F NMR $\left(470 \mathrm{MHz}, \mathrm{CDCl}_{3}\right): \delta-61.54(\mathrm{~s}, 3 \mathrm{~F}),-109.94(\mathrm{~s}, 1 \mathrm{~F})$.

IR: $v$ 3066, 3033, 2929, 1695, 1562, 1496, 1453, 1373, 1189, $980 \mathrm{~cm}^{-1}$.

HR-MS (ESI): $\mathrm{m} / \mathrm{z}$ calcd. for $\left[\mathrm{C}_{26} \mathrm{H}_{21} \mathrm{~F}_{4} \mathrm{NO}_{4} \mathrm{~S}_{2}+\mathrm{Na}\right]^{+}:$574.0740; found, 574.0744.

Enantiomeric excess: $\left(\mathrm{CHIRALPAK}^{\circledR} \mathrm{IB}\right.$; Hexane: ${ }^{i} \mathrm{PrOH}=90: 10$; flow rate: $1.0 \mathrm{~mL} / \mathrm{min}$.; $254 \mathrm{~nm}$ ): $\mathrm{t}_{\mathrm{S}}=9.66 \mathrm{~min} .\left(\right.$ minor), $\mathrm{t}_{\mathrm{R}}=13.43 \mathrm{~min}$.(major); $94 \%$ ee. 


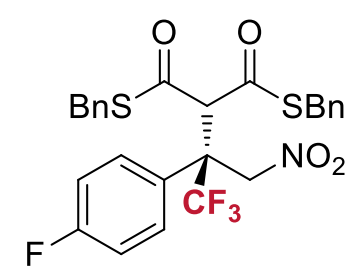

3e, New Compound, (colorless oil, 92\% yield, $152 \mathrm{mg}$ ).

$\mathbf{R}_{\mathbf{f}}=0.16$ (acetone:hexanes, $1: 10 \mathrm{v} / \mathrm{v}$ ).

$[\boldsymbol{\alpha}]_{\mathbf{D}}{ }^{20}=-2.8\left(\mathrm{c}=1.0, \mathrm{CHCl}_{3}, 95 \% e e\right)$.

${ }^{1} \mathbf{H}$ NMR $\left(500 \mathrm{MHz}, \mathrm{CDCl}_{3}, \mathrm{Me}_{4} \mathrm{Si}\right): \delta 7.37-7.21(\mathrm{~m}, 10 \mathrm{H}), 7.00(\mathrm{~m}, 2 \mathrm{H})$, $6.84(\mathrm{t}, J=8.2 \mathrm{~Hz}, 2 \mathrm{H}), 6.10(\mathrm{~d}, J=15.1 \mathrm{~Hz}, 1 \mathrm{H}$, diastereotopic proton A, $\left.\mathrm{CH}_{2} \mathrm{NO}_{2}\right), 5.90\left(\mathrm{~d}, J=15.1 \mathrm{~Hz}, 1 \mathrm{H}\right.$, diastereotopic proton $\left.\mathrm{B}, \mathrm{CH}_{2} \mathrm{NO}_{2}\right), 4.38(\mathrm{~s}$, $1 \mathrm{H}), 4.24\left(\mathrm{~d}, J=13.8 \mathrm{~Hz}, 1 \mathrm{H}\right.$, diastereotopic proton $\left.\mathrm{A}, \mathrm{SCH} \mathrm{H}_{2} \mathrm{Ph}\right), 4.14(\mathrm{~d}, J=13.8 \mathrm{~Hz}, 1 \mathrm{H}$, diastereotopic proton $\left.\mathrm{B}, \mathrm{SCH}_{2} \mathrm{Ph}\right), 3.99\left(\mathrm{~d}, J=14.0 \mathrm{~Hz}, 1 \mathrm{H}\right.$, diastereotopic proton $\left.\mathrm{C}, \mathrm{SCH} \mathrm{H}_{2} \mathrm{Ph}\right), 3.76(\mathrm{~d}$, $J=14.0 \mathrm{~Hz}, 1 \mathrm{H}$, diastereotopic proton $\left.\mathrm{D}, \mathrm{SCH}_{2} \mathrm{Ph}\right)$.

${ }^{13} \mathrm{C}$ NMR $\left(126 \mathrm{MHz}, \mathrm{CDCl}_{3}, \mathrm{Me}_{4} \mathrm{Si}\right): \delta 189.10,188.68,162.68\left(\mathrm{~d},{ }^{1} J_{\mathrm{C}-\mathrm{F}}=250.6 \mathrm{~Hz}\right), 135.54,135.50$, $129.24\left(\mathrm{~d},{ }^{3} J_{\mathrm{C}-\mathrm{F}}=8.3 \mathrm{~Hz}\right), 128.82,128.78,128.67,127.82,127.73,126.51\left(\mathrm{~d},{ }^{4} J_{\mathrm{C}-\mathrm{F}}=3.5 \mathrm{~Hz}\right), 125.23$ $\left(\mathrm{q},{ }^{1} J_{\mathrm{C}-\mathrm{F}}=287.9 \mathrm{~Hz}\right), 115.97\left(\mathrm{~d},{ }^{2} J_{\mathrm{C}-\mathrm{F}}=21.7 \mathrm{~Hz}\right), 73.15,70.78,56.60\left(\mathrm{q},{ }^{2} J_{\mathrm{C}-\mathrm{F}}=25.6 \mathrm{~Hz}\right), 34.77,34.74$. ${ }^{19}$ F NMR $\left(470 \mathrm{MHz}, \mathrm{CDCl}_{3}\right): \delta-61.77$ (s, 3F), -111.73 (s, 1F).

IR: $v$ 3065, 3031, 2356, 1695, 1562, 1518, 1220, 1184, 1084, $978 \mathrm{~cm}^{-1}$.

HR-MS (ESI): $\mathrm{m} / \mathrm{z}$ calcd. for $\left[\mathrm{C}_{26} \mathrm{H}_{21} \mathrm{~F}_{4} \mathrm{NO}_{4} \mathrm{~S}_{2}+\mathrm{Na}\right]^{+}:$574.0740; found, 574.0743.

Enantiomeric excess: $\left(\mathrm{CHIRALPAK}^{\circledR} \mathrm{IB}\right.$; Hexane: ${ }^{i} \mathrm{PrOH}=90: 10$; flow rate: $1.0 \mathrm{~mL} / \mathrm{min}$; $254 \mathrm{~nm}$ ): $\mathrm{t}_{\mathrm{S}}=21.09$ min.(minor), $\mathrm{t}_{\mathrm{R}}=31.19$ min. (major); $95 \%$ ee .<smiles>O=C(Br)C(C(=O)Br)C(C[N+](=O)[O-])(c1ccc(Cl)cc1)C(F)(F)F</smiles>

3f, New Compound, (white oil, 95\% yield, $162 \mathrm{mg}$ ).

$\mathbf{R}_{\mathbf{f}}=0.17$ (acetone:hexanes, $1: 10 \mathrm{v} / \mathrm{v}$ ).

$[\alpha]_{\mathrm{D}}{ }^{\mathbf{2 0}}=-2.2\left(\mathrm{c}=1.0, \mathrm{CHCl}_{3}, 95 \% e e\right)$.

${ }^{1} \mathbf{H}$ NMR $\left(500 \mathrm{MHz}, \mathrm{CDCl}_{3}, \mathrm{Me}_{4} \mathrm{Si}\right): \delta 7.31-7.20(\mathrm{~m}, 10 \mathrm{H}), 7.10(\mathrm{~d}, J=8.7$ $\mathrm{Hz}, 2 \mathrm{H}), 7.01-6.94(\mathrm{~m}, 2 \mathrm{H}), 6.09(\mathrm{~d}, J=15.2 \mathrm{~Hz}, 1 \mathrm{H}$, diastereotopic proton A, $\left.\mathrm{CH}_{2} \mathrm{NO}_{2}\right), 5.91\left(\mathrm{~d}, J=15.2 \mathrm{~Hz}, 1 \mathrm{H}\right.$, diastereotopic proton $\left.\mathrm{B}, \mathrm{CH}_{2} \mathrm{NO}_{2}\right)$, $4.37(\mathrm{~s}, 1 \mathrm{H}), 4.22\left(\mathrm{~d}, J=13.8 \mathrm{~Hz}, 1 \mathrm{H}\right.$, diastereotopic proton A, $\left.\mathrm{SCH}_{2} \mathrm{Ph}\right), 4.13(\mathrm{~d}, J=13.8 \mathrm{~Hz}, 1 \mathrm{H}$, diastereotopic proton $\left.\mathrm{B}, \mathrm{SCH}_{2} \mathrm{Ph}\right), 4.00\left(\mathrm{~d}, J=14.0 \mathrm{~Hz}, 1 \mathrm{H}\right.$, diastereotopic proton $\left.\mathrm{C}, \mathrm{SCH} \mathrm{H}_{2} \mathrm{Ph}\right), 3.73(\mathrm{~d}$, $J=14.0 \mathrm{~Hz}, 1 \mathrm{H}$, diastereotopic proton $\left.\mathrm{D}, \mathrm{SCH}_{2} \mathrm{Ph}\right)$.

${ }^{13} \mathrm{C}$ NMR $\left(126 \mathrm{MHz}, \mathrm{CDCl}_{3}, \mathrm{Me}_{4} \mathrm{Si}\right): \delta$ 189.02, 188.59, 135.48, 135.45, 135.30, 129.23, 129.12, $128.81,128.80,128.75,128.71,128.54,127.81,127.72,125.16\left(\mathrm{q},{ }^{1} J_{\mathrm{C}-\mathrm{F}}=287.9 \mathrm{~Hz}\right), 72.95,70.56$, $56.67\left(\mathrm{q},{ }^{2} J_{\mathrm{C}-\mathrm{F}}=25.7 \mathrm{~Hz}\right), 34.78,34.77$.

${ }^{19}$ F NMR $\left(470 \mathrm{MHz}, \mathrm{CDCl}_{3}\right): \delta-61.55(\mathrm{~s}, 3 \mathrm{~F})$.

IR: $v$ 3064, 3032, 2926, 1694, 1562, 1498, 1454, 1372, 1186, $977 \mathrm{~cm}^{-1}$.

HR-MS (ESI): $\mathrm{m} / \mathrm{z}$ calcd. for $\left[\mathrm{C}_{26} \mathrm{H}_{21} \mathrm{ClF}_{3} \mathrm{NO}_{4} \mathrm{~S}_{2}+\mathrm{Na}\right]^{+}:$590.0445; found, 590.0447 .

Enantiomeric excess: $\left(\right.$ CHIRALPAK $^{\circledR} \mathrm{IB}$; Hexane: ${ }^{i} \mathrm{PrOH}=90: 10$; flow rate: $1.0 \mathrm{~mL} / \mathrm{min}$.; $\left.254 \mathrm{~nm}\right)$ : $\mathrm{t}_{\mathrm{S}}=9.76$ min.(minor), $\mathrm{t}_{\mathrm{R}}=15.06$ min.(major); $95 \%$ ee .<smiles>O=C(Br)C(C(=O)Br)C(C[N+](=O)[O-])C(c1ccccc1)(c1ccc(Cl)cc1)C(F)(F)F</smiles>

ent-3f, New Compound, (white oil, $166 \mathrm{mg}$, 97\% yield).

$\mathbf{R}_{\mathbf{f}}=0.17$ (acetone:hexanes, $1: 10 \mathrm{v} / \mathrm{v}$ ).

Enantiomeric excess: $\left(\right.$ CHIRALPAK $^{\circledR} \mathrm{IB}$; Hexane: ${ }^{i} \mathrm{PrOH}=90: 10$; flow rate: $1.0 \mathrm{~mL} / \mathrm{min}$.; $254 \mathrm{~nm}$ ): $\mathrm{t}_{\mathrm{s}}=9.88 \mathrm{~min}$.(major), $\mathrm{t}_{\mathrm{R}}=15.53 \mathrm{~min}$.(minor); $95 \%$ ee.<smiles>O=C(Br)C(C(=O)Cc1ccccc1)C(C[N+](=O)[O-])(c1cccc(Br)c1)C(F)(F)F</smiles>

3g, New Compound, (white oil, $82 \%$ yield, $150 \mathrm{mg}$ ).

$\mathbf{R}_{\mathbf{f}}=0.14$ (acetone:hexanes, $1: 10 \mathrm{v} / \mathrm{v}$ ).

$[\boldsymbol{\alpha}]_{\mathbf{D}^{20}}=-25.1\left(\mathrm{c}=1.0, \mathrm{CHCl}_{3}, 96 \% e e\right)$.

${ }^{1} \mathbf{H}$ NMR $\left(500 \mathrm{MHz}, \mathrm{CDCl}_{3}, \mathrm{Me}_{4} \mathrm{Si}\right): \delta 7.61(\mathrm{~s}, 1 \mathrm{H}), 7.43(\mathrm{~d}, J=8.0 \mathrm{~Hz}, 1 \mathrm{H})$, $7.28(\mathrm{~m}, 9 \mathrm{H}), 7.03(\mathrm{~m}, 3 \mathrm{H}) 6.10(\mathrm{~d}, J=15.4 \mathrm{~Hz}, 1 \mathrm{H}$, diastereotopic proton A, $\left.\mathrm{CH}_{2} \mathrm{NO}_{2}\right), 5.91\left(\mathrm{~d}, J=15.2 \mathrm{~Hz}, 1 \mathrm{H}\right.$, diastereotopic proton $\left.\mathrm{B}, \mathrm{CH}_{2} \mathrm{NO}_{2}\right), 4.38$ $(\mathrm{s}, 1 \mathrm{H}), 4.25\left(\mathrm{~d}, J=13.8 \mathrm{~Hz}, 1 \mathrm{H}\right.$, diastereotopic proton $\left.\mathrm{A}, \mathrm{SCH}_{2} \mathrm{Ph}\right), 4.16(\mathrm{~d}, J=13.8 \mathrm{~Hz}, 1 \mathrm{H}$, 
diastereotopic proton $\left.\mathrm{B}, \mathrm{SCH} \mathrm{H}_{2} \mathrm{Ph}\right), 3.99\left(\mathrm{~d}, J=14.0 \mathrm{~Hz}, 1 \mathrm{H}\right.$, diastereotopic proton $\left.\mathrm{C}, \mathrm{SCH} \mathrm{H}_{2} \mathrm{Ph}\right), 3.85(\mathrm{~d}$, $J=14.0 \mathrm{~Hz}, 1 \mathrm{H}$, diastereotopic proton $\left.\mathrm{D}, \mathrm{SCH}_{2} \mathrm{Ph}\right)$.

${ }^{13}$ C NMR $\left(126 \mathrm{MHz}, \mathrm{CDCl}_{3}, \mathrm{Me}_{4} \mathrm{Si}\right): \delta 189.06,188.56,135.44,135.24,133.04,132.28,130.32$, $130.26,130.24128 .80,128.79,128.73,128.68,127.80,127.70,125.88,125.05$ (q, ${ }^{1} J_{\mathrm{C}-\mathrm{F}}=288.2 \mathrm{~Hz}$ ), $123.04,72.88,70.46,56.72\left(\mathrm{q},{ }^{2} J_{\mathrm{C}-\mathrm{F}}=25.7 \mathrm{~Hz}\right), 34.78$.

${ }^{19} \mathbf{F}$ NMR $\left(470 \mathrm{MHz}, \mathrm{CDCl}_{3}\right): \delta-61.52(\mathrm{~s}, 3 \mathrm{~F})$.

IR: v 3064, 3031, 2924, 1694, 1562, 1496, 1454, 1372, 1190, 982, $874 \mathrm{~cm}^{-1}$.

HR-MS (ESI): $\mathrm{m} / \mathrm{z}$ calcd. for $\left[\mathrm{C}_{26} \mathrm{H}_{21} \mathrm{BrF}_{3} \mathrm{NO}_{4} \mathrm{~S}_{2}+\mathrm{Na}\right]^{+}:$633.9940; found, 633.9941 .

Enantiomeric excess: $\left(\mathrm{CHIRALPAK}^{\circledR} \mathrm{IB}\right.$; Hexane: ${ }^{i} \mathrm{PrOH}=90: 10$; flow rate: $1.0 \mathrm{~mL} / \mathrm{min}$.; $254 \mathrm{~nm}$ ): $\mathrm{t}_{\mathrm{S}}=22.19$ min.(minor), $\mathrm{t}_{\mathrm{R}}=34.87$ min.(major); $96 \%$ ee .<smiles>O=C(Br)C(C(=O)Cc1ccccc1)C(C[N+](=O)[O-])(c1ccc(Br)cc1)C(F)(F)F</smiles>

3h, New Compound, (colorless oil, 94\% yield, $172 \mathrm{mg}$ ).

$\mathbf{R}_{\mathbf{f}}=0.17$ (acetone:hexanes, $1: 10 \mathrm{v} / \mathrm{v}$ ).

$[\alpha]_{\mathbf{D}}{ }^{20}=-6.2\left(\mathrm{c}=1.0, \mathrm{CHCl}_{3}, 95 \% e e\right)$.

${ }^{1} \mathbf{H}$ NMR $\left(500 \mathrm{MHz}, \mathrm{CDCl}_{3}, \mathrm{Me}_{4} \mathrm{Si}\right): \delta 7.31-7.17(\mathrm{~m}, 12 \mathrm{H}), 7.03-6.94(\mathrm{~m}$, $2 \mathrm{H}), 6.09\left(\mathrm{~d}, J=15.3 \mathrm{~Hz}, 1 \mathrm{H}\right.$, diastereotopic proton $\left.\mathrm{A}, \mathrm{CH}_{2} \mathrm{NO}_{2}\right), 5.90(\mathrm{~d}, J=$

$15.2 \mathrm{~Hz}, 1 \mathrm{H}$, diastereotopic proton $\left.\mathrm{B}, \mathrm{CH}_{2} \mathrm{NO}_{2}\right), 4.36(\mathrm{~s}, 1 \mathrm{H}), 4.23(\mathrm{~d}, J=$ $13.8 \mathrm{~Hz}, 1 \mathrm{H}$, diastereotopic proton $\left.\mathrm{A}, \mathrm{SCH} \mathrm{H}_{2} \mathrm{Ph}\right), 4.13(\mathrm{~d}, J=13.8 \mathrm{~Hz}, 1 \mathrm{H}$, diastereotopic proton $\mathrm{B}$, $\left.\mathrm{SCH}_{2} \mathrm{Ph}\right), 4.01\left(\mathrm{~d}, J=14.0 \mathrm{~Hz}, 1 \mathrm{H}\right.$, diastereotopic proton $\left.\mathrm{C}, \mathrm{SCH}_{2} \mathrm{Ph}\right), 3.72(\mathrm{~d}, J=14.0 \mathrm{~Hz}, 1 \mathrm{H}$, diastereotopic proton $\left.\mathrm{D}, \mathrm{SCH}_{2} \mathrm{Ph}\right)$.

${ }^{13} \mathrm{C}$ NMR $\left(126 \mathrm{MHz}, \mathrm{CDCl}_{3}, \mathrm{Me}_{4} \mathrm{Si}\right): \delta 189.02,188.55,135.46,135.41,132.09,129.77,128.81$, $128.80,128.76,128.75,127.81,127.73,125.10\left(\mathrm{q},{ }^{1} J_{\mathrm{C}-\mathrm{F}}=288.0 \mathrm{~Hz}\right), 123.57,72.88,70.47,56.73(\mathrm{q}$, $\left.{ }^{2} J_{\mathrm{C}-\mathrm{F}}=25.6 \mathrm{~Hz}\right), 34.80,34.79$.

${ }^{19} \mathbf{F}$ NMR $\left(470 \mathrm{MHz}, \mathrm{CDCl}_{3}\right): \delta-61.52(\mathrm{~s}, 3 \mathrm{~F})$.

IR: $v$ 3064, 3031, 1693, 1561, 1497, 1454, 1372, 1187, 1078, $978 \mathrm{~cm}^{-1}$.

HR-MS (ESI): $\mathrm{m} / \mathrm{z}$ calcd. for $\left[\mathrm{C}_{26} \mathrm{H}_{21} \mathrm{BrF}_{3} \mathrm{NO}_{4} \mathrm{~S}_{2}+\mathrm{Na}\right]^{+}$: 633.9940; found, 633.9946 .

Enantiomeric excess: (CHIRALPAK ${ }^{\circledR} \mathrm{IB}$; Hexane: ${ }^{i} \mathrm{PrOH}=90: 10$; flow rate: $1.0 \mathrm{~mL} / \mathrm{min}$.; $254 \mathrm{~nm}$ ): $\mathrm{t}_{\mathrm{S}}=10.53$ min.(minor), $\mathrm{t}_{\mathrm{R}}=16.02$ min.(major); $95 \%$ ee .<smiles>O=C(Br)C(C(=O)Br)C(C[N+](=O)[O-])(c1ccccc1)C(F)(F)F</smiles>

3i, New Compound, (colorless oil, 91\% yield, $164 \mathrm{mg}$ ).

$\mathbf{R}_{\mathbf{f}}=0.12$ (acetone:hexanes, $1: 10 \mathrm{v} / \mathrm{v}$ ).

$[\alpha]_{\mathbf{D}}{ }^{20}=-35.4\left(\mathrm{c}=1.0, \mathrm{CHCl}_{3}, 95 \%\right.$ ee $)$.

${ }^{1} \mathbf{H}$ NMR $\left(500 \mathrm{MHz}, \mathrm{CDCl}_{3}, \mathrm{Me}_{4} \mathrm{Si}\right): \delta 7.75(\mathrm{~s}, 1 \mathrm{H}), 7.54(\mathrm{t}, J=9.2 \mathrm{~Hz}, 2 \mathrm{H})$, $7.31-7.18(\mathrm{~m}, 9 \mathrm{H}), 6.96(\mathrm{~m}, 2 \mathrm{H}), 6.18(\mathrm{~d}, J=15.2 \mathrm{~Hz}, 1 \mathrm{H}$, diastereotopic proton $\left.\mathrm{A}, \mathrm{CH}_{2} \mathrm{NO}_{2}\right), 5.96(\mathrm{~d}, J=15.2 \mathrm{~Hz}, 1 \mathrm{H}$, diastereotopic proton $\mathrm{B}$, $\left.\mathrm{CH}_{2} \mathrm{NO}_{2}\right), 4.41(\mathrm{~s}, 1 \mathrm{H}), 4.23\left(\mathrm{~d}, J=13.8 \mathrm{~Hz}, 1 \mathrm{H}\right.$, diastereotopic proton A, $\left.\mathrm{SCH}_{2} \mathrm{Ph}\right), 4.15(\mathrm{~d}, J=13.8$ $\mathrm{Hz}, 1 \mathrm{H}$, diastereotopic proton $\left.\mathrm{B}, \mathrm{SCH}_{2} \mathrm{Ph}\right), 3.89\left(\mathrm{~d}, J=13.9 \mathrm{~Hz}, 1 \mathrm{H}\right.$, diastereotopic proton $\left.\mathrm{C}, \mathrm{SCH} \mathrm{C}_{2} \mathrm{Ph}\right)$, $3.82\left(\mathrm{~d}, J=13.9 \mathrm{~Hz}, 1 \mathrm{H}\right.$, diastereotopic proton $\left.\mathrm{D}, \mathrm{SCH}_{2} \mathrm{Ph}\right)$.

${ }^{13} \mathrm{C}$ NMR $\left(126 \mathrm{MHz}, \mathrm{CDCl}_{3}, \mathrm{Me}_{4} \mathrm{Si}\right): \delta 189.10,188.55,135.48,135.22,132.05,131.40\left(\mathrm{q},{ }^{2} J_{\mathrm{C}-\mathrm{F}}=\right.$ $32.7 \mathrm{~Hz}), 130.76,129.54,128.86,128.84,128.73,128.71,127.87,127.77,125.13\left(\mathrm{q},{ }^{1} J_{\mathrm{C}-\mathrm{F}}=288.0 \mathrm{~Hz}\right)$, $125.98\left(\mathrm{q},{ }^{3} J_{\mathrm{C}-\mathrm{F}}=3.5 \mathrm{~Hz}\right), 124.29,123.65\left(\mathrm{q},{ }^{1} J_{\mathrm{C}-\mathrm{F}}=272.7 \mathrm{~Hz}\right), 72.94,70.42,57.01\left(\mathrm{q},{ }^{2} J_{\mathrm{C}-\mathrm{F}}=25.7 \mathrm{~Hz}\right)$, $34.87,34.81$.

${ }^{19}$ F NMR $\left(470 \mathrm{MHz}, \mathrm{CDCl}_{3}\right): \delta-61.66(\mathrm{~s}, 3 \mathrm{~F}),-62.71(\mathrm{~s}, 3 \mathrm{~F})$.

IR: $v$ 3087, 2890, 1695, 1562, 1327, 1220, 1157, 1082, $976 \mathrm{~cm}^{-1}$.

HR-MS (ESI): $\mathrm{m} / \mathrm{z}$ calcd. for $\left[\mathrm{C}_{27} \mathrm{H}_{21} \mathrm{~F}_{6} \mathrm{NO}_{4} \mathrm{~S}_{2}+\mathrm{Na}\right]^{+}:$624.0708; found, 624.0711.

Enantiomeric excess: (CHIRALPAK ${ }^{\circledR} \mathrm{IB}$; Hexane: ${ }^{i} \mathrm{PrOH}=90: 10$; flow rate: $1.0 \mathrm{~mL} / \mathrm{min}$.; $254 \mathrm{~nm}$ ): $\mathrm{t}_{\mathrm{S}}=10.24$ min.(minor), $\mathrm{t}_{\mathrm{R}}=18.29$ min.(major); $95 \%$ ee . 
<smiles>O=C(CBr)C(C(=O)Cc1ccccc1)C(c1ccc(C(F)(F)F)cc1)([N+](=O)[O-])C(F)(F)F</smiles>

3j, New Compound, (colorless oil, $84 \%$ yield, $151 \mathrm{mg}$ ).

$\mathbf{R}_{\mathbf{f}}=0.16$ (acetone:hexanes, $\left.1: 10 \mathrm{v} / \mathrm{v}\right)$.

$[\boldsymbol{\alpha}]_{\mathrm{D}}{ }^{20}=-4.4\left(\mathrm{c}=1.0, \mathrm{CHCl}_{3}, 95 \%\right.$ ee $)$.

${ }^{1} \mathbf{H}$ NMR $\left(500 \mathrm{MHz}, \mathrm{CDCl}_{3}, \mathrm{Me}_{4} \mathrm{Si}\right): \delta 7.45(\mathrm{~d}, J=8.5 \mathrm{~Hz}, 2 \mathrm{H}), 7.38(\mathrm{~d}, J=$ $8.6 \mathrm{~Hz}, 2 \mathrm{H}), 7.32-7.19(\mathrm{~m}, 8 \mathrm{H}), 6.98(\mathrm{~m}, 2 \mathrm{H}), 6.15(\mathrm{~d}, J=15.7 \mathrm{~Hz}, 1 \mathrm{H}$, diastereotopic proton $\left.\mathrm{A}, \mathrm{CH}_{2} \mathrm{NO}_{2}\right), 5.96(\mathrm{~d}, J=15.3 \mathrm{~Hz}, 1 \mathrm{H}$, diastereotopic proton $\left.\mathrm{B}, \mathrm{CH}_{2} \mathrm{NO}_{2}\right), 4.36(\mathrm{~s}, 1 \mathrm{H}), 4.24\left(\mathrm{~d}, J=13.8 \mathrm{~Hz}, 1 \mathrm{H}\right.$, diastereotopic proton $\left.\mathrm{A}, \mathrm{SCH} \mathrm{H}_{2} \mathrm{Ph}\right), 4.14(\mathrm{~d}$, $J=13.9 \mathrm{~Hz}, 1 \mathrm{H}$, diastereotopic proton $\left.\mathrm{B}, \mathrm{SCH}_{2} \mathrm{Ph}\right), 4.00(\mathrm{~d}, J=13.9 \mathrm{~Hz}, 1 \mathrm{H}$, diastereotopic proton $\mathrm{C}$, $\left.\mathrm{SCH}_{2} \mathrm{Ph}\right), 3.69\left(\mathrm{~d}, J=13.9 \mathrm{~Hz}, 1 \mathrm{H}\right.$, diastereotopic proton $\left.\mathrm{D}, \mathrm{SCH}_{2} \mathrm{Ph}\right)$.

${ }^{13} \mathrm{C}$ NMR $\left(126 \mathrm{MHz}, \mathrm{CDCl}_{3}, \mathrm{Me}_{4} \mathrm{Si}\right): \delta 189.05,188.50,135.46,135.39,134.73,131.10\left(\mathrm{q},{ }^{2} J_{\mathrm{C}-\mathrm{F}}=\right.$ $33.1 \mathrm{~Hz}), 128.87,128.86,128.80,128.75,127.89,127.82,127.69\left(\mathrm{~d},{ }^{4} J_{\mathrm{C}-\mathrm{F}}=1.3 \mathrm{~Hz}\right), 125.87\left(\mathrm{q},{ }^{3} J_{\mathrm{C}-\mathrm{F}}=\right.$ $3.5 \mathrm{~Hz}), 125.12\left(\mathrm{q},{ }^{1} J_{\mathrm{C}-\mathrm{F}}=288.5 \mathrm{~Hz}\right), 123.49\left(\mathrm{q},{ }^{1} J_{\mathrm{C}-\mathrm{F}}=272.9 \mathrm{~Hz}\right), 72.83,70.32,56.95\left(\mathrm{q},{ }^{2} J_{\mathrm{C}-\mathrm{F}}=25.7\right.$ $\mathrm{Hz}), 34.87,34.86$.

${ }^{19}$ F NMR (470 MHz, $\left.\mathrm{CDCl}_{3}\right): \delta-61.32(\mathrm{~s}, 3 \mathrm{~F}),-62.81(\mathrm{~s}, 3 \mathrm{~F})$.

IR: $v$ 3065, 3033, 1694, 1563, 1329, 1221, 1179, 1131, 1073, $976 \mathrm{~cm}^{-1}$.

HR-MS (ESI): $\mathrm{m} / \mathrm{z}$ calcd. for $\left[\mathrm{C}_{27} \mathrm{H}_{21} \mathrm{~F}_{6} \mathrm{NO}_{4} \mathrm{~S}_{2}+\mathrm{Na}\right]^{+}$: 624.0708; found, 624.0707.

Enantiomeric excess: $\left(\right.$ CHIRALPAK $^{\circledR} \mathrm{IB}$; Hexane: $\mathrm{PrOH}=90: 10$; flow rate: $1.0 \mathrm{~mL} / \mathrm{min}$; $254 \mathrm{~nm}$ ): $\mathrm{t}_{\mathrm{S}}=21.44$ min.(minor), $\mathrm{t}_{\mathrm{R}}=45.55$ min.(major); $95 \%$ ee.<smiles>Cc1cccc(C(Cc2ccccc2)(C[N+](=O)[O-])C(C(=O)Br)C(=O)Br)c1</smiles>

3k, New Compound, (white oil, 79\% yield, $129 \mathrm{mg}$ ).

$\mathbf{R}_{\mathbf{f}}=0.17$ (acetone:hexanes, $1: 10 \mathrm{v} / \mathrm{v}$ ).

$[\alpha]_{\mathrm{D}} \mathbf{2 0}^{\mathbf{2 0}}=-30.2\left(\mathrm{c}=1.0, \mathrm{CHCl}_{3}, 94 \% e e\right)$.

${ }^{1} \mathbf{H}$ NMR $\left(500 \mathrm{MHz}, \mathrm{CDCl}_{3}, \mathrm{Me}_{4} \mathrm{Si}\right): \delta 7.37-7.08(\mathrm{~m}, 12 \mathrm{H}), 6.97(\mathrm{~m}, 2 \mathrm{H})$, $6.15\left(\mathrm{~d}, J=15.3 \mathrm{~Hz}, 1 \mathrm{H}\right.$, diastereotopic proton $\left.\mathrm{A}, \mathrm{CH}_{2} \mathrm{NO}_{2}\right), 5.91(\mathrm{~d}, J=15.3$

$\mathrm{Hz}, 1 \mathrm{H}$, diastereotopic proton $\left.\mathrm{B}, \mathrm{CH}_{2} \mathrm{NO}_{2}\right), 4.45(\mathrm{~s}, 1 \mathrm{H}), 4.25(\mathrm{~d}, J=13.8 \mathrm{~Hz}$,

$1 \mathrm{H}$, diastereotopic proton $\left.\mathrm{A}, \mathrm{SCH} \mathrm{H}_{2} \mathrm{Ph}\right), 4.15\left(\mathrm{~d}, J=13.8 \mathrm{~Hz}, 1 \mathrm{H}\right.$, diastereotopic proton $\left.\mathrm{B}, \mathrm{SCH}_{2} \mathrm{Ph}\right)$, $3.95\left(\mathrm{~d}, J=14.0 \mathrm{~Hz}, 1 \mathrm{H}\right.$, diastereotopic proton $\left.\mathrm{C}, \mathrm{SCH}_{2} \mathrm{Ph}\right), 3.83(\mathrm{~d}, J=14.0 \mathrm{~Hz}, 1 \mathrm{H}$, diastereotopic proton $\left.\mathrm{D}, \mathrm{SCH}_{2} \mathrm{Ph}\right), 2.31$ (s, 3H).

${ }^{13} \mathrm{C}$ NMR $\left(126 \mathrm{MHz}, \mathrm{CDCl}_{3}, \mathrm{Me}_{4} \mathrm{Si}\right): \delta$ 189.28, 188.94, 138.79, 135.61, 135.41, 130.89, 129.80 , $128.84,128.80,128.79,128.65,128.59,127.75,127.60,127.57,125.37$ (q, $\left.{ }^{1} J_{\mathrm{C}-\mathrm{F}}=288.2 \mathrm{~Hz}\right), 124.16$, $73.17,70.95,56.97\left(\mathrm{q},{ }^{2} J_{\mathrm{C}-\mathrm{F}}=25.5 \mathrm{~Hz}\right), 34.70,34.67,21.68$.

${ }^{19}$ F NMR $\left(470 \mathrm{MHz}, \mathrm{CDCl}_{3}\right): \delta-61.11(\mathrm{~s}, 3 \mathrm{~F})$.

IR: $v$ 3063, 3032, 1695, 1562, 1496, 1454, 1373, 1188, 1078, $976 \mathrm{~cm}^{-1}$.

HR-MS (ESI): $\mathrm{m} / \mathrm{z}$ calcd. for $\left[\mathrm{C}_{27} \mathrm{H}_{24} \mathrm{~F}_{3} \mathrm{NO}_{4} \mathrm{~S}_{2}+\mathrm{Na}\right]^{+}:$570.0991; found, 570.0993 .

Enantiomeric excess: (CHIRALPAK ${ }^{\circledR} \mathrm{IB}$; Hexane: $\mathrm{PrOH}=90: 10$; flow rate: $1.0 \mathrm{~mL} / \mathrm{min}$.; $254 \mathrm{~nm}$ ): $\mathrm{t}_{\mathrm{S}}=16.05 \min .\left(\right.$ minor), $\mathrm{t}_{\mathrm{R}}=20.22 \min$.(major); $94 \%$ ee .

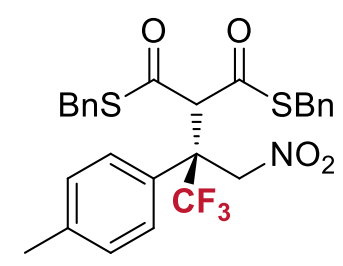

31, New Compound, (colorless oil, 90\% yield, $147 \mathrm{mg}$ ).

$\mathbf{R}_{\mathbf{f}}=0.16($ acetone:hexanes, $1: 10 \mathrm{v} / \mathrm{v})$.

$[\alpha]_{\mathrm{D}}{ }^{20}=-31.5\left(\mathrm{c}=0.2, \mathrm{CHCl}_{3}, 93 \% e e\right)$.

${ }^{1} \mathbf{H}$ NMR $\left(500 \mathrm{MHz}, \mathrm{CDCl}_{3}, \mathrm{Me}_{4} \mathrm{Si}\right): \delta 7.31-7.27(\mathrm{~m}, 2 \mathrm{H}), 7.27-7.22(\mathrm{~m}$, $8 \mathrm{H}), 7.04-6.96(\mathrm{~m}, 4 \mathrm{H}), 6.13(\mathrm{~d}, J=15.1 \mathrm{~Hz}, 1 \mathrm{H}$, diastereotopic proton A, $\left.\mathrm{CH}_{2} \mathrm{NO}_{2}\right), 5.90\left(\mathrm{~d}, J=15.1 \mathrm{~Hz}, 1 \mathrm{H}\right.$, diastereotopic proton $\mathrm{B}, \mathrm{CH}_{2} \mathrm{NO}_{2}$ ), 4.41 (s,

$1 \mathrm{H}), 4.24\left(\mathrm{~d}, J=13.8 \mathrm{~Hz}, 1 \mathrm{H}\right.$, diastereotopic proton $\left.\mathrm{A}, \mathrm{SCH}_{2} \mathrm{Ph}\right), 4.14(\mathrm{~d}, J=13.8 \mathrm{~Hz}, 1 \mathrm{H}$, diastereotopic proton $\left.\mathrm{B}, \mathrm{SCH} \mathrm{H}_{2} \mathrm{Ph}\right), 3.99\left(\mathrm{~d}, J=13.9 \mathrm{~Hz}, 1 \mathrm{H}\right.$, diastereotopic proton $\left.\mathrm{C}, \mathrm{SCH} \mathrm{H}_{2} \mathrm{Ph}\right), 3.76(\mathrm{~d}$, $J=13.9 \mathrm{~Hz}, 1 \mathrm{H}$, diastereotopic proton $\left.\mathrm{D}, \mathrm{SCH}_{2} \mathrm{Ph}\right), 2.26$ (s, 3H).

${ }^{13} \mathrm{C}$ NMR $\left(126 \mathrm{MHz}, \mathrm{CDCl}_{3}, \mathrm{Me}_{4} \mathrm{Si}\right): \delta 189.23,188.90,138.97,135.60,135.60,129.67,128.83$, $128.81,128.80,128.56,127.77,127.57,126.88,126.87,125.39$ (q, $\left.{ }^{1} J_{\mathrm{C}-\mathrm{F}}=287.6 \mathrm{~Hz}\right), 73.13,70.96$, $56.81\left(\mathrm{q},{ }^{2} J_{\mathrm{C}-\mathrm{F}}=25.4 \mathrm{~Hz}\right), 34.72,34.70,20.91$. 
${ }^{19}$ F NMR $\left(470 \mathrm{MHz}, \mathrm{CDCl}_{3}\right): \delta-61.46(\mathrm{~s}, 3 \mathrm{~F})$.

IR: $v$ 3064, 3032, 2928, 2349, 1695, 1562, 1373, 1184, 1085, $976 \mathrm{~cm}^{-1}$.

HR-MS (ESI): $\mathrm{m} / \mathrm{z}$ calcd. for $\left[\mathrm{C}_{27} \mathrm{H}_{24} \mathrm{~F}_{3} \mathrm{NO}_{4} \mathrm{~S}_{2}+\mathrm{Na}\right]^{+}:$570.0991; found, 570.0994 .

Enantiomeric excess: $\left(\right.$ CHIRALPAK $^{\circledR} \mathrm{IB}$; Hexane: ${ }^{i} \mathrm{PrOH}=90: 10$; flow rate: $1.0 \mathrm{~mL} / \mathrm{min}$; $254 \mathrm{~nm}$ ): $\mathrm{t}_{\mathrm{S}}=18.29$ min.(minor), $\mathrm{t}_{\mathrm{R}}=22.89$ min.(major); $93 \%$ ee .<smiles>COc1cccc(C(C[N+](=O)[O-])(C(=O)Br)C(=O)Cc2ccccc2)c1</smiles>

3m, New Compound, (white oil, 90\% yield, $152 \mathrm{mg}$ ).

$\mathbf{R}_{\mathbf{f}}=0.10$ (acetone:hexanes, $1: 10 \mathrm{v} / \mathrm{v}$ ).

$[\alpha]_{\mathbf{D}}{ }^{20}=-20.1\left(\mathrm{c}=1.0, \mathrm{CHCl}_{3}, 95 \% e e\right)$.

${ }^{1} \mathbf{H}$ NMR $\left(500 \mathrm{MHz}, \mathrm{CDCl}_{3}, \mathrm{Me}_{4} \mathrm{Si}\right): \delta 7.32-7.20(\mathrm{~m}, 8 \mathrm{H}), 7.13(\mathrm{t}, J=8.1$ $\mathrm{Hz}, 1 \mathrm{H}), 6.99(\mathrm{~m}, 3 \mathrm{H}), 6.94(\mathrm{~d}, J=8.0 \mathrm{~Hz}, 1 \mathrm{H}), 6.82(\mathrm{~d}, J=8.2 \mathrm{~Hz}, 1 \mathrm{H})$, $6.14\left(\mathrm{~d}, J=15.3 \mathrm{~Hz}, 1 \mathrm{H}\right.$, diastereotopic proton $\left.\mathrm{A}, \mathrm{CH}_{2} \mathrm{NO}_{2}\right), 5.92(\mathrm{~d}, J=$ $15.2 \mathrm{~Hz}, 1 \mathrm{H}$, diastereotopic proton $\left.\mathrm{B}, \mathrm{CH}_{2} \mathrm{NO}_{2}\right), 4.45(\mathrm{~s}, 1 \mathrm{H}), 4.24(\mathrm{~d}, J=13.8 \mathrm{~Hz}, 1 \mathrm{H}$, diastereotopic proton $\left.\mathrm{A}, \mathrm{SCH}_{2} \mathrm{Ph}\right), 4.14\left(\mathrm{~d}, J=13.8 \mathrm{~Hz}, 1 \mathrm{H}\right.$, diastereotopic proton $\left.\mathrm{B}, \mathrm{SCH}_{2} \mathrm{Ph}\right), 3.97(\mathrm{~d}, J=14.0 \mathrm{~Hz}$, $1 \mathrm{H}$, diastereotopic proton $\left.\mathrm{C}, \mathrm{SCH} \mathrm{H}_{2} \mathrm{Ph}\right), 3.82\left(\mathrm{~d}, J=13.9 \mathrm{~Hz}, 1 \mathrm{H}\right.$, diastereotopic proton $\left.\mathrm{D}, \mathrm{SCH} \mathrm{H}_{2} \mathrm{Ph}\right)$, $3.75(\mathrm{~s}, 3 \mathrm{H})$.

${ }^{13} \mathrm{C}$ NMR $\left(126 \mathrm{MHz}, \mathrm{CDCl}_{3}, \mathrm{Me}_{4} \mathrm{Si}\right): \delta 189.23,188.86,159.83,135.58,135.41,132.34,130.02$, $128.82,128.80,128.68,128.60,127.77,127.60,125.27$ (q, $\left.{ }^{1} J_{\mathrm{C}-\mathrm{F}}=287.8 \mathrm{~Hz}\right), 119.30,114.06,113.68$, $73.27,70.79,56.99\left(\mathrm{q},{ }^{2} J_{\mathrm{C}-\mathrm{F}}=25.5 \mathrm{~Hz}\right), 55.32,34.75,34.73$.

${ }^{19}$ F NMR $\left(470 \mathrm{MHz}, \mathrm{CDCl}_{3}\right): \delta-61.25$ (s, 3F).

IR: $v$ 3064, 3032, 1694, 1562, 1496, 1454, 1373, 1183, 1074, $977 \mathrm{~cm}^{-1}$.

HR-MS (ESI): $\mathrm{m} / \mathrm{z}$ calcd. for $\left[\mathrm{C}_{27} \mathrm{H}_{24} \mathrm{~F}_{3} \mathrm{NO}_{5} \mathrm{~S}_{2}+\mathrm{Na}\right]^{+}$: 586.0940; found, 586.0945.

Enantiomeric excess: (CHIRALPAK ${ }^{\circledR} \mathrm{IB}$; Hexane: ${ }^{i} \mathrm{PrOH}=90: 10$; flow rate: $1.0 \mathrm{~mL} / \mathrm{min}$.; $254 \mathrm{~nm}$ ): $\mathrm{t}_{\mathrm{S}}=10.26$ min.(minor), $\mathrm{t}_{\mathrm{R}}=12.77$ min.(major); $95 \%$ ee .<smiles>COc1ccc(C(C[N+](=O)[O-])(C(=O)Br)C(C(=O)Br)C(=O)c2ccccc2)cc1</smiles>

3n, New Compound, (yellow oil, $89 \%$ yield, $150 \mathrm{mg}$ ).

$\mathbf{R}_{\mathbf{f}}=0.08$ (acetone:hexanes, $1: 10 \mathrm{v} / \mathrm{v}$ ).

$[\boldsymbol{\alpha}]_{\mathbf{D}}{ }^{20}=-19.9\left(\mathrm{c}=1.0, \mathrm{CHCl}_{3}, 94 \% e e\right)$.

${ }^{1} \mathbf{H}$ NMR $\left(500 \mathrm{MHz}, \mathrm{CDCl}_{3}, \mathrm{Me}_{4} \mathrm{Si}\right): \delta 7.26(\mathrm{~m}, 10 \mathrm{H}), 6.99(\mathrm{~m}, 2 \mathrm{H}), 6.72$ $(\mathrm{d}, J=8.9 \mathrm{~Hz}, 2 \mathrm{H}), 6.11(\mathrm{~d}, J=15.0 \mathrm{~Hz}, 1 \mathrm{H}$, diastereotopic proton A, $\left.\mathrm{CH}_{2} \mathrm{NO}_{2}\right), 5.89\left(\mathrm{~d}, J=14.9 \mathrm{~Hz}, 1 \mathrm{H}\right.$, diastereotopic proton $\left.\mathrm{B}, \mathrm{CH}_{2} \mathrm{NO}_{2}\right)$, $4.43(\mathrm{~s}, 1 \mathrm{H}), 4.22\left(\mathrm{~d}, J=13.8 \mathrm{~Hz}, 1 \mathrm{H}\right.$, diastereotopic proton A, $\left.\mathrm{SCH}_{2} \mathrm{Ph}\right), 4.13(\mathrm{~d}, J=13.8 \mathrm{~Hz}, 1 \mathrm{H}$, diastereotopic proton $\left.\mathrm{B}, \mathrm{SCH} \mathrm{H}_{2} \mathrm{Ph}\right), 3.98\left(\mathrm{~d}, J=14.0 \mathrm{~Hz}, 1 \mathrm{H}\right.$, diastereotopic proton $\left.\mathrm{C}, \mathrm{SCH} \mathrm{H}_{2} \mathrm{Ph}\right), 3.77(\mathrm{~d}$, $J=14.0 \mathrm{~Hz}, 1 \mathrm{H}$, diastereotopic proton $\left.\mathrm{D}, \mathrm{SCH}_{2} \mathrm{Ph}\right), 3.72(\mathrm{~s}, 3 \mathrm{H})$.

${ }^{13} \mathrm{C}$ NMR $\left(126 \mathrm{MHz}, \mathrm{CDCl}_{3}, \mathrm{Me}_{4} \mathrm{Si}\right): \delta$ 189.29, 188.92, 159.76, 135.64, 135.61, 128.81, 128.79 , $128.77,128.59,128.52,127.75,127.59,125.42\left(\mathrm{q},{ }^{1} J_{\mathrm{C}-\mathrm{F}}=287.8 \mathrm{~Hz}\right), 122.47,114.28,73.37,71.03$, $56.66\left(\mathrm{q},{ }^{2} J_{\mathrm{C}-\mathrm{F}}=25.5 \mathrm{~Hz}\right), 55.17,34.70$.

${ }^{19}$ F NMR $\left(470 \mathrm{MHz}, \mathrm{CDCl}_{3}\right): \delta-61.78(\mathrm{~s}, 3 \mathrm{~F})$.

IR: $v$ 3064, 3030, 2929, 1694, 1561, 1519, 1262, 1190, 1084, $978 \mathrm{~cm}^{-1}$.

HR-MS (ESI): $\mathrm{m} / \mathrm{z}$ calcd. for $\left[\mathrm{C}_{27} \mathrm{H}_{24} \mathrm{~F}_{3} \mathrm{NO}_{5} \mathrm{~S}_{2}+\mathrm{Na}\right]^{+}$: 586.0940 ; found, 586.0943.

Enantiomeric excess: $\left(\mathrm{CHIRALPAK}^{\circledR} \mathrm{IB}\right.$; Hexane: ${ }^{i} \mathrm{PrOH}=90: 10$; flow rate: $1.0 \mathrm{~mL} / \mathrm{min}$;; $254 \mathrm{~nm}$ ): $\mathrm{t}_{\mathrm{S}}=11.44$ min.(minor), $\mathrm{t}_{\mathrm{R}}=13.23$ min.(major); $94 \%$ ee.<smiles>COc1ccc(C(F)(F)C[N+](=O)[O-])c(C(=O)Cc2ccccc2)c1OC1CCCC1</smiles>

3o, New Compound, (yellow oil, $81 \%$ yield, $164 \mathrm{mg}$ ).

$\mathbf{R}_{\mathbf{f}}=0.40$ (acetone:hexanes, $1: 10 \mathrm{v} / \mathrm{v}$ ).

$[\boldsymbol{\alpha}]_{\mathbf{D}}{ }^{20}=-33.7\left(\mathrm{c}=1.0, \mathrm{CHCl}_{3}, 95 \% e e\right)$.

${ }^{1} \mathrm{H}$ NMR $\left(500 \mathrm{MHz}, \mathrm{CDCl}_{3}, \mathrm{Me}_{4} \mathrm{Si}\right): \delta 7.34-7.16(\mathrm{~m}, 8 \mathrm{H}), 6.98(\mathrm{~s}$, $3 \mathrm{H}), 6.91(\mathrm{~d}, J=8.4 \mathrm{~Hz}, 1 \mathrm{H}), 6.63(\mathrm{~d}, J=8.6 \mathrm{~Hz}, 1 \mathrm{H}), 6.15(\mathrm{~d}, J=15.0$ $\mathrm{Hz}, 1 \mathrm{H}$, diastereotopic proton $\left.\mathrm{A}, \mathrm{CH}_{2} \mathrm{NO}_{2}\right), 5.90(\mathrm{~d}, J=15.0 \mathrm{~Hz}, 1 \mathrm{H}$, 
diastereotopic proton $\left.\mathrm{B}, \mathrm{CH}_{2} \mathrm{NO}_{2}\right), 4.71(\mathrm{~s}, 1 \mathrm{H}), 4.47(\mathrm{~s}, 1 \mathrm{H}), 4.23(\mathrm{~d}, J=13.8 \mathrm{~Hz}, 1 \mathrm{H}$, diastereotopic proton $\left.\mathrm{A}, \mathrm{SCH}_{2} \mathrm{Ph}\right), 4.13\left(\mathrm{~d}, J=13.8 \mathrm{~Hz}, 1 \mathrm{H}\right.$, diastereotopic proton $\left.\mathrm{B}, \mathrm{SCH}_{2} \mathrm{Ph}\right), 3.96(\mathrm{~d}, J=13.9 \mathrm{~Hz}$, $1 \mathrm{H}$, diastereotopic proton $\left.\mathrm{C}, \mathrm{SCH}_{2} \mathrm{Ph}\right), 3.83\left(\mathrm{~d}, J=13.9 \mathrm{~Hz}, 1 \mathrm{H}\right.$, diastereotopic proton $\left.\mathrm{D}, \mathrm{SCH}_{2} \mathrm{Ph}\right)$, $3.79(\mathrm{~s}, 3 \mathrm{H}), 2.17-1.74(\mathrm{~m}, 6 \mathrm{H}), 1.59(\mathrm{~s}, 2 \mathrm{H})$.

${ }^{13} \mathrm{C}$ NMR $\left(126 \mathrm{MHz}, \mathrm{CDCl}_{3}, \mathrm{Me}_{4} \mathrm{Si}\right): \delta 189.32,188.97,150.58,147.71,135.68,135.52,128.85$, $128.72,128.69,127.81,127.64,125.47\left(\mathrm{q},{ }^{1} J_{\mathrm{C}-\mathrm{F}}=287.8 \mathrm{~Hz}\right), 122.72,120.00,114.34,111.65,80.85$, $73.62,71.10,56.82\left(\mathrm{q},{ }^{2} J_{\mathrm{C}-\mathrm{F}}=25.4 \mathrm{~Hz}\right), 55.82,34.74,34.73,32.86,32.79,24.18,24.15$.

${ }^{19}$ F NMR $\left(470 \mathrm{MHz}, \mathrm{CDCl}_{3}\right): \delta-61.55(\mathrm{~s}, 3 \mathrm{~F})$.

IR: $v$ 3062, 3031, 2960, 1696, 1562, 1523, 1454, 1373, 1261, 1219, 1186, 1144, $982 \mathrm{~cm}^{-1}$.

HR-MS (ESI): $\mathrm{m} / \mathrm{z}$ calcd. for $\left[\mathrm{C}_{32} \mathrm{H}_{32} \mathrm{~F}_{3} \mathrm{NO}_{6} \mathrm{~S}_{2}+\mathrm{Na}\right]^{+}: 670.1515$; found, 670.1512.

Enantiomeric excess: $\left(\mathrm{CHIRALPAK}^{\circledR} \mathrm{IB}\right.$; Hexane: ${ }^{i} \mathrm{PrOH}=90: 10$; flow rate: $1.0 \mathrm{~mL} / \mathrm{min}$.; $254 \mathrm{~nm}$ ): $\mathrm{t}_{\mathrm{S}}=9.53$ min. $($ minor $), \mathrm{t}_{\mathrm{R}}=15.76$ min.(major); $95 \%$ ee .<smiles>COc1ccc(C(Cc2ccccc2)(C[N+](=O)[O-])C(=O)C(=O)c2ccccc2)cc1OC1CCCC1</smiles>

ent-30, New Compound, (yellow oil, $76 \%$ yield, $154 \mathrm{mg}$ ).

$\mathbf{R}_{\mathbf{f}}=0.40$ (acetone:hexanes, $\left.1: 10 \mathrm{v} / \mathrm{v}\right)$.

Enantiomeric excess: (CHIRALPAK ${ }^{\circledR}$ IB; Hexane: ${ }^{i} \mathrm{PrOH}=90: 10$; flow rate: $1.0 \mathrm{~mL} / \mathrm{min}$.; $254 \mathrm{~nm}$ ): $\mathrm{t}_{\mathrm{S}}=9.57$ min.(major), $\mathrm{t}_{\mathrm{R}}=16.13$ $\min .(\operatorname{minor}) ; 96 \% e e$.<smiles>O=C(Cc1ccccc1)C(C(=O)[Sn]Br)C(CN[N+](=O)[O-])(c1ccccc1)C(F)(F)F</smiles>

3p, New Compound, (colorless oil, $72 \%$ yield, $114 \mathrm{mg}$ ).

$\mathbf{R}_{\mathbf{f}}=0.12$ (acetone:hexanes, $1: 10 \mathrm{v} / \mathrm{v}$ ).

$[\boldsymbol{\alpha}]_{\mathbf{D}}{ }^{20}=+13.1\left(\mathrm{c}=1.0, \mathrm{CHCl}_{3}, 74 \% e e\right)$.

${ }^{1} \mathbf{H}$ NMR $\left(500 \mathrm{MHz}, \mathrm{CDCl}_{3}, \mathrm{Me}_{4} \mathrm{Si}\right): \delta 7.32-7.21(\mathrm{~m}, 8 \mathrm{H}), 7.17-7.12(\mathrm{~m}, 2 \mathrm{H})$, $7.02(\mathrm{~m}, 1 \mathrm{H}), 6.46(\mathrm{~d}, J=3.1 \mathrm{~Hz}, 1 \mathrm{H}), 6.21(\mathrm{dd}, J=3.3,1.8 \mathrm{~Hz}, 1 \mathrm{H}), 5.81(\mathrm{~s}$, $2 \mathrm{H}), 4.54(\mathrm{~s}, 1 \mathrm{H}), 4.21\left(\mathrm{~d}, J=13.8 \mathrm{~Hz}, 1 \mathrm{H}\right.$, diastereotopic proton $\left.\mathrm{A}, \mathrm{SCH}_{2} \mathrm{Ph}\right), 4.12(\mathrm{~d}, J=13.9 \mathrm{~Hz}$, $1 \mathrm{H}$, diastereotopic proton $\left.\mathrm{B}, \mathrm{SCH}_{2} \mathrm{Ph}\right), 4.11\left(\mathrm{~d}, J=13.8 \mathrm{~Hz}, 1 \mathrm{H}\right.$, diastereotopic proton $\left.\mathrm{C}, \mathrm{SCH}_{2} \mathrm{Ph}\right)$, $3.88\left(\mathrm{~d}, J=13.9 \mathrm{~Hz}, 1 \mathrm{H}\right.$, diastereotopic proton $\left.\mathrm{D}, \mathrm{SCH}_{2} \mathrm{Ph}\right)$.

${ }^{13}$ C NMR $\left(126 \mathrm{MHz}, \mathrm{CDCl}_{3}, \mathrm{Me}_{4} \mathrm{Si}\right): \delta 188.30,188.19,143.51,143.27,135.88,135.55,128.97$, $128.81,128.79,128.65,127.76,127.67,124.08\left(\mathrm{q},{ }^{1} J_{\mathrm{C}-\mathrm{F}}=286.8 \mathrm{~Hz}\right), 111.29,110.90,73.25,67.91$, $53.64\left(\mathrm{q},{ }^{2} J_{\mathrm{C}-\mathrm{F}}=27.4 \mathrm{~Hz}\right), 34.67,34.63$.

${ }^{19} \mathbf{F}$ NMR $\left(470 \mathrm{MHz}, \mathrm{CDCl}_{3}\right): \delta-66.67(\mathrm{~s}, 3 \mathrm{~F})$.

IR: $v$ 3064, 3032, 1697, 1562, 1455, 1341, 1193, 1150, $981 \mathrm{~cm}^{-1}$.

HR-MS (ESI): $\mathrm{m} / \mathrm{z}$ calcd. for $\left[\mathrm{C}_{24} \mathrm{H}_{20} \mathrm{~F}_{3} \mathrm{NO}_{5} \mathrm{~S}_{2}+\mathrm{Na}\right]^{+}:$546.0627; found, 546.0630 .

Enantiomeric excess: (CHIRALPAK ${ }^{\circledR} \mathrm{IB}$; Hexane: ${ }^{i} \mathrm{PrOH}=90: 10$; flow rate: $1.0 \mathrm{~mL} / \mathrm{min}$.; $254 \mathrm{~nm}$ ): $\mathrm{t}_{\mathrm{S}}=20.95$ min.(minor), $\mathrm{t}_{\mathrm{R}}=23.14$ min.(major); $74 \%$ ee.<smiles>O=C([Sn]c1ccccc1)C(C(=O)[Sn](=O)[O-])C(C[N+](=O)[O-])(c1cccs1)C(F)(F)F</smiles>

3q, New Compound, (yellow solid, 85\% yield, $137 \mathrm{mg}$ ).

$\mathbf{R}_{\mathbf{f}}=0.13$ (acetone:hexanes, $\left.1: 10 \mathrm{v} / \mathrm{v}\right)$.

mp: $104{ }^{\circ} \mathrm{C}$.

$[\boldsymbol{\alpha}]_{\mathrm{D}}{ }^{20}=-21.9\left(\mathrm{c}=1.0, \mathrm{CHCl}_{3}, 79 \% e e\right)$.

${ }^{1} \mathbf{H}$ NMR $\left(500 \mathrm{MHz}, \mathrm{CDCl}_{3}, \mathrm{Me}_{4} \mathrm{Si}\right): \delta 7.31-7.19(\mathrm{~m}, 9 \mathrm{H}), 7.15(\mathrm{~m}, 1 \mathrm{H}), 7.04$ $(\mathrm{m}, 2 \mathrm{H}), 6.76(\mathrm{~m}, 1 \mathrm{H}), 5.96\left(\mathrm{~d}, J=13.8 \mathrm{~Hz}, 1 \mathrm{H}\right.$, diastereotopic proton $\left.\mathrm{A}, \mathrm{CH}_{2} \mathrm{NO}_{2}\right), 5.92(\mathrm{~d}, J=14.2$ $\mathrm{Hz}, 1 \mathrm{H}$, diastereotopic proton $\left.\mathrm{B}, \mathrm{CH}_{2} \mathrm{NO}_{2}\right), 4.49(\mathrm{~s}, 1 \mathrm{H}), 4.21(\mathrm{~d}, J=13.8 \mathrm{~Hz}, 1 \mathrm{H}$, diastereotopic proton $\left.\mathrm{A}, \mathrm{SCH} \mathrm{H}_{2} \mathrm{Ph}\right), 4.12\left(\mathrm{~d}, J=13.8 \mathrm{~Hz}, 1 \mathrm{H}\right.$, diastereotopic proton $\left.\mathrm{B}, \mathrm{SCH} \mathrm{H}_{2} \mathrm{Ph}\right), 4.01(\mathrm{~d}, J=13.9 \mathrm{~Hz}$, $1 \mathrm{H}$, diastereotopic proton $\left.\mathrm{C}, \mathrm{SCH}_{2} \mathrm{Ph}\right), 3.83\left(\mathrm{~d}, J=13.9 \mathrm{~Hz}, 1 \mathrm{H}\right.$, diastereotopic proton $\left.\mathrm{D}, \mathrm{SCH}_{2} \mathrm{Ph}\right)$.

${ }^{13} \mathrm{C}$ NMR $\left(126 \mathrm{MHz}, \mathrm{CDCl}_{3}, \mathrm{Me}_{4} \mathrm{Si}\right): \delta 188.82,188.52,135.52,135.48,132.05,129.44,128.85$, $128.80,128.79,128.59,127.77,127.63,127.15,126.71,124.64\left(\mathrm{q},{ }^{1} J_{\mathrm{C}-\mathrm{F}}=286.3 \mathrm{~Hz}\right), 74.49,70.82$, $55.35\left(\mathrm{q},{ }^{2} J_{\mathrm{C}-\mathrm{F}}=27.0 \mathrm{~Hz}\right), 34.80,34.72$.

${ }^{19}$ F NMR $\left(470 \mathrm{MHz}, \mathrm{CDCl}_{3}\right): \delta-65.84(\mathrm{~s}, 3 \mathrm{~F})$. 
IR: $v$ 3063, 3033, 2349, 1694, 1562, 1372, 1222, 1191, 1143, $978 \mathrm{~cm}^{-1}$.

HR-MS (ESI): $\mathrm{m} / \mathrm{z}$ calcd. for $\left[\mathrm{C}_{24} \mathrm{H}_{20} \mathrm{~F}_{3} \mathrm{NO}_{4} \mathrm{~S}_{3}+\mathrm{Na}\right]^{+}:$562.0399; found, 562.0401 .

Enantiomeric excess: $\left(\mathrm{CHIRALPAK}^{\circledR} \mathrm{IB}\right.$; Hexane: ${ }^{i} \mathrm{PrOH}=90: 10$; flow rate: $1.0 \mathrm{~mL} / \mathrm{min}$;; $254 \mathrm{~nm}$ ): $\mathrm{t}_{\mathrm{S}}=22.57$ min.(minor), $\mathrm{t}_{\mathrm{R}}=27.40$ min.(major); $79 \%$ ee .
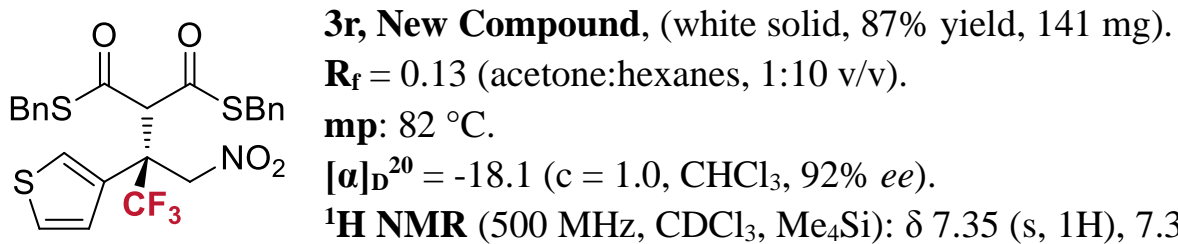

${ }^{1} \mathbf{H}$ NMR $\left(500 \mathrm{MHz}, \mathrm{CDCl}_{3}, \mathrm{Me}_{4} \mathrm{Si}\right): \delta 7.35(\mathrm{~s}, 1 \mathrm{H}), 7.32-7.18(\mathrm{~m}, 8 \mathrm{H}), 7.12(\mathrm{~m}$, $1 \mathrm{H}), 7.09-7.04(\mathrm{~m}, 2 \mathrm{H}), 7.02(\mathrm{~m}, 1 \mathrm{H}), 5.98\left(\mathrm{~d}, J=13.9 \mathrm{~Hz}, 1 \mathrm{H}\right.$, diastereotopic proton A, $\left.\mathrm{CH}_{2} \mathrm{NO}_{2}\right)$, $5.84\left(\mathrm{~d}, J=13.9 \mathrm{~Hz}, 1 \mathrm{H}\right.$, diastereotopic proton $\left.\mathrm{B}, \mathrm{CH}_{2} \mathrm{NO}_{2}\right), 4.41(\mathrm{~s}, 1 \mathrm{H}), 4.22(\mathrm{~d}, J=13.8 \mathrm{~Hz}, 1 \mathrm{H}$, diastereotopic proton $\left.\mathrm{A}, \mathrm{SCH} \mathrm{H}_{2} \mathrm{Ph}\right), 4.12\left(\mathrm{~d}, J=13.8 \mathrm{~Hz}, 1 \mathrm{H}\right.$, diastereotopic proton $\left.\mathrm{B}, \mathrm{SCH} \mathrm{H}_{2} \mathrm{Ph}\right), 4.00(\mathrm{~d}$, $J=13.9 \mathrm{~Hz}, 1 \mathrm{H}$, diastereotopic proton $\left.\mathrm{C}, \mathrm{SCH}_{2} \mathrm{Ph}\right), 3.83(\mathrm{~d}, J=13.9 \mathrm{~Hz}, 1 \mathrm{H}$, diastereotopic proton $\mathrm{D}$, $\left.\mathrm{SCH} \mathrm{H}_{2} \mathrm{Ph}\right)$.

${ }^{13} \mathrm{C}$ NMR $\left(126 \mathrm{MHz}, \mathrm{CDCl}_{3}, \mathrm{Me}_{4} \mathrm{Si}\right): \delta 189.16,188.63,135.53,135.49,130.26,128.84,128.79$, $128.78,128.64,127.75,127.68,126.54,126.41125 .43,125.00\left(\mathrm{q},{ }^{1} J_{\mathrm{C}-\mathrm{F}}=287.7 \mathrm{~Hz}\right), 74.41,69.93$, $54.86\left(\mathrm{q},{ }^{2} J_{\mathrm{C}-\mathrm{F}}=26.4 \mathrm{~Hz}\right), 34.81,34.68$.

${ }^{19}$ F NMR $\left(470 \mathrm{MHz}, \mathrm{CDCl}_{3}\right): \delta-64.98(\mathrm{~s}, 3 \mathrm{~F})$.

IR: $v$ 3119, 3064, 3031, 1694, 1561, 1496, 1411, 1189, 1089, $979 \mathrm{~cm}^{-1}$.

HR-MS (ESI): $\mathrm{m} / \mathrm{z}$ calcd. for $\left[\mathrm{C}_{24} \mathrm{H}_{20} \mathrm{~F}_{3} \mathrm{NO}_{4} \mathrm{~S}_{3}+\mathrm{Na}\right]^{+}:$562.0399; found, 562.0397 .

Enantiomeric excess: $\left(\mathrm{CHIRALPAK}^{\circledR} \mathrm{IB}\right.$; Hexane: ${ }^{i} \mathrm{PrOH}=90: 10$; flow rate: $1.0 \mathrm{~mL} / \mathrm{min}$;; $254 \mathrm{~nm}$ ): $\mathrm{t}_{\mathrm{S}}=24.17$ min.(minor), $\mathrm{t}_{\mathrm{R}}=33.93$ min.(major); $92 \%$ ee.<smiles>O=C(Br)C(C(=O)Cc1ccccc1)C(C[N+](=O)[O-])(C(=O)Br)C(F)(Br)Br</smiles>

3s, New Compound, (white oil, $82 \%$ yield, $135 \mathrm{mg}$ ).

$\mathbf{R}_{\mathbf{f}}=0.24$ (acetone:hexanes, $1: 10 \mathrm{v} / \mathrm{v}$ ).

$[\boldsymbol{\alpha}]_{\mathbf{D}}{ }^{\mathbf{2 0}}=+8.1\left(\mathrm{c}=1.0, \mathrm{CHCl}_{3}, 79 \% e e\right)$.

${ }^{1} \mathbf{H}$ NMR $\left(500 \mathrm{MHz}, \mathrm{CDCl}_{3}, \mathrm{Me}_{4} \mathrm{Si}\right): \delta 7.33-7.16(\mathrm{~m}, 13 \mathrm{H}), 7.09(\mathrm{~d}, J=7.3 \mathrm{~Hz}$, $2 \mathrm{H}), 5.17\left(\mathrm{~d}, J=12.4 \mathrm{~Hz}, 1 \mathrm{H}\right.$, diastereotopic proton A, $\left.\mathrm{CH}_{2} \mathrm{NO}_{2}\right), 5.10(\mathrm{~d}, J=$ $12.5 \mathrm{~Hz}, 1 \mathrm{H}$, diastereotopic proton $\left.\mathrm{B}, \mathrm{CH}_{2} \mathrm{NO}_{2}\right), 4.36(\mathrm{~s}, 1 \mathrm{H}), 4.23-4.03(\mathrm{~m}, 4 \mathrm{H}) 3.64(\mathrm{~d}, J=15.0 \mathrm{~Hz}$, $1 \mathrm{H}$, diastereotopic proton $\left.\mathrm{A}, \mathrm{SCH} \mathrm{H}_{2} \mathrm{Ph}\right), 3.39\left(\mathrm{~d}, J=15.1 \mathrm{~Hz}, 1 \mathrm{H}\right.$, diastereotopic proton $\left.\mathrm{B}, \mathrm{SCH}_{2} \mathrm{Ph}\right)$.

${ }^{13} \mathrm{C}$ NMR $\left(126 \mathrm{MHz}, \mathrm{CDCl}_{3}, \mathrm{Me}_{4} \mathrm{Si}\right): \delta$ 191.01, 190.19, 135.85, 135.60, 132.94, 131.09, 128.91, $128.78,128.76,128.71,128.52,127.83,127.74,127.67,125.46\left(\mathrm{q},{ }^{1} J_{\mathrm{C}-\mathrm{F}}=287.1 \mathrm{~Hz}\right), 75.87,64.27$, $52.03\left(\mathrm{q},{ }^{2} J_{\mathrm{C}-\mathrm{F}}=23.8 \mathrm{~Hz}\right), 35.55,34.61,34.53$.

${ }^{19}$ F NMR $\left(470 \mathrm{MHz}, \mathrm{CDCl}_{3}\right): \delta-65.55(\mathrm{~s}, 3 \mathrm{~F})$.

IR: $v$ 3064, 3032, 2349, 1692, 1558, 1496, 1454, 1173, 1105, 993, $971 \mathrm{~cm}^{-1}$.

HR-MS (ESI): $\mathrm{m} / \mathrm{z}$ calcd. for $\left[\mathrm{C}_{27} \mathrm{H}_{24} \mathrm{~F}_{3} \mathrm{NO}_{4} \mathrm{~S}_{2}+\mathrm{Na}\right]^{+}:$570.0991; found, 570.0994 .

Enantiomeric excess: (CHIRALPAK AD-H; Hexane: ${ }^{i} \mathrm{PrOH}=99: 1$; flow rate: $0.7 \mathrm{~mL} / \mathrm{min}$;; $254 \mathrm{~nm}$ ): $\mathrm{t}_{\mathrm{R}}=32.81$ min.(minor), $\mathrm{t}_{\mathrm{S}}=38.01$ min.(major); $79 \%$ ee.<smiles>CC(C)(C)CC(C[N+](=O)[O-])(C(C(=O)Br)C(=O)Br)C(C(=O)Br)C(=O)Br</smiles>

3t, New Compound, (white oil, 55\% yield, $77 \mathrm{mg}$ ).

$\mathbf{R}_{\mathbf{f}}=0.10$ (acetone:hexanes, $1: 10 \mathrm{v} / \mathrm{v}$ ).

$[\alpha]_{\mathbf{D}}{ }^{20}=+40.4\left(\mathrm{c}=0.25, \mathrm{CHCl}_{3}, 68 \% e e\right)$.

${ }^{1} \mathbf{H} \mathbf{N M R}\left(500 \mathrm{MHz}, \mathrm{CDCl}_{3}, \mathrm{Me}_{4} \mathrm{Si}\right): \delta 7.33-7.18(\mathrm{~m}, 10 \mathrm{H}), 5.37(\mathrm{~d}, J=12.3 \mathrm{~Hz}$, $1 \mathrm{H}$, diastereotopic proton $\left.\mathrm{A}, \mathrm{CH}_{2} \mathrm{NO}_{2}\right), 5.19(\mathrm{~d}, J=12.3 \mathrm{~Hz}, 1 \mathrm{H}$, diastereotopic proton $\left.\mathrm{B}, \mathrm{CH}_{2} \mathrm{NO}_{2}\right), 4.53(\mathrm{~s}, 1 \mathrm{H}), 4.16\left(\mathrm{~m}, 4 \mathrm{H}\right.$, diastereotopic proton, $\left.\mathrm{SCH}_{2} \mathrm{Ph}\right), 1.99(\mathrm{~m}, 1 \mathrm{H}), 1.86(\mathrm{~m}$, $1 \mathrm{H}), 1.83-1.62(\mathrm{~m}, 1 \mathrm{H}), 0.92(\mathrm{~d}, J=6.5 \mathrm{~Hz}, 3 \mathrm{H}) 0.83(\mathrm{~d}, J=6.7 \mathrm{~Hz}, 3 \mathrm{H})$.

${ }^{13} \mathrm{C}$ NMR $\left(126 \mathrm{MHz}, \mathrm{CDCl}_{3}, \mathrm{Me}_{4} \mathrm{Si}\right): \delta$ 190.02, 189.36, 135.72, 135.61, 128.86, 128.80, 128.78, $128.75,127.79,127.71,125.60\left(\mathrm{q},{ }^{1} J_{\mathrm{C}-\mathrm{F}}=286.6 \mathrm{~Hz}\right), 76.61,64.96,51.65\left(\mathrm{q},{ }^{2} J_{\mathrm{C}-\mathrm{F}}=24.9 \mathrm{~Hz}\right), 38.89$, 
34.70, 25.41, 24.09, 23.26.

${ }^{19}$ F NMR (470 MHz, $\left.\mathrm{CDCl}_{3}\right): \delta-66.03(\mathrm{~s}, 3 \mathrm{~F})$.

IR: $v$ 3031, 2970, 1695, 1560, 1219, 1187, 983, $772 \mathrm{~cm}^{-1}$.

HR-MS (ESI): $\mathrm{m} / \mathrm{z}$ calcd. for $\left[\mathrm{C}_{24} \mathrm{H}_{26} \mathrm{~F}_{3} \mathrm{NO}_{4} \mathrm{~S}_{2}+\mathrm{Na}\right]^{+}:$536.1148; found, 536.1149 .

Enantiomeric excess: (CHIRALCEL OJ-H; Hexane: ${ }^{i} \mathrm{PrOH}=95: 5$; flow rate: $0.75 \mathrm{~mL} / \mathrm{min} ; 254 \mathrm{~nm}$ ): $\mathrm{t}_{\mathrm{R}}=23.03 \mathrm{~min}$ (minor), $\mathrm{t}_{\mathrm{s}}=27.12 \mathrm{~min}$ (major); 68\% ee.

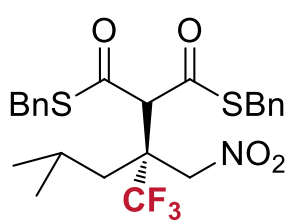

ent-3t, New Compound, (white oil, $55 \%$ yield, $77 \mathrm{mg}$ ).

$\mathbf{R}_{\mathbf{f}}=0.10$ (acetone:hexanes, $1: 10 \mathrm{v} / \mathrm{v}$ ).

Enantiomeric excess: (CHIRALCEL OJ-H; Hexane: ${ }^{\mathrm{PrOH}}=95: 5$; flow rate:

$0.75 \mathrm{~mL} / \mathrm{min} ; 254 \mathrm{~nm}$ ): $\mathrm{t}_{\mathrm{R}}=21.83 \mathrm{~min}$ (major), $\mathrm{t}_{\mathrm{s}}=26.02 \min ($ minor); $67 \%$ ee.<smiles>O=C(Br)C(C(=O)Cc1ccccc1)C(c1ccccc1)([N+](=O)[O-])C(F)(F)F</smiles>

3u, New Compound, (white oil, 97\% yield, $150 \mathrm{mg}$ ).

$\mathbf{R}_{\mathbf{f}}=0.19$ (acetone:hexanes, $\left.1: 10 \mathrm{v} / \mathrm{v}\right)$.

$[\alpha]_{D^{20}}=+6.1\left(\mathrm{c}=1.0, \mathrm{CHCl}_{3}, 89 \% e e\right)$.

${ }^{1} \mathbf{H}$ NMR $\left(500 \mathrm{MHz}, \mathrm{CDCl}_{3}, \mathrm{Me}_{4} \mathrm{Si}\right): \delta 7.36-7.15(\mathrm{~m}, 13 \mathrm{H}), 7.01-6.94(\mathrm{~m}, 2 \mathrm{H})$, $6.73(\mathrm{dd}, J=56.7,53.9 \mathrm{~Hz}, 1 \mathrm{H}), 6.17(\mathrm{~d}, J=16.4 \mathrm{~Hz}, 1 \mathrm{H}$, diastereotopic proton

A, $\left.\mathrm{CH}_{2} \mathrm{NO}_{2}\right), 5.79\left(\mathrm{~d}, J=16.4 \mathrm{~Hz}, 1 \mathrm{H}\right.$, diastereotopic proton $\left.\mathrm{B}, \mathrm{CH}_{2} \mathrm{NO}_{2}\right), 4.49(\mathrm{~s}$,

$1 \mathrm{H}), 4.24\left(\mathrm{~d}, J=13.8 \mathrm{~Hz}, 1 \mathrm{H}\right.$, diastereotopic proton $\left.\mathrm{A}, \mathrm{SCH}_{2} \mathrm{Ph}\right), 4.14(\mathrm{~d}, J=13.8 \mathrm{~Hz}, 1 \mathrm{H}$, diastereotopic proton $\left.\mathrm{B}, \mathrm{SCH}_{2} \mathrm{Ph}\right), 3.95\left(\mathrm{~d}, J=14.0 \mathrm{~Hz}, 1 \mathrm{H}\right.$, diastereotopic proton $\left.\mathrm{C}, \mathrm{SCH} \mathrm{H}_{2} \mathrm{Ph}\right), 3.79(\mathrm{~d}$, $J=14.0 \mathrm{~Hz}, 1 \mathrm{H}$, diastereotopic proton $\mathrm{D}, \mathrm{SCH}_{2} \mathrm{Ph}$ ).

${ }^{13} \mathrm{C}$ NMR $\left(126 \mathrm{MHz}, \mathrm{CDCl}_{3}, \mathrm{Me}_{4} \mathrm{Si}\right): \delta 190.08,189.54,135.88,135.66,133.39\left(\mathrm{~d},{ }^{3} J_{\mathrm{C}-\mathrm{F}}=5.7 \mathrm{~Hz}\right)$, $129.42,128.81,128.75,128.73,128.72,128.54,127.61,127.49,126.03\left(\mathrm{~d},{ }^{4} J_{\mathrm{C}-\mathrm{F}}=4.2 \mathrm{~Hz}\right), 117.16(\mathrm{dd}$, $\left.{ }^{1} J_{\mathrm{C}-\mathrm{F}}=253.0,249.9 \mathrm{~Hz}\right), 74.43\left(\mathrm{~d},{ }^{3} J_{\mathrm{C}-\mathrm{F}}=7.1 \mathrm{~Hz}\right), 68.22\left(\mathrm{~d},{ }^{3} J_{\mathrm{C}-\mathrm{F}}=8.8 \mathrm{~Hz}\right), 53.27\left(\mathrm{t},{ }^{2} J_{\mathrm{C}-\mathrm{F}}=18.1 \mathrm{~Hz}\right)$, 34.52 .

${ }^{19}$ F NMR $\left(470 \mathrm{MHz}, \mathrm{CDCl}_{3}\right): \delta-116.66(\mathrm{~d}, J=278.9 \mathrm{~Hz}, 1 \mathrm{~F}$, diastereotopic fluorine A, $\mathrm{CH} F \mathrm{~F})$, 119.60 (d, $J=278.7 \mathrm{~Hz}, 1 \mathrm{~F}$, diastereotopic fluorine B, CHFF).

IR: $v$ 3063, 3031, 1695, 1556, 1453, 1378, 1327, 1063, 992, $976 \mathrm{~cm}^{-1}$.

HR-MS (ESI): m/z calcd. for $\left[\mathrm{C}_{26} \mathrm{H}_{23} \mathrm{~F}_{2} \mathrm{NO}_{4} \mathrm{~S}_{2}+\mathrm{Na}\right]^{+}:$538.0929; found, 538.0930.

Enantiomeric excess: $\left(\right.$ CHIRALPAK $^{\circledR} \mathrm{IB}$; Hexane: ${ }^{i} \mathrm{PrOH}=90: 10$; flow rate: $1.0 \mathrm{~mL} / \mathrm{min}$;; $254 \mathrm{~nm}$ ): $\mathrm{t}_{\mathrm{S}}=30.94$ min.(major), $\mathrm{t}_{\mathrm{R}}=41.83$ min.(minor); $89 \%$ ee .<smiles>O=C(Br)[C@H]1C(=O)NCC1(c1ccccc1)c1ccccc1</smiles>

5a, New Compound, (white solid, 93\% yield, $656 \mathrm{mg}$ ).

$\mathbf{R}_{\mathbf{f}}=0.39($ EtOAc:hexanes, $1: 2 \mathrm{v} / \mathrm{v})$.

mp: $154{ }^{\circ} \mathrm{C}$.

$[\alpha]_{\mathrm{D}}{ }^{20}=+95.4\left(\mathrm{c}=0.5, \mathrm{CHCl}_{3}, 95 \% e e\right)$.

${ }^{1} \mathbf{H}$ NMR $\left(500 \mathrm{MHz}, \mathrm{CDCl}_{3}, \mathrm{Me}_{4} \mathrm{Si}\right): \delta 7.35-7.26(\mathrm{~m}, 3 \mathrm{H}), 7.25-7.17(\mathrm{~m}, 5 \mathrm{H})$, $7.05(\mathrm{~m}, 2 \mathrm{H}), 5.98(\mathrm{~s}, 1 \mathrm{H}), 4.35(\mathrm{~d}, J=10.5 \mathrm{~Hz}, 1 \mathrm{H}$, diastereotopic proton A, $\left.\mathrm{CH}_{2} \mathrm{NH}\right), 4.18\left(\mathrm{~d}, J=14.0 \mathrm{~Hz}, 1 \mathrm{H}\right.$, diastereotopic proton A, $\left.\mathrm{SCH}_{2} \mathrm{Ph}\right), 4.17(\mathrm{~s}, 1 \mathrm{H}), 4.12(\mathrm{~d}, J=10.1$ $\mathrm{Hz}, 1 \mathrm{H}$, diastereotopic proton $\left.\mathrm{B}, \mathrm{CH}_{2} \mathrm{NH}\right), 3.88\left(\mathrm{~d}, J=14.0 \mathrm{~Hz}, 1 \mathrm{H}\right.$, diastereotopic proton $\left.\mathrm{B}, \mathrm{SCH}_{2} \mathrm{Ph}\right)$. ${ }^{13} \mathrm{C}$ NMR $\left(126 \mathrm{MHz}, \mathrm{CDCl}_{3}, \mathrm{Me}_{4} \mathrm{Si}\right): \delta 192.24,170.63,136.32,133.28,128.78,128.70,128.63$, $128.53,128.18,127.44,126.42\left(\mathrm{q},{ }^{1} J_{\mathrm{C}-\mathrm{F}}=283.7 \mathrm{~Hz}\right), 60.56,55.93\left(\mathrm{q},{ }^{2} J_{\mathrm{C}-\mathrm{F}}=25.2 \mathrm{~Hz}\right), 47.28,33.96$.

${ }^{19}$ F NMR $\left(470 \mathrm{MHz}, \mathrm{CDCl}_{3}\right): \delta-75.99(\mathrm{~s}, 3 \mathrm{~F})$.

IR: $v$ 3232, 1720, 1678, 1266, 1218, 1162, 1065, 757, $701 \mathrm{~cm}^{-1}$.

HR-MS (ESI): $\mathrm{m} / \mathrm{z}$ calcd. for $\left[\mathrm{C}_{19} \mathrm{H}_{16} \mathrm{~F}_{3} \mathrm{NO}_{2} \mathrm{~S}+\mathrm{Na}\right]^{+}: 402.0746$; found, 402.0747 .

Enantiomeric excess: (CHIRALPAK ${ }^{\circledR}$ IA; Hexane: ${ }^{i} \mathrm{PrOH}=90: 10$; flow rate: $1.0 \mathrm{~mL} / \mathrm{min}$.; $254 \mathrm{~nm}$ ): $\mathrm{t}_{\mathrm{R}}=10.15$ min.(major), $\mathrm{t}_{\mathrm{S}}=12.76$ min.(minor); $95 \%$ ee. 
<smiles>O=C(Br)[C@H]1C(=O)NC[C@@]1(c1ccccc1)C(F)(F)F</smiles>

ent-5a, New Compound, (white solid, 93\% yield, $649 \mathrm{mg}$ ).

$\mathbf{R}_{\mathbf{f}}=0.41($ EtOAc:hexanes, $1: 2 \mathrm{v} / \mathrm{v})$.

Enantiomeric excess: $\left(\right.$ CHIRALPAK $^{\circledR}$ IA; Hexane: ${ }^{i} \mathrm{PrOH}=90: 10$; flow rate: 1.0 $\mathrm{mL} / \mathrm{min}$.; $254 \mathrm{~nm}$ ): $\mathrm{t}_{\mathrm{R}}=10.23$ min.(minor), $\mathrm{t}_{\mathrm{s}}=12.57$ min.(major); $95 \%$ ee .<smiles>[B]C(=O)[C@H]1C(=O)NC[C@]1(c1ccc(Cl)cc1)C(F)(F)F</smiles>

5f, New Compound, (white solid, $91 \%$ yield, $715 \mathrm{mg}$ ).

$\mathbf{R}_{\mathbf{f}}=0.61($ EtOAc:hexanes, $1: 2 \mathrm{v} / \mathrm{v})$.

mp: $129^{\circ} \mathrm{C}$.

$[\boldsymbol{\alpha}]_{\mathbf{D}}{ }^{20}=+99.0\left(\mathrm{c}=0.5, \mathrm{CHCl}_{3}, 95 \%\right.$ ee $)$.

${ }^{1} \mathbf{H}$ NMR $\left(500 \mathrm{MHz}, \mathrm{CDCl}_{3}, \mathrm{Me}_{4} \mathrm{Si}\right): \delta 7.22(\mathrm{~m}, 5 \mathrm{H}), 7.06(\mathrm{~m}, 4 \mathrm{H}), 6.73(\mathrm{~s}$, $1 \mathrm{H}), 4.28\left(\mathrm{~d}, J=10.2 \mathrm{~Hz}, 1 \mathrm{H}\right.$, diastereotopic proton A, $\left.\mathrm{CH}_{2} \mathrm{NH}\right), 4.20(\mathrm{~d}, J=$ $14.0 \mathrm{~Hz}, 1 \mathrm{H}$, diastereotopic proton A, $\left.\mathrm{SCH}_{2} \mathrm{Ph}\right), 4.13(\mathrm{~s}, 1 \mathrm{H}), 4.07(\mathrm{~d}, J=9.6 \mathrm{~Hz}, 1 \mathrm{H}$, diastereotopic proton $\left.\mathrm{B}, \mathrm{CH}_{2} \mathrm{NH}\right), 3.86\left(\mathrm{~d}, J=14.0 \mathrm{~Hz}, 1 \mathrm{H}\right.$, diastereotopic proton $\left.\mathrm{B}, \mathrm{SCH}_{2} \mathrm{Ph}\right)$.

${ }^{13} \mathrm{C}$ NMR $\left(126 \mathrm{MHz}, \mathrm{CDCl}_{3}, \mathrm{Me}_{4} \mathrm{Si}\right): \delta 192.08,170.60,136.33,134.90,131.71,129.46,128.81$, $128.80,128.64,127.61,126.23\left(\mathrm{q},{ }^{1} J_{\mathrm{C}-\mathrm{F}}=296.6 \mathrm{~Hz}\right), 60.47,55.47\left(\mathrm{q},{ }^{2} J_{\mathrm{C}-\mathrm{F}}=25.5 \mathrm{~Hz}\right), 47.26,34.05$.

${ }^{19}$ F NMR $\left(470 \mathrm{MHz}, \mathrm{CDCl}_{3}\right): \delta-76.02(\mathrm{~s}, 3 \mathrm{~F})$.

IR: $v 1719,1676,1498,1266,1219,1166,1098,772 \mathrm{~cm}^{-1}$.

HR-MS (ESI): $\mathrm{m} / \mathrm{z}$ calcd. for $\left[\mathrm{C}_{19} \mathrm{H}_{15} \mathrm{ClF}_{3} \mathrm{NO}_{2} \mathrm{~S}+\mathrm{Na}\right]^{+}:$: 436.0356; found, 436.0356 .

Enantiomeric excess: $\left(\mathrm{CHIRALPAK}^{\circledR} \mathrm{IB}\right.$; Hexane: ${ }^{i} \mathrm{PrOH}=95: 5$; flow rate: $1.0 \mathrm{~mL} / \mathrm{min}$;; $254 \mathrm{~nm}$ ): ts $=19.73$ min. $($ minor $), t_{R}=23.74$ min.(major); $95 \%$ ee .<smiles>O=C(CBr)[C@H]1C(=O)NC[C@]1(c1ccc(Cl)cc1)C(F)(F)F</smiles>

ent-5f, New Compound, (white solid, $90 \%$ yield, $723 \mathrm{mg}$ ).

$\mathbf{R}_{\mathbf{f}}=0.61($ EtOAc:hexanes, $1: 2 \mathrm{v} / \mathrm{v})$.

Enantiomeric excess: $\left(\right.$ CHIRALPAK $^{\circledR} \mathrm{IB}$; Hexane: ${ }^{i} \mathrm{PrOH}=95: 5$; flow rate: $1.0 \mathrm{~mL} / \mathrm{min}$.; $254 \mathrm{~nm}$ ): $\mathrm{t}_{\mathrm{S}}=19.11 \mathrm{~min}$.(major), $\mathrm{t}_{\mathrm{R}}=23.90 \mathrm{~min}$.(minor); $94 \%$ ee .<smiles>COc1ccc([C@]2(C(F)(F)F)CNC(=O)[C@H]2C(=O)Br)cc1OC1CCCC1</smiles>

5o, New Compound, (white solid, 97\% yield, $776 \mathrm{mg}$ ).

$\mathbf{R}_{\mathbf{f}}=0.29($ EtOAc:hexanes, $1: 2 \mathrm{v} / \mathrm{v})$.

mp: $101{ }^{\circ} \mathrm{C}$.

$[\boldsymbol{\alpha}]_{\mathbf{D}}{ }^{20}=-5.2\left(\mathrm{c}=0.5, \mathrm{CHCl}_{3}, 95 \% e e\right)$.

${ }^{1} \mathbf{H}$ NMR $\left(500 \mathrm{MHz}, \mathrm{CDCl}_{3}, \mathrm{Me}_{4} \mathrm{Si}\right): \delta 7.25-7.19(\mathrm{~m}, 3 \mathrm{H}), 7.12-7.02$ $(\mathrm{m}, 2 \mathrm{H}), 6.78-6.73(\mathrm{~m}, 1 \mathrm{H}), 6.73-6.64(\mathrm{~m}, 2 \mathrm{H}), 6.23(\mathrm{~s}, 1 \mathrm{H}), 4.59(\mathrm{dd}$, $J=9.6,7.1 \mathrm{~Hz}, 1 \mathrm{H}), 4.33\left(\mathrm{~d}, J=10.2 \mathrm{~Hz}, 1 \mathrm{H}\right.$, diastereotopic proton $\left.\mathrm{A}, \mathrm{CH}_{2} \mathrm{NH}\right), 4.16(\mathrm{~d}, J=13.9 \mathrm{~Hz}$, $1 \mathrm{H}$, diastereotopic proton $\left.\mathrm{A}, \mathrm{SCH} \mathrm{S}_{2} \mathrm{Ph}\right), 4.12(\mathrm{~s}, 1 \mathrm{H}), 4.05(\mathrm{~d}, J=10.1 \mathrm{~Hz}, 1 \mathrm{H}$, diastereotopic proton $\mathrm{B}$, $\left.\mathrm{CH}_{2} \mathrm{NH}\right), 3.94\left(\mathrm{~d}, J=13.9 \mathrm{~Hz}, 1 \mathrm{H}\right.$, diastereotopic proton $\left.\mathrm{B}, \mathrm{SCH}_{2} \mathrm{Ph}\right), 3.84(\mathrm{~s}, 1 \mathrm{H}), 1.92-1.82(\mathrm{~m}$, $2 \mathrm{H}), 1.82-1.73(\mathrm{~m}, 4 \mathrm{H}), 1.60-1.52(\mathrm{~m}, 2 \mathrm{H})$.

${ }^{13} \mathrm{C}$ NMR $\left(126 \mathrm{MHz}, \mathrm{CDCl}_{3}, \mathrm{Me}_{4} \mathrm{Si}\right): \delta$ 192.12, 170.68, 150.22, 147.36, 136.01, 128.71, 128.68 , $127.54,126.55\left(\mathrm{q},{ }^{1} J_{\mathrm{C}-\mathrm{F}}=284.2 \mathrm{~Hz}\right), 125.10,120.45,114.97,111.41,80.45,60.63,55.88,55.53(\mathrm{q}$, $\left.{ }^{2} J_{\mathrm{C}-\mathrm{F}}=25.2 \mathrm{~Hz}\right), 47.25,34.08,33.08,32.57,24.11,24.09$.

${ }^{19}$ F NMR (470 MHz, $\left.\mathrm{CDCl}_{3}\right): \delta-76.12$ (s, 3F).

IR: $v 2964,1724,1680,1519,1263,1219,1179,1114,773 \mathrm{~cm}^{-1}$.

HR-MS (ESI): $\mathrm{m} / \mathrm{z}$ calcd. for $\left[\mathrm{C}_{25} \mathrm{H}_{26} \mathrm{~F}_{3} \mathrm{NO}_{4} \mathrm{~S}+\mathrm{Na}\right]^{+}:$: 516.1427; found, 516.1428.

Enantiomeric excess: $\left(\right.$ CHIRALPAK $^{\circledR} \mathrm{IB}$; Hexane: ${ }^{i} \mathrm{PrOH}=95: 5$; flow rate: $1.0 \mathrm{~mL} / \mathrm{min}$; $\left.254 \mathrm{~nm}\right)$ : $\mathrm{ts}_{\mathrm{S}}$ $=18.20 \mathrm{~min}$.(minor), $\mathrm{t}_{\mathrm{R}}=23.67 \mathrm{~min}$.(major); $95 \%$ ee. 
<smiles>COc1ccc([C@]2(C(F)(F)F)CNC(=O)[C@H]2C(=O)Br)cc1OC1CCCC1</smiles>

ent-5o, New Compound, (yellow solid, 97\% yield, $728 \mathrm{mg}$ ).

$\mathbf{R}_{\mathbf{f}}=0.29($ EtOAc:hexanes, $1: 2 \mathrm{v} / \mathrm{v})$.

Enantiomeric excess: (CHIRALPAK ${ }^{\circledR} \mathrm{IB}$; Hexane: ${ }^{i} \mathrm{PrOH}=95: 5$; flow rate: $1.0 \mathrm{~mL} / \mathrm{min} . ; 254 \mathrm{~nm}$ ): $\mathrm{t}_{\mathrm{s}}=17.88 \mathrm{~min}$.(major), $\mathrm{t}_{\mathrm{R}}=24.10$ min.(minor); $95 \%$ ee.<smiles>CC(C)(C)C[C@]1(C(F)(F)F)CNC(=O)[C@H]1C(=O)Br</smiles>

5t, New Compound, (white solid, $88 \%$ yield, $348 \mathrm{mg}$ ).

$\mathbf{R}_{\mathbf{f}}=0.62(\mathrm{DCM}: \mathrm{MeOH}, 10: 1 \mathrm{v} / \mathrm{v})$.

mp: $104{ }^{\circ} \mathrm{C}$.

$[\alpha]_{\mathbf{D}}{ }^{20}=-3.9\left(\mathrm{c}=1.0, \mathrm{CHCl}_{3}, 83 \% e e\right)$.

${ }^{1} \mathrm{H}$ NMR $\left(500 \mathrm{MHz}, \mathrm{CDCl}_{3}, \mathrm{Me}_{4} \mathrm{Si}\right): \delta 7.75(\mathrm{~s}, 1 \mathrm{H}), 7.31-7.17(\mathrm{~m}, 5 \mathrm{H}), 4.29(\mathrm{~d}, J$ $=13.9 \mathrm{~Hz}, 1 \mathrm{H}$, diastereotopic proton $\left.\mathrm{A}, \mathrm{SCH}_{2} \mathrm{Ph}\right), 4.09(\mathrm{~d}, J=13.9 \mathrm{~Hz}, 1 \mathrm{H}$, diastereotopic proton $\mathrm{B}$, $\left.\mathrm{SCH}_{2} \mathrm{Ph}\right), 3.66(\mathrm{~s}, 1 \mathrm{H}), 3.64\left(\mathrm{~d}, J=11.0 \mathrm{~Hz}, 1 \mathrm{H}\right.$, diastereotopic proton A, $\left.\mathrm{CH}_{2} \mathrm{NH}\right), 3.48(\mathrm{~d}, J=11.0$ $\mathrm{Hz}, 1 \mathrm{H}$, diastereotopic proton $\left.\mathrm{B}, \mathrm{CH}_{2} \mathrm{NH}\right), 1.79(\mathrm{~m}, 2 \mathrm{H}), 1.45(\mathrm{dd}, J=14.5,7.6 \mathrm{~Hz}, 1 \mathrm{H}), 0.84(\mathrm{~d}, J=$ $6.5 \mathrm{~Hz}, 3 \mathrm{H}), 0.78(\mathrm{~d}, J=6.4 \mathrm{~Hz}, 3 \mathrm{H})$.

${ }^{13} \mathrm{C}$ NMR $\left(126 \mathrm{MHz}, \mathrm{CDCl}_{3}, \mathrm{Me}_{4} \mathrm{Si}\right): \delta 193.17,171.75,136.33,128.93,128.71,127.70\left(\mathrm{q},{ }^{1} J_{\mathrm{C}-\mathrm{F}}=\right.$ $284.2 \mathrm{~Hz}), 127.59,60.46,51.37\left(\mathrm{q},{ }^{2} J_{\mathrm{C}-\mathrm{F}}=24.7 \mathrm{~Hz}\right), 48.04,39.14,34.34,24.77,24.56,23.40$.

${ }^{19} \mathbf{F ~ N M R}\left(470 \mathrm{MHz}, \mathrm{CDCl}_{3}\right): \delta-73.85(\mathrm{~s}, 3 \mathrm{~F})$.

IR: $v 3233,2963,1721,1679,1296,1217,1177,1137,756,701 \mathrm{~cm}^{-1}$.

HR-MS (ESI): $\mathrm{m} / \mathrm{z}$ calcd. for $\left[\mathrm{C}_{17} \mathrm{H}_{20} \mathrm{~F}_{3} \mathrm{NO}_{2} \mathrm{~S}+\mathrm{Na}\right]^{+}: 382.1059$; found, 382.1061 .

Enantiomeric excess: $\left(\mathrm{CHIRALPAK}^{\circledR} \mathrm{IB}\right.$; Hexane: $\mathrm{PrOH}=$ 95:5; flow rate: $1.0 \mathrm{~mL} / \mathrm{min}$; $\left.254 \mathrm{~nm}\right): \mathrm{t}_{\mathrm{R}}$ $=12.78 \mathrm{~min} .($ minor $), \mathrm{t}_{\mathrm{S}}=27.30 \mathrm{~min}$.(major); $62 \%$ ee (after recrystallization: $\mathrm{t}_{\mathrm{R}}=12.62 \mathrm{~min}$. (minor), $\mathrm{t}_{\mathrm{s}}$ $=27.20 \mathrm{~min}$. (major); $83 \%$ ee (50\% yield, $174 \mathrm{mg})$ ).<smiles>CC(C)(C)C[C@]1(C(=O)Br)CNC(=O)[C@H]1C(F)(F)F</smiles>

yield, $108 \mathrm{mg})$ ). ent-5t, New Compound, (white solid, $88 \%$ yield, $348 \mathrm{mg}$ ). $\mathbf{R}_{\mathbf{f}}=0.62(\mathrm{DCM}: \mathrm{MeOH}, 10: 1 \mathrm{v} / \mathrm{v})$.

Enantiomeric excess: $\left(\right.$ CHIRALPAK $^{\circledR} \mathrm{IB}$; Hexane: ${ }^{i} \mathrm{PrOH}=95: 5$; flow rate: 1.0 $\mathrm{mL} / \mathrm{min}$; $254 \mathrm{~nm}$ ): $\mathrm{t}_{\mathrm{R}}=12.70 \mathrm{~min}$.(major), $\mathrm{t}_{\mathrm{s}}=27.53 \mathrm{~min}$.(minor); $61 \%$ ee (after recrystallization: $\mathrm{t}_{\mathrm{R}}=12.36 \mathrm{~min}$.(major), $\mathrm{t}_{\mathrm{S}}=28.50 \mathrm{~min} .($ minor $) ; 88 \%$ ee $(31 \%$<smiles>O=C1CC(c2ccccc2)(c2ccccc2)CN1</smiles>

6a, Reported Compound ${ }^{5}$, (white solid, $85 \%$ yield, $337 \mathrm{mg}$ ).

$\mathbf{R}_{\mathbf{f}}=0.48$ (DCM:MeOH, 10:1 v/v).

$[\alpha]_{\mathbf{D}^{20}}=+14.5\left(\mathrm{c}=0.2, \mathrm{CHCl}_{3},>99 \% e e\right)$.

${ }^{1} \mathbf{H}$ NMR (500 MHz, $\left.\mathrm{CDCl}_{3}, \mathrm{Me}_{4} \mathrm{Si}\right): \delta 7.43-7.35(\mathrm{~m}, 3 \mathrm{H}), 7.27$ (m, 3H), 4.16 (d, $J$ $=10.1 \mathrm{~Hz}, 1 \mathrm{H}$, diastereotopic proton $\left.\mathrm{A}, \mathrm{COCH}_{2}\right), 3.87(\mathrm{~d}, J=10.8 \mathrm{~Hz}, 1 \mathrm{H}$, diastereotopic proton $\left.\mathrm{B}, \mathrm{COCH}_{2}\right), 3.08(\mathrm{~d}, J=17.2 \mathrm{~Hz}, 1 \mathrm{H}$, diastereotopic proton A, $\left.\mathrm{CH}_{2} \mathrm{NH}\right), 2.96\left(\mathrm{~d}, J=17.3 \mathrm{~Hz}, 1 \mathrm{H}\right.$, diastereotopic proton $\left.\mathrm{B}, \mathrm{CH}_{2} \mathrm{NH}\right)$.

${ }^{13} \mathrm{C}$ NMR $\left(126 \mathrm{MHz}, \mathrm{CDCl}_{3}, \mathrm{Me}_{4} \mathrm{Si}\right): \delta 175.10,136.90,128.76,128.72,127.81,127.05\left(\mathrm{q},{ }^{1} J_{\mathrm{C}-\mathrm{F}}=\right.$ $282.6 \mathrm{~Hz}), 51.61\left(\mathrm{q},{ }^{2} J_{\mathrm{C}-\mathrm{F}}=24.7 \mathrm{~Hz}\right), 48.67\left(\mathrm{~d},{ }^{3} J_{\mathrm{C}-\mathrm{F}}=2.0 \mathrm{~Hz}\right), 37.64\left(\mathrm{~d},{ }^{3} J_{\mathrm{C}-\mathrm{F}}=1.5 \mathrm{~Hz}\right)$.

${ }^{19}$ F NMR $\left(470 \mathrm{MHz}, \mathrm{CDCl}_{3}\right): \delta-76.73(\mathrm{~s}, 3 \mathrm{~F})$.

IR: $v$ 3224, 2926, 1706, 1310, 1154, 1059, 774, $702 \mathrm{~cm}^{-1}$.

HR-MS (ESI): $\mathrm{m} / \mathrm{z}$ calcd. for $\left[\mathrm{C}_{11} \mathrm{H}_{10} \mathrm{~F}_{3} \mathrm{NO}+\mathrm{Na}\right]^{+}: 252.0607$; found, 252.0607 .

Enantiomeric excess: $\left(\mathrm{CHIRALPAK}^{\circledR} \mathrm{IB}\right.$; Hexane: ${ }^{i} \mathrm{PrOH}=95: 5$; flow rate: $1.0 \mathrm{~mL} / \mathrm{min}$; $\left.210 \mathrm{~nm}\right): \mathrm{t}_{\mathrm{R}}$ $=19.90$ min.(major), $\mathrm{t}_{\mathrm{S}}=21.81 \mathrm{~min}$.(minor); $96 \%$ ee (after recrystallization: $\mathrm{t}_{\mathrm{R}}=17.05$ min.(major), $\mathrm{t}_{\mathrm{s}}$ $=19.85 \mathrm{~min} .($ minor $) ;>99 \%$ ee $(83 \%$ yield, $280 \mathrm{mg})$ ). 
<smiles>O=C1CC(c2ccccc2)(c2ccccc2)CN1</smiles>

yield, $280 \mathrm{mg})$ ).

ent-6a, Reported Compound ${ }^{5}$, (white solid, $84 \%$ yield, $329 \mathrm{mg}$ ).

$\mathbf{R}_{\mathbf{f}}=0.50(\mathrm{DCM}: \mathrm{MeOH}, 10: 1 \mathrm{v} / \mathrm{v})$.

mp: $155^{\circ} \mathrm{C}$.

Enantiomeric excess: $\left(\mathrm{CHIRALPAK}^{\circledR} \mathrm{IB}\right.$; Hexane: ${ }^{i} \mathrm{PrOH}=95: 5$; flow rate: 1.0 $\mathrm{mL} / \mathrm{min}$; $210 \mathrm{~nm}$ ): $\mathrm{t}_{\mathrm{R}}=22.55 \mathrm{~min}$.(minor), $\mathrm{t}_{\mathrm{S}}=24.18 \mathrm{~min}$.(major); $96 \%$ ee (after recrystallization: $\mathrm{t}_{\mathrm{R}}=18.19 \mathrm{~min} .($ minor $), \mathrm{t}_{\mathrm{S}}=20.90 \mathrm{~min} .($ major $) ;>99 \%$ ee $(85 \%$<smiles>O=C1C[C@@](c2ccc(Cl)cc2)(C(F)(F)F)CN1</smiles>

6f, New Compound, (white solid, $84 \%$ yield, $383 \mathrm{mg}$ ).

$\mathbf{R}_{\mathbf{f}}=0.46(\mathrm{DCM}: \mathrm{MeOH}, 10: 1 \mathrm{v} / \mathrm{v})$.

$[\alpha]_{D^{20}}=+25.2\left(\mathrm{c}=0.5, \mathrm{CHCl}_{3},>99 \% e e\right)$.

${ }^{1} \mathbf{H}$ NMR $\left(500 \mathrm{MHz}, \mathrm{CDCl}_{3}, \mathrm{Me}_{4} \mathrm{Si}\right): \delta 7.44-7.35(\mathrm{~m}, 2 \mathrm{H}), 7.22(\mathrm{~d}, J=8.5 \mathrm{~Hz}$, $2 \mathrm{H}), 6.80(\mathrm{~s}, 1 \mathrm{H}), 4.14\left(\mathrm{~d}, J=10.7 \mathrm{~Hz}, 1 \mathrm{H}\right.$, diastereotopic proton $\left.\mathrm{A}, \mathrm{COCH}_{2}\right)$, $3.83\left(\mathrm{~d}, J=10.7 \mathrm{~Hz}, 1 \mathrm{H}\right.$, diastereotopic proton $\left.\mathrm{B}, \mathrm{COCH}_{2}\right), 3.06(\mathrm{~d}, J=17.2$ $\mathrm{Hz}, 1 \mathrm{H}$, diastereotopic proton $\left.\mathrm{A}, \mathrm{CH}_{2} \mathrm{NH}\right), 2.91\left(\mathrm{~d}, J=17.2 \mathrm{~Hz}, 1 \mathrm{H}\right.$, diastereotopic proton $\left.\mathrm{B}, \mathrm{CH}_{2} \mathrm{NH}\right)$. ${ }^{13} \mathrm{C}$ NMR $\left(126 \mathrm{MHz}, \mathrm{CDCl}_{3}, \mathrm{Me}_{4} \mathrm{Si}\right): \delta 174.50,135.29,135.03,129.18,129.03,126.78\left(\mathrm{q},{ }^{1} J_{\mathrm{C}-\mathrm{F}}=\right.$ $282.7 \mathrm{~Hz}), 51.32\left(\mathrm{q},{ }^{2} J_{\mathrm{C}-\mathrm{F}}=26.3 \mathrm{~Hz}\right), 48.46\left(\mathrm{~d},{ }^{3} J_{\mathrm{C}-\mathrm{F}}=2.0 \mathrm{~Hz}\right), 37.48\left(\mathrm{~d},{ }^{3} J_{\mathrm{C}-\mathrm{F}}=1.5 \mathrm{~Hz}\right)$.

${ }^{19}$ F NMR $\left(470 \mathrm{MHz}, \mathrm{CDCl}_{3}\right): \delta-76.73(\mathrm{~s}, 3 \mathrm{~F})$.

IR: $v$ 1711, 1498, 1310, 1163, 1097, 825, 775, $555 \mathrm{~cm}^{-1}$.

HR-MS (ESI): $\mathrm{m} / \mathrm{z}$ calcd. for $\left[\mathrm{C}_{11} \mathrm{H}_{9} \mathrm{ClF}_{3} \mathrm{NO}+\mathrm{Na}\right]^{+}: 286.0217$; found, 286.0222 .

Enantiomeric excess: $\left(\right.$ CHIRALPAK ${ }^{\circledR} \mathrm{IB}$; Hexane: ${ }^{i} \mathrm{PrOH}=95: 5$; flow rate: $1.0 \mathrm{~mL} / \mathrm{min}$; $\left.210 \mathrm{~nm}\right)$ : ts $=18.35$ min. $($ minor $), t_{\mathrm{R}}=26.02$ min.(major); $94 \%$ ee (after recrystallization: $\mathrm{t}_{\mathrm{S}}=19.31 \mathrm{~min}$. (minor), $\mathrm{t}_{\mathrm{R}}$ $=26.79 \mathrm{~min}$. (major); $>99 \%$ ee $(89 \%$ yield, $341 \mathrm{mg})$ ).

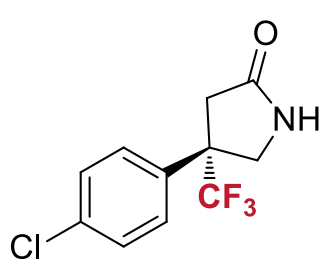

ent-6f, New Compound, (white solid, $85 \%$ yield, $391 \mathrm{mg}$ ).

$\mathbf{R}_{\mathbf{f}}=0.47(\mathrm{DCM}: \mathrm{MeOH}, 10: 1 \mathrm{v} / \mathrm{v})$.

mp: $174{ }^{\circ} \mathrm{C}$.

Enantiomeric excess: $\left(\right.$ CHIRALPAK $^{\circledR} \mathrm{IB}$; Hexane: ${ }^{i} \mathrm{PrOH}=95: 5$; flow rate: $1.0 \mathrm{~mL} / \mathrm{min}$.; $210 \mathrm{~nm}$ ): $\mathrm{t}_{\mathrm{S}}=17.74 \mathrm{~min}$.(major), $\mathrm{t}_{\mathrm{R}}=26.44 \mathrm{~min}$.(minor); $94 \%$ ee (after recrystallization: $\mathrm{t}_{\mathrm{s}}=17.90 \mathrm{~min}$.(major), $\mathrm{t}_{\mathrm{R}}=26.64 \mathrm{~min} .($ minor) $;>99 \%$<smiles>COc1ccc([C@]2(C(F)(F)F)CNC(=O)C2)cc1OC1CCCC1</smiles>

6o, New Compound, (white solid, > 99\% yield, $534 \mathrm{mg}$ ).

$\mathbf{R}_{\mathbf{f}}=0.53$ (DCM:MeOH, 10:1 v/v).

$[\boldsymbol{\alpha}]_{\mathbf{D}}{ }^{20}=+8.0\left(\mathrm{c}=1.0, \mathrm{CHCl}_{3}, 95 \% e e\right)$.

${ }^{1} \mathbf{H}$ NMR $\left(500 \mathrm{MHz}, \mathrm{CDCl}_{3}, \mathrm{Me}_{4} \mathrm{Si}\right): \delta 6.86(\mathrm{~d}, J=8.5 \mathrm{~Hz}, 1 \mathrm{H}), 6.80(\mathrm{dd}$, $J=8.5,2.1 \mathrm{~Hz}, 1 \mathrm{H}), 6.75(\mathrm{~s}, 1 \mathrm{H}), 6.61(\mathrm{~s}, 1 \mathrm{H}), 4.79-4.74(\mathrm{~m}, 1 \mathrm{H}), 4.11$

$\left(\mathrm{d}, J=10.6 \mathrm{~Hz}, 1 \mathrm{H}\right.$, diastereotopic proton A, $\left.\mathrm{COCH}_{2}\right), 3.85(\mathrm{~s}, 3 \mathrm{H}), 3.83$

(s, $1 \mathrm{H}$, diastereotopic proton $\left.\mathrm{B}, \mathrm{COCH}_{2}\right), 3.03\left(\mathrm{~d}, \mathrm{~J}=17.2 \mathrm{~Hz}, 1 \mathrm{H}\right.$, diastereotopic proton $\mathrm{A}, \mathrm{CH}_{2} \mathrm{NH}$ ), $2.93\left(\mathrm{~d}, J=17.2 \mathrm{~Hz}, 1 \mathrm{H}\right.$, diastereotopic proton $\left.\mathrm{B}, \mathrm{CH}_{2} \mathrm{NH}\right), 1.93-1.80(\mathrm{~m}, 6 \mathrm{H}), 1.66-1.59(\mathrm{~m}, 2 \mathrm{H})$.

${ }^{13} \mathrm{C} \mathrm{NMR}\left(126 \mathrm{MHz}, \mathrm{CDCl}_{3}, \mathrm{Me}_{4} \mathrm{Si}\right): \delta 174.87,150.46,147.53,128.93,127.13\left(\mathrm{q},{ }^{1} J_{\mathrm{C}-\mathrm{F}}=282.7 \mathrm{~Hz}\right)$, $120.28,115.19,111.63,80.86,56.02,51.20\left(\mathrm{q},{ }^{2} J_{\mathrm{C}-\mathrm{F}}=26.1 \mathrm{~Hz}\right), 48.66\left(\mathrm{~d},{ }^{3} J_{\mathrm{C}-\mathrm{F}}=1.7 \mathrm{~Hz}\right), 37.68,32.74$, $32.72,24.01$.

${ }^{19}$ F NMR $\left(470 \mathrm{MHz}, \mathrm{CDCl}_{3}\right): \delta-76.83(\mathrm{~s}, 3 \mathrm{~F})$.

IR: $v$ 3267, 2962, 1710, 1518, 1308, 1261, 1177, 1144, $772 \mathrm{~cm}^{-1}$.

HR-MS (ESI): $\mathrm{m} / \mathrm{z}$ calcd. for $\left[\mathrm{C}_{17} \mathrm{H}_{20} \mathrm{~F}_{3} \mathrm{NO}_{3}+\mathrm{Na}\right]^{+}:$366.1288; found, 366.1286.

Enantiomeric excess: $\left(\mathrm{CHIRALPAK}^{\circledR} \mathrm{IA}\right.$; Hexane: ${ }^{i} \mathrm{PrOH}=95: 5$; flow rate: $1.0 \mathrm{~mL} / \mathrm{min}$; $\left.210 \mathrm{~nm}\right): \mathrm{t}_{\mathrm{R}}$ $=17.01 \mathrm{~min}$. (major), $\mathrm{t}_{\mathrm{S}}=23.87 \mathrm{~min}$.(minor); $95 \%$ ee. 
<smiles>COc1ccc([C@]2(C(F)(F)F)CNC(=O)C2)cc1OC1CCCC1</smiles>

ent-6o, New Compound, (white solid, > 99\% yield, $501 \mathrm{mg}$ ).

$\mathbf{R}_{\mathbf{f}}=0.53(\mathrm{DCM}: \mathrm{MeOH}, 10: 1 \mathrm{v} / \mathrm{v})$.

mp: $101{ }^{\circ} \mathrm{C}$.

Enantiomeric excess: $\left(\right.$ CHIRALPAK $^{\circledR}$ IA; Hexane: ${ }^{i} \operatorname{PrOH}=95: 5$; flow rate: $1.0 \mathrm{~mL} / \mathrm{min} . ; 210 \mathrm{~nm}): \mathrm{t}_{\mathrm{R}}=16.84 \min .($ minor $), \mathrm{t}_{\mathrm{S}}=23.33$ min.(major); $95 \% e e$.<smiles>CC(C)C[C@]1(C(F)(F)F)CNC(=O)C1</smiles>

6t, New Compound, (yellow oil, 77\% yield, $78 \mathrm{mg}$ ).

$\mathbf{R}_{\mathbf{f}}=0.51(\mathrm{DCM}: \mathrm{MeOH}, 10: 1 \mathrm{v} / \mathrm{v})$.

$[\alpha]_{\mathrm{D}^{20}}{ }^{20}=+1.5\left(\mathrm{c}=0.2, \mathrm{CHCl}_{3}, 83 \% e e\right)$.

${ }^{1} \mathbf{H}$ NMR $\left(500 \mathrm{MHz}, \mathrm{CDCl}_{3}, \mathrm{Me}_{4} \mathrm{Si}\right): \delta 7.15(\mathrm{~s}, 1 \mathrm{H}), 3.63(\mathrm{~d}, J=10.8 \mathrm{~Hz}, 1 \mathrm{H}$, diastereotopic proton A, $\left.\mathrm{COCH}_{2}\right), 3.30(\mathrm{~d}, J=10.8 \mathrm{~Hz}, 1 \mathrm{H}$, diastereotopic proton $\mathrm{B}$, $\left.\mathrm{COCH}_{2}\right), 2.65\left(\mathrm{~d}, J=17.7 \mathrm{~Hz}, 1 \mathrm{H}\right.$, diastereotopic proton A, $\left.\mathrm{CH}_{2} \mathrm{NH}\right), 2.37(\mathrm{~d}, J=17.7 \mathrm{~Hz}, 1 \mathrm{H}$, diastereotopic proton $\left.\mathrm{B}, \mathrm{CH}_{2} \mathrm{NH}\right), 1.90-1.76(\mathrm{~m}, 1 \mathrm{H}), 1.65(\mathrm{qd}, J=14.6,6.0 \mathrm{~Hz}, 2 \mathrm{H}), 0.99$ (dd, $J=$ $6.6,3.3 \mathrm{~Hz}, 6 \mathrm{H})$.

${ }^{13} \mathrm{C}$ NMR $\left(126 \mathrm{MHz}, \mathrm{CDCl}_{3}, \mathrm{Me}_{4} \mathrm{Si}\right): \delta 175.69,128.15\left(\mathrm{q},{ }^{1} J_{\mathrm{C}-\mathrm{F}}=281.4 \mathrm{~Hz}\right), 47.67\left(\mathrm{~d},{ }^{3} J_{\mathrm{C}-\mathrm{F}}=2.7 \mathrm{~Hz}\right)$, $47.13\left(\mathrm{q},{ }^{2} J_{\mathrm{C}-\mathrm{F}}=25.4 \mathrm{~Hz}\right), 43.08\left(\mathrm{~d},{ }^{3} J_{\mathrm{C}-\mathrm{F}}=1.1 \mathrm{~Hz}\right), 36.84,24.59,24.42$.

${ }^{19}$ F NMR $\left(470 \mathrm{MHz}, \mathrm{CDCl}_{3}\right): \delta-76.30(\mathrm{~s}, 3 \mathrm{~F})$.

IR: $v 2963,1707,1494,1373,1207,1163,684,645 \mathrm{~cm}^{-1}$.

HR-MS (ESI): $\mathrm{m} / \mathrm{z}$ calcd. for $\left[\mathrm{C}_{9} \mathrm{H}_{14} \mathrm{~F}_{3} \mathrm{NO}+\mathrm{Na}\right]^{+}:$: 232.0920; found, 232.0922 .

Enantiomeric excess: $\left(\mathrm{CHIRALPAK}^{\circledR} \mathrm{IB}\right.$; Hexane: ${ }^{i} \mathrm{PrOH}=95: 5$; flow rate: $1.0 \mathrm{~mL} / \mathrm{min}$; $\left.210 \mathrm{~nm}\right): \mathrm{t}_{\mathrm{R}}$ $=8.94$ min. (minor), $\mathrm{t}_{\mathrm{s}}=11.57$ min.(major); $83 \%$ ee .

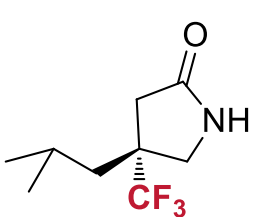

ent-6t, New Compound, (yellow oil, 76\% yield, $48 \mathrm{mg}$ ).

$\mathbf{R}_{\mathbf{f}}=0.51(\mathrm{DCM}: \mathrm{MeOH}, 10: 1 \mathrm{v} / \mathrm{v})$.

Enantiomeric excess: $\left(\right.$ CHIRALPAK ${ }^{\circledR} \mathrm{IB}$; Hexane: ${ }^{i} \mathrm{PrOH}=95: 5$; flow rate: 1.0 $\mathrm{mL} / \mathrm{min}$.; $210 \mathrm{~nm}$ ): $\mathrm{t}_{\mathrm{R}}=8.84$ min.(major), $\mathrm{t}_{\mathrm{S}}=11.62 \min$.(minor); $88 \%$ ee.

7a, Reported Compound ${ }^{5}$, (white solid, $83 \%$ yield, $23 \mathrm{mg}$ ).

$[\alpha]_{D^{20}}=-5.5(\mathrm{c}=0.2, \mathrm{MeOH},>99 \% e e)$.

${ }^{1} \mathbf{H}$ NMR $\left(500 \mathrm{MHz}, \mathrm{D}_{2} \mathrm{O}\right): \delta 7.56-7.41(\mathrm{~m}, 5 \mathrm{H}), 4.08(\mathrm{~d}, J=14.1 \mathrm{~Hz}, 1 \mathrm{H}$, diastereotopic proton A, $\left.\mathrm{COCH}_{2}\right), 3.67(\mathrm{~d}, J=13.9 \mathrm{~Hz}, 1 \mathrm{H}$, diastereotopic proton $\left.\mathrm{B}, \mathrm{COCH}_{2}\right), 3.40\left(\mathrm{~d}, \mathrm{~J}=16.7 \mathrm{~Hz}, 1 \mathrm{H}\right.$, diastereotopic proton $\left.\mathrm{A}, \mathrm{CH}_{2} \mathrm{NH}\right)$,

$3.15\left(\mathrm{~d}, J=16.8 \mathrm{~Hz}, 1 \mathrm{H}\right.$, diastereotopic proton $\left.\mathrm{B}, \mathrm{CH}_{2} \mathrm{NH}\right)$.

${ }^{13} \mathrm{C}$ NMR $\left(126 \mathrm{MHz}, \mathrm{D}_{2} \mathrm{O} / 1,4\right.$-dioxane): $\delta 178.93,138.09,130.43,130.33,129.69,128.77$ (q, ${ }^{1} J_{\mathrm{C}-\mathrm{F}}=$ $282.4 \mathrm{~Hz}), 53.06\left(\mathrm{q},{ }^{2} J_{\mathrm{C}-\mathrm{F}}=25.8 \mathrm{~Hz}\right), 50.03\left(\mathrm{~d},{ }^{3} J_{\mathrm{C}-\mathrm{F}}=1.9 \mathrm{~Hz}\right), 38.83\left(\mathrm{~d},{ }^{3} J_{\mathrm{C}-\mathrm{F}}=1.4 \mathrm{~Hz}\right)$.

${ }^{19}$ F NMR $\left(470 \mathrm{MHz}, \mathrm{D}_{2} \mathrm{O}\right): \delta-74.89(\mathrm{~s}, 3 \mathrm{~F})$.

HR-MS (m/z, ESI): [ $\left.\mathrm{C}_{11} \mathrm{H}_{13} \mathrm{~F}_{3} \mathrm{NO}_{2}\right]^{+}$: calcd., 248.0893; found, 248.0897.

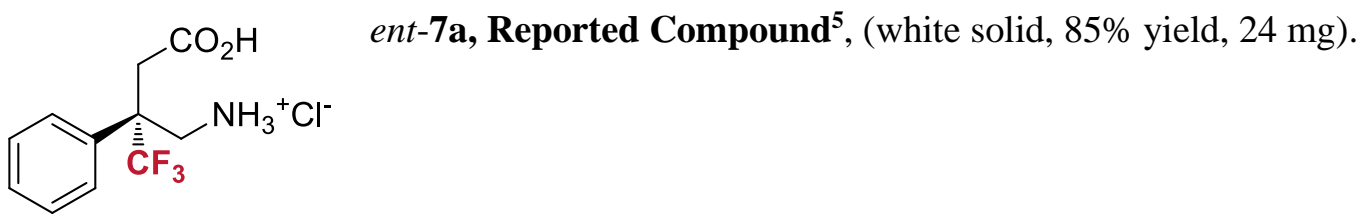


(N)

7f, New Compound, (white solid, $84 \%$ yield, $26 \mathrm{mg}$ ). $[\alpha]_{\mathbf{D}}{ }^{20}=+7.2(\mathrm{c}=0.25, \mathrm{MeOH},>99 \% e e)$.

mp: $164{ }^{\circ} \mathrm{C}$.

${ }^{1} \mathbf{H}$ NMR $\left(500 \mathrm{MHz}, \mathrm{D}_{2} \mathrm{O}\right): \delta 7.48(\mathrm{~s}, 4 \mathrm{H}), 4.01(\mathrm{~d}, J=14.2 \mathrm{~Hz}, 1 \mathrm{H}$, diastereotopic proton A, $\left.\mathrm{COCH}_{2}\right), 3.83(\mathrm{~d}, J=14.2 \mathrm{~Hz}, 1 \mathrm{H}$, diastereotopic proton $\left.\mathrm{B}, \mathrm{COCH}_{2}\right), 3.43\left(\mathrm{~d}, J=17.0 \mathrm{~Hz}, 1 \mathrm{H}\right.$, diastereotopic proton $\left.\mathrm{A}, \mathrm{CH}_{2} \mathrm{NH}\right), 3.21(\mathrm{~d}, J=17.0 \mathrm{~Hz}$, $1 \mathrm{H}$, diastereotopic proton $\left.\mathrm{B}, \mathrm{CH}_{2} \mathrm{NH}\right)$.

${ }^{13}$ C NMR $\left(126 \mathrm{MHz}, \mathrm{D}_{2} \mathrm{O}\right): \delta 174.26,135.06,130.57,129.33,129.30,125.86\left(\mathrm{q},{ }^{1} J_{\mathrm{C}-\mathrm{F}}=283.4 \mathrm{~Hz}\right)$, $48.95\left(\mathrm{q},{ }^{2} J_{\mathrm{C}-\mathrm{F}}=24.5 \mathrm{~Hz}\right), 40.95,36.87$.

${ }^{19}$ F NMR $\left(470 \mathrm{MHz}, \mathrm{D}_{2} \mathrm{O}\right): \delta-73.65(\mathrm{~s}, 3 \mathrm{~F})$.

HR-MS (m/z, ESI): $\left[\mathrm{C}_{11} \mathrm{H}_{12} \mathrm{ClF}_{3} \mathrm{NO}_{2}\right]^{+}$: calcd., 282.0503; found, 282.0507.

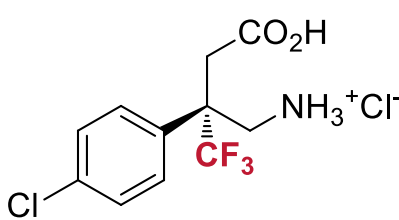

ent-7f, New Compound, (white solid, $86 \%$ yield, $27 \mathrm{mg}$ ).<smiles>CC(C)C[C@](C)(CN)C(=O)O</smiles>

7t, New Compound, (white solid, $81 \%$ yield, $19 \mathrm{mg}$ ). $[\alpha]_{\mathrm{D}}{ }^{20}=-18.7(\mathrm{c}=0.15, \mathrm{MeOH}, 83 \% e e)$.

${ }^{1} \mathbf{H}$ NMR $\left(500 \mathrm{MHz}, \mathrm{D}_{2} \mathrm{O}\right): \delta 3.35(\mathrm{~d}, J=14.0 \mathrm{~Hz}, 1 \mathrm{H}$, diastereotopic proton A, $\left.\mathrm{COCH}_{2}\right), 3.28\left(\mathrm{~d}, J=13.7 \mathrm{~Hz}, 1 \mathrm{H}\right.$, diastereotopic proton $\left.\mathrm{B}, \mathrm{COCH}_{2}\right), 2.69$ (d, $J=15.4 \mathrm{~Hz}, 1 \mathrm{H}$, diastereotopic proton $\left.\mathrm{A}, \mathrm{CH}_{2} \mathrm{NH}\right), 2.63(\mathrm{~d}, J=15.3 \mathrm{~Hz}, 1 \mathrm{H}$, diastereotopic proton B, $\left.\mathrm{CH}_{2} \mathrm{NH}\right), 1.82-1.76(\mathrm{~m}, 1 \mathrm{H}), 1.55(\mathrm{~d}, J=5.4 \mathrm{~Hz}, 2 \mathrm{H}), 0.89(\mathrm{dd}, J=6.6 \mathrm{~Hz}, 6 \mathrm{H})$.

${ }^{13} \mathrm{C}$ NMR $\left(126 \mathrm{MHz}, \mathrm{D}_{2} \mathrm{O} / \mathrm{CD}_{3} \mathrm{OD}\right): \delta 174.42,127.43\left(\mathrm{q},{ }^{1} J_{\mathrm{C}-\mathrm{F}}=284.9 \mathrm{~Hz}\right), 45.24\left(\mathrm{q},{ }^{2} J_{\mathrm{C}-\mathrm{F}}=23.5 \mathrm{~Hz}\right)$, 41.67, 39.72, 35.54, 24.05, 22.87

${ }^{19}$ F NMR $\left(470 \mathrm{MHz}, \mathrm{D}_{2} \mathrm{O}\right): \delta-72.45$ (s, 3F).

HR-MS (m/z, ESI): [ $\left.\mathrm{C}_{9} \mathrm{H}_{17} \mathrm{~F}_{3} \mathrm{NO}_{2}\right]^{+}$: calcd., 228.1206; found, 228.1208.<smiles>CC(C)(C)C[C@](CN[CH]Cl)(CC(=O)O)C(F)(F)F</smiles>
ent-7t, New Compound, (white solid, $83 \%$ yield, $22 \mathrm{mg}$ ). mp: $153{ }^{\circ} \mathrm{C}$ 
6.1. NMR spectra of the products $(3-7)$

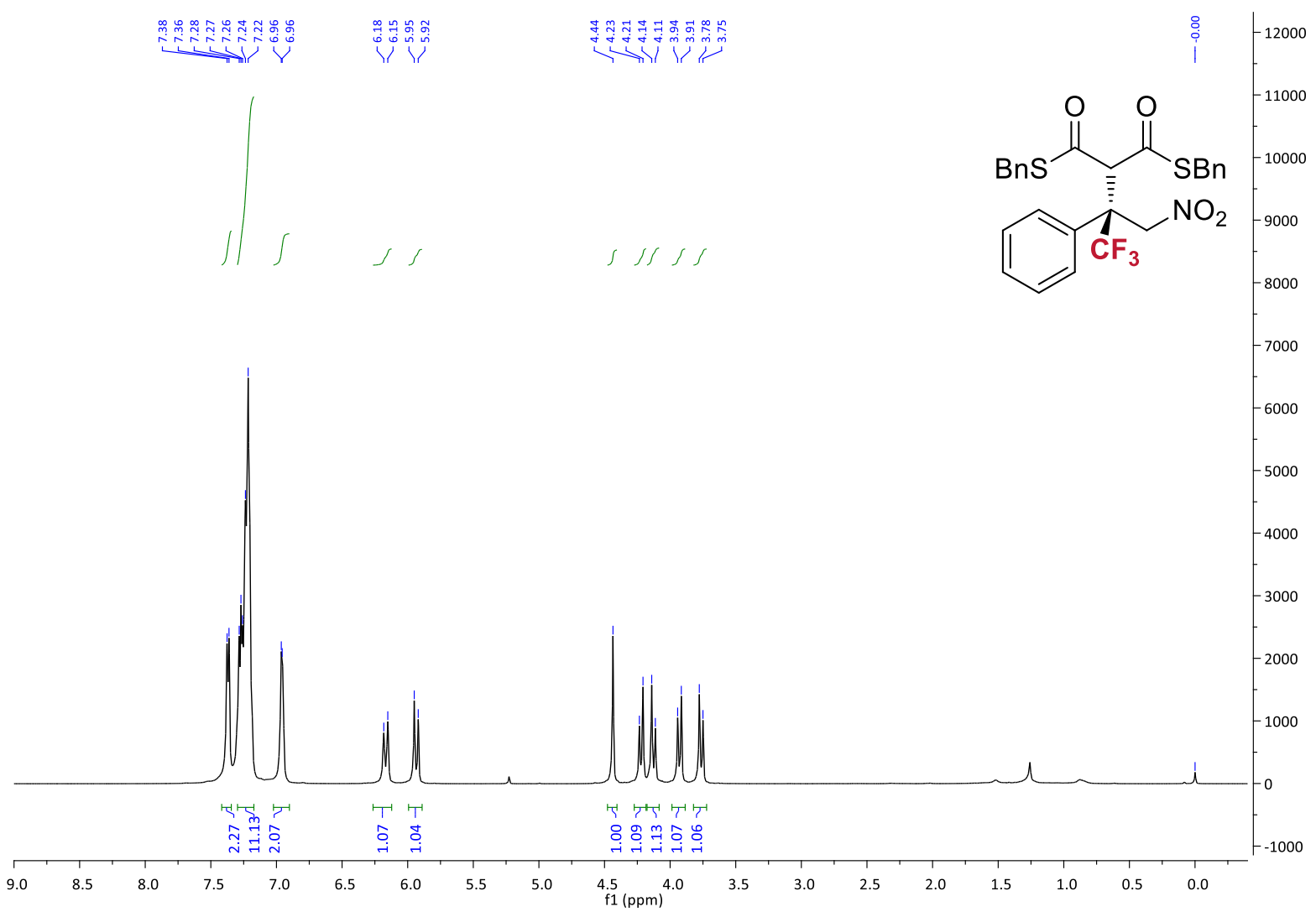

${ }^{1} \mathrm{H}$ NMR spectrum of $\mathbf{3 a b}$

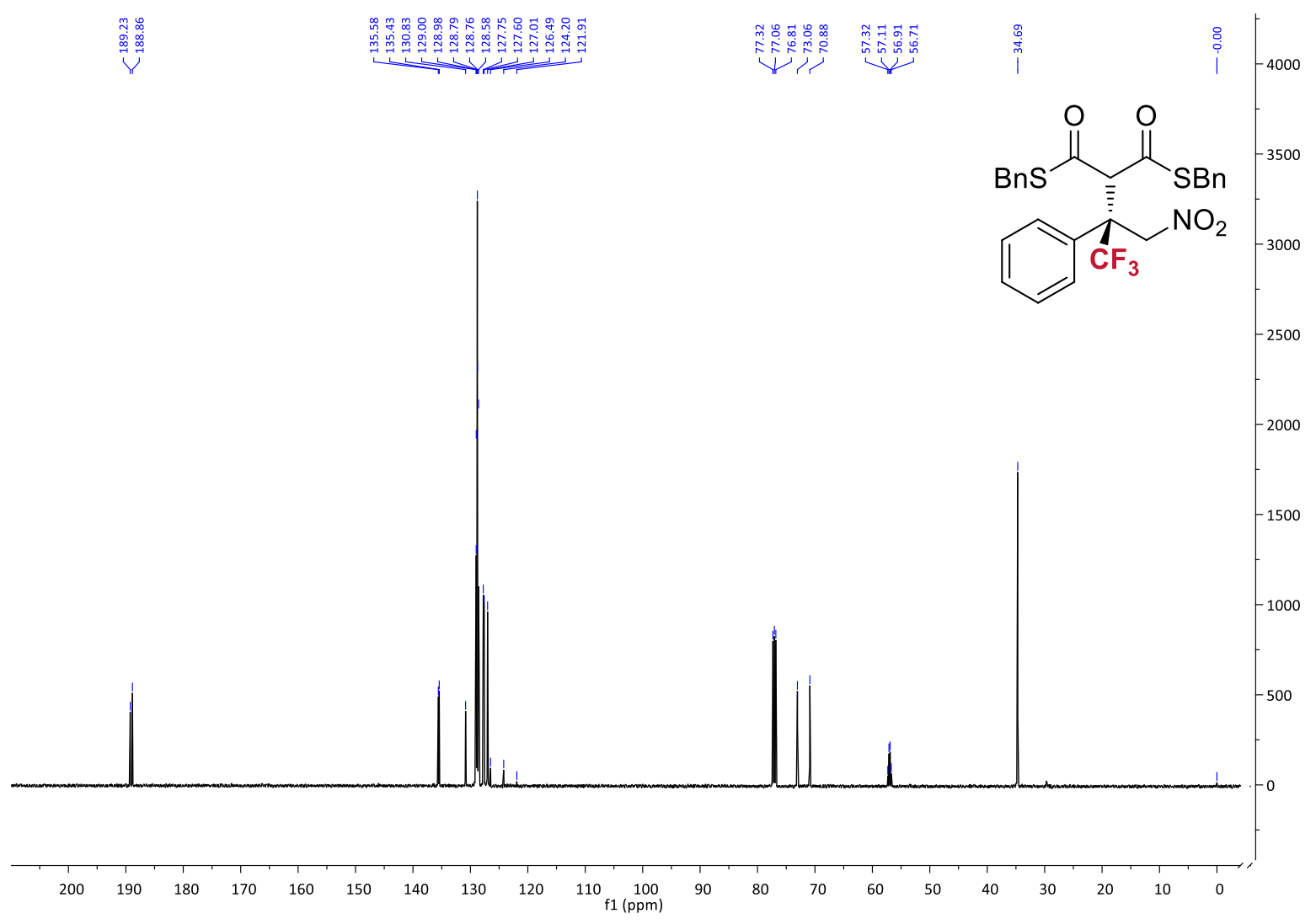

${ }^{13} \mathrm{C}$ NMR spectrum of $\mathbf{3 a b}$ 


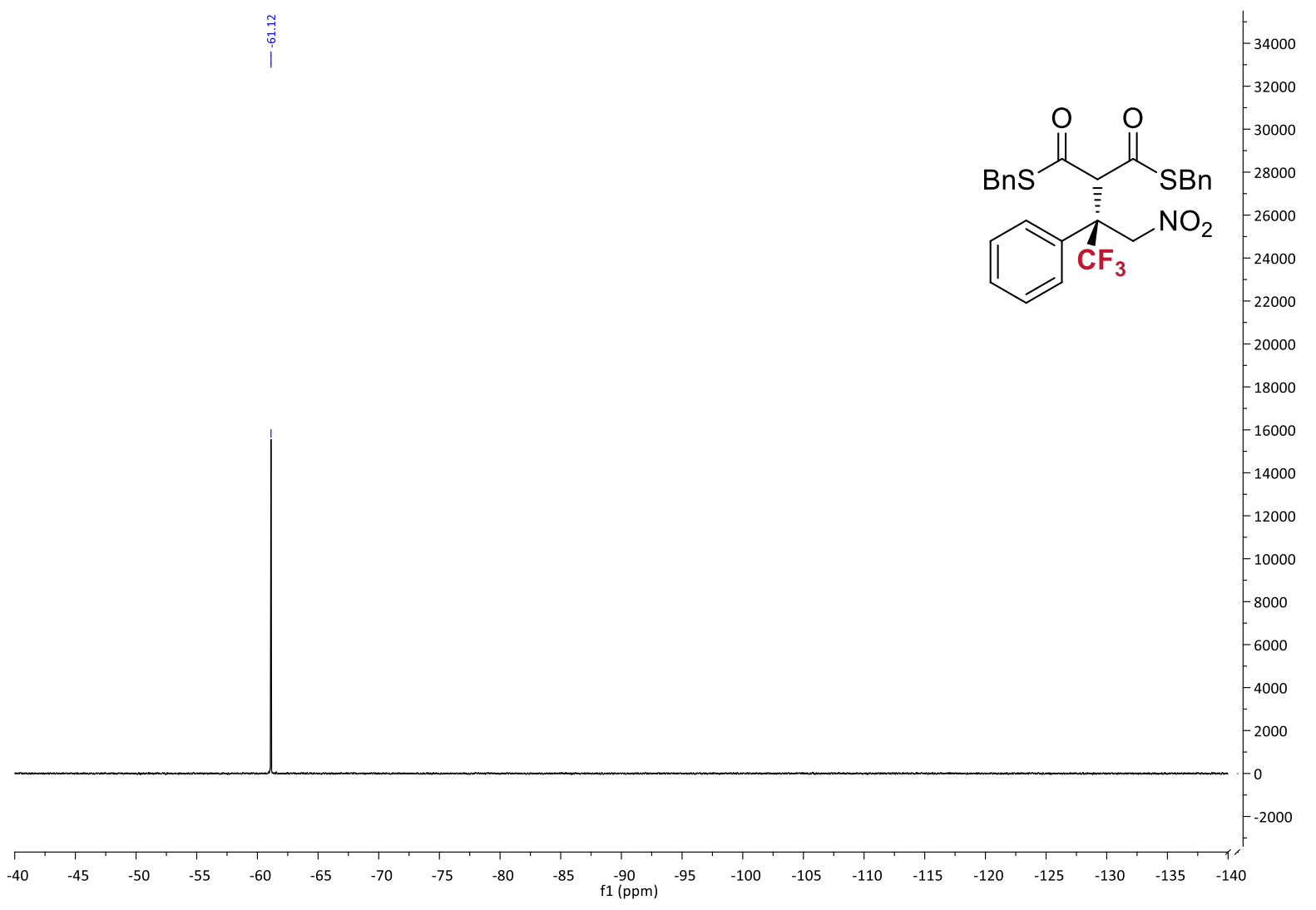

${ }^{19} \mathrm{~F}$ NMR spectrum of $\mathbf{3 a b}$

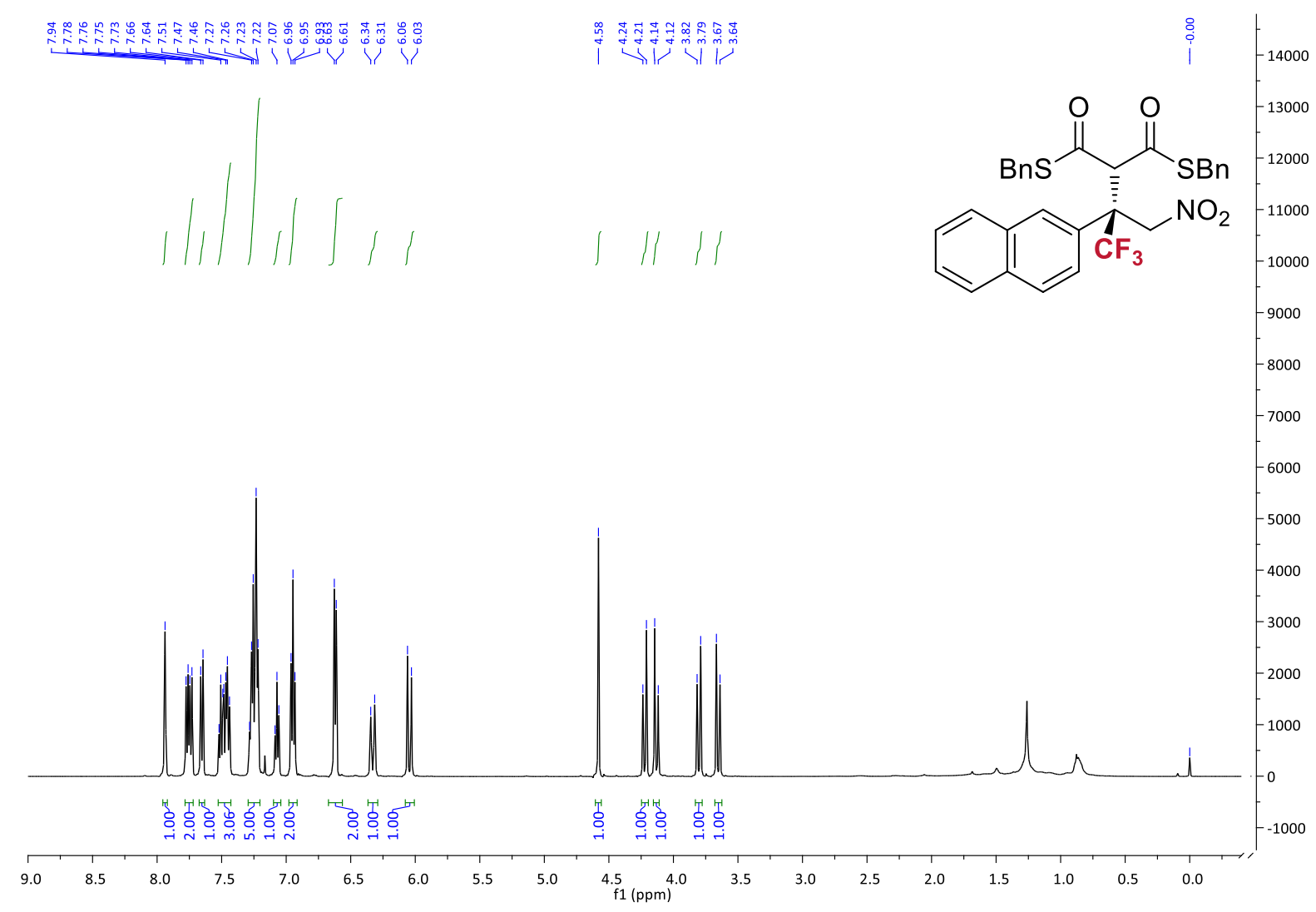

${ }^{1} \mathrm{H}$ NMR spectrum of $\mathbf{3 b}$ 


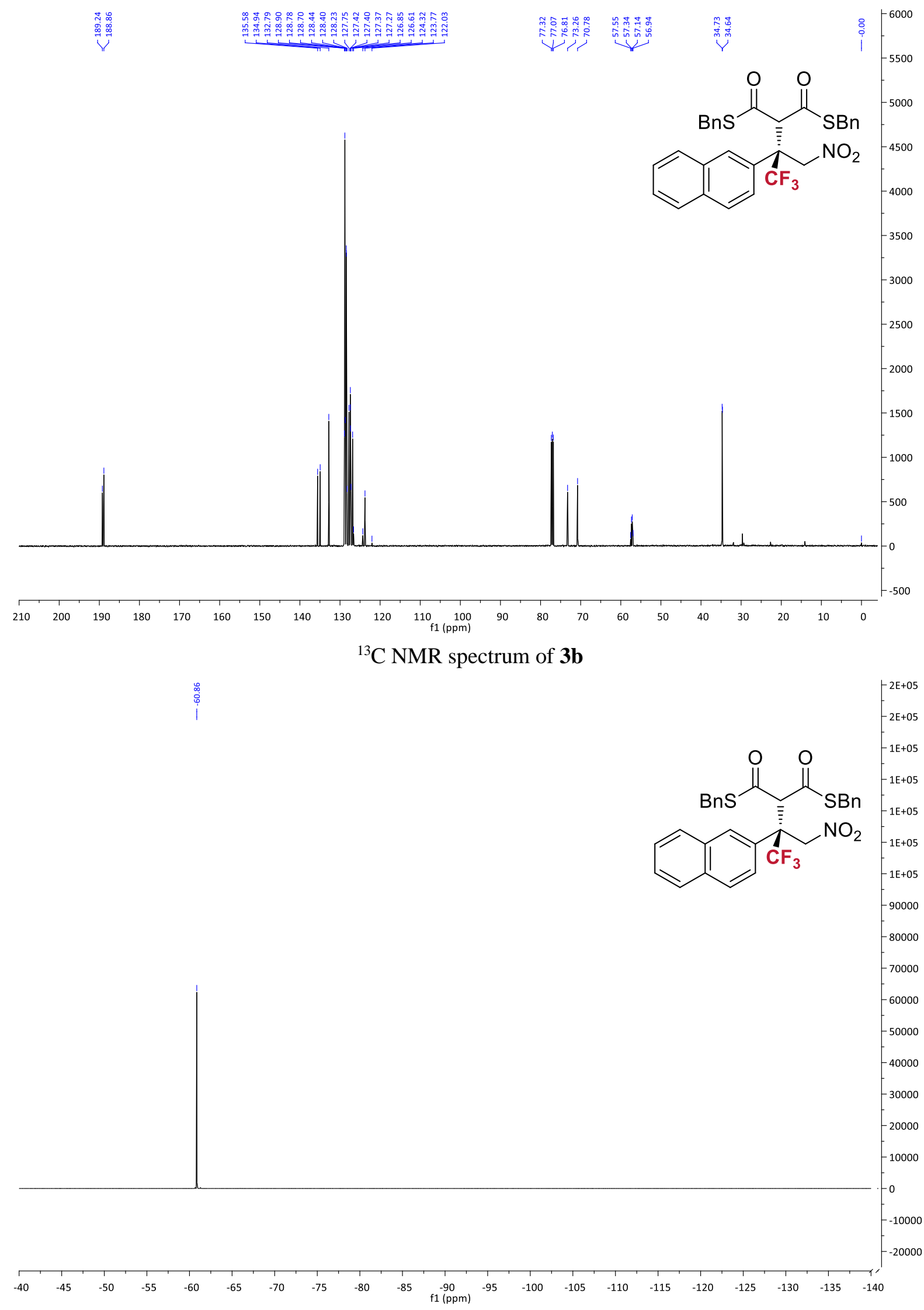

${ }^{19} \mathrm{~F}$ NMR spectrum of $\mathbf{3 b}$ 


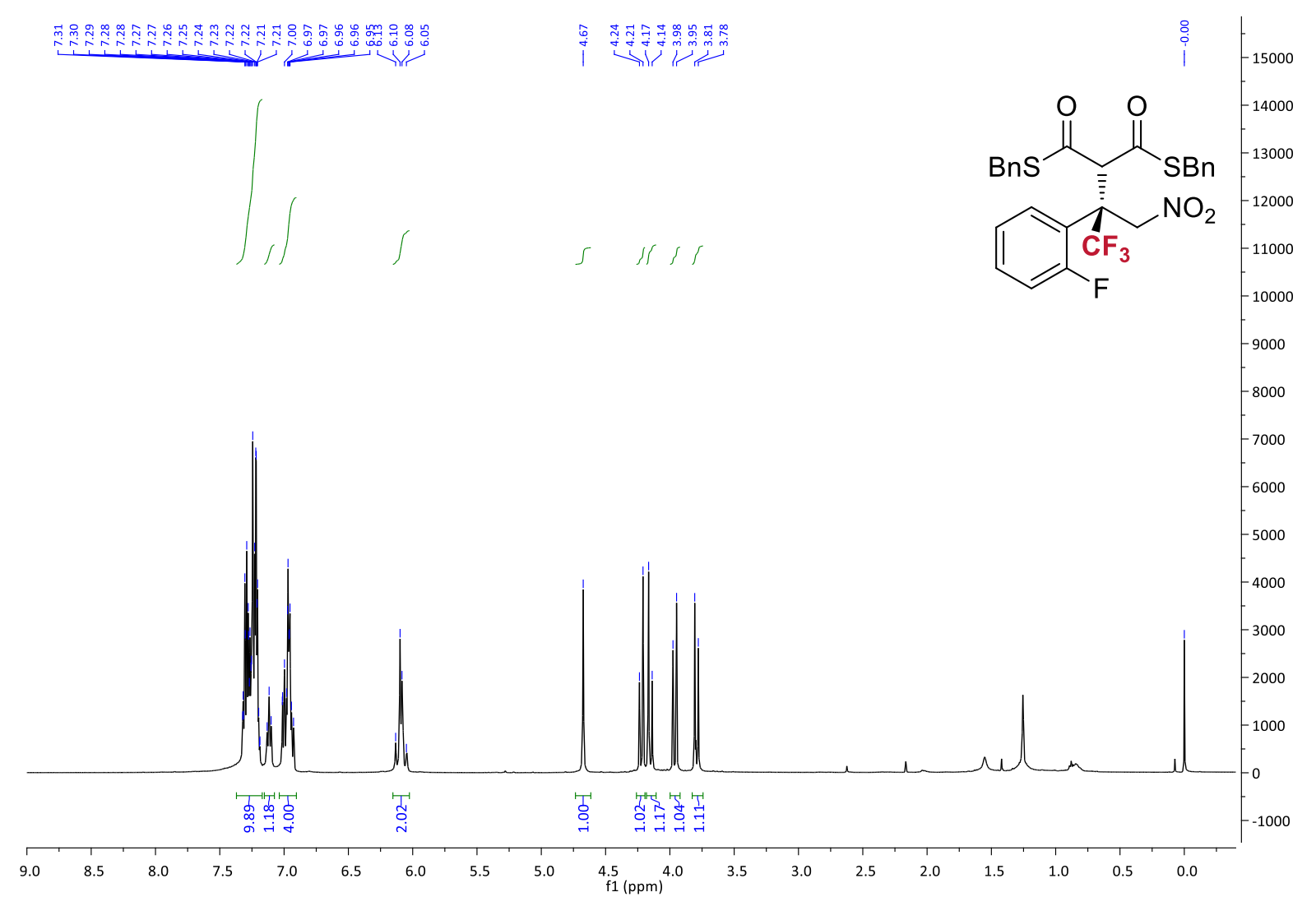

${ }^{1} \mathrm{H}$ NMR spectrum of $\mathbf{3 c}$

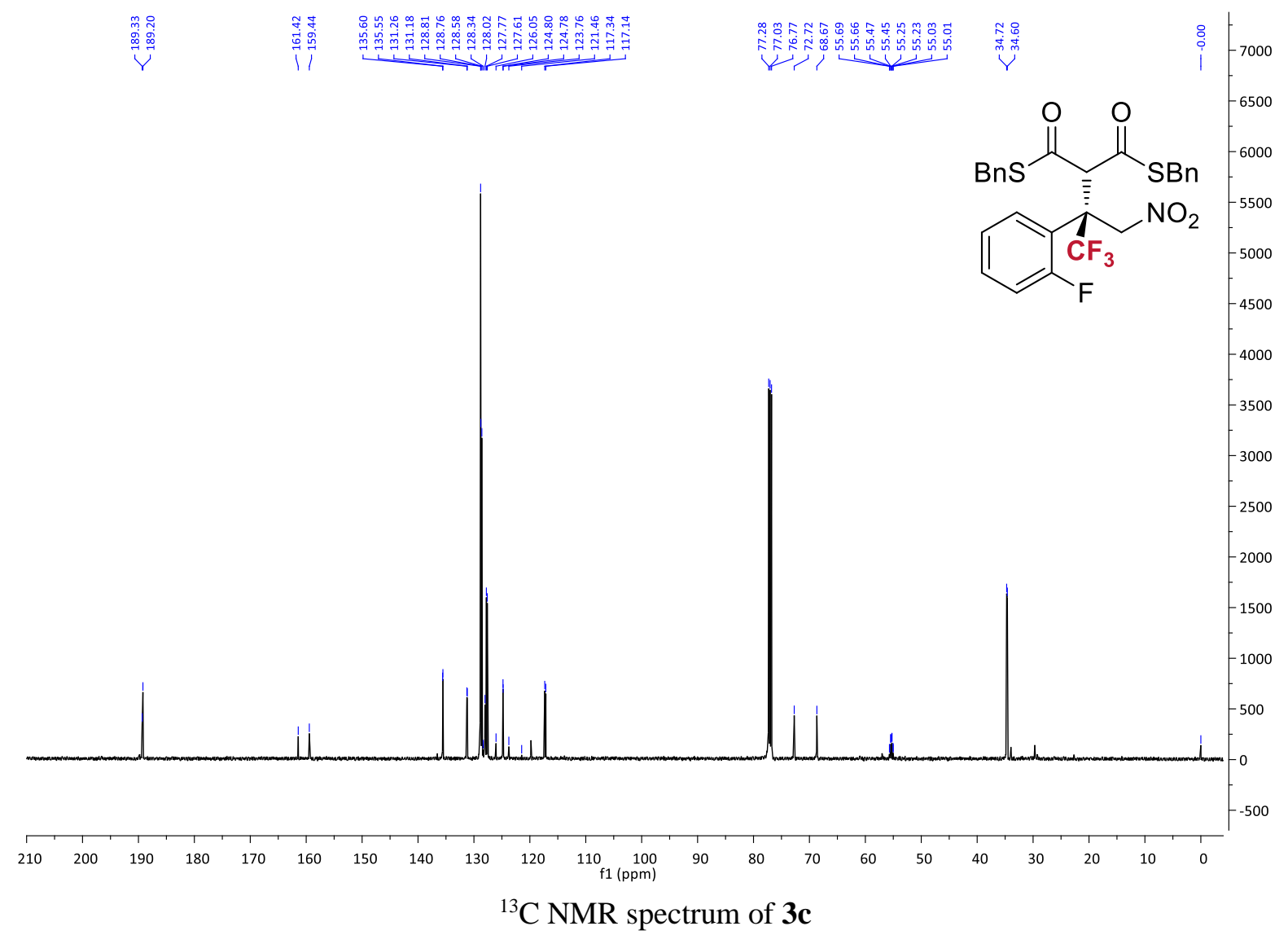




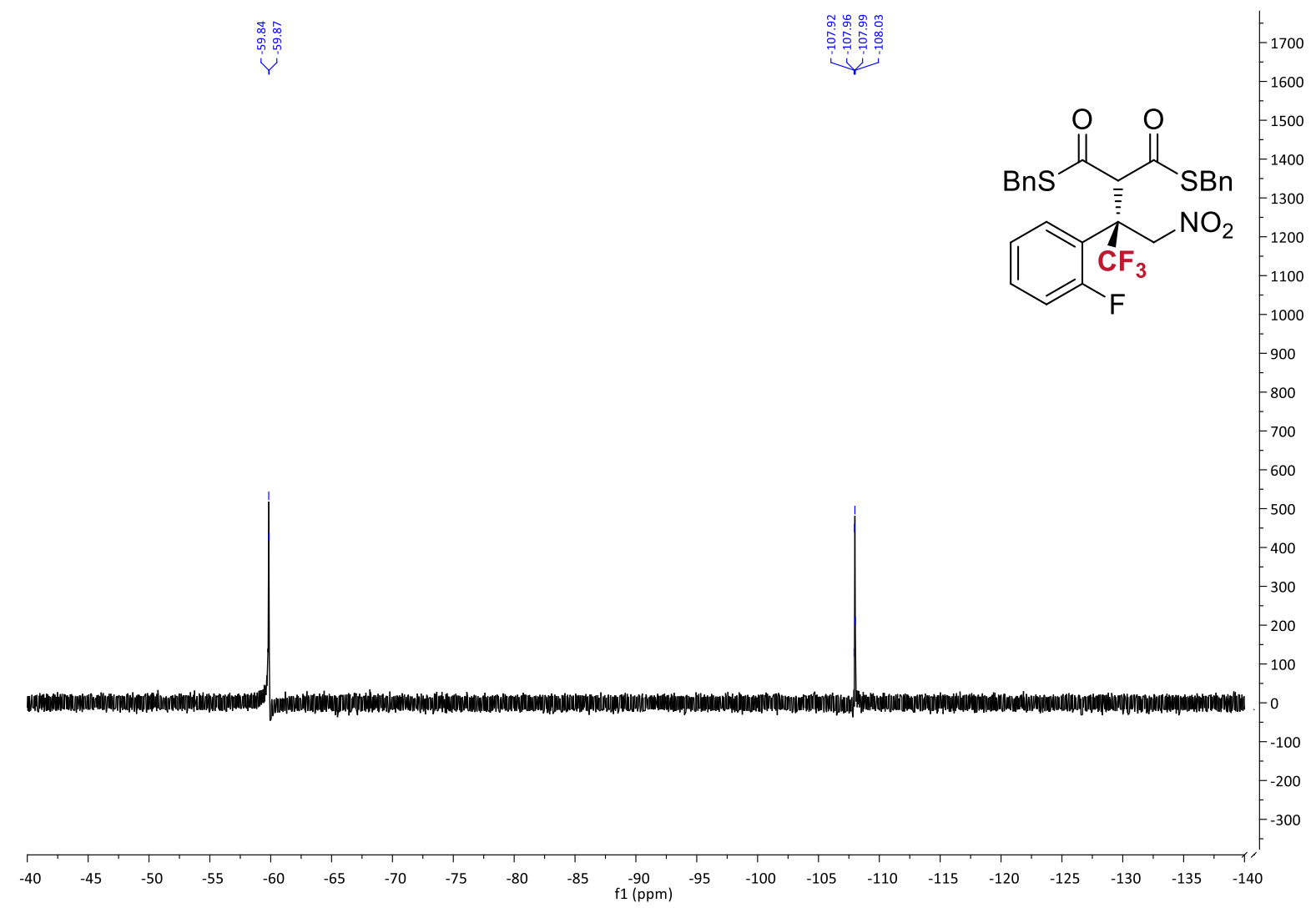

${ }^{19} \mathrm{~F}$ NMR spectrum of $\mathbf{3 c}$

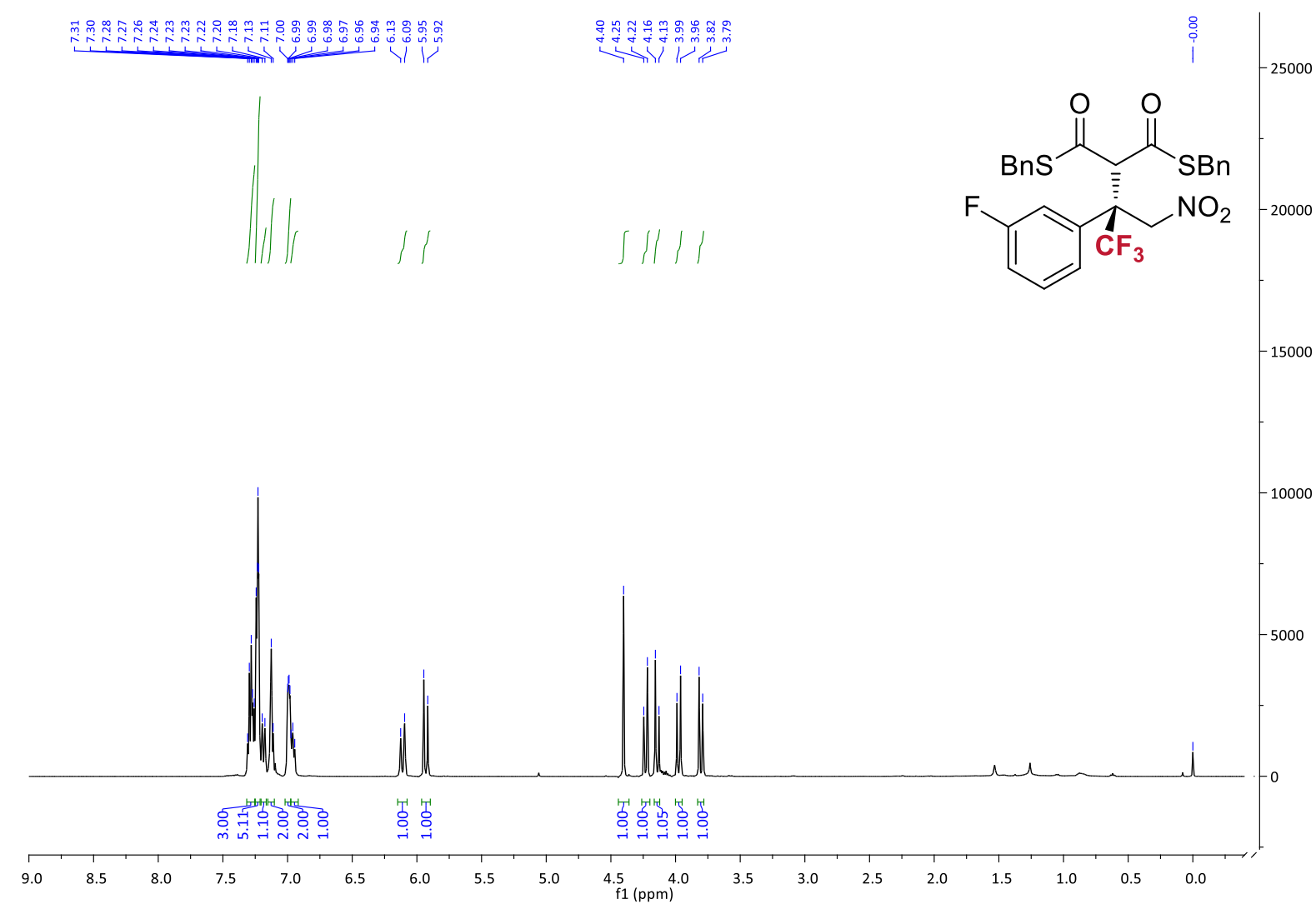

${ }^{1} \mathrm{H}$ NMR spectrum of $\mathbf{3 d}$ 


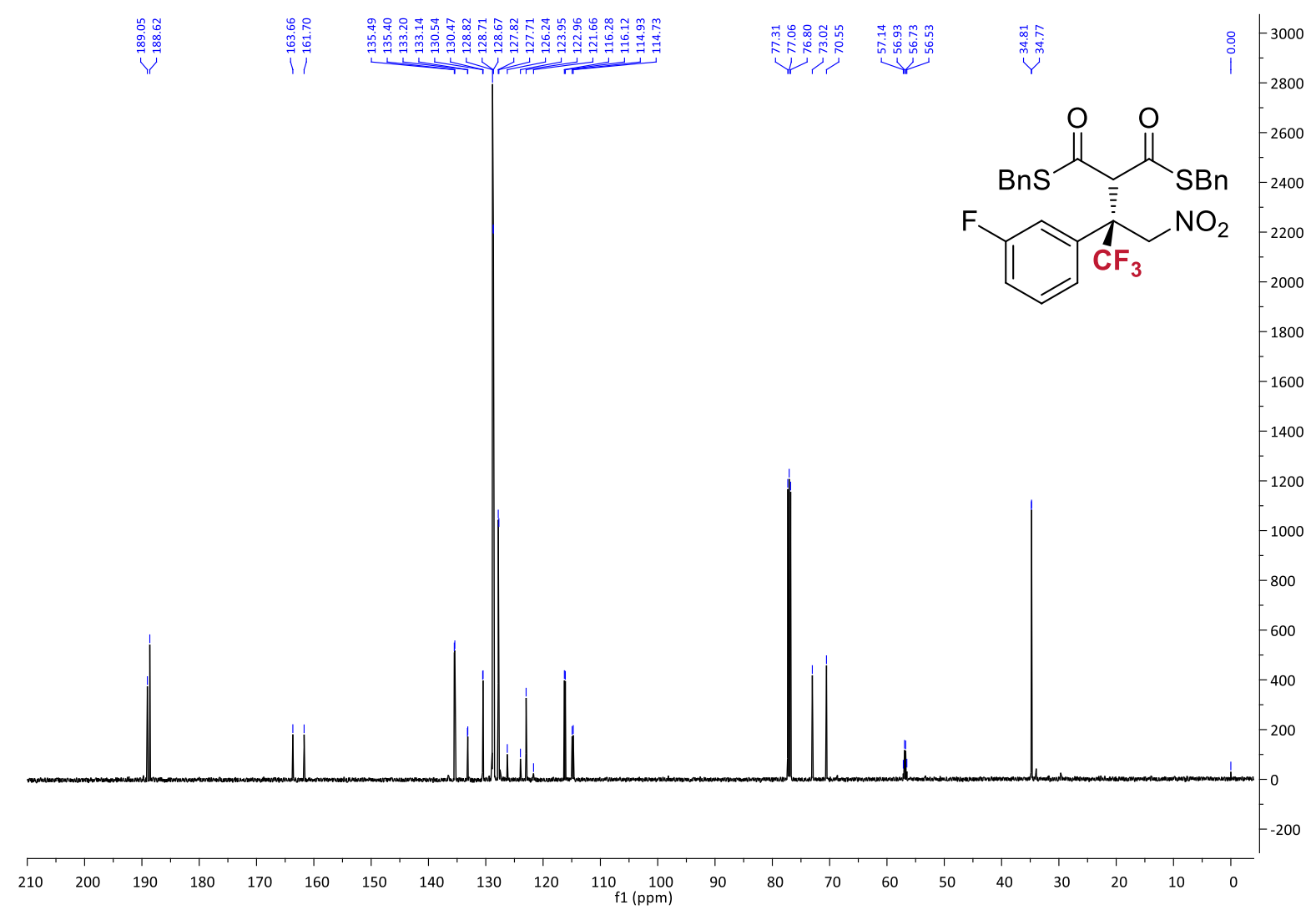

${ }^{13} \mathrm{C}$ NMR spectrum of $\mathbf{3 d}$

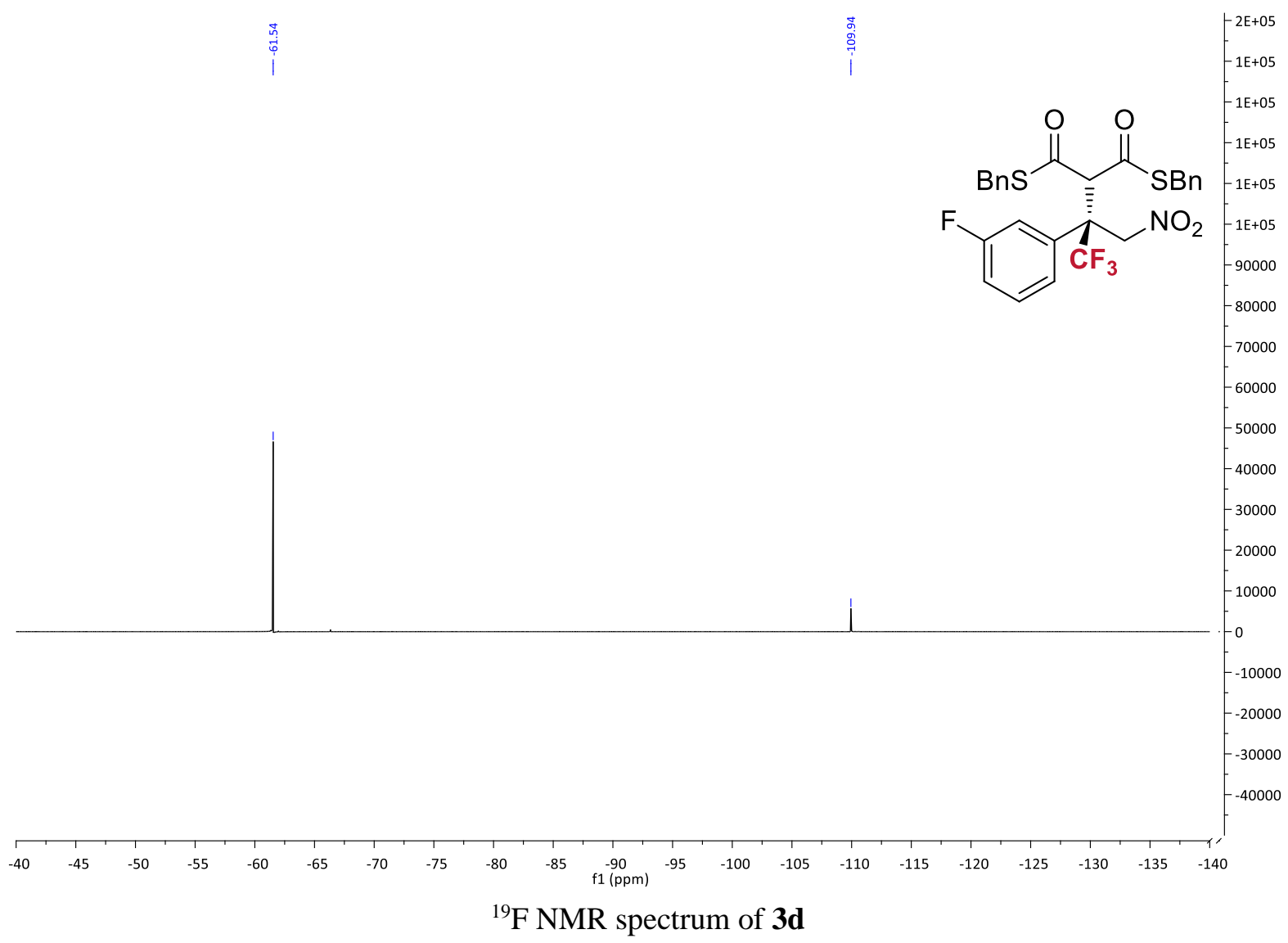




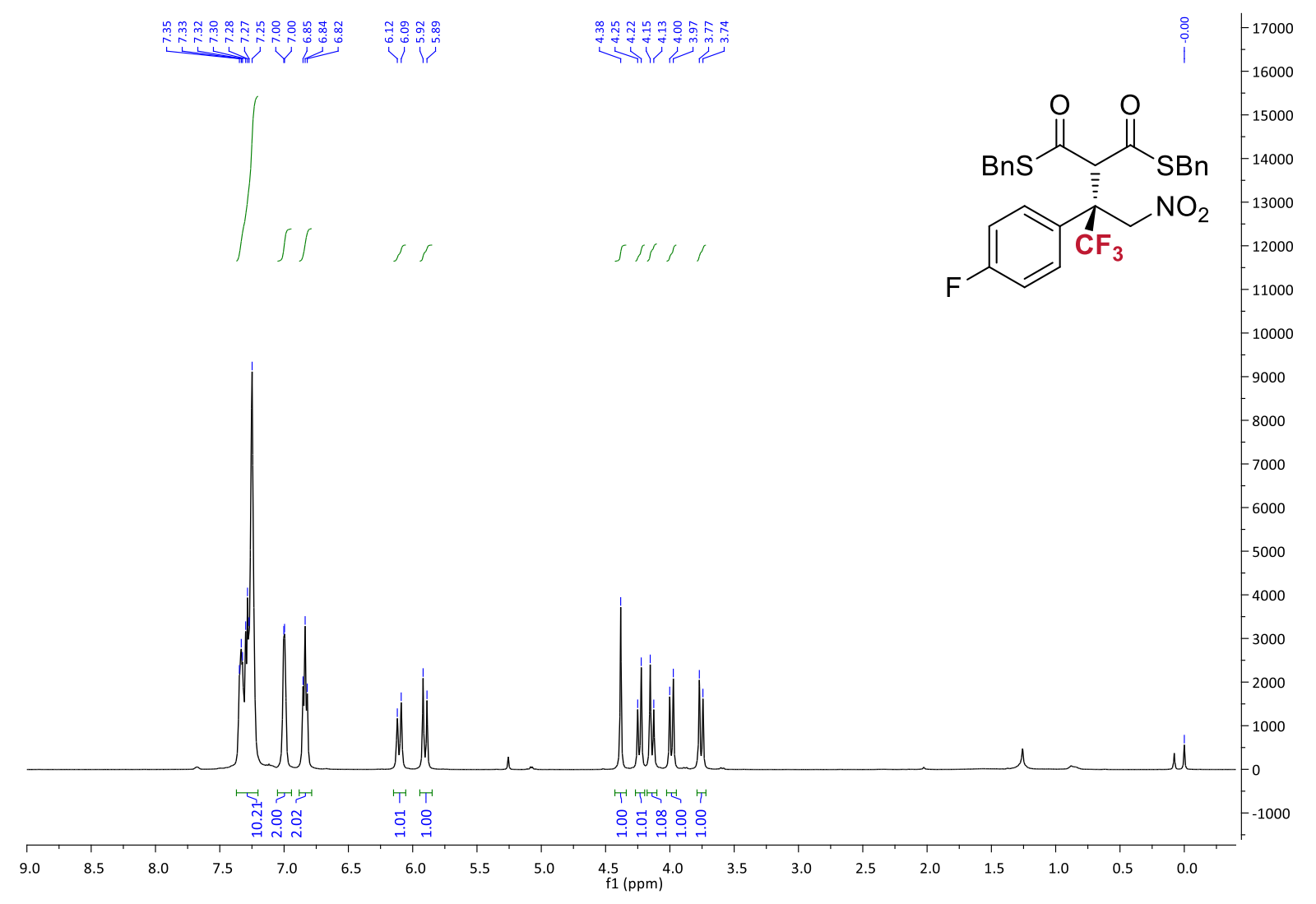

${ }^{1} \mathrm{H}$ NMR spectrum of $\mathbf{3 e}$

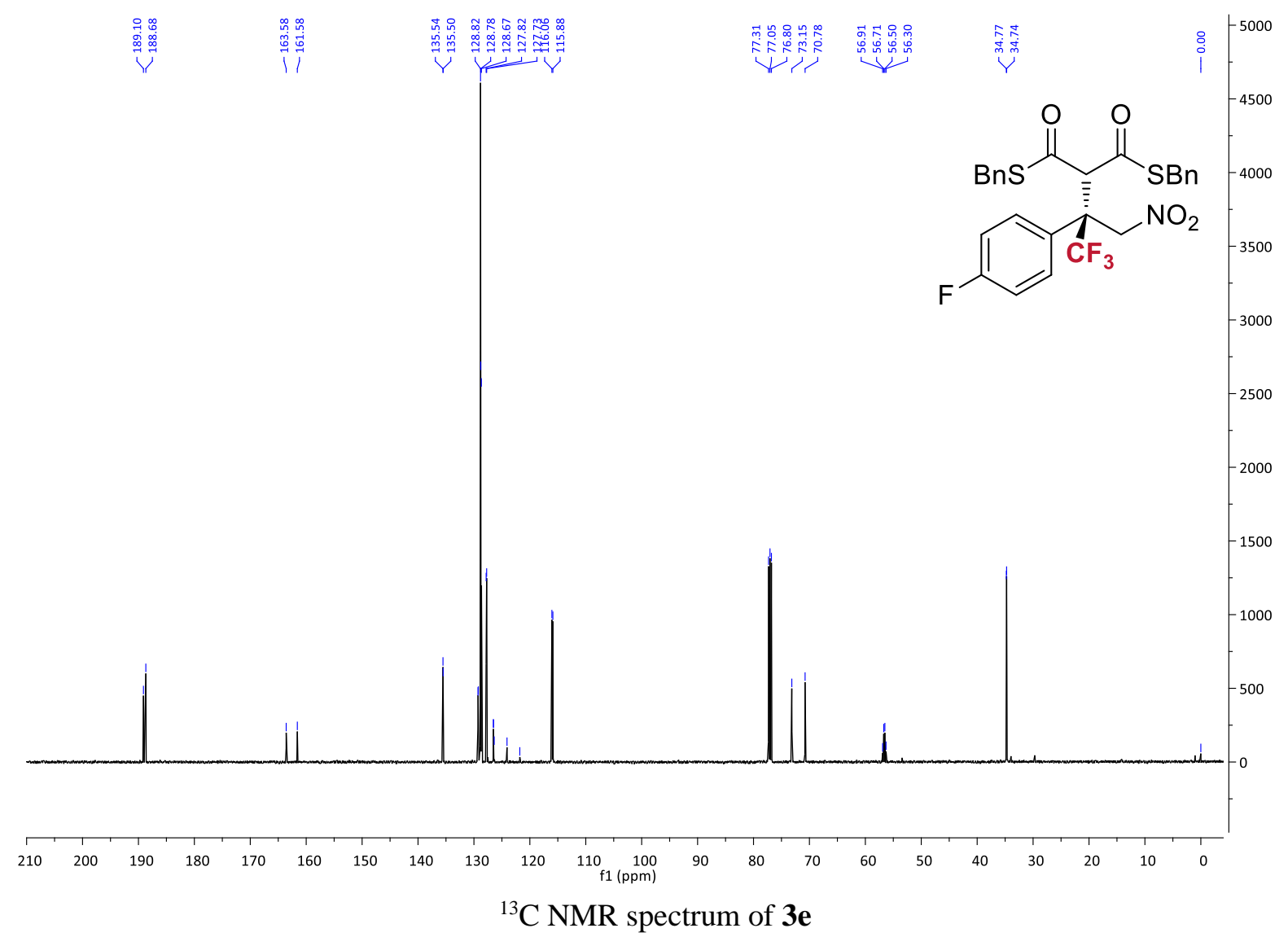




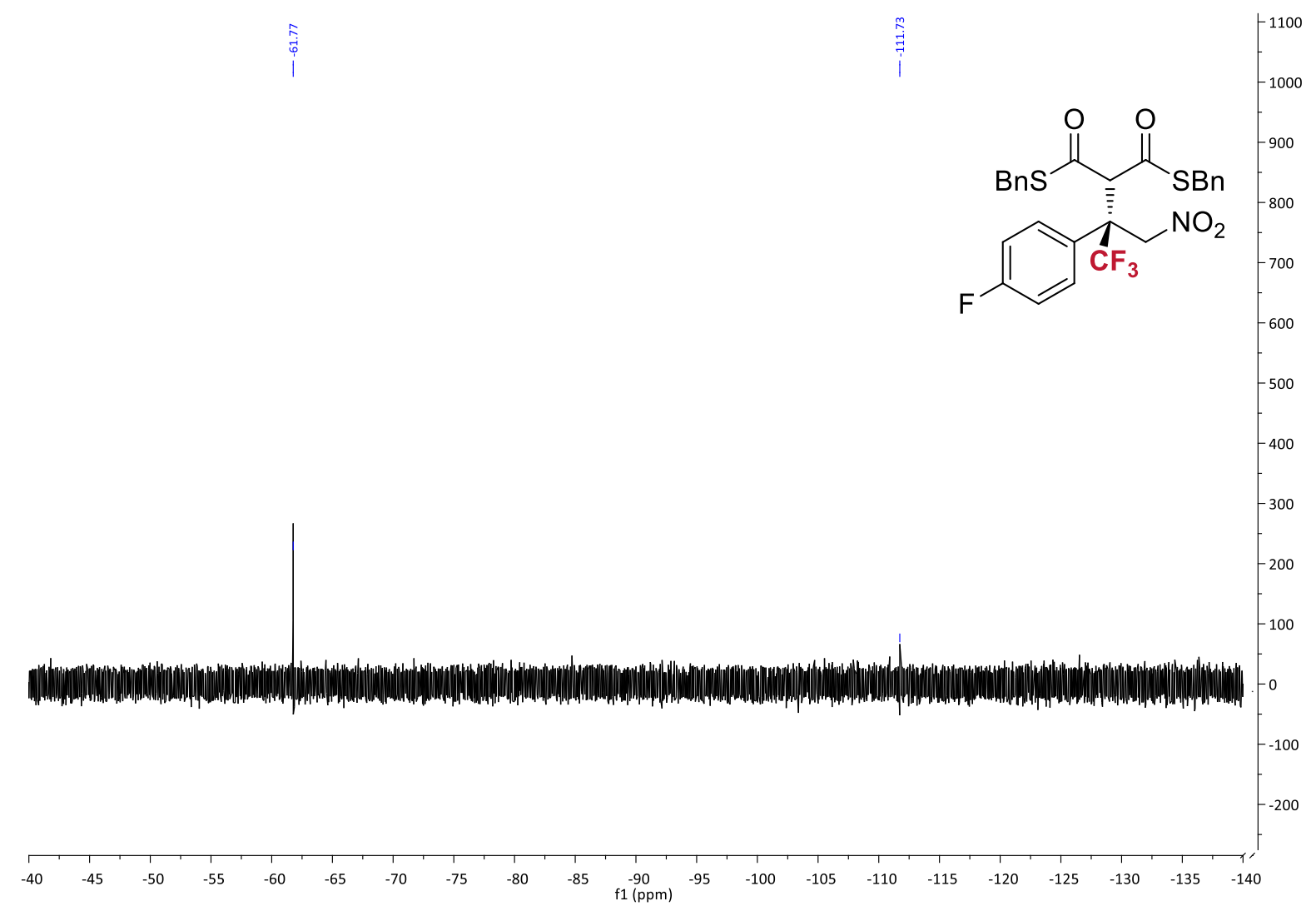

${ }^{19} \mathrm{~F}$ NMR spectrum of $\mathbf{3 e}$

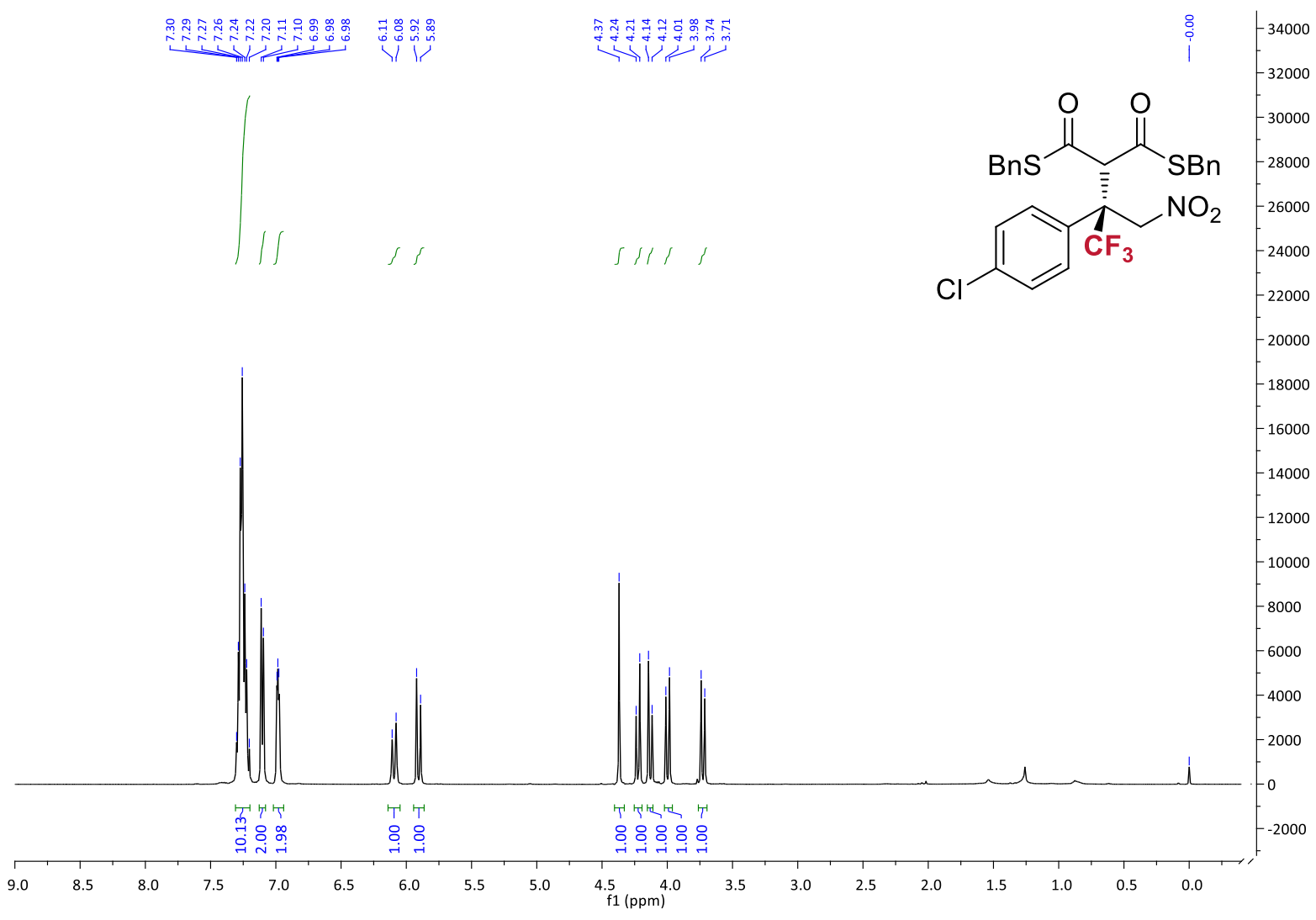

${ }^{1} \mathrm{H}$ NMR spectrum of $\mathbf{3 f}$ 


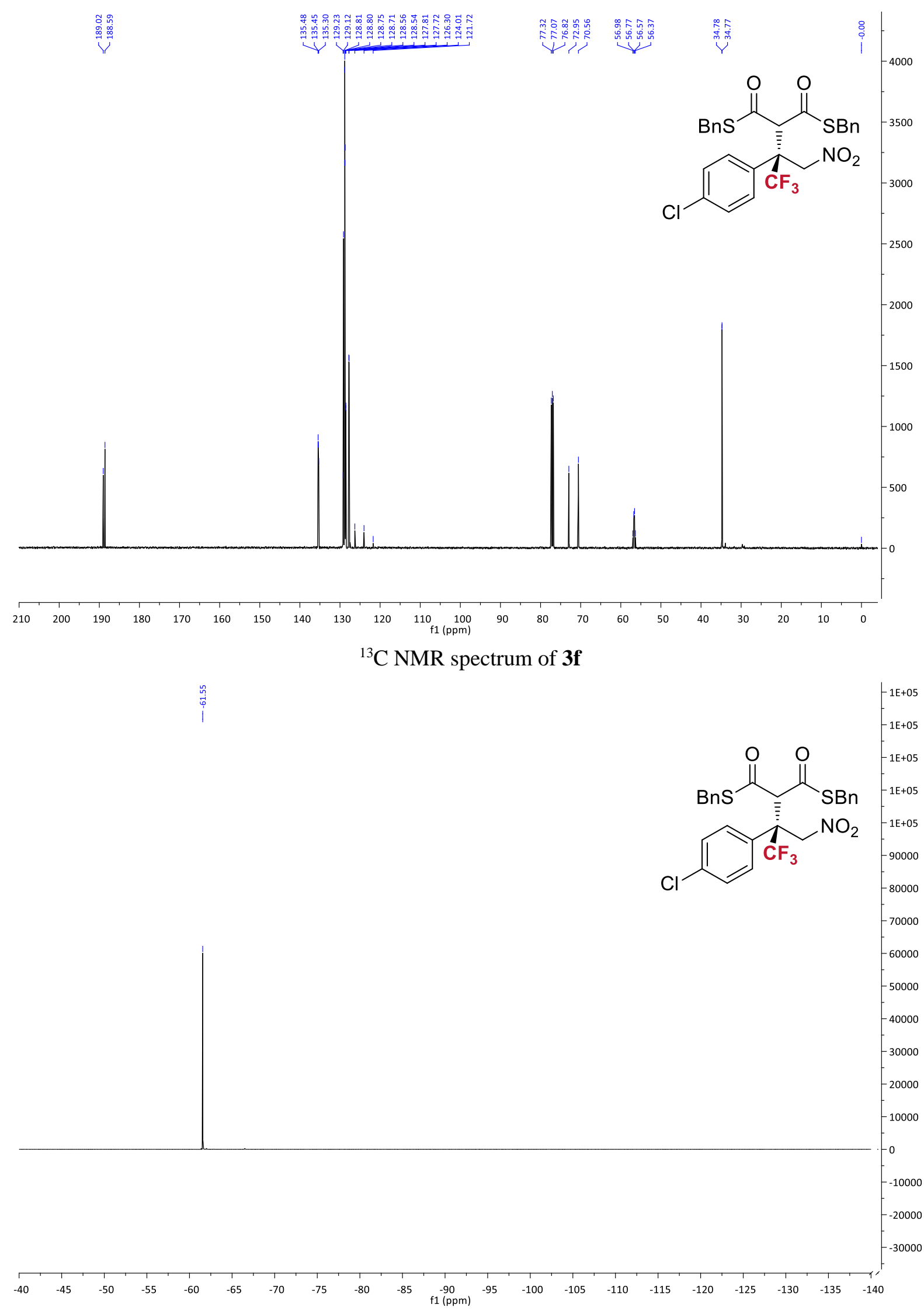

${ }^{19} \mathrm{~F}$ NMR spectrum of $\mathbf{3 f}$ 


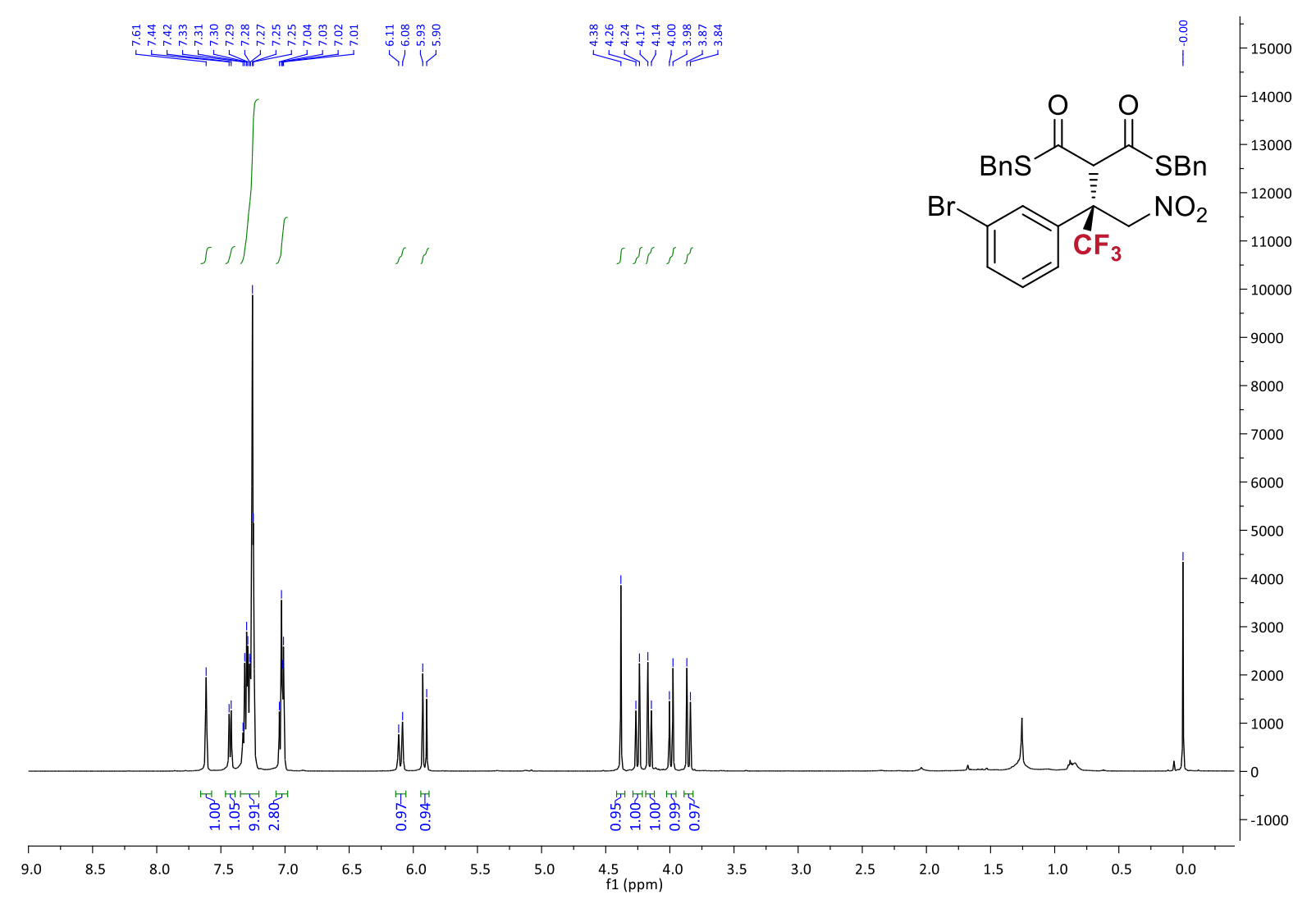

${ }^{1} \mathrm{H}$ NMR spectrum of $\mathbf{3 g}$

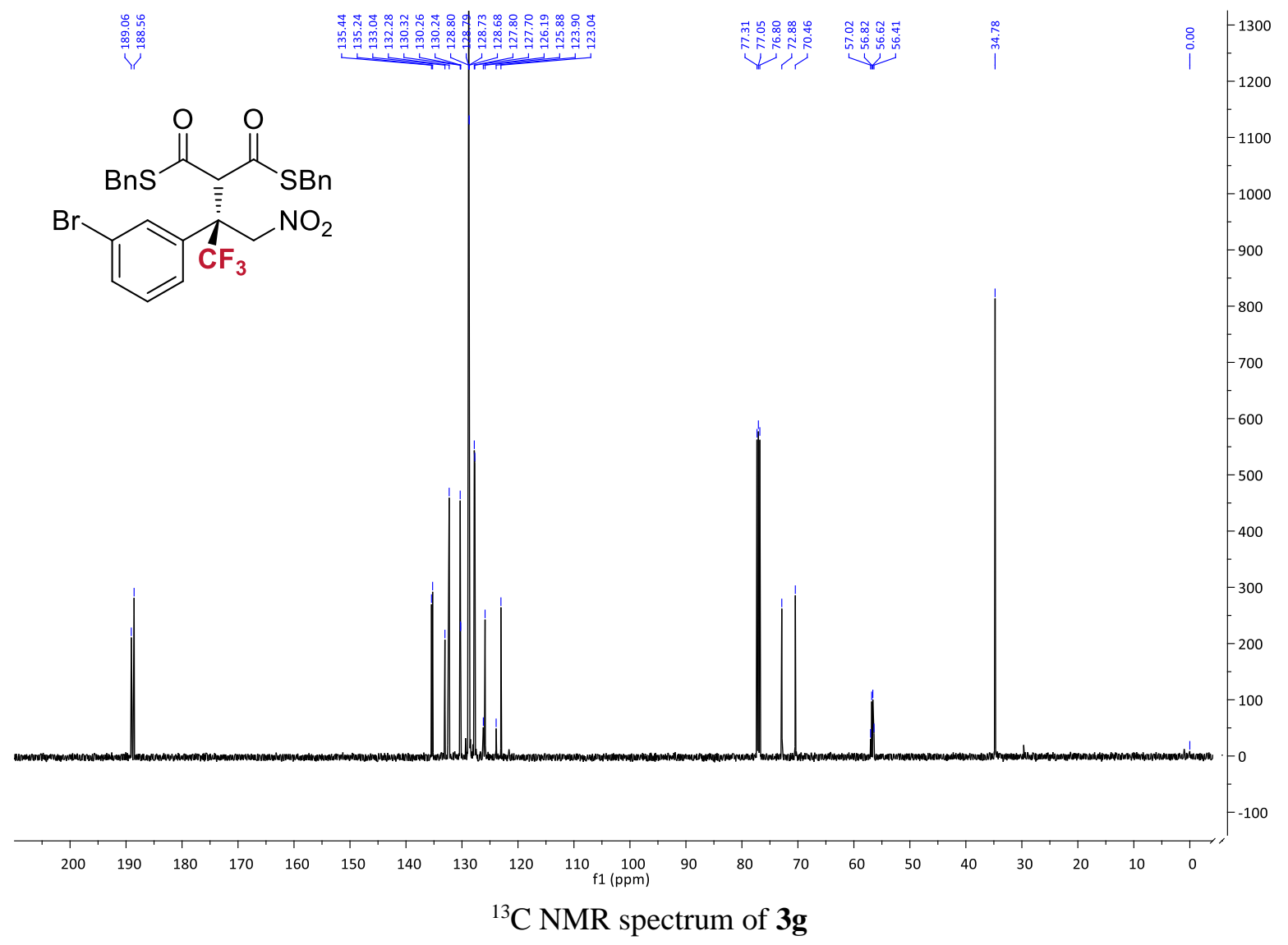




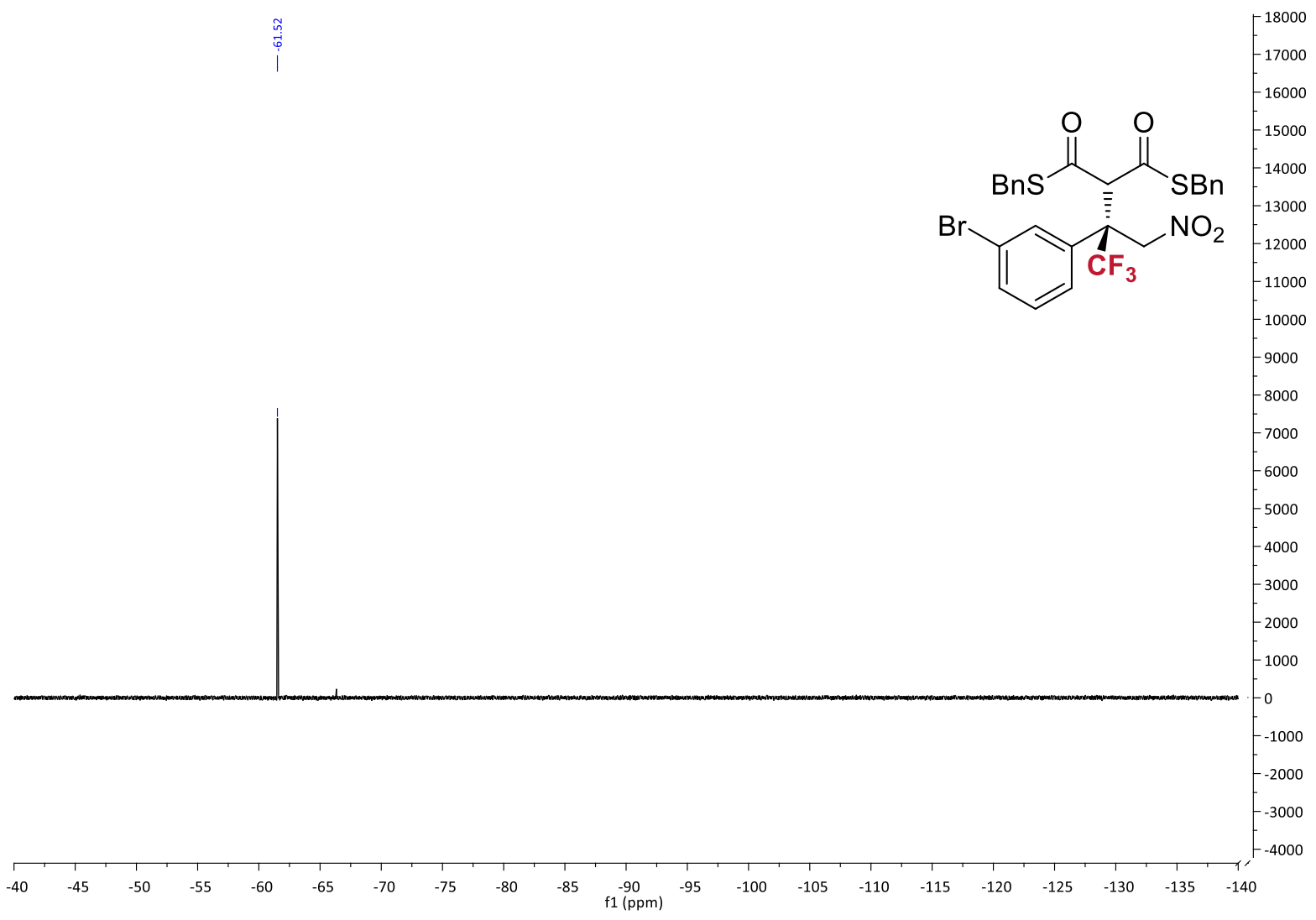

${ }^{19} \mathrm{~F}$ NMR spectrum of $\mathbf{3 g}$

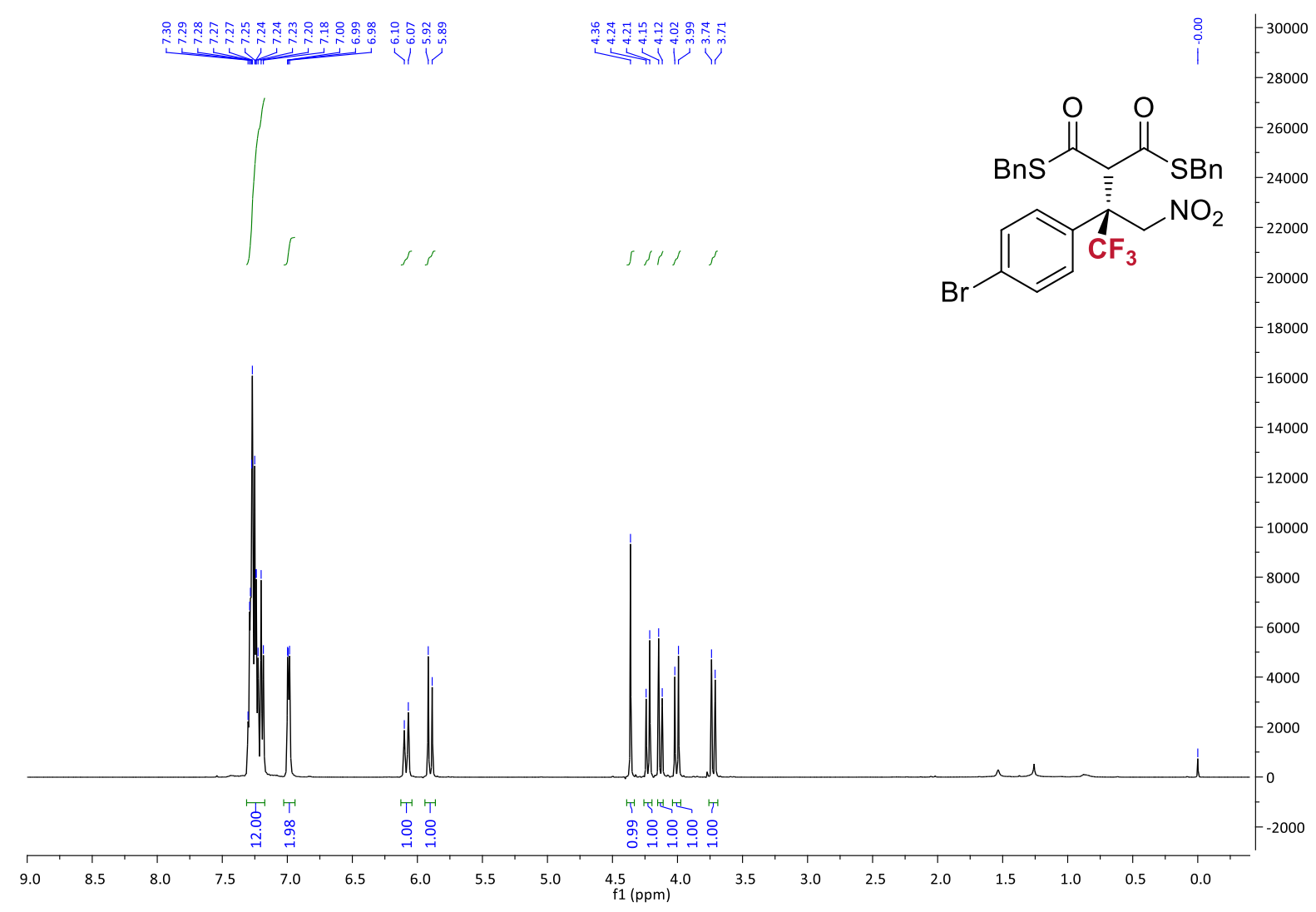

${ }^{1} \mathrm{H}$ NMR spectrum of $\mathbf{3 h}$ 


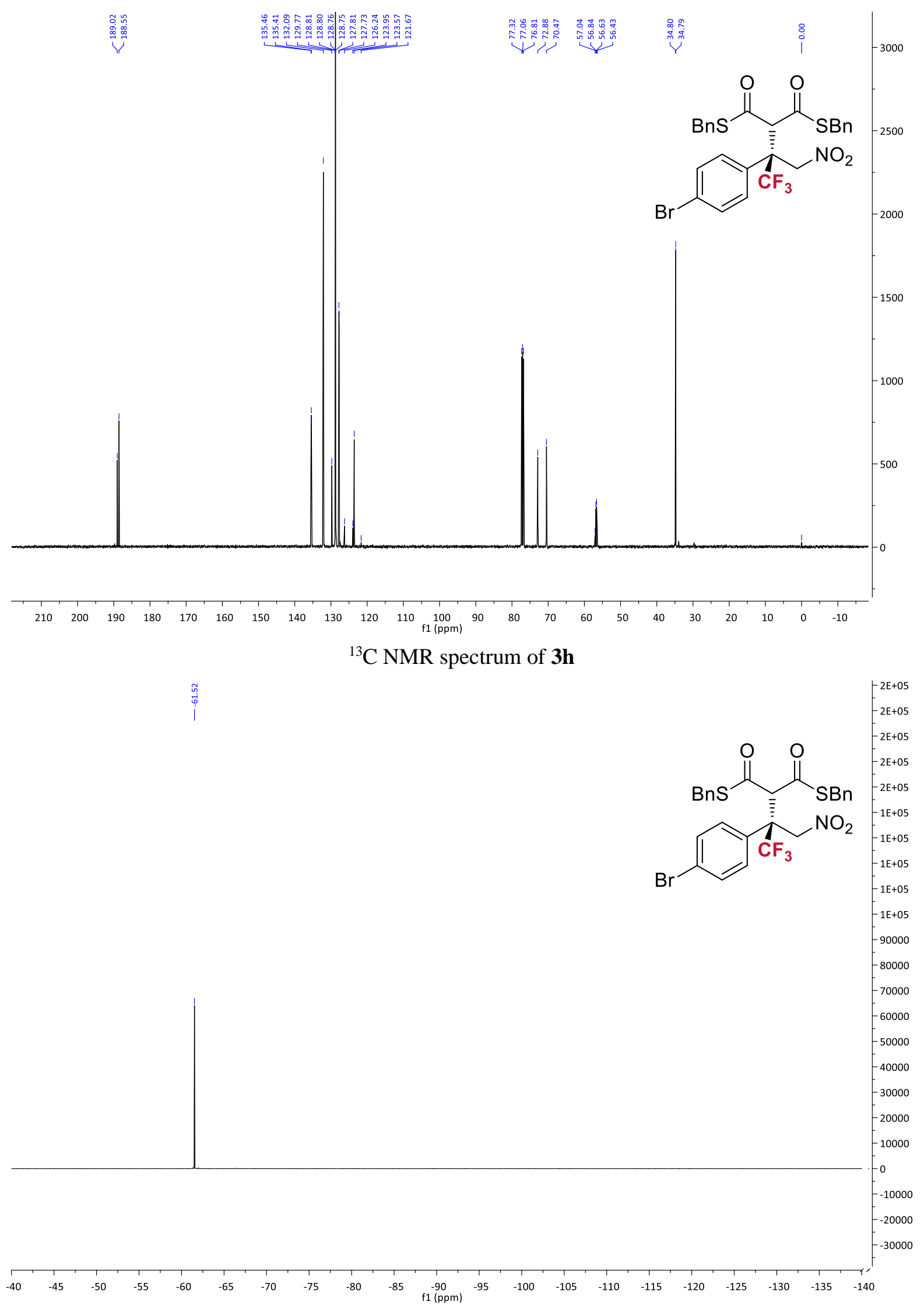

${ }^{19} \mathrm{~F}$ NMR spectrum of $\mathbf{3 h}$ 


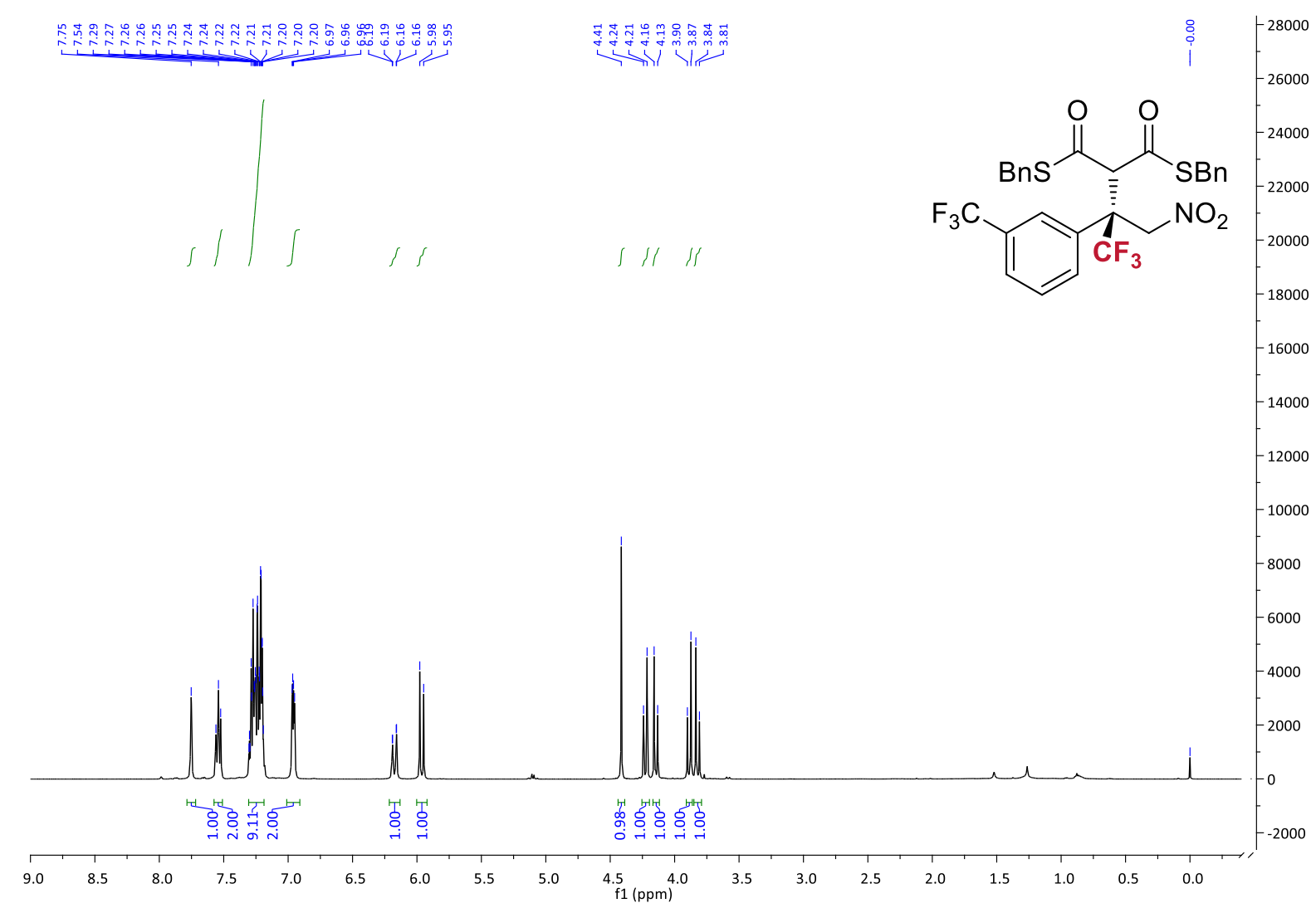

${ }^{1} \mathrm{H}$ NMR spectrum of $\mathbf{3 i}$

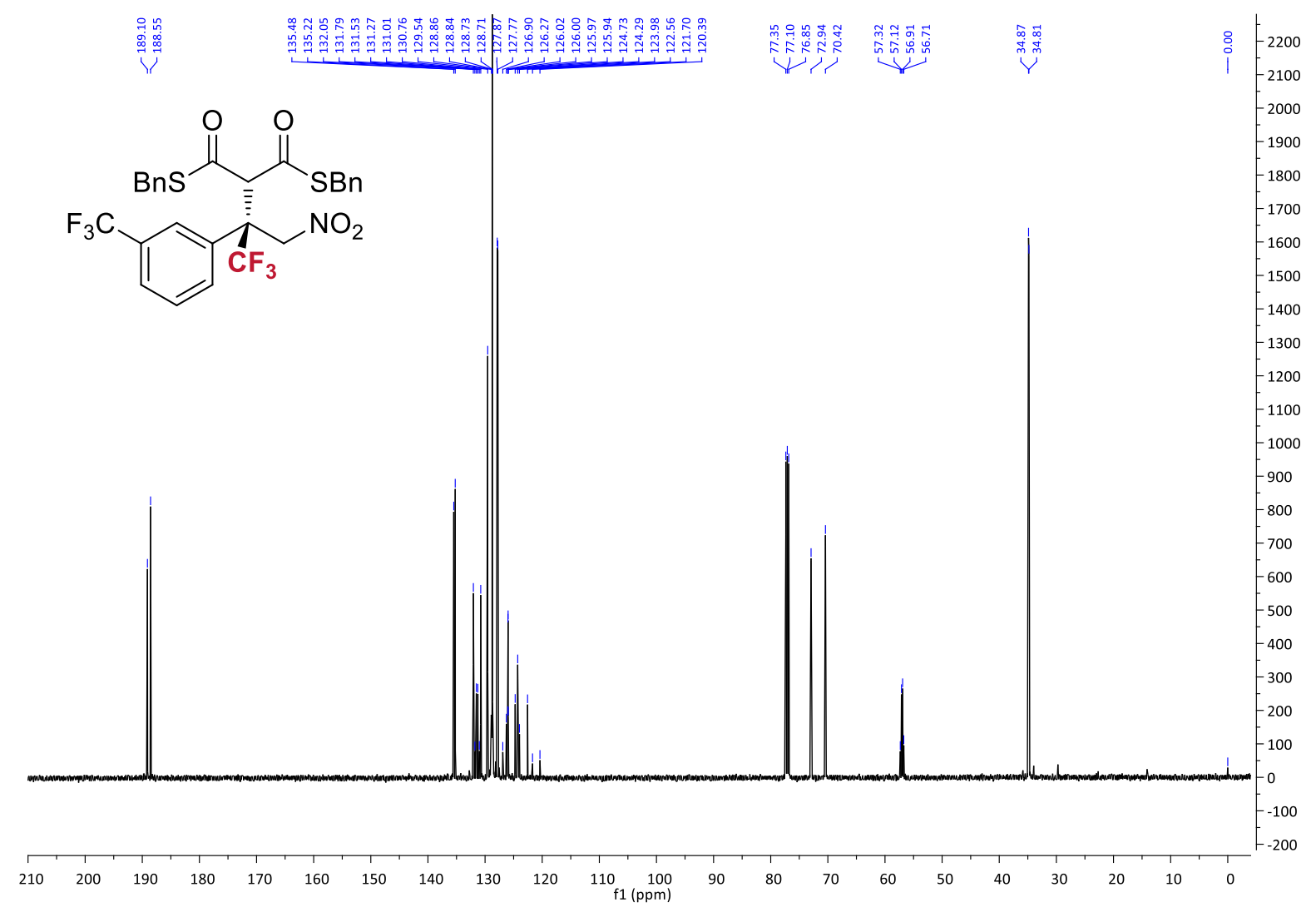

${ }^{13} \mathrm{C}$ NMR spectrum of $\mathbf{3 i}$ 


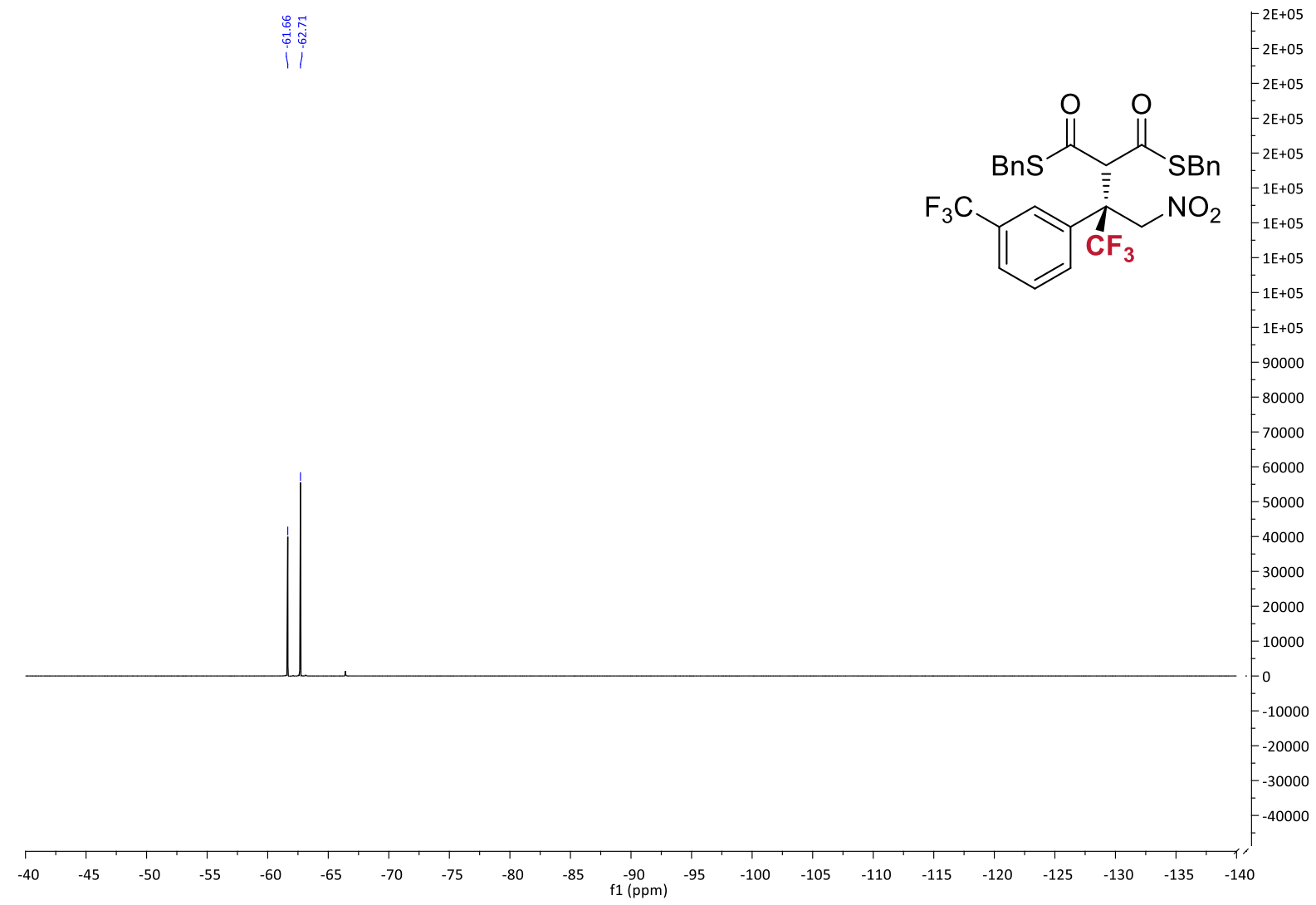

${ }^{19} \mathrm{~F}$ NMR spectrum of $\mathbf{3 i}$

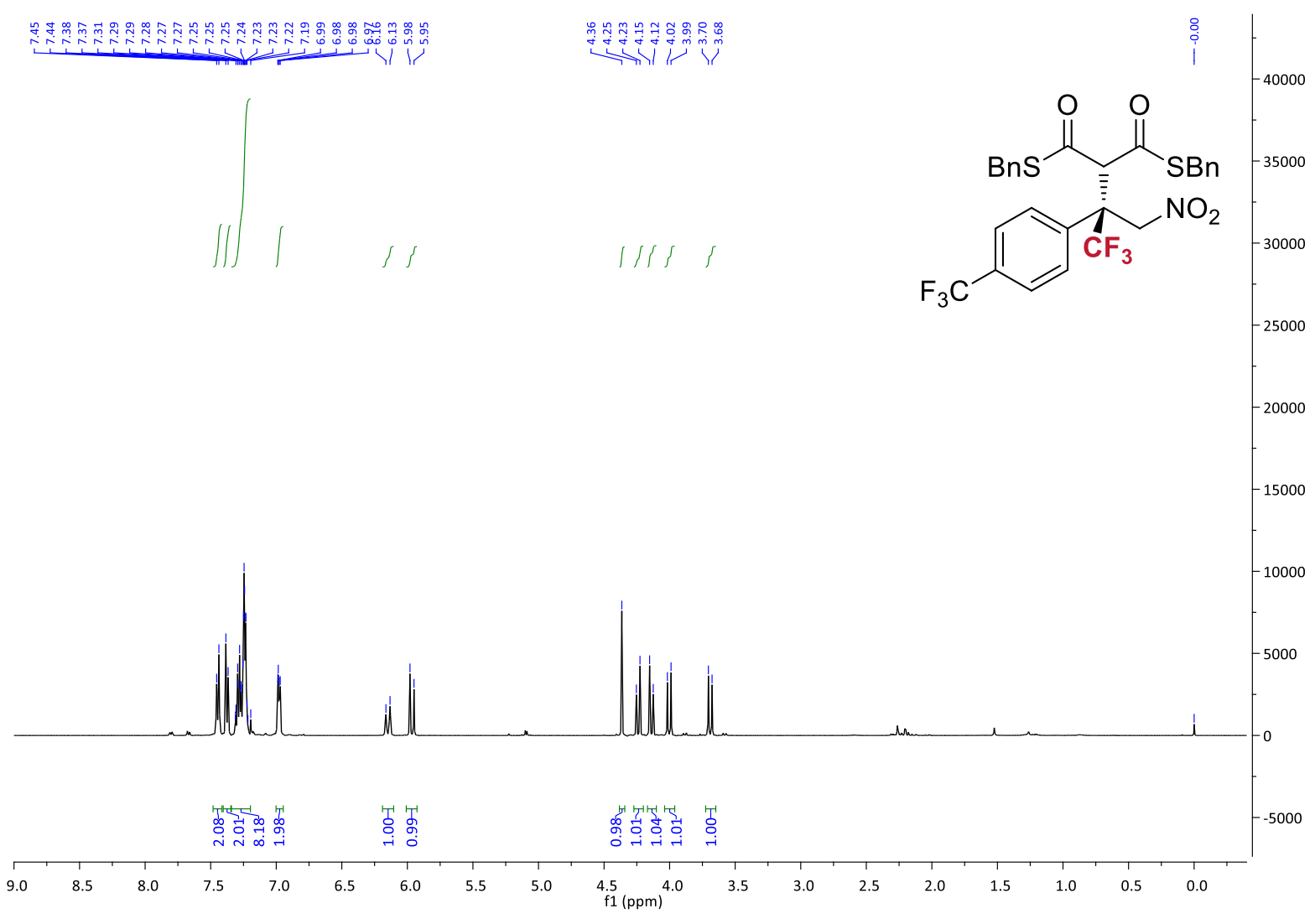

${ }^{1} \mathrm{H}$ NMR spectrum of $\mathbf{3 j}$ 


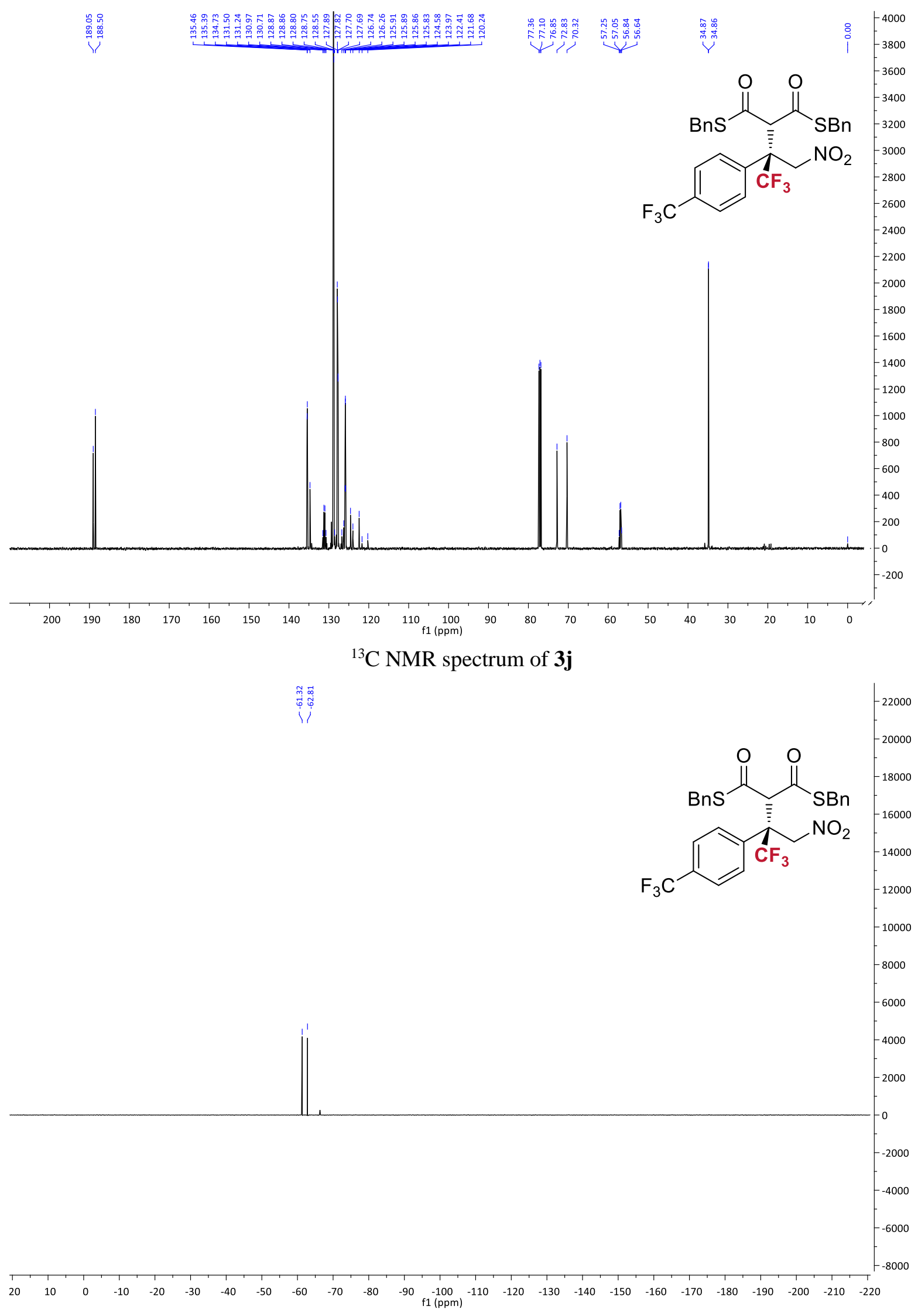

${ }^{19} \mathrm{~F}$ NMR spectrum of $\mathbf{3 j}$ 


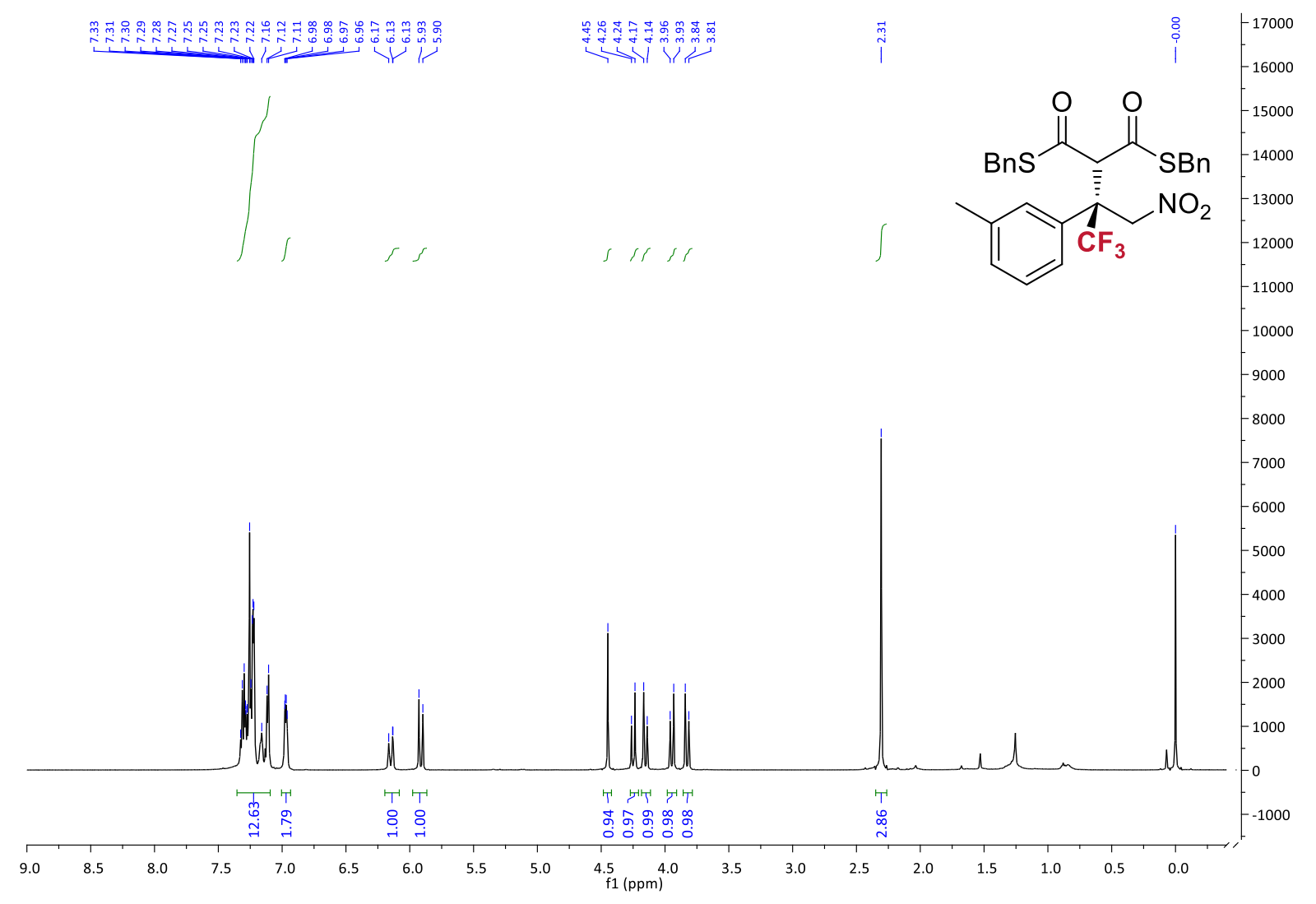

${ }^{1} \mathrm{H}$ NMR spectrum of $\mathbf{3 k}$

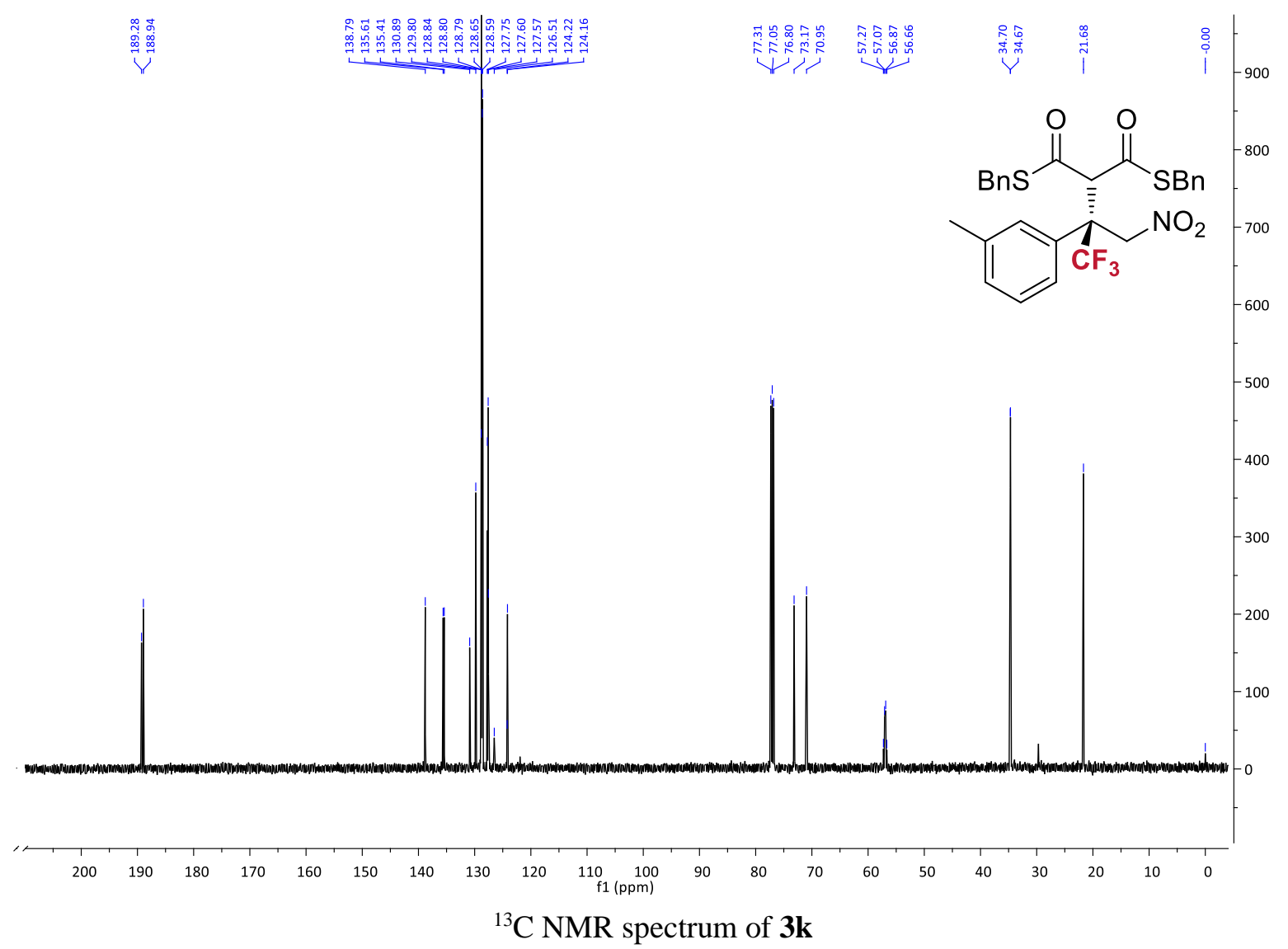




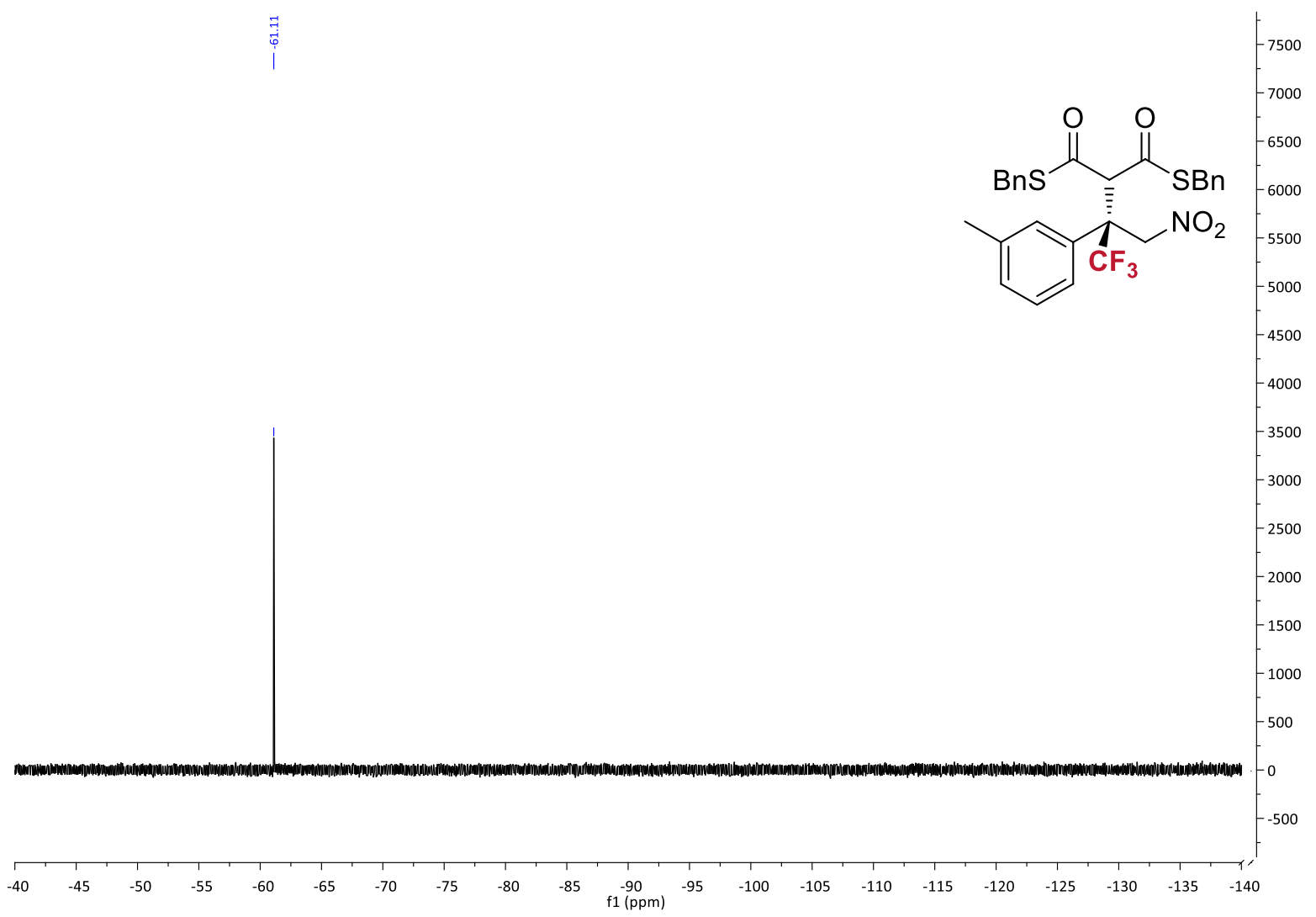

${ }^{19} \mathrm{~F}$ NMR spectrum of $\mathbf{3 k}$

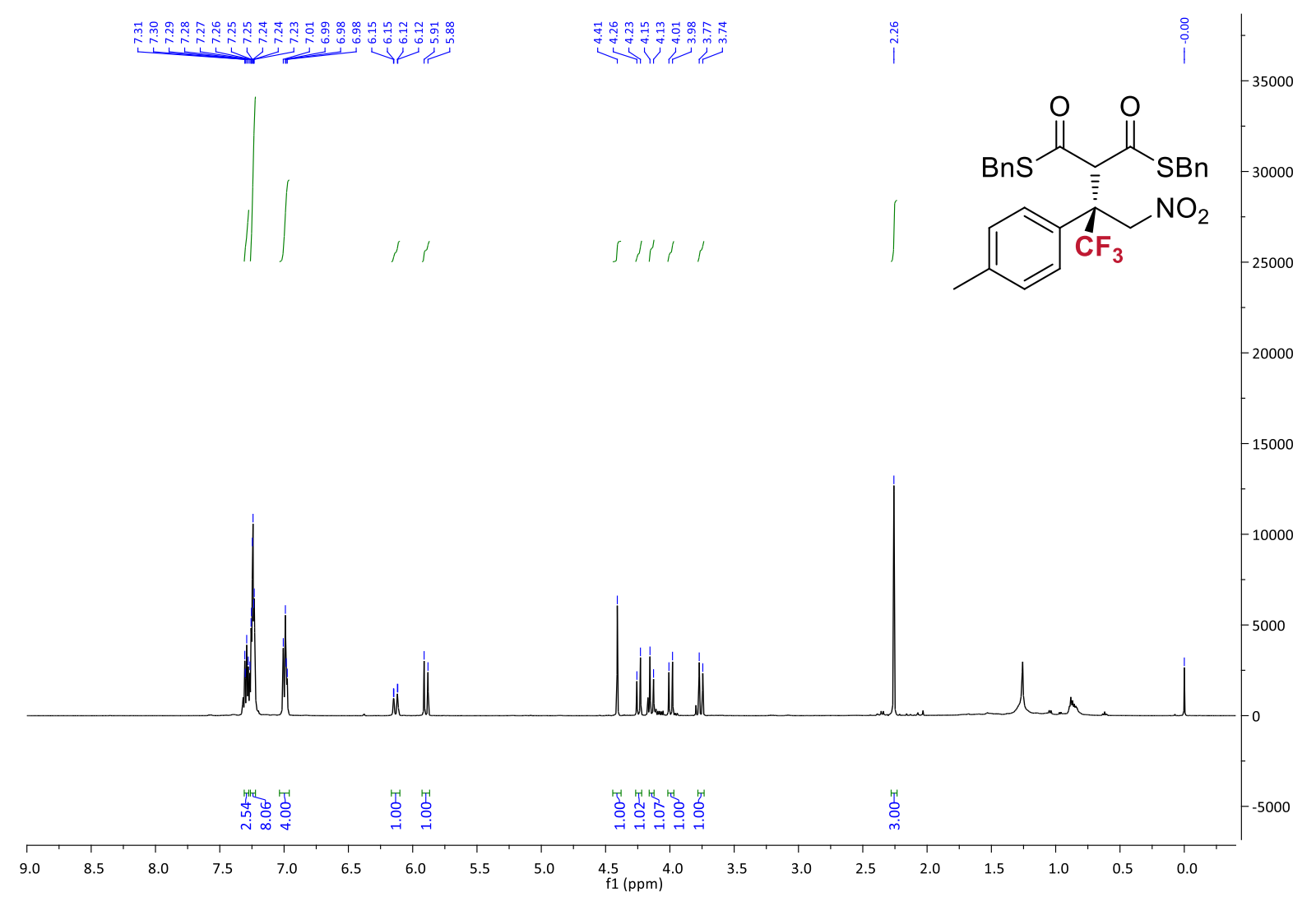

${ }^{1} \mathrm{H}$ NMR spectrum of $\mathbf{3 l}$ 


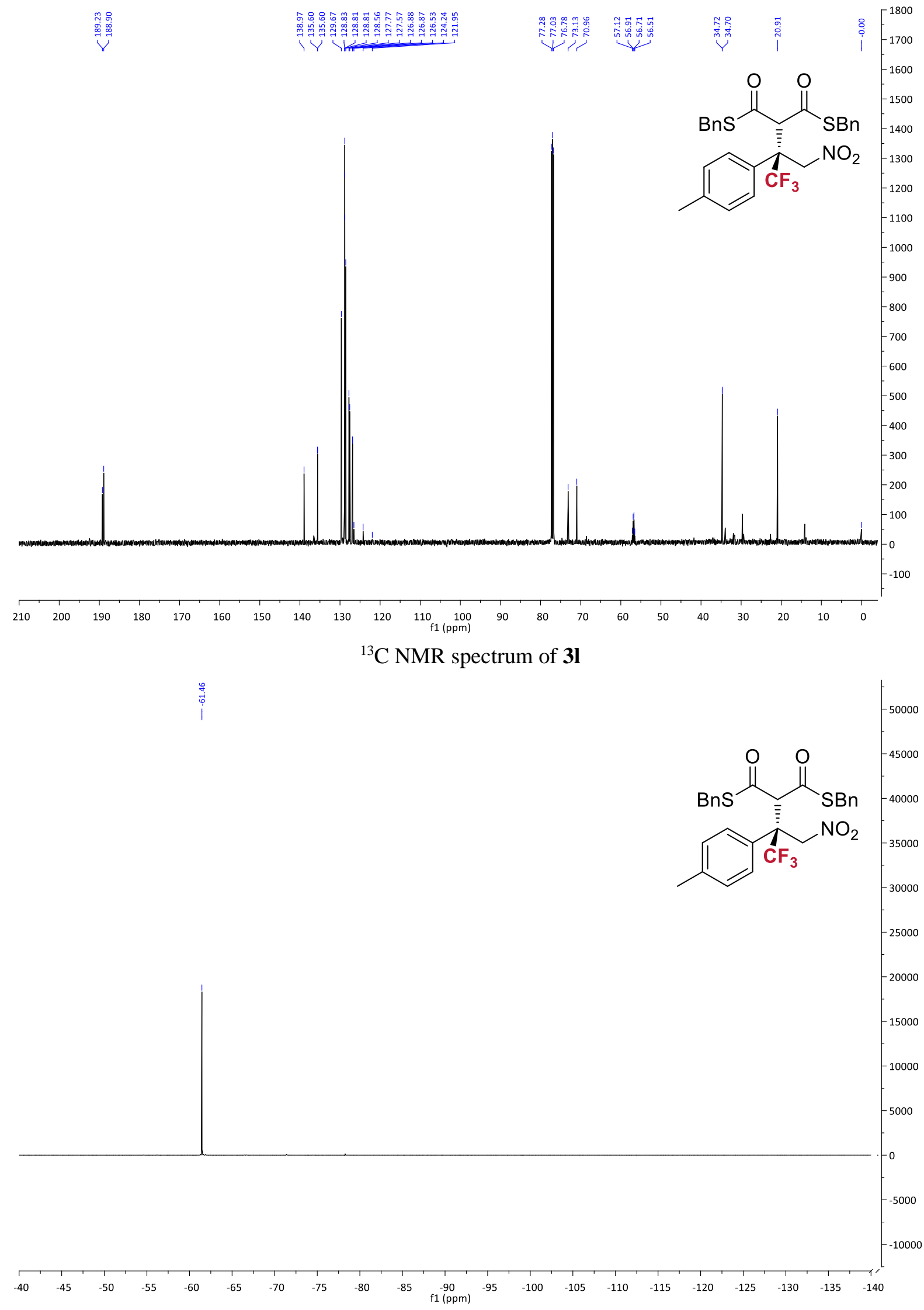

${ }^{19} \mathrm{~F}$ NMR spectrum of $\mathbf{3 l}$ 


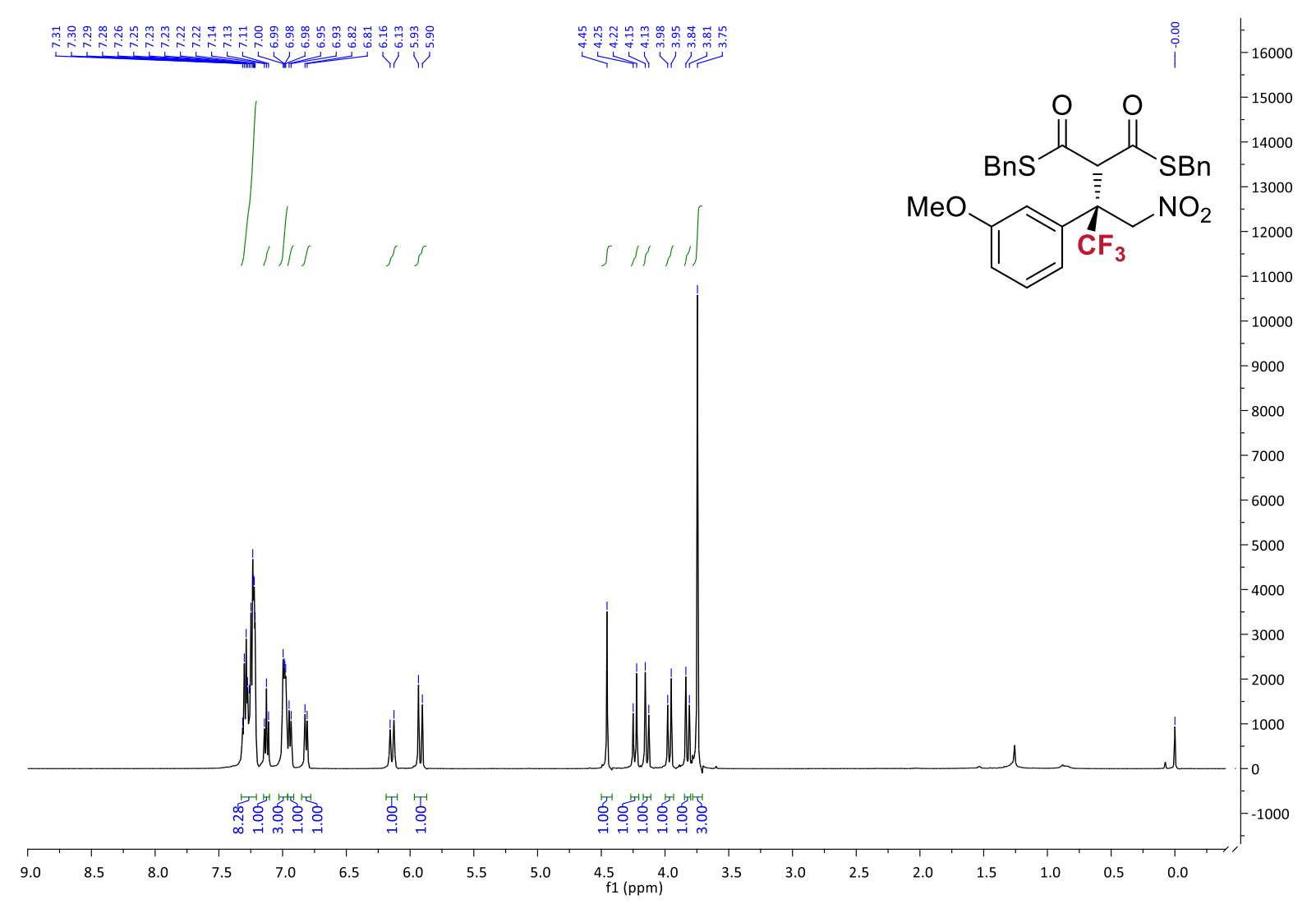

${ }^{1} \mathrm{H}$ NMR spectrum of $\mathbf{3 m}$

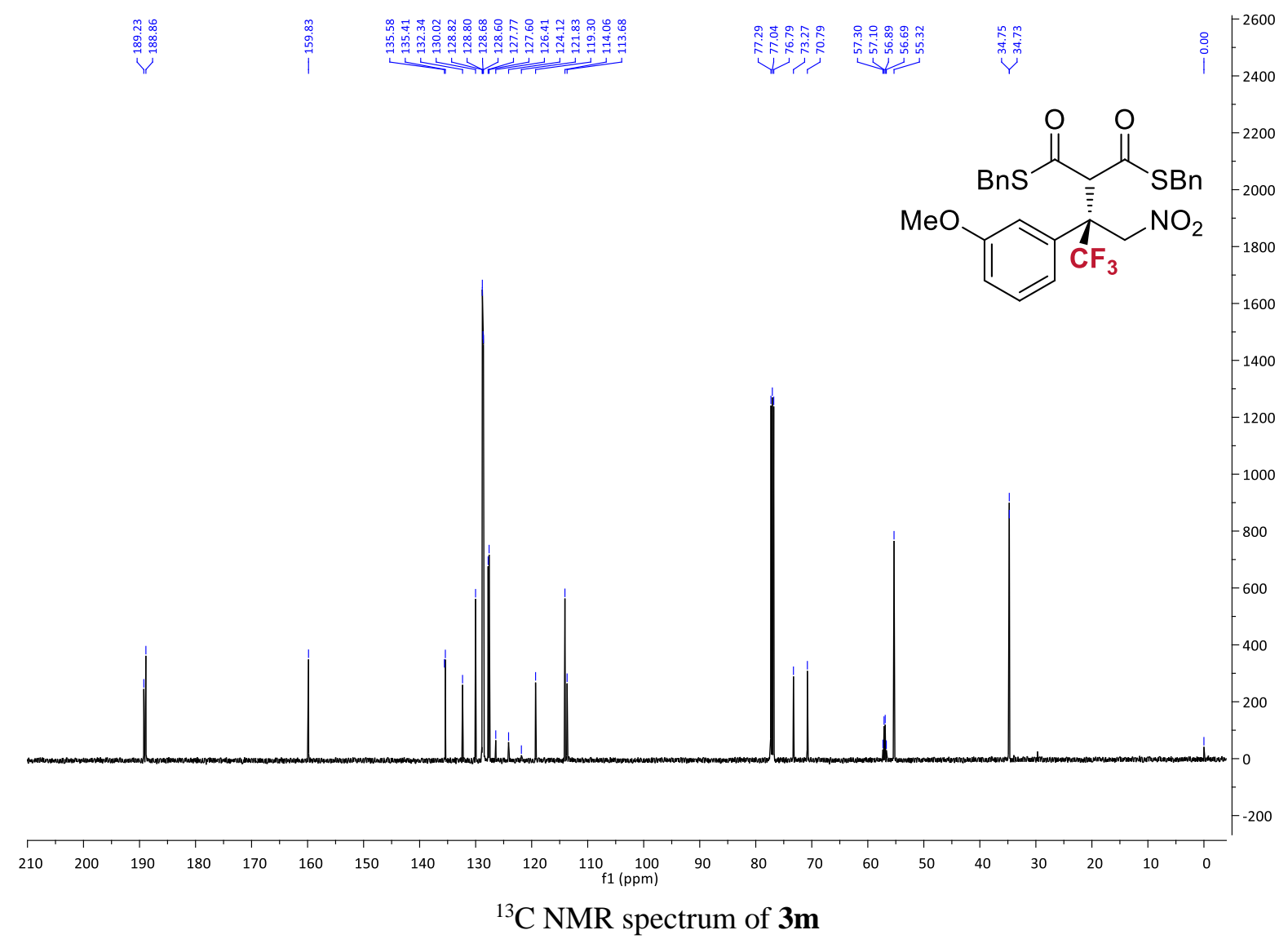




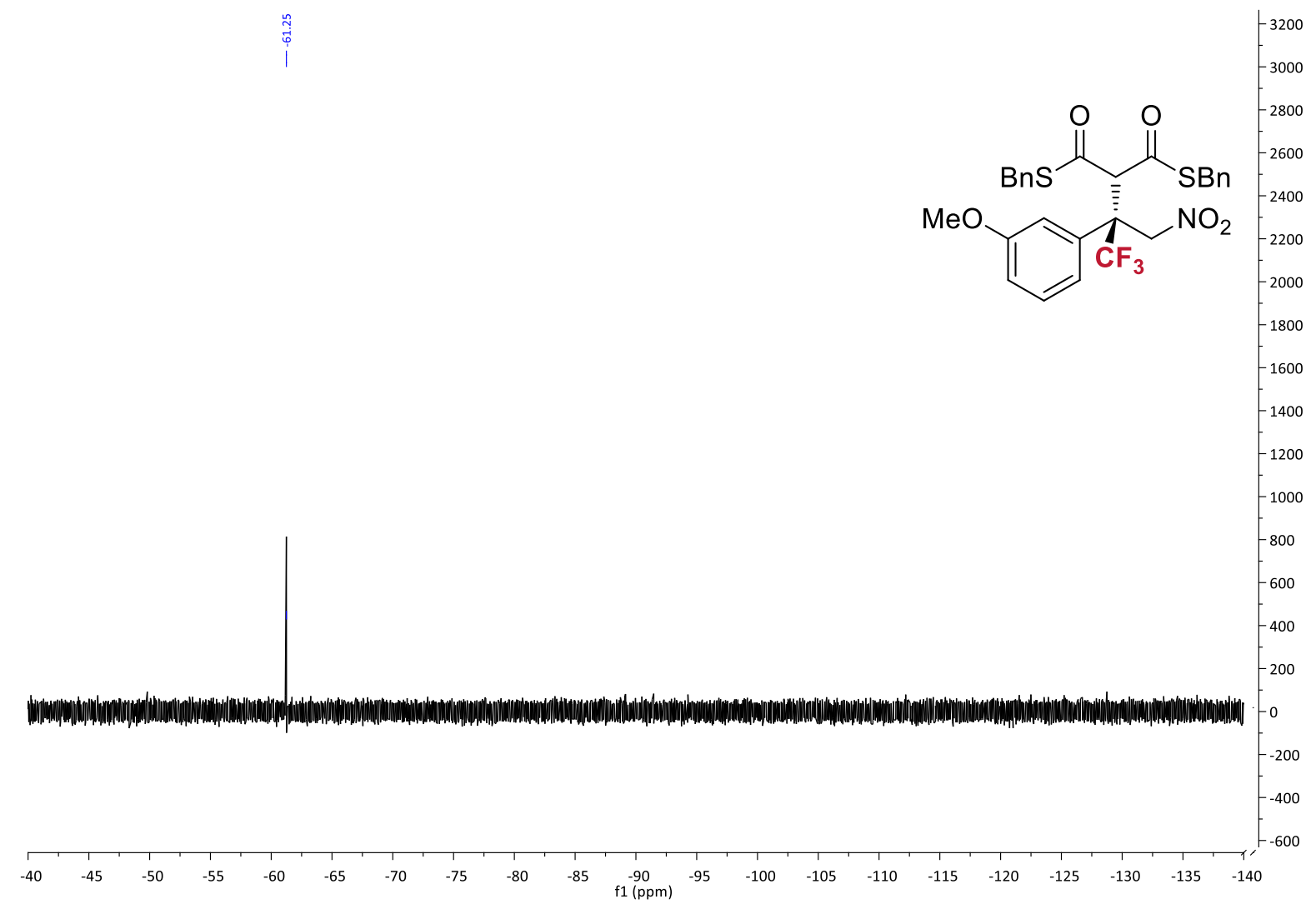

${ }^{19} \mathrm{~F}$ NMR spectrum of $\mathbf{3 m}$

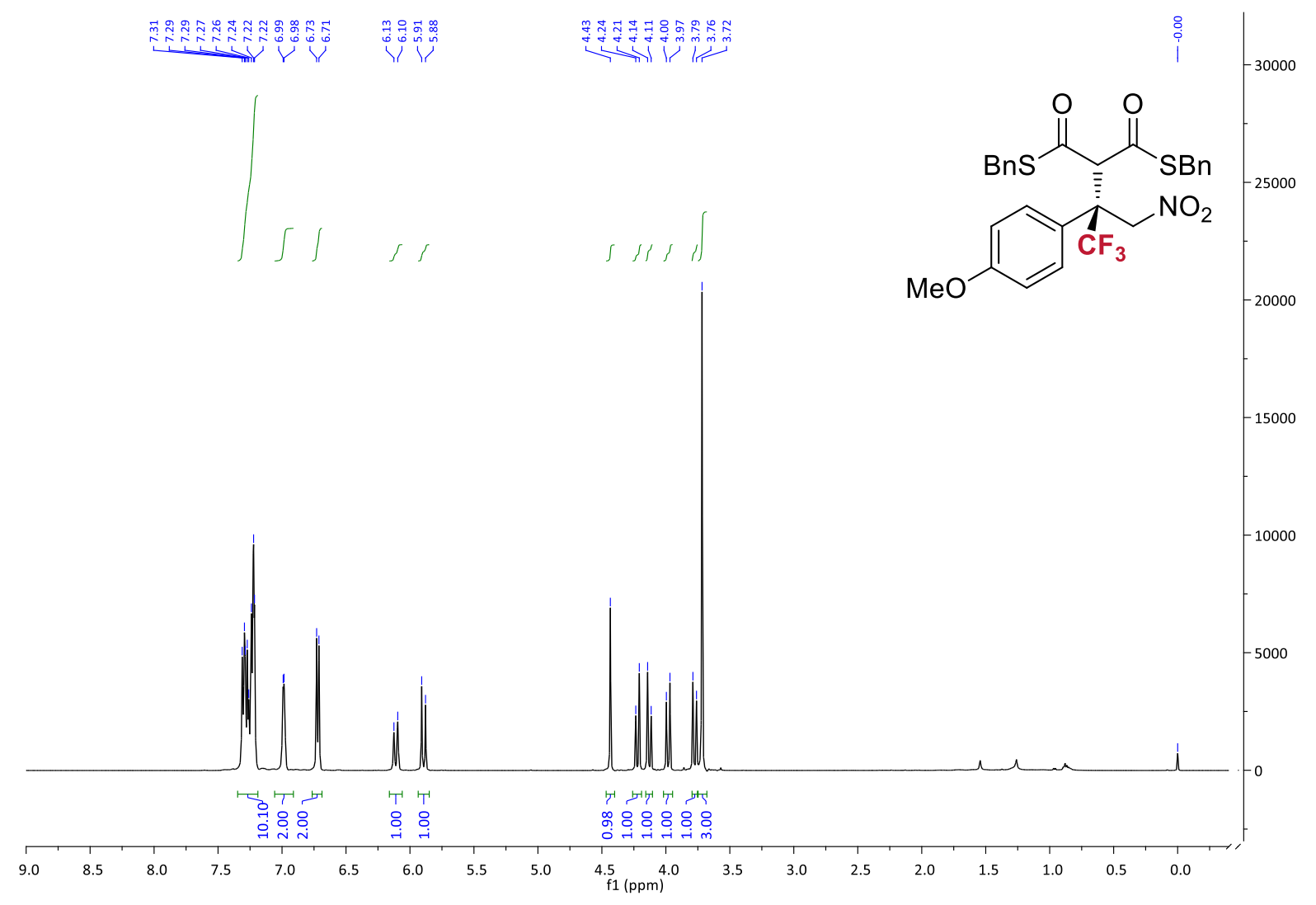

${ }^{1} \mathrm{H}$ NMR spectrum of $\mathbf{3 n}$ 


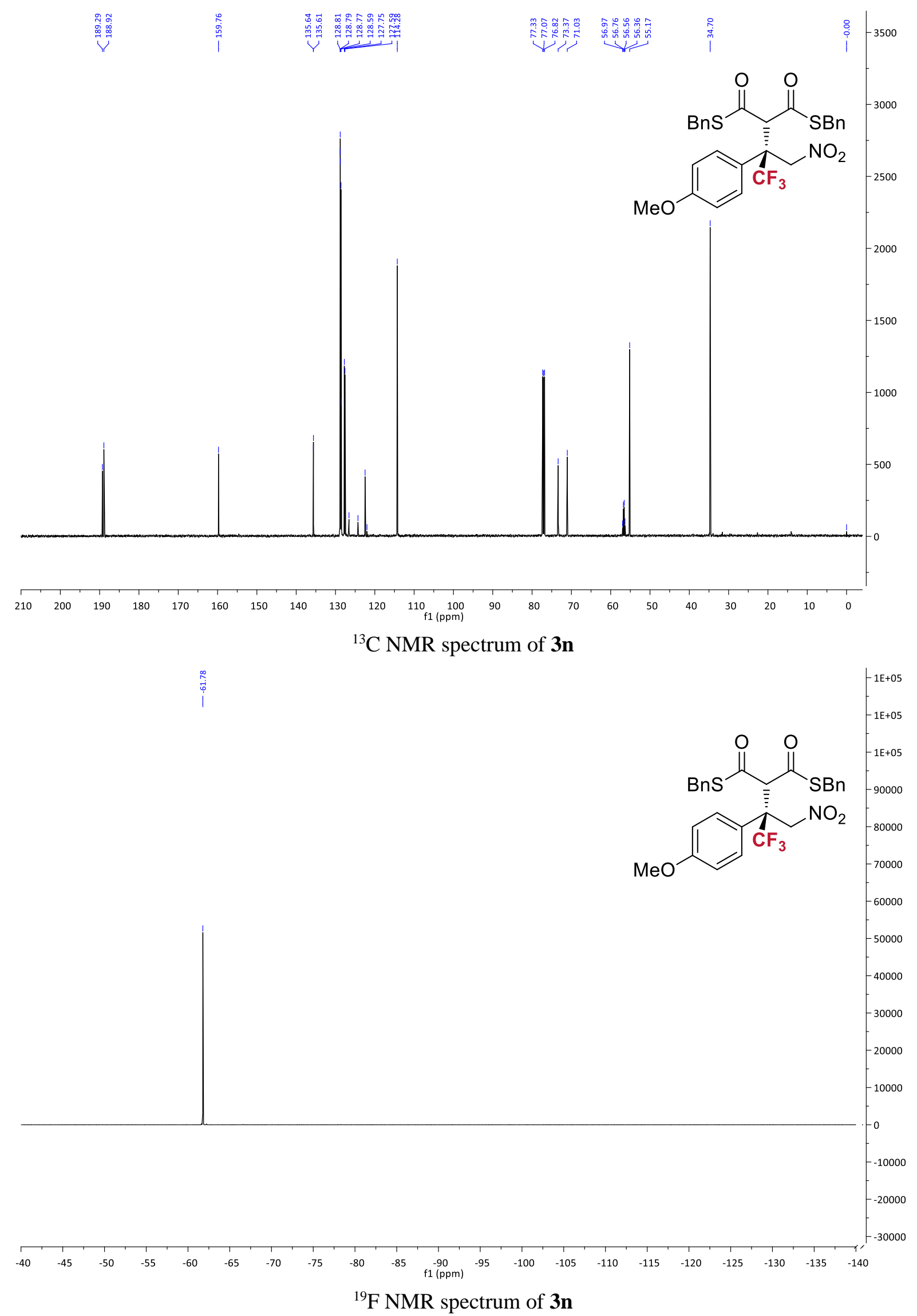




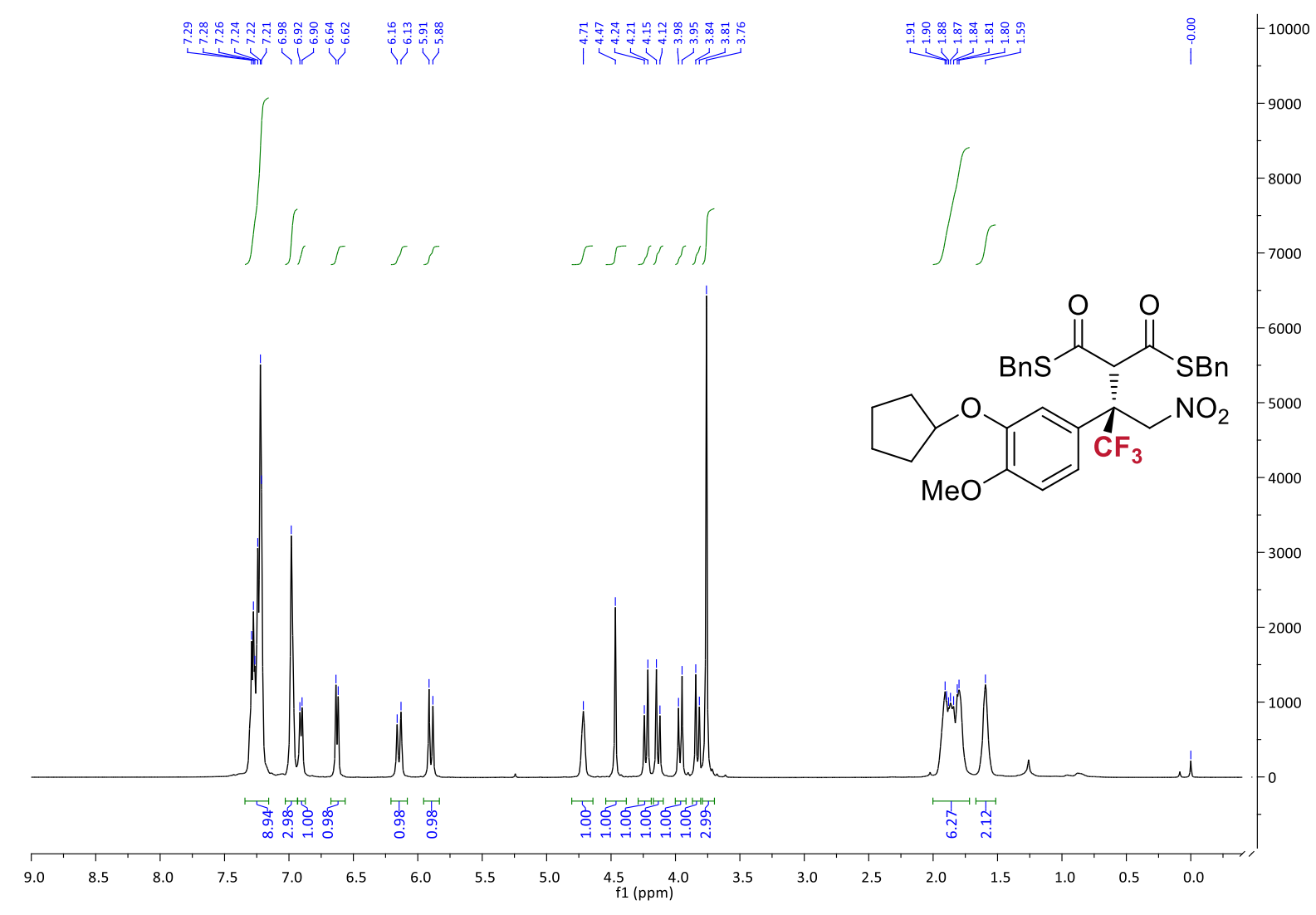

${ }^{1} \mathrm{H}$ NMR spectrum of $\mathbf{3 o}$

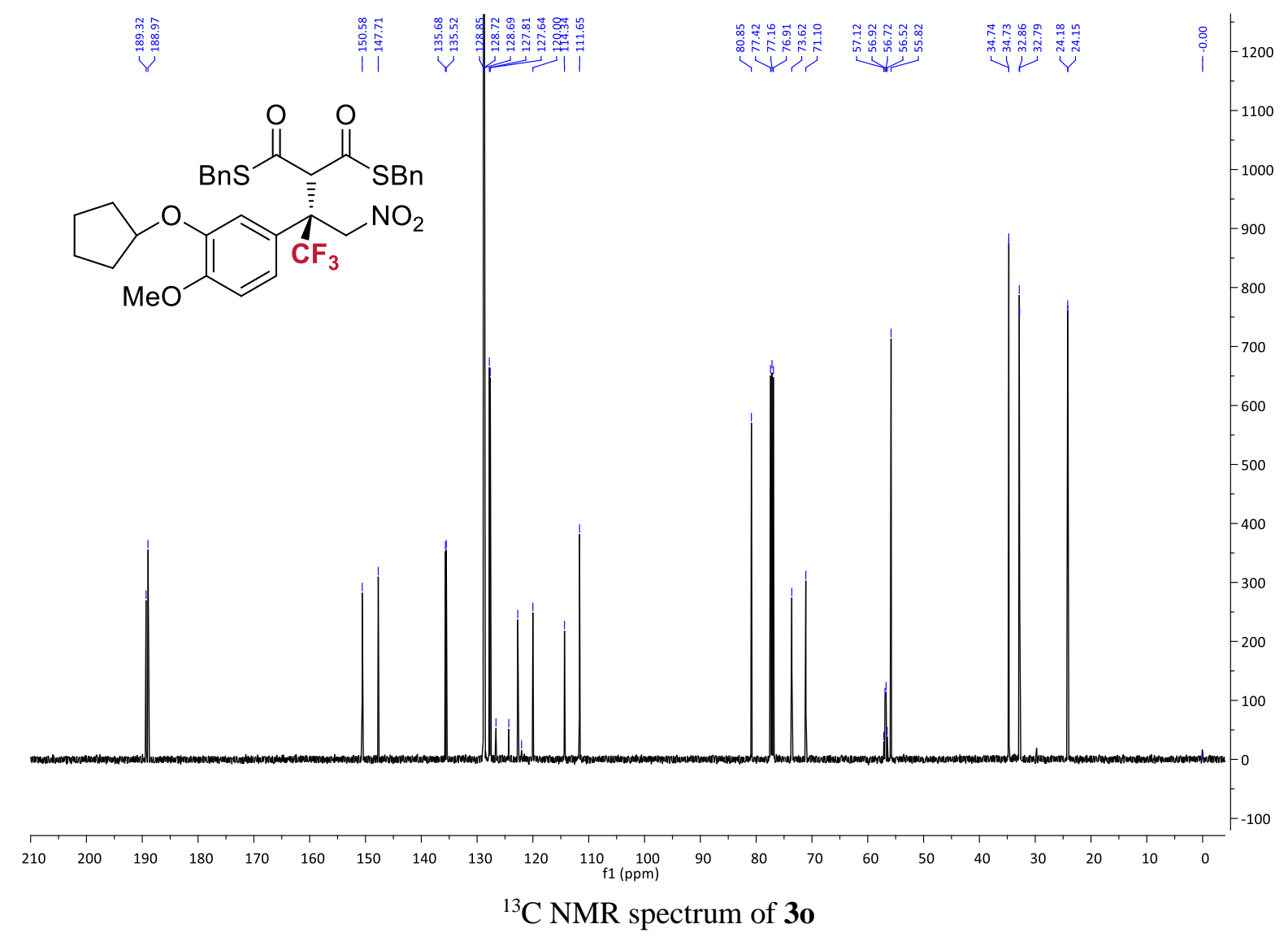




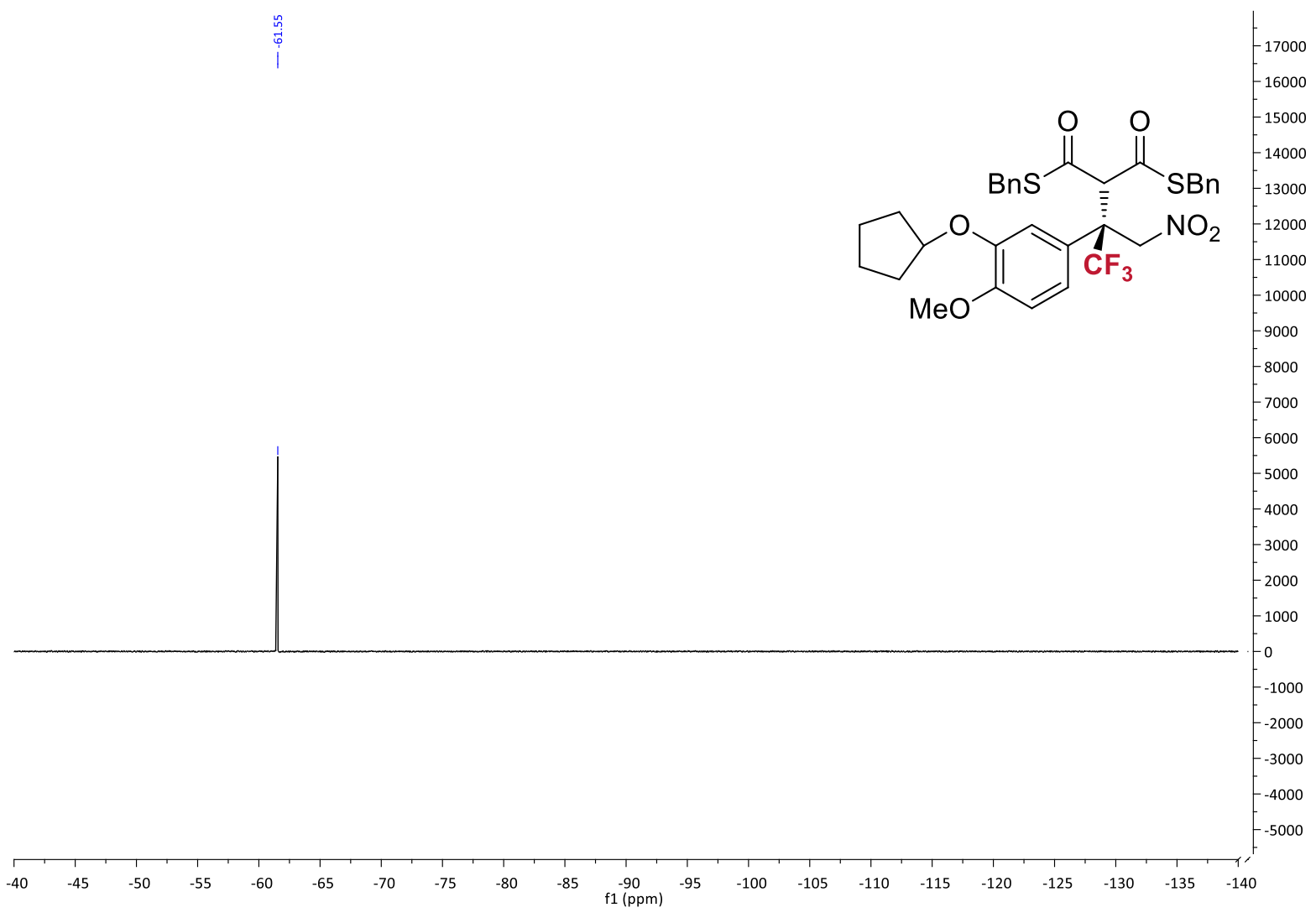

${ }^{19} \mathrm{~F}$ NMR spectrum of $\mathbf{3 o}$

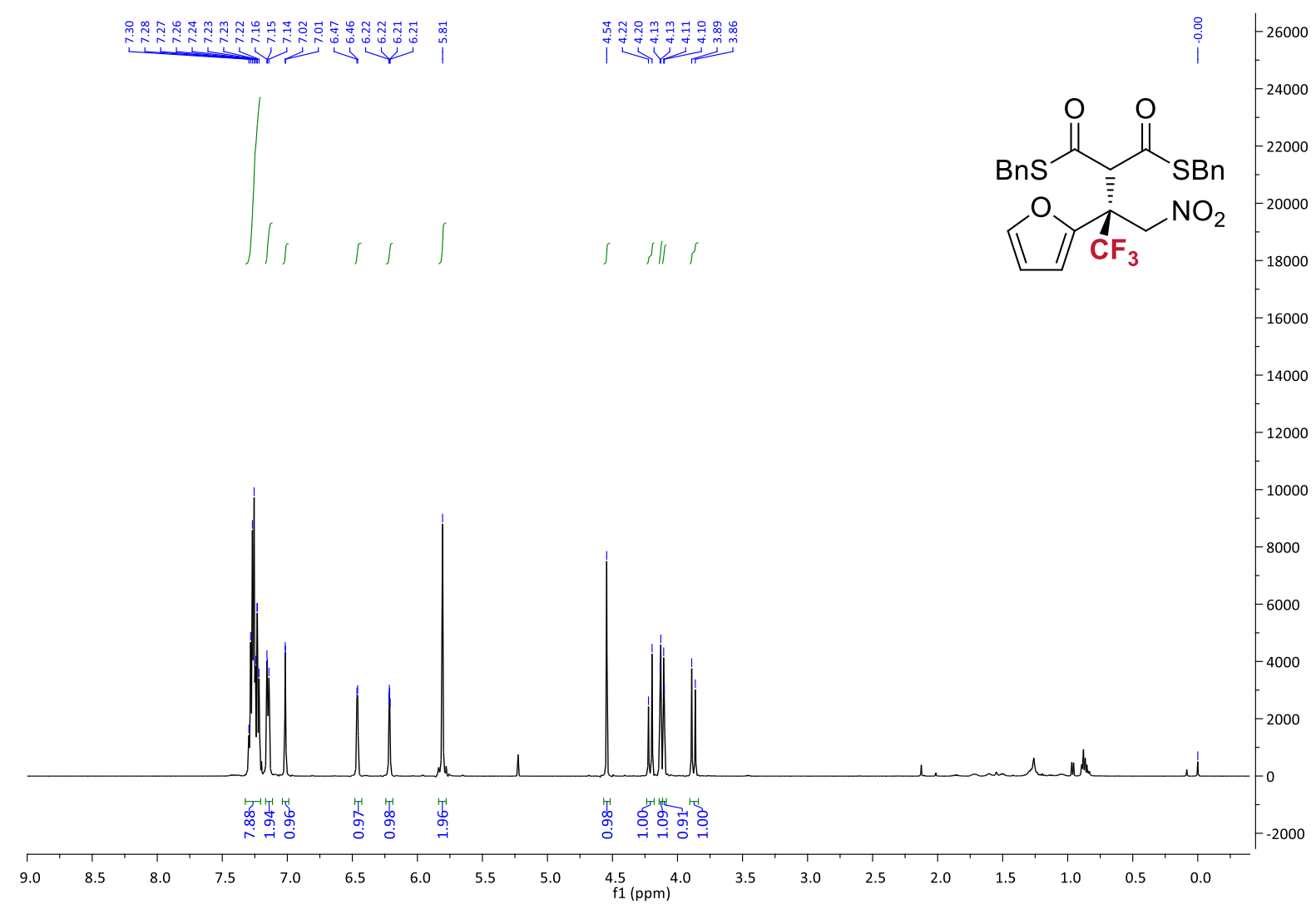

${ }^{1} \mathrm{H}$ NMR spectrum of $\mathbf{3 p}$ 


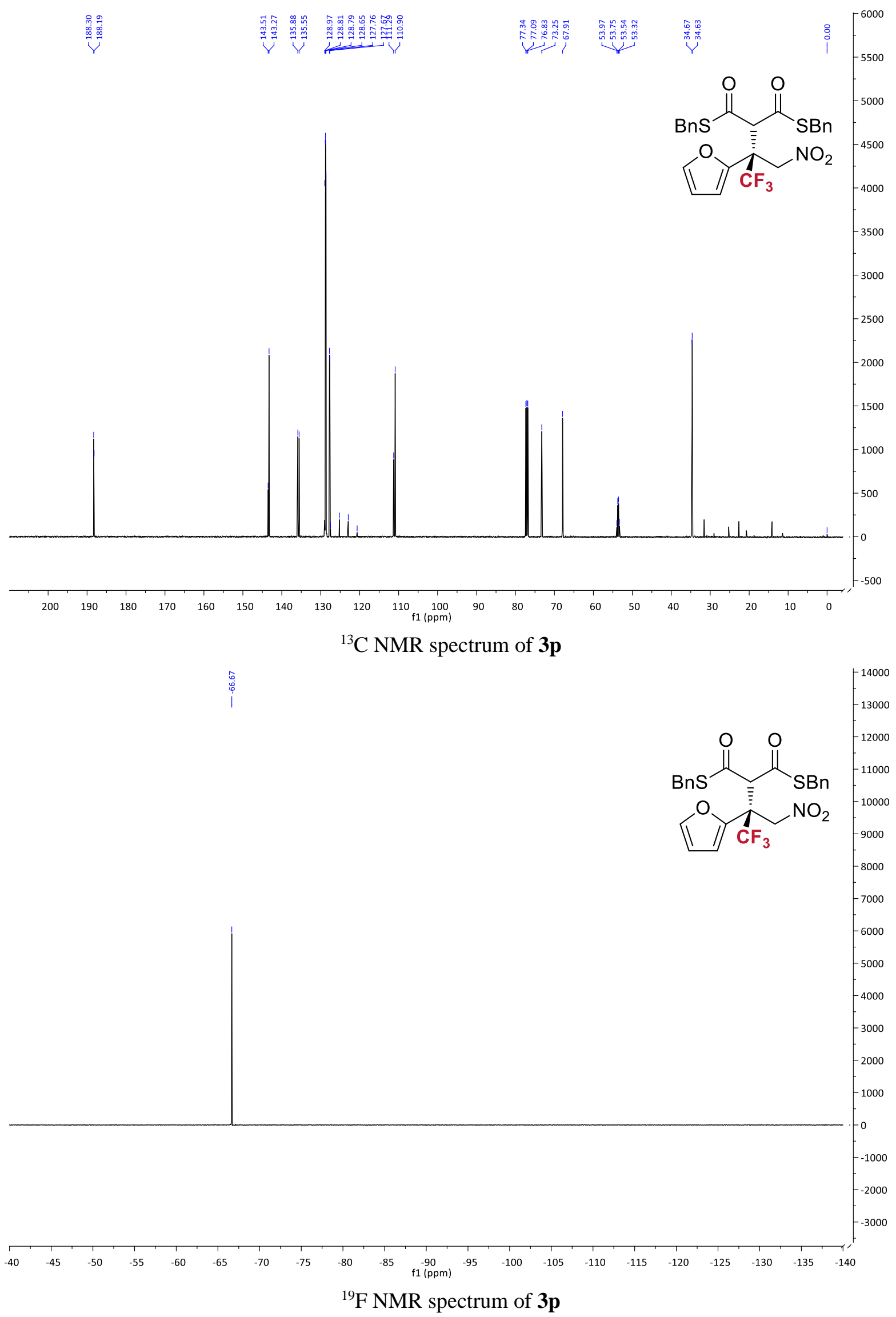




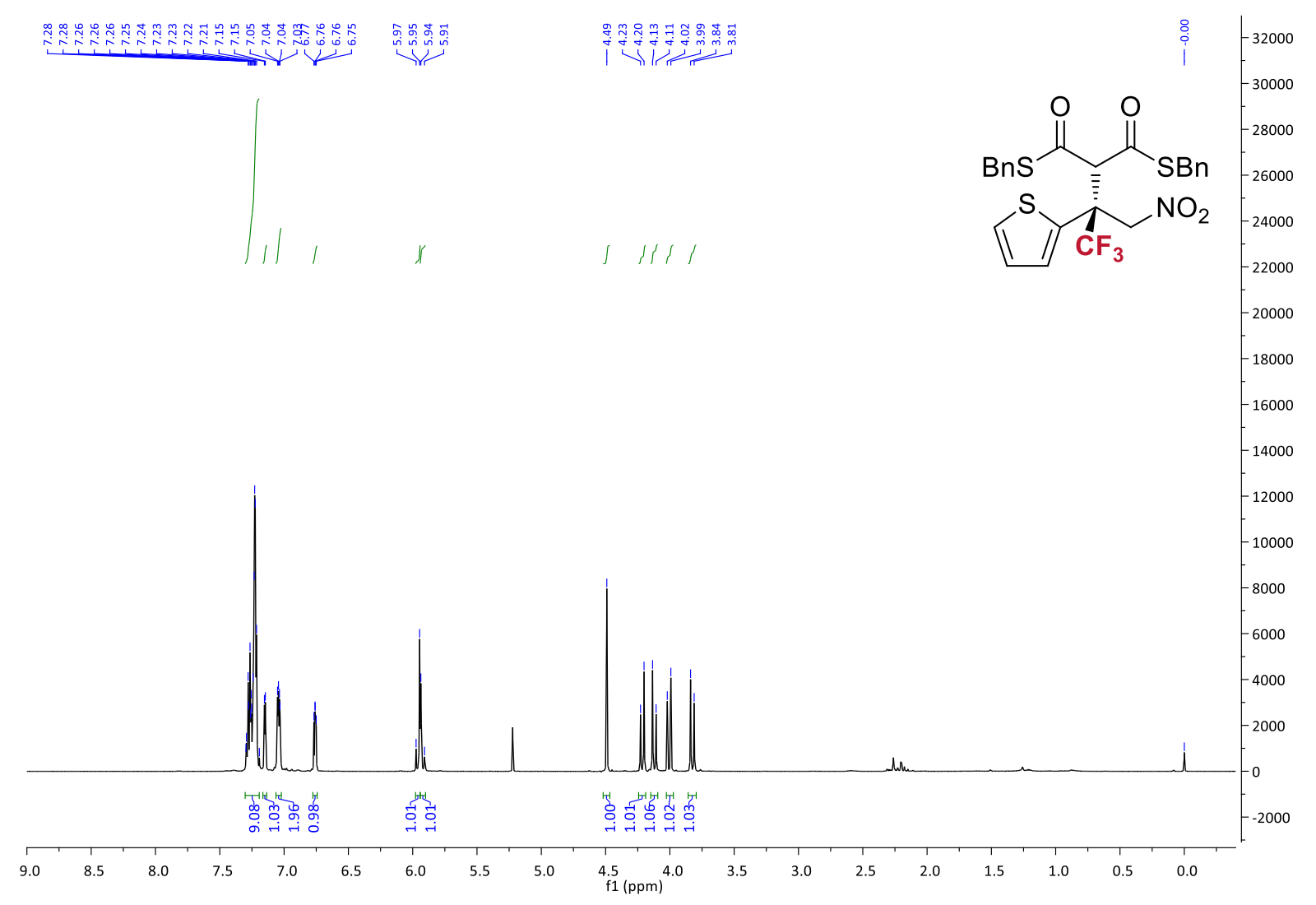

${ }^{1} \mathrm{H}$ NMR spectrum of $\mathbf{3 q}$

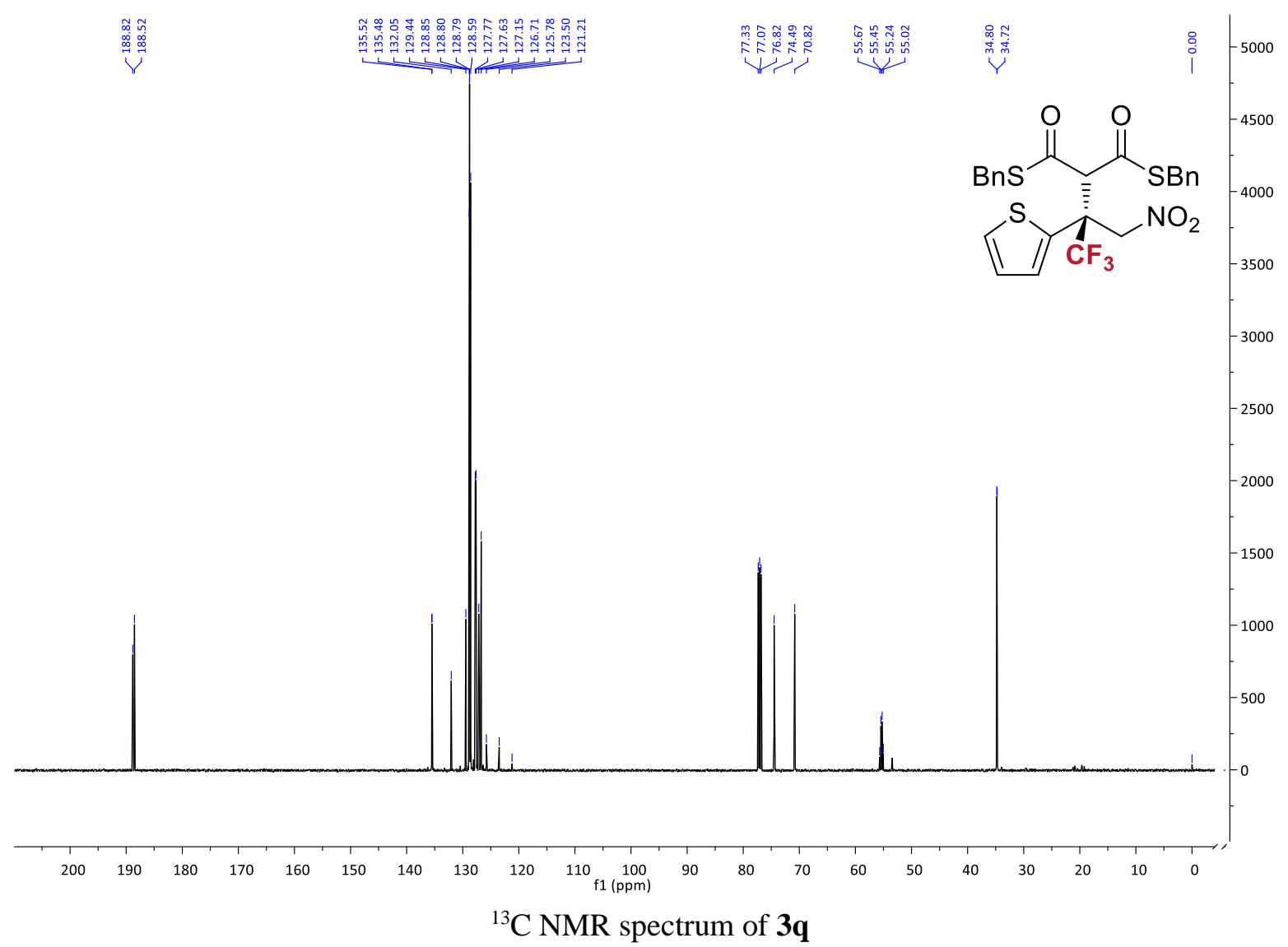




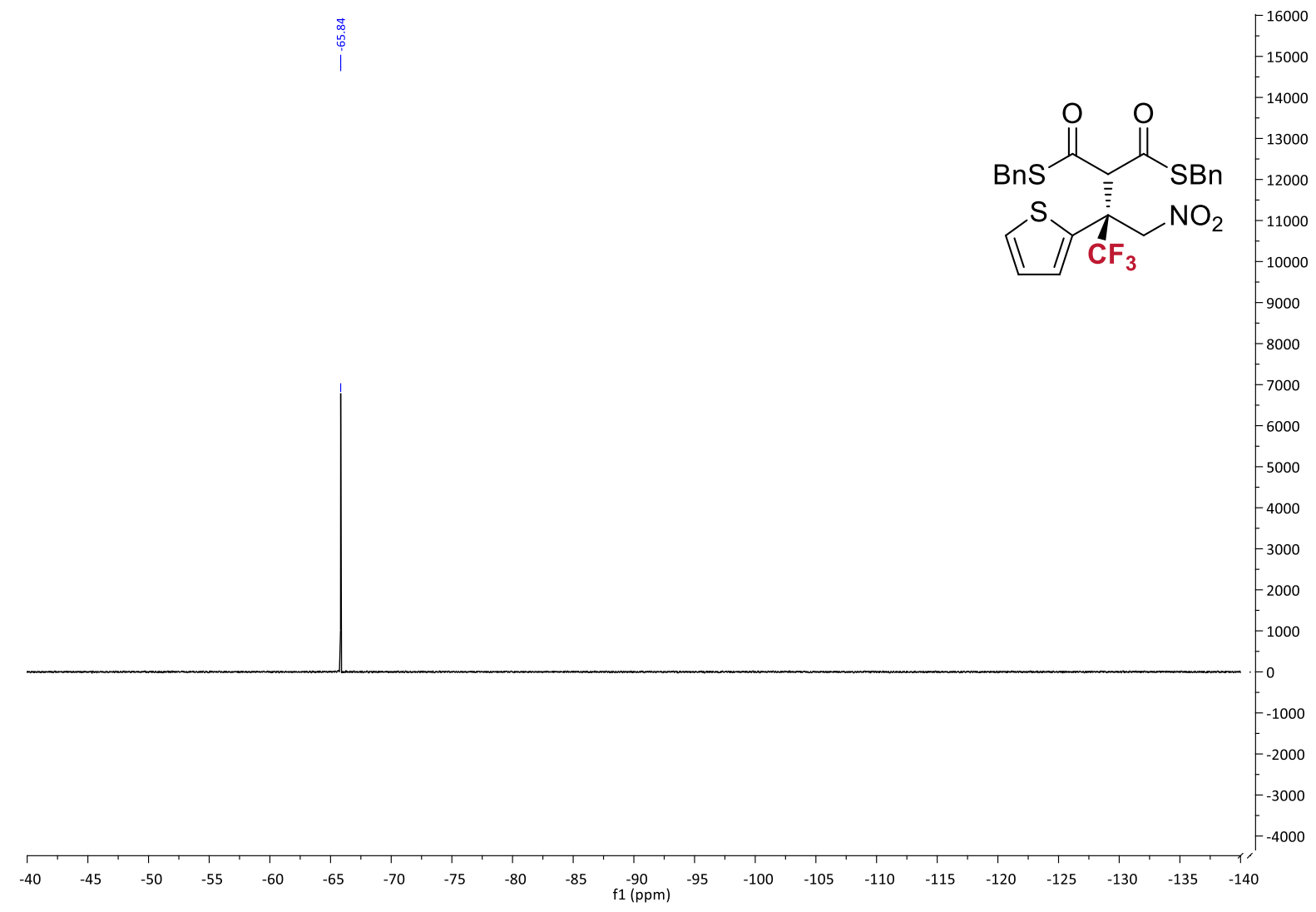

${ }^{19} \mathrm{~F}$ NMR spectrum of $\mathbf{3 q}$

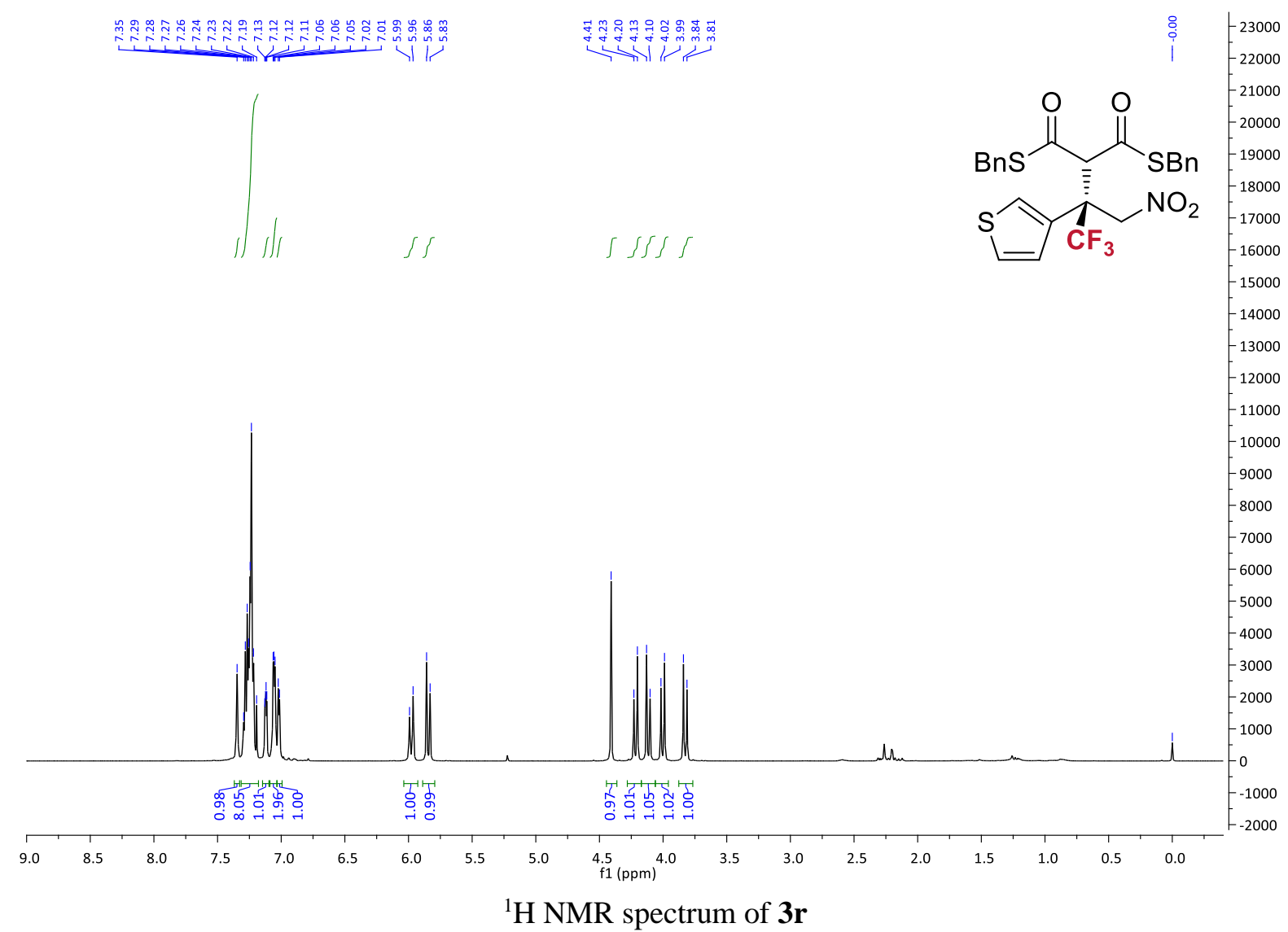




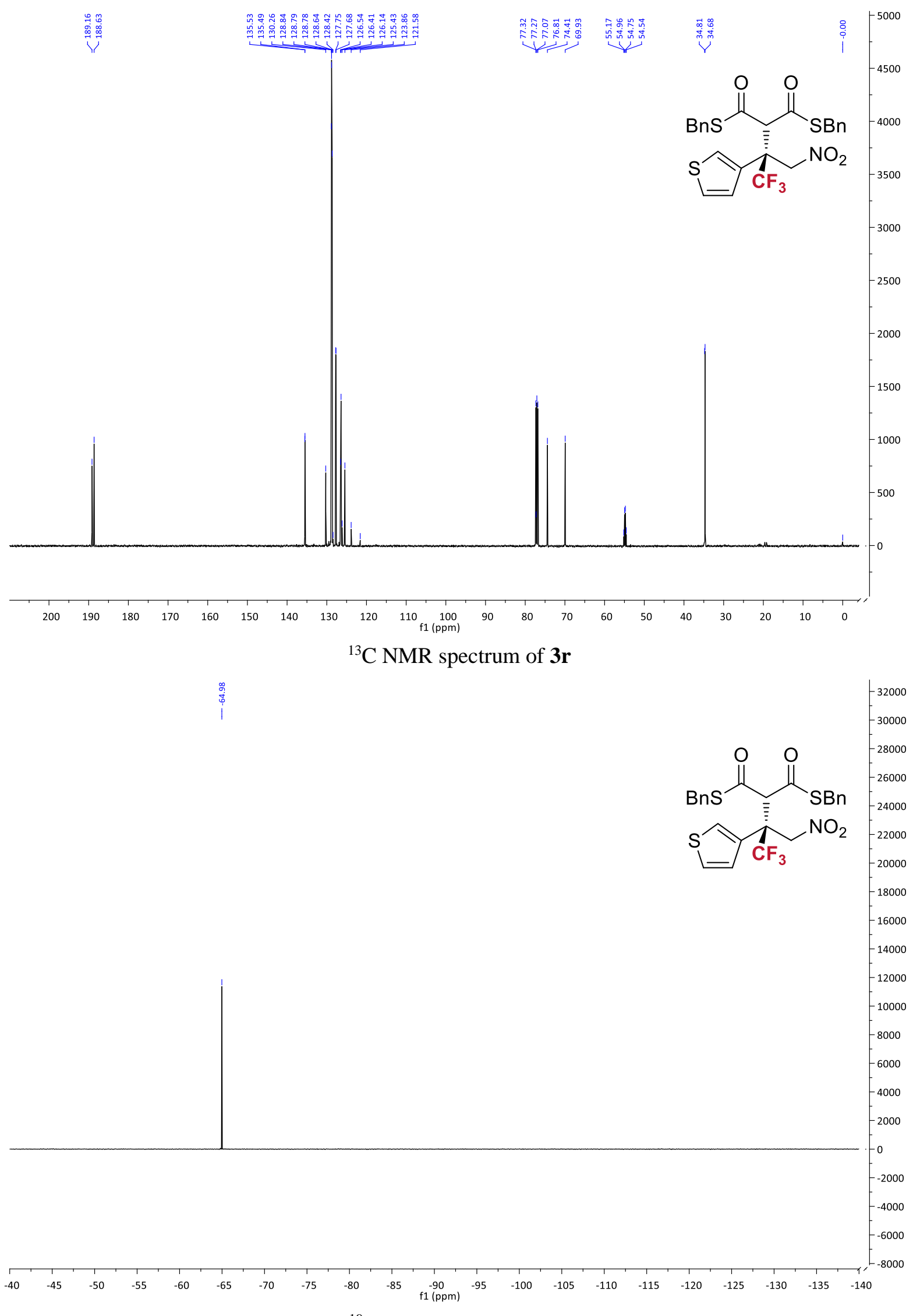

${ }^{19} \mathrm{~F}$ NMR spectrum of $\mathbf{3 r}$ 


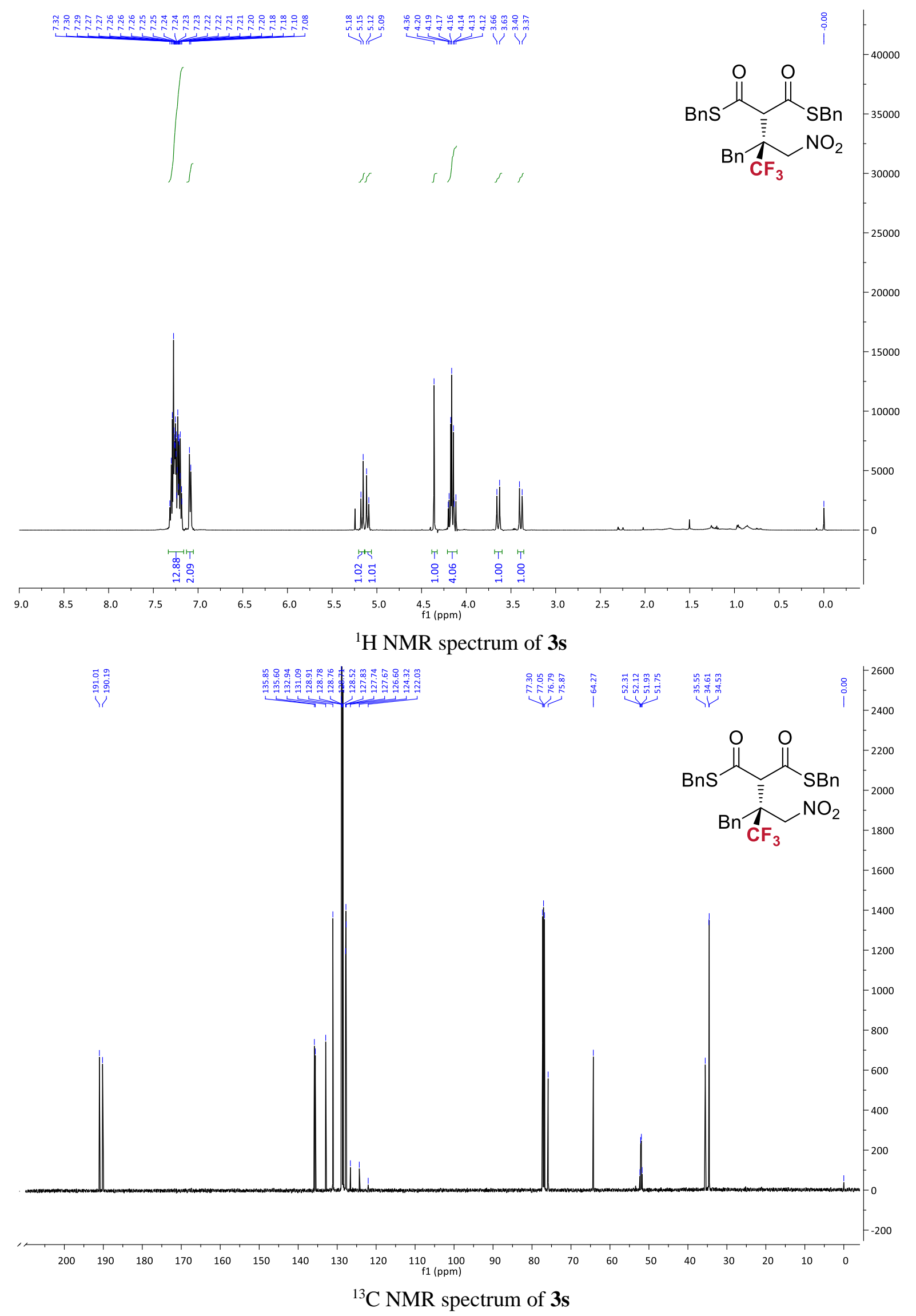




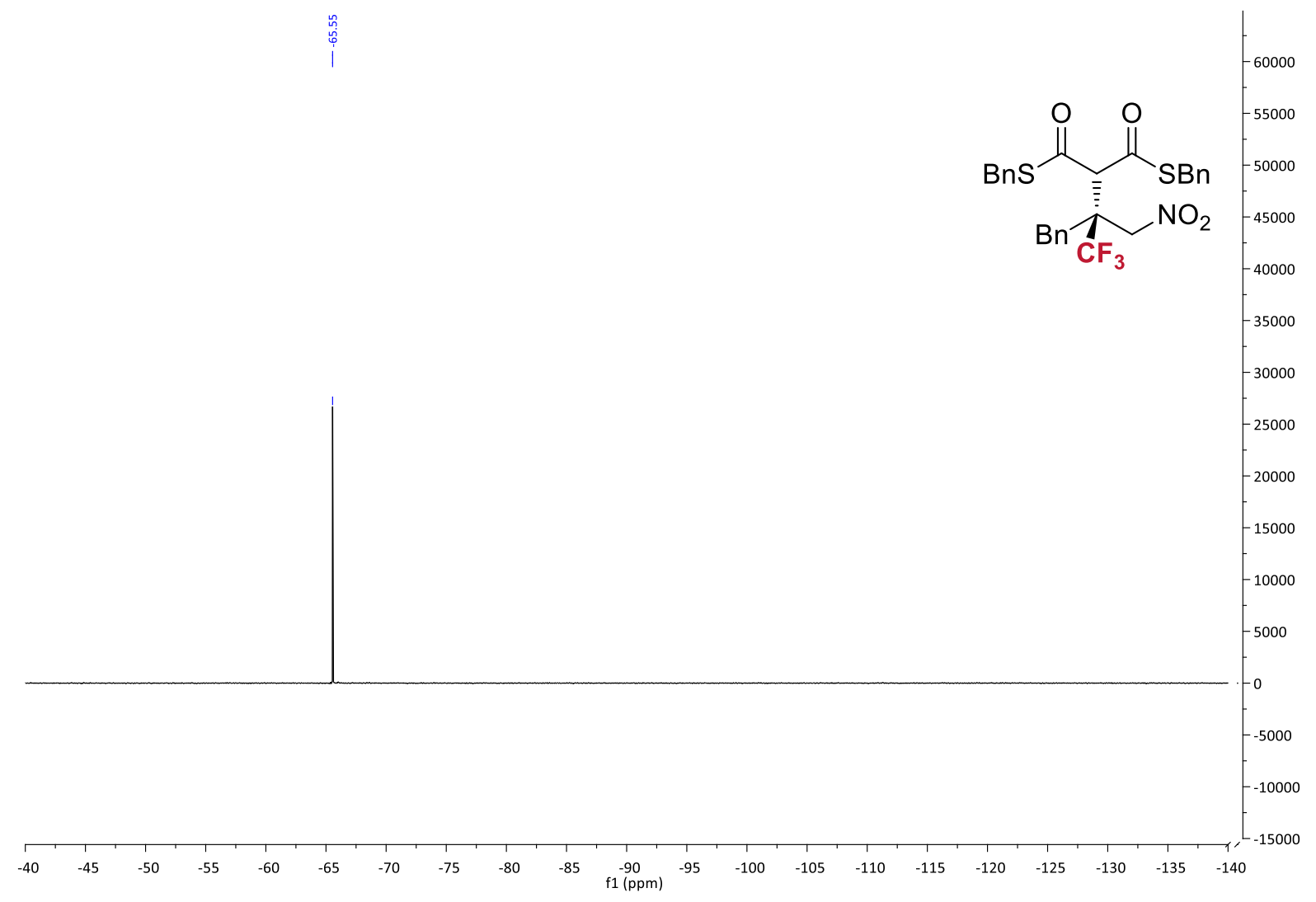

${ }^{19} \mathrm{~F}$ NMR spectrum of $\mathbf{3 s}$

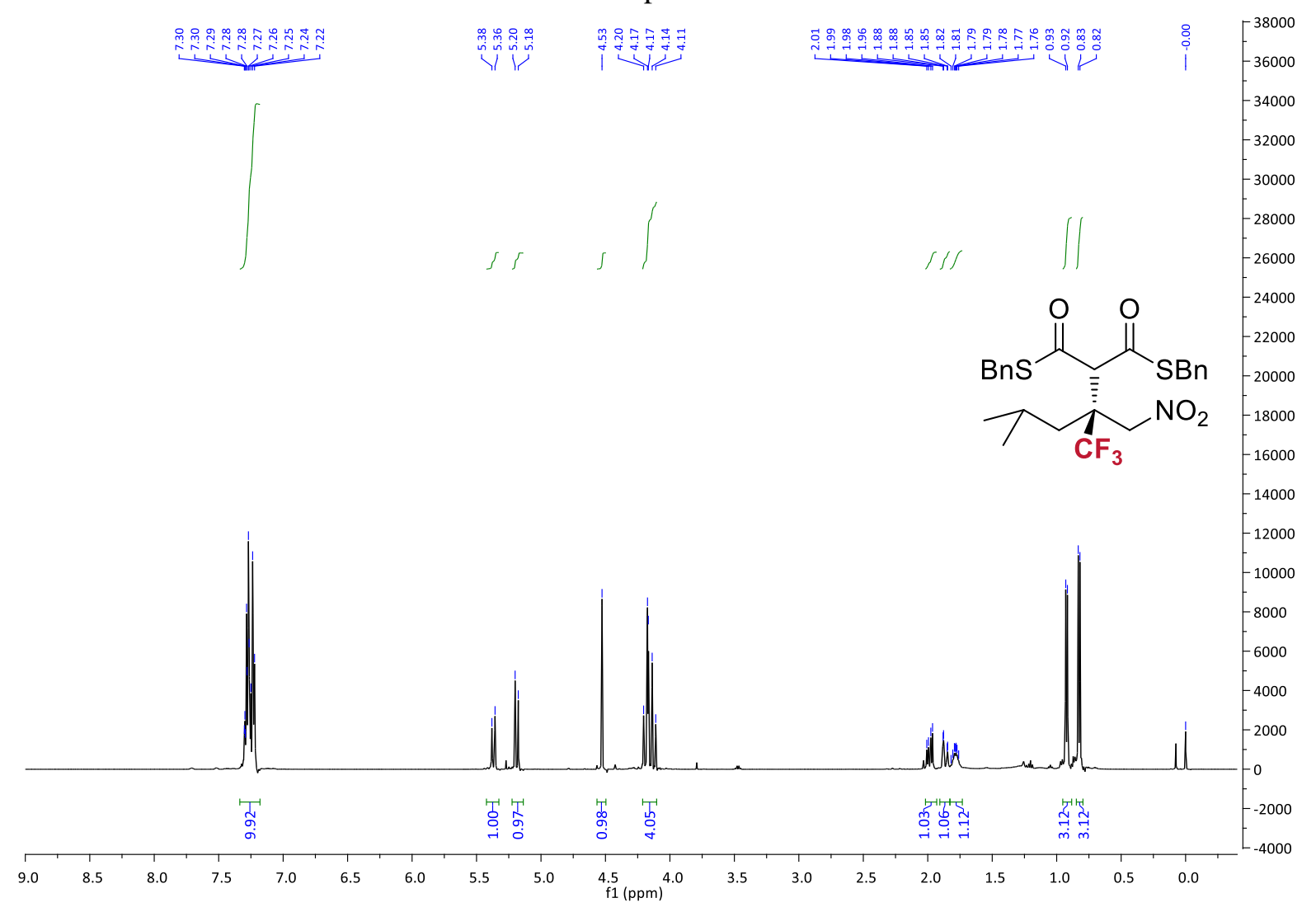

${ }^{1} \mathrm{H}$ NMR spectrum of $\mathbf{3 t}$ 


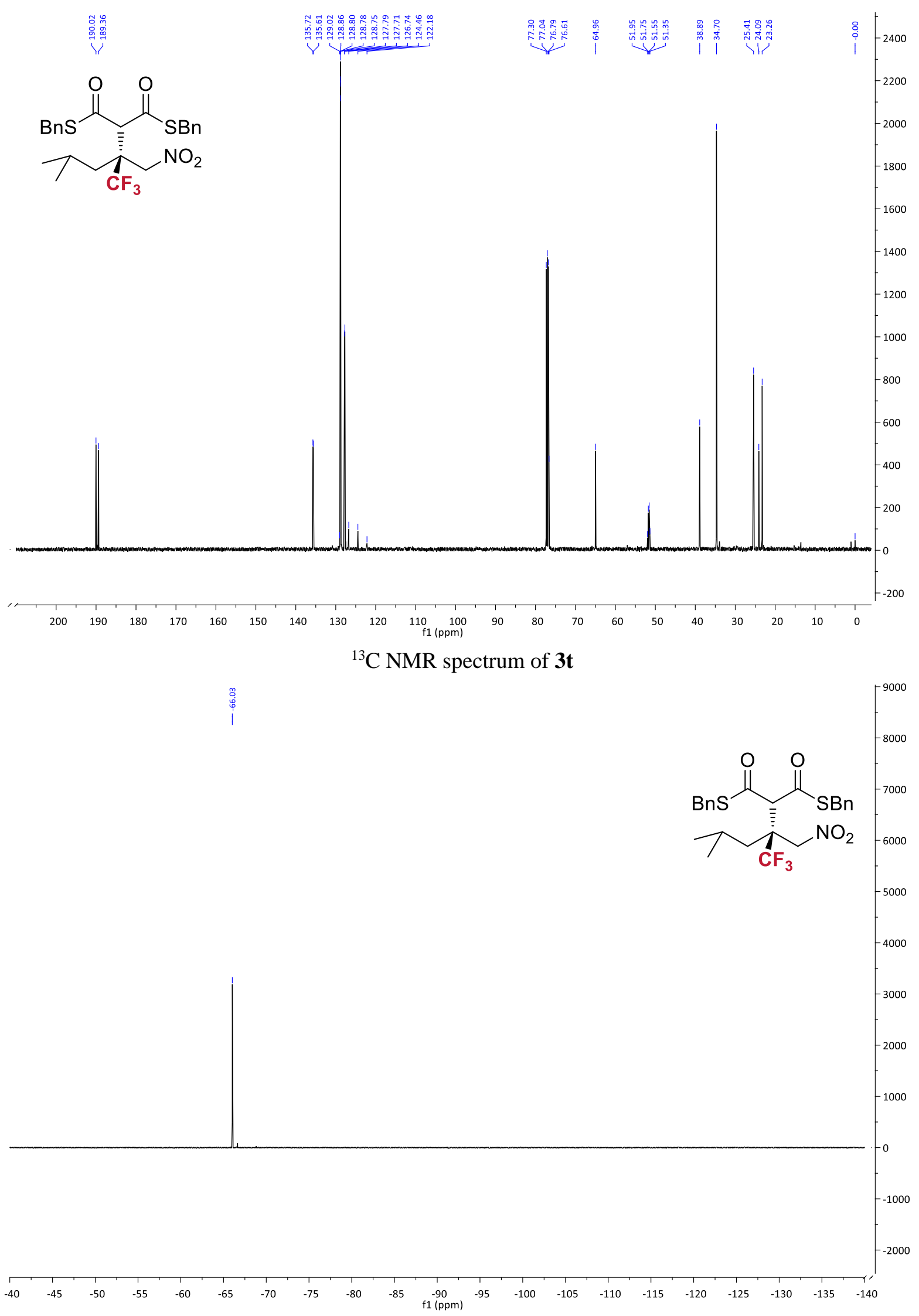

${ }^{19} \mathrm{~F}$ NMR spectrum of $\mathbf{3 t}$ 


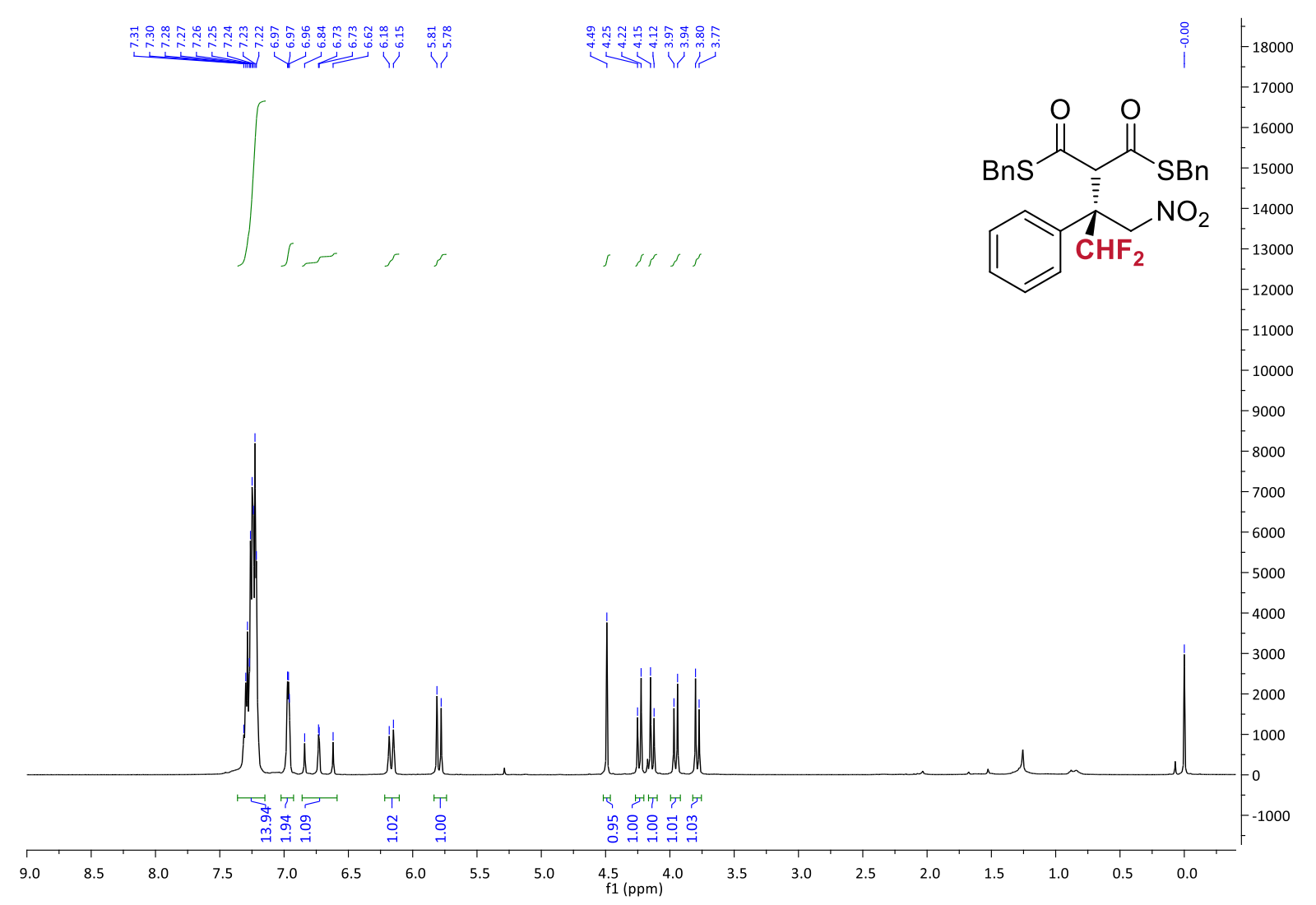

${ }^{1} \mathrm{H}$ NMR spectrum of $\mathbf{3 u}$

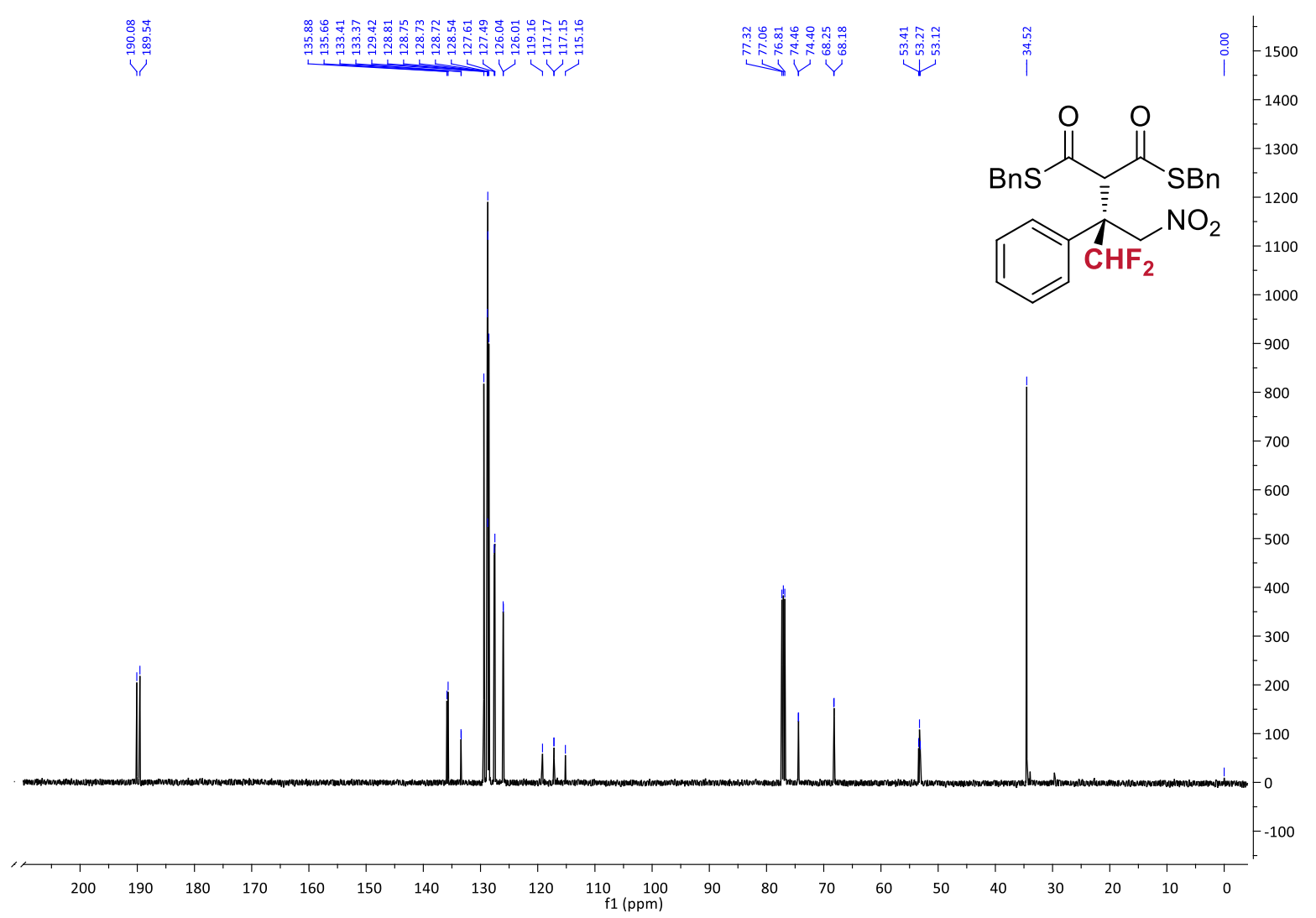

${ }^{13} \mathrm{C}$ NMR spectrum of $\mathbf{3 u}$ 


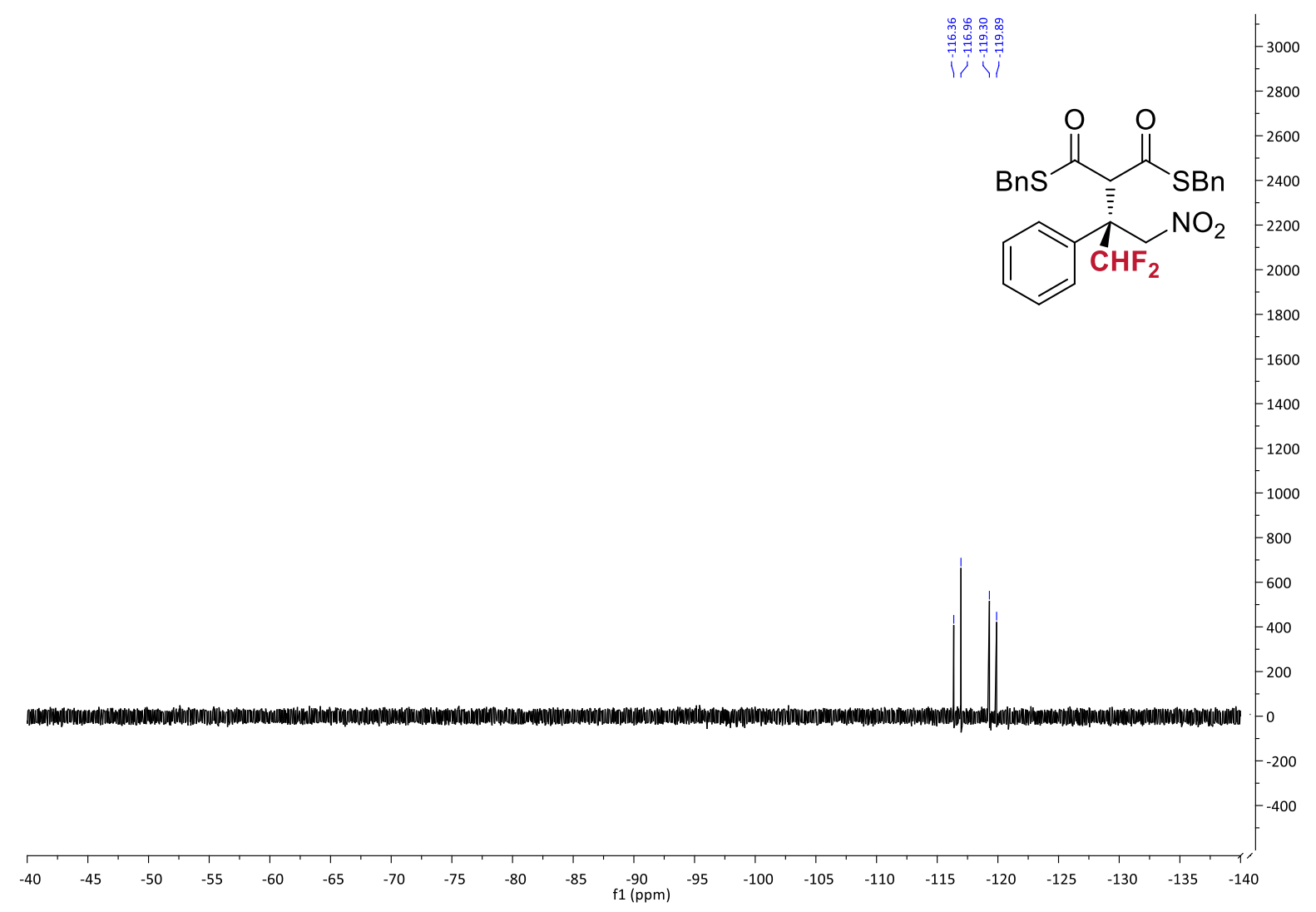

${ }^{19} \mathrm{~F}$ NMR spectrum of $\mathbf{3 u}$

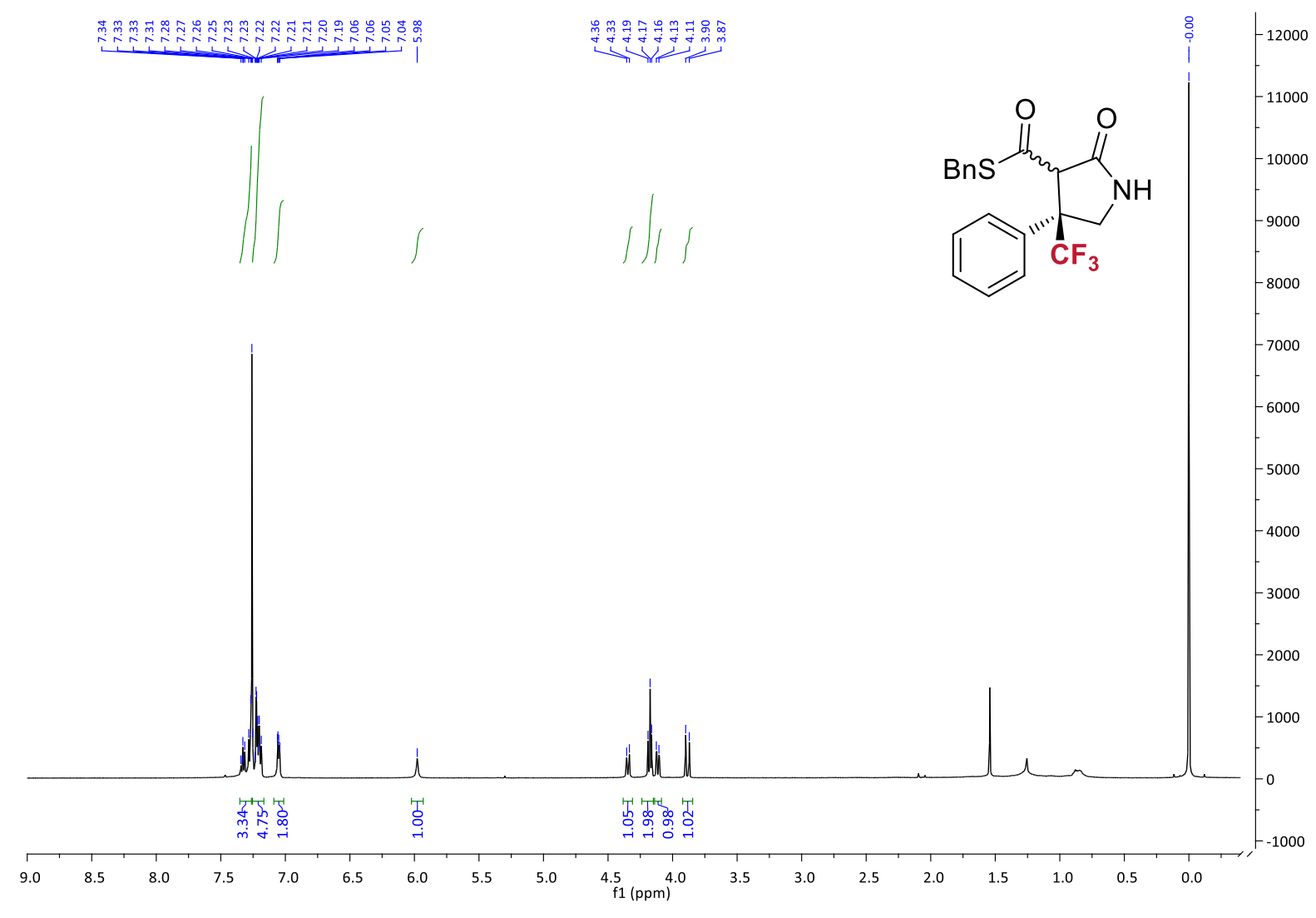

${ }^{1} \mathrm{H}$ NMR spectrum of $\mathbf{5 a}$ 


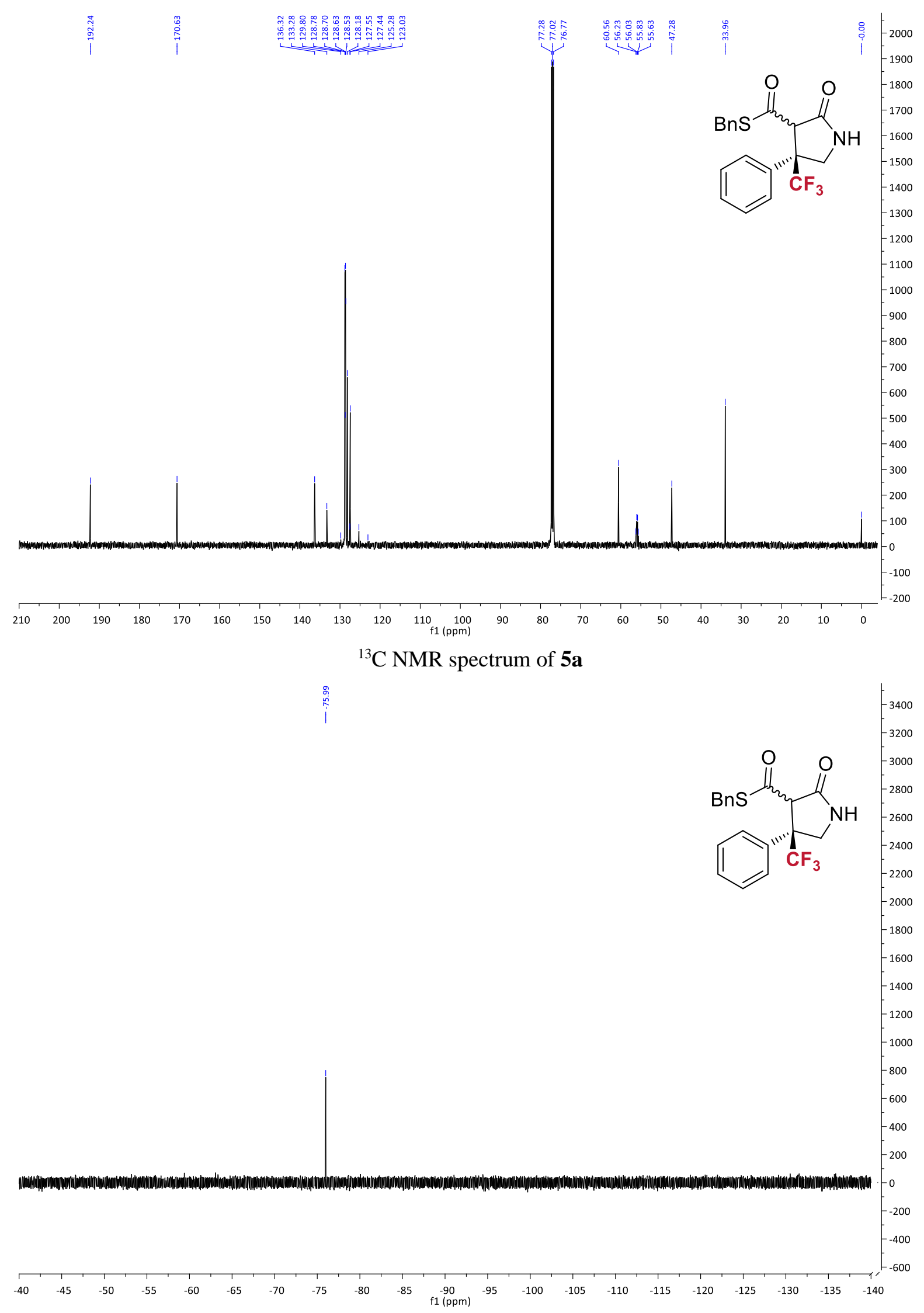

${ }^{19} \mathrm{~F}$ NMR spectrum of $\mathbf{5 a}$ 


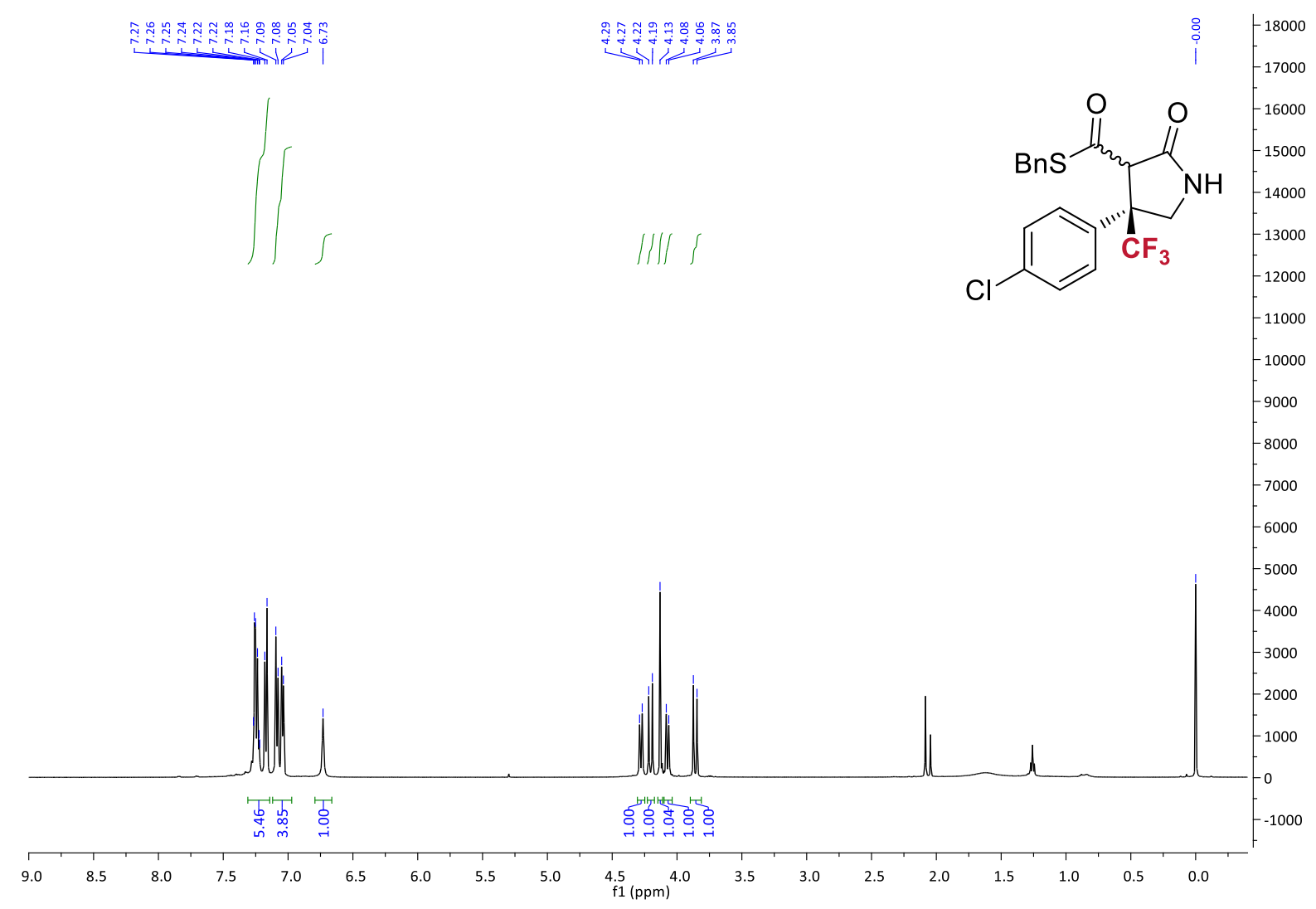

${ }^{1} \mathrm{H}$ NMR spectrum of $\mathbf{5 f}$

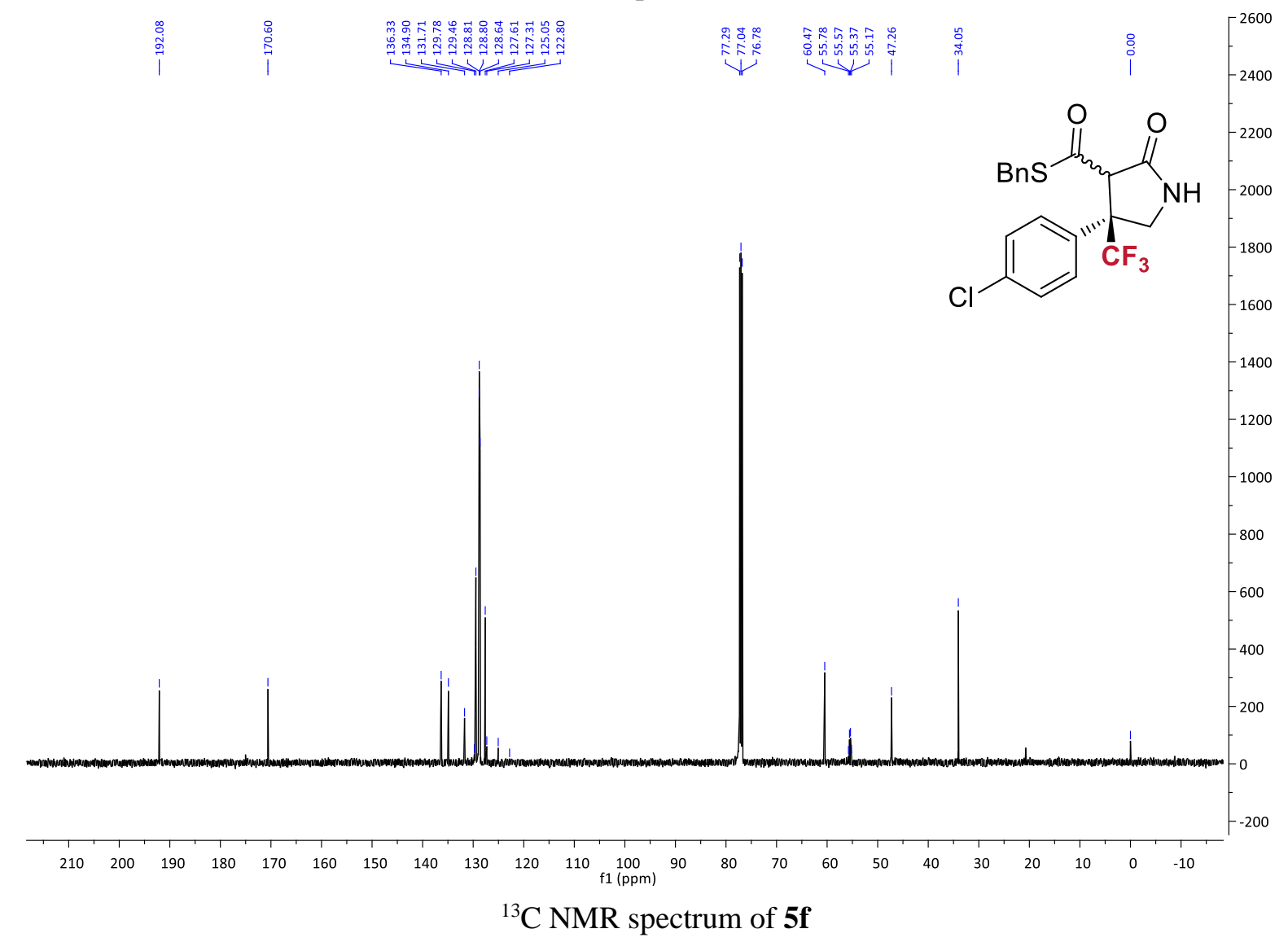




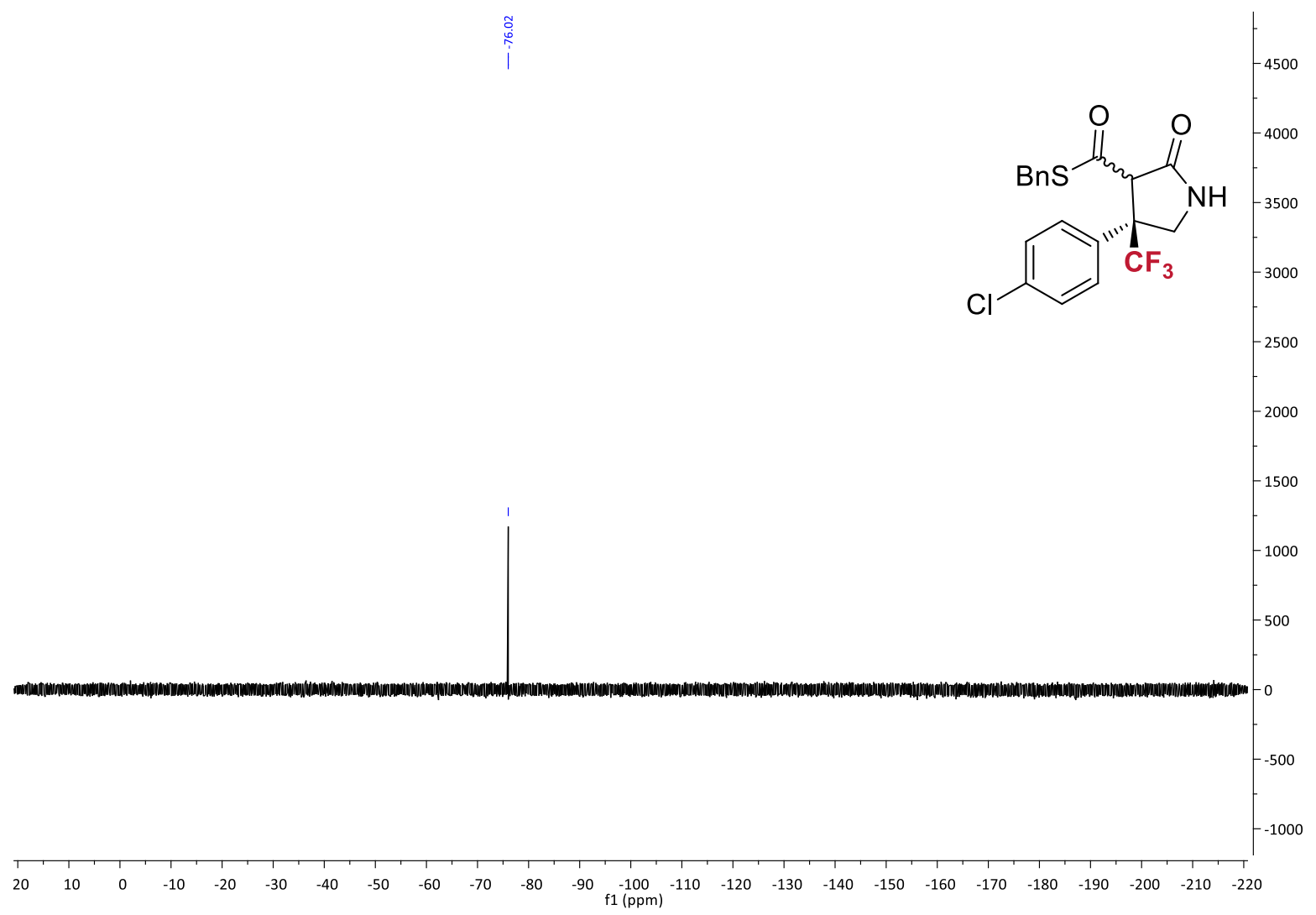

${ }^{19} \mathrm{~F}$ NMR spectrum of $\mathbf{5 f}$

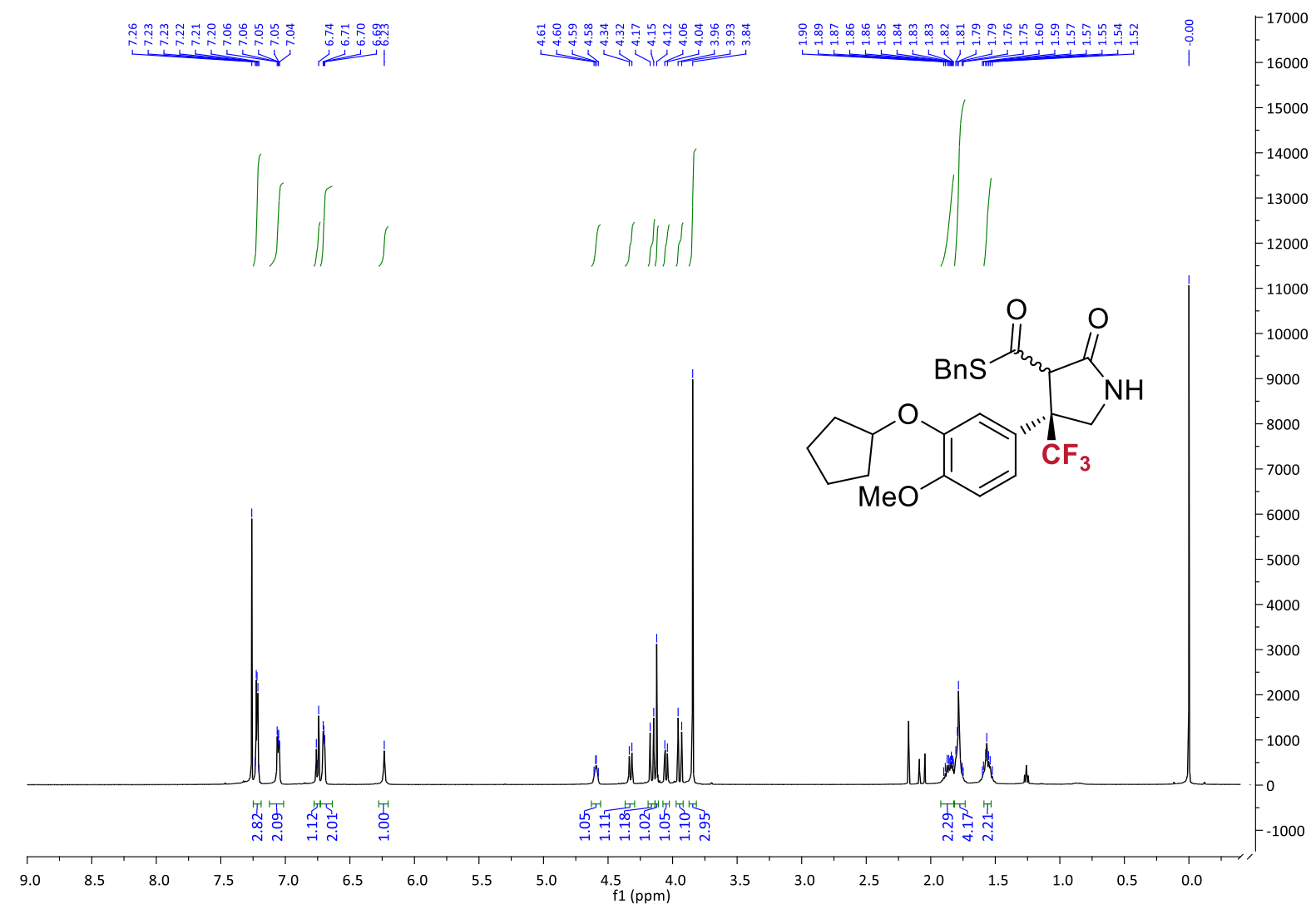

${ }^{1} \mathrm{H}$ NMR spectrum of $\mathbf{5 0}$ 


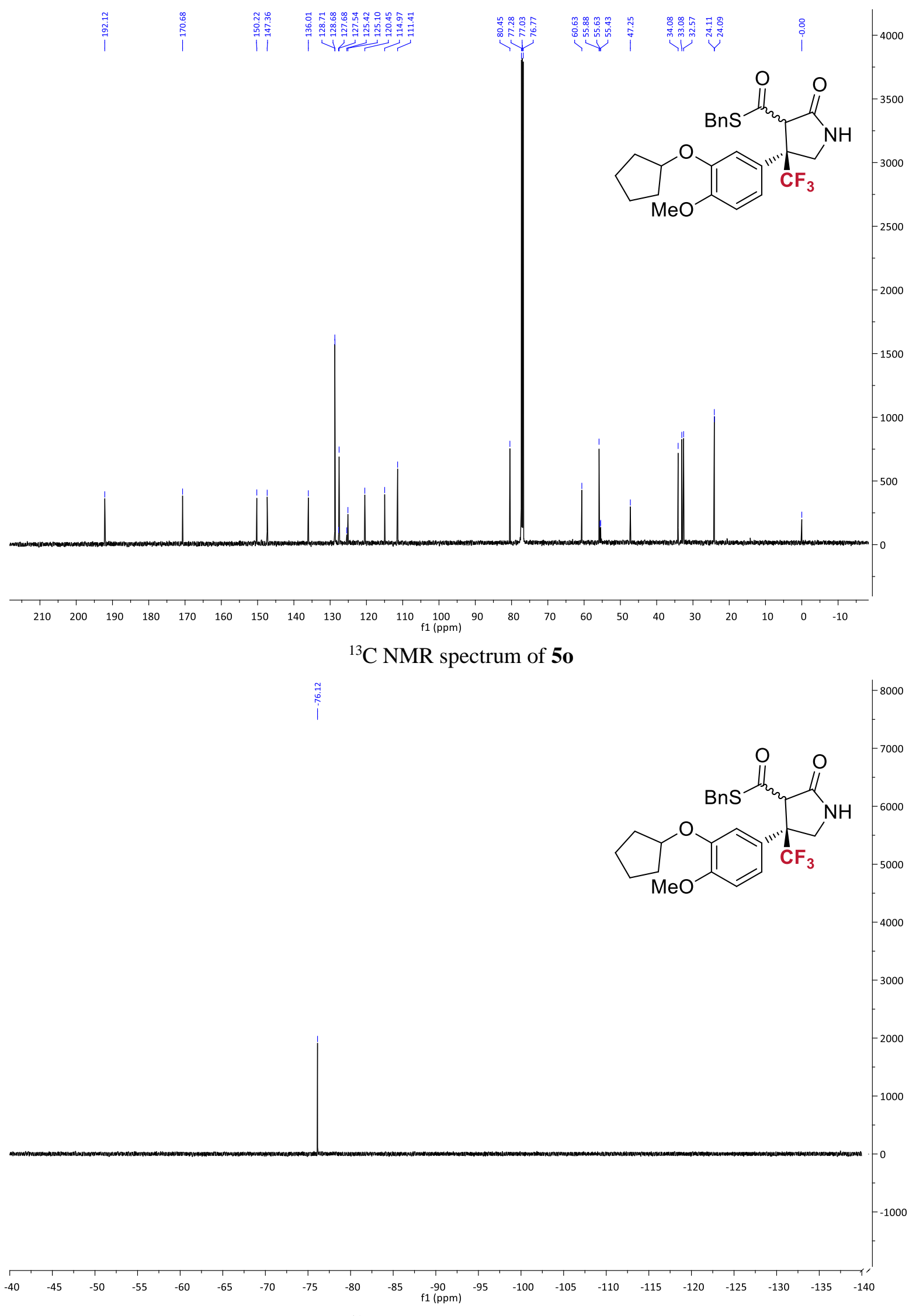

${ }^{19} \mathrm{~F}$ NMR spectrum of $\mathbf{5 0}$ 


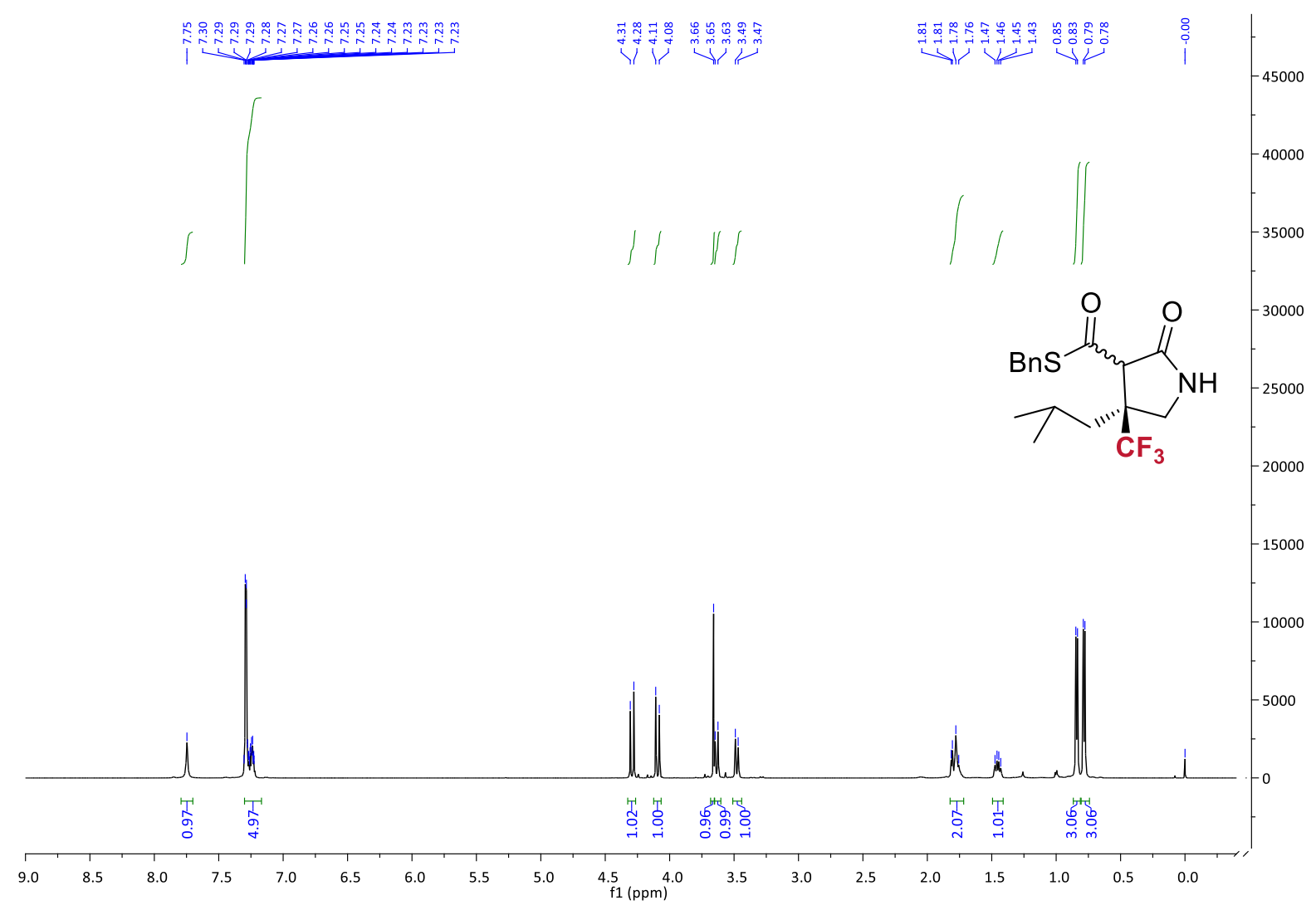

${ }^{1} \mathrm{H}$ NMR spectrum of $\mathbf{5 t}$

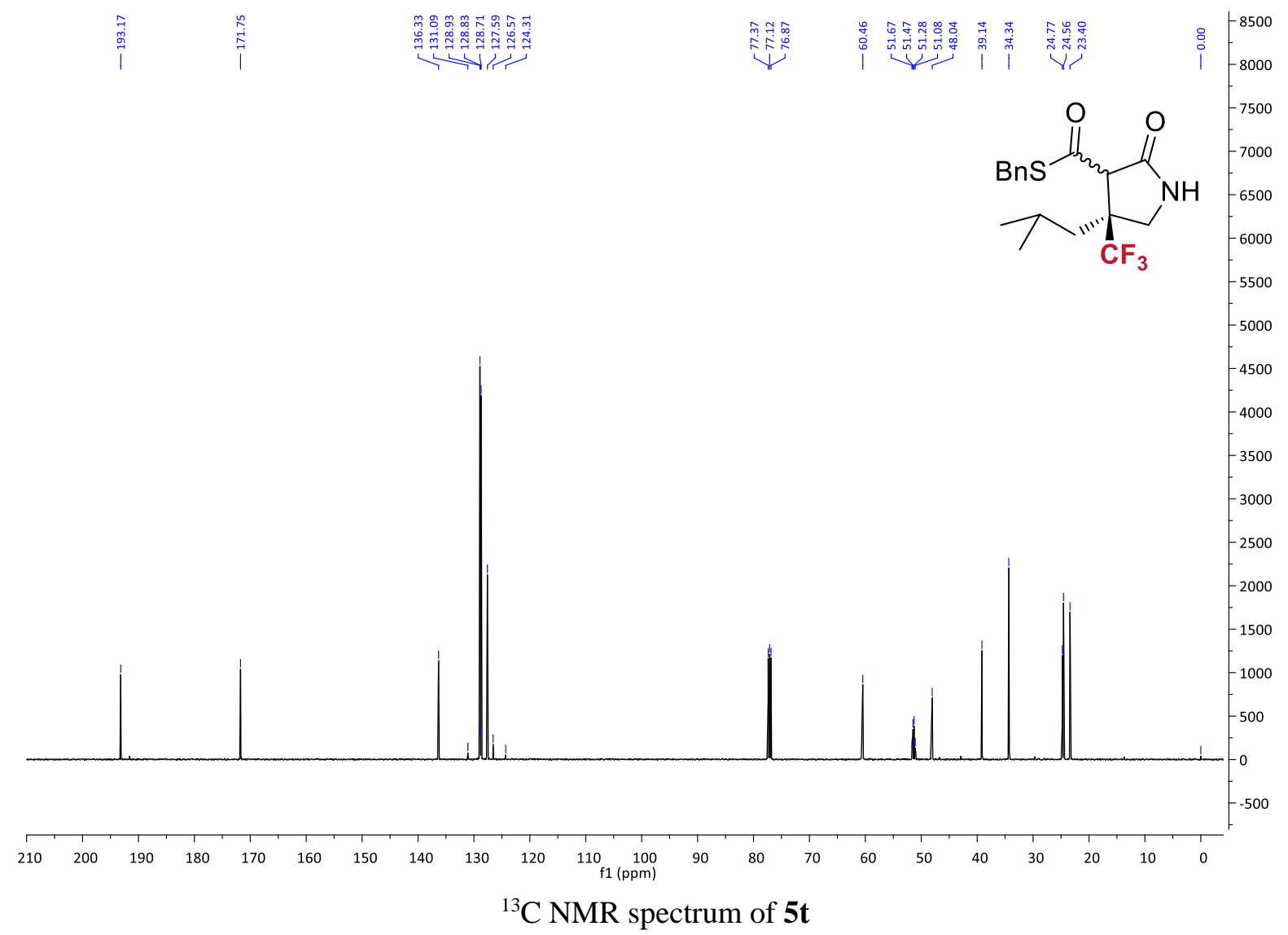




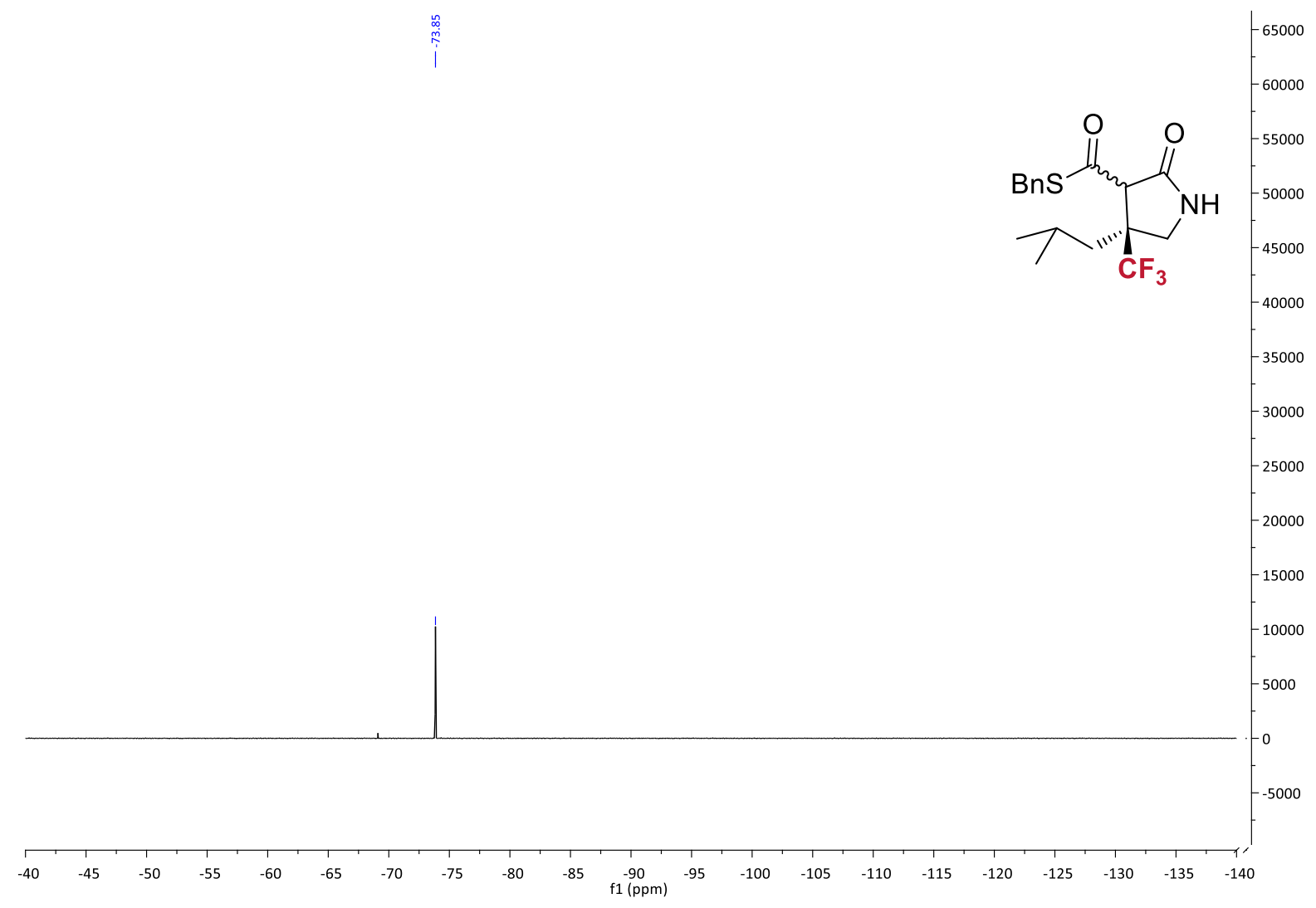

${ }^{19} \mathrm{~F}$ NMR spectrum of $\mathbf{5 t}$

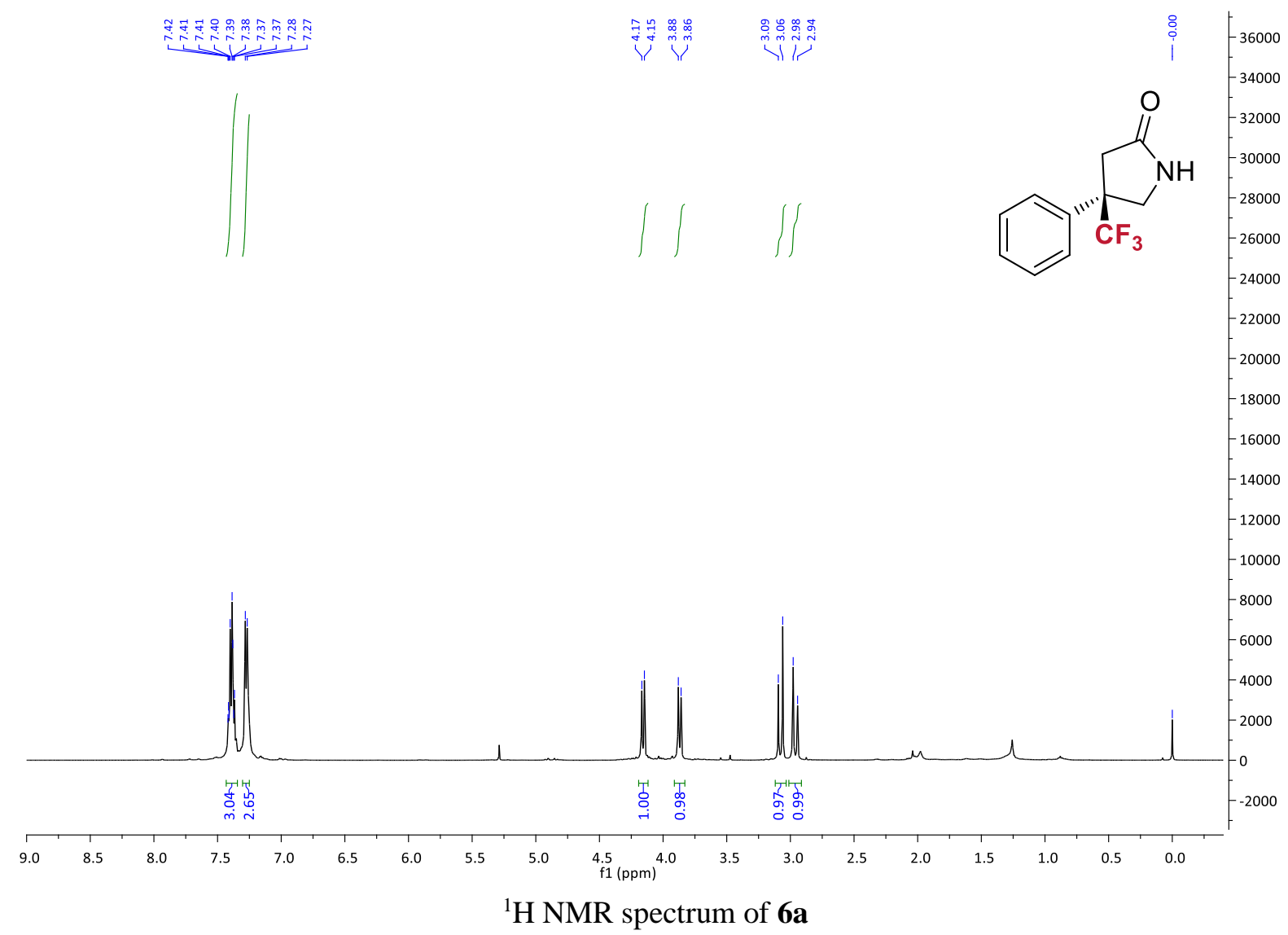




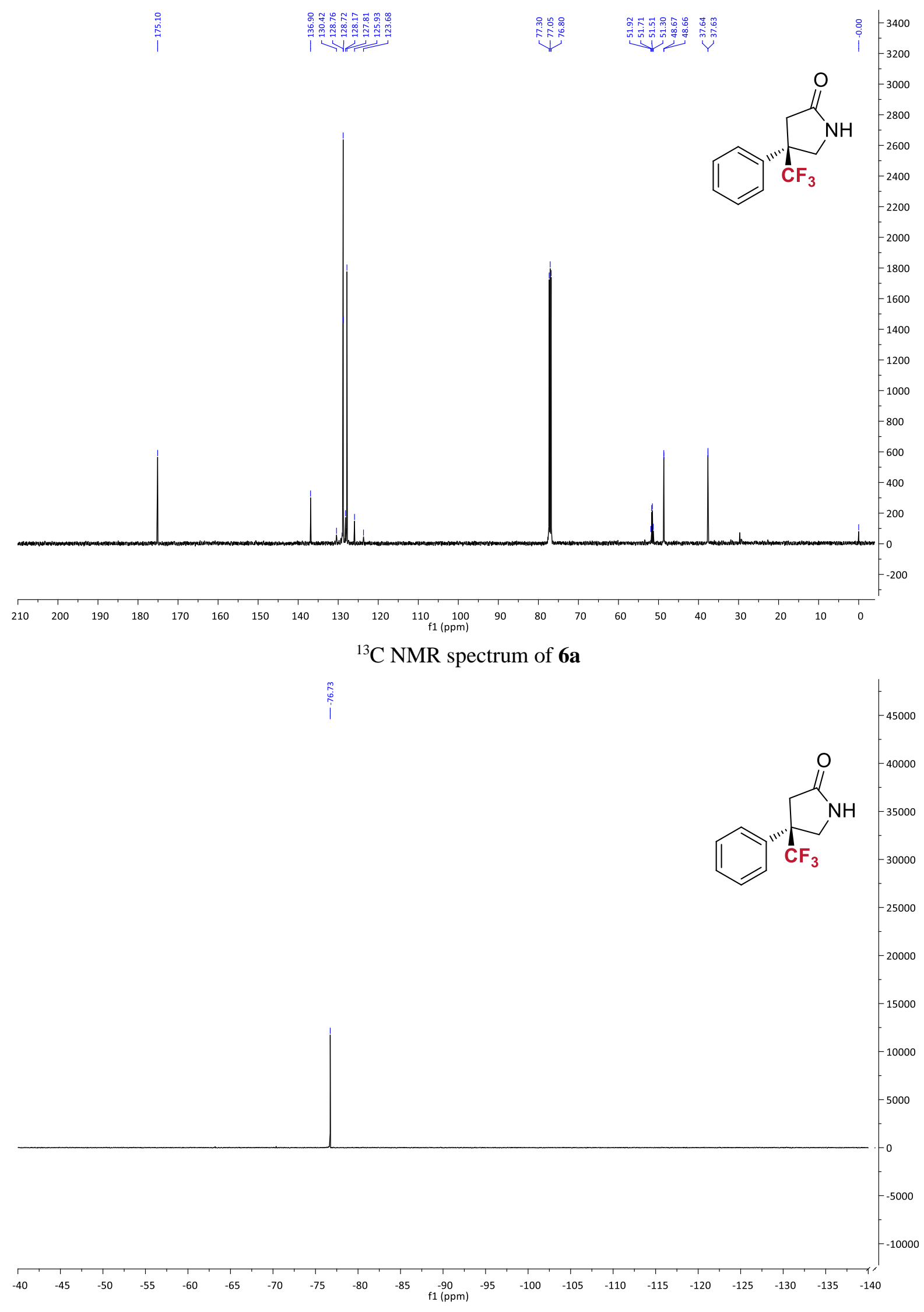

${ }^{19} \mathrm{~F}$ NMR spectrum of $\mathbf{6 a}$ 


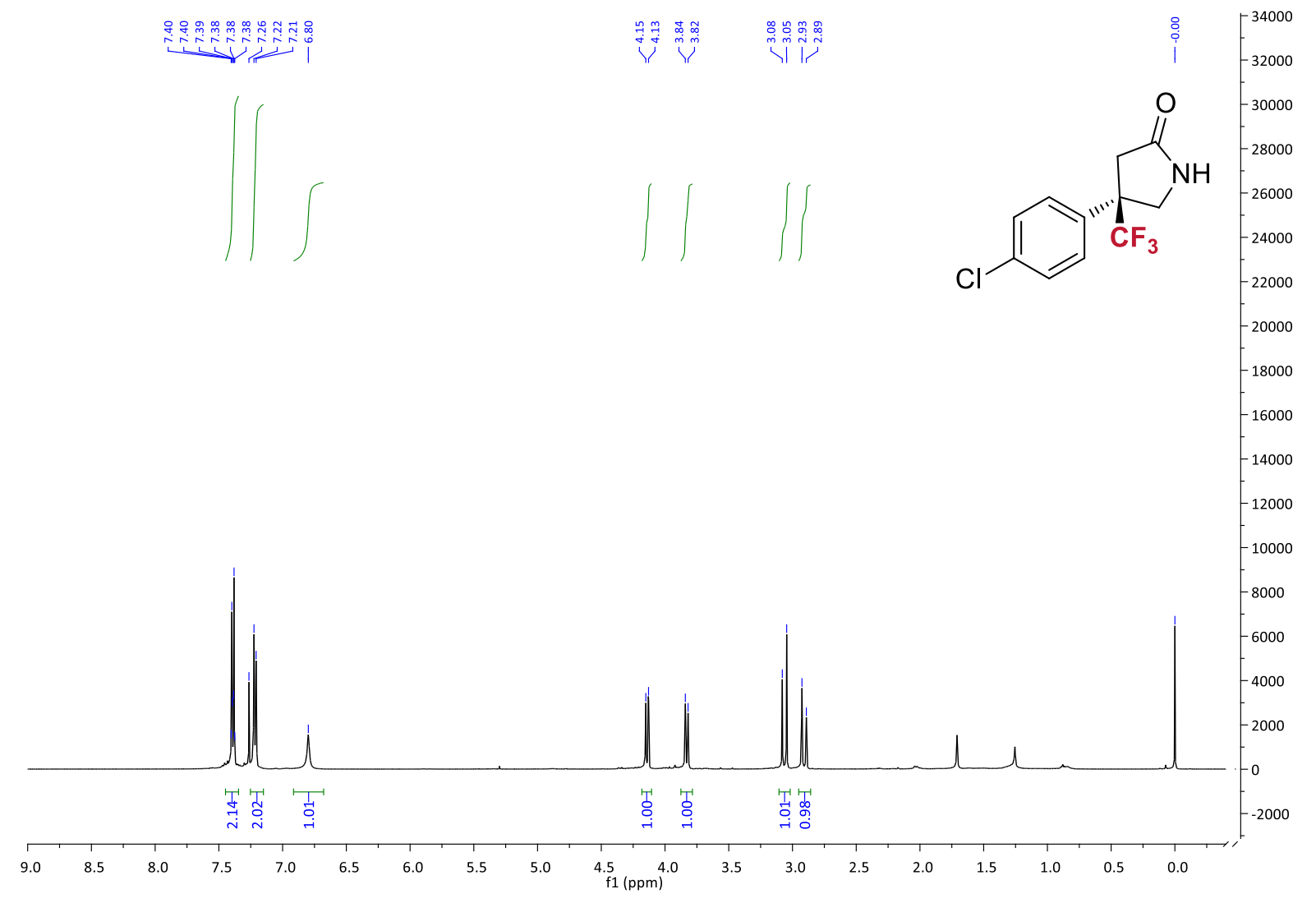

${ }^{1} \mathrm{H}$ NMR spectrum of $\mathbf{6} \mathbf{f}$

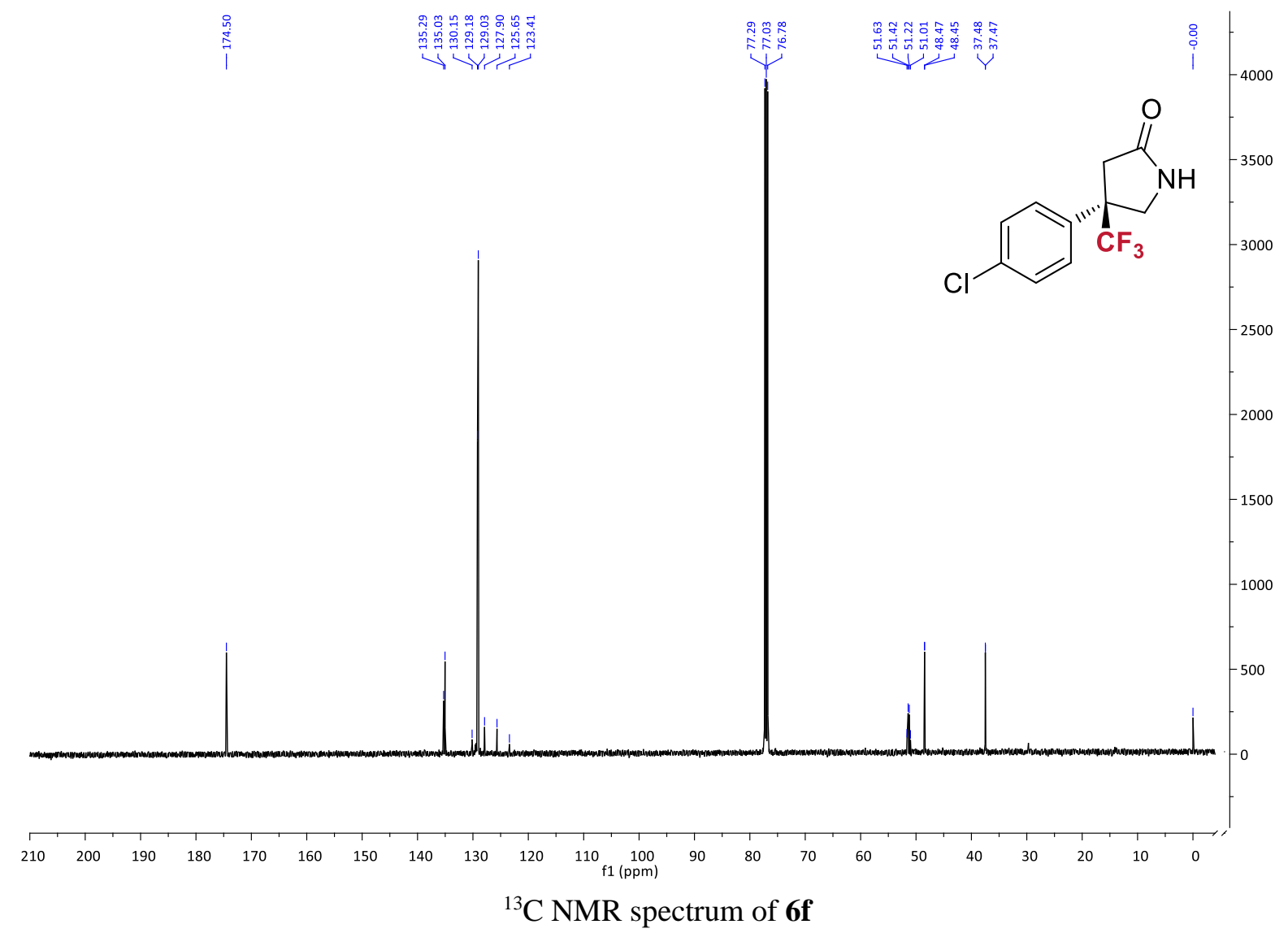




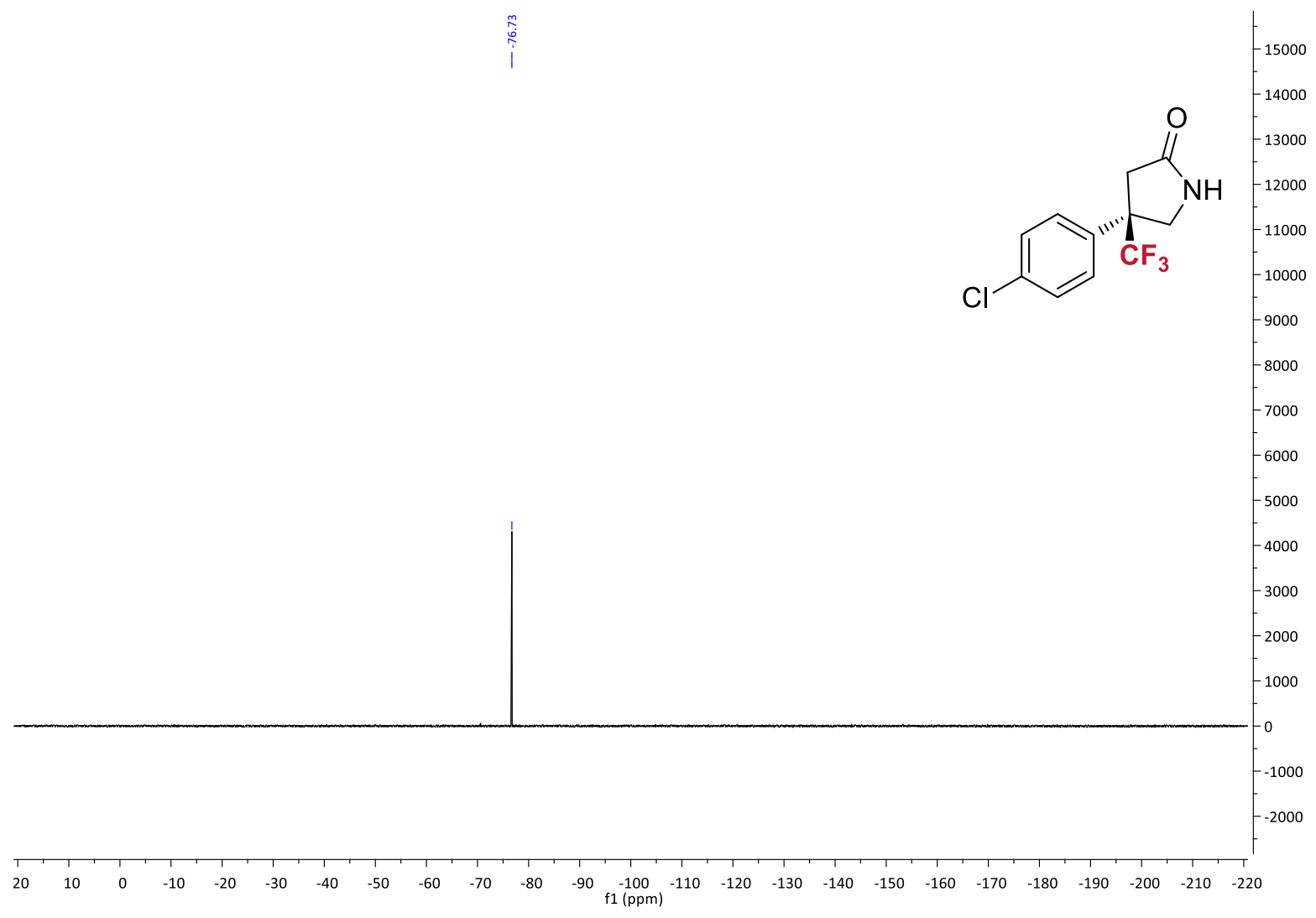

${ }^{19} \mathrm{~F}$ NMR spectrum of $\mathbf{6} \mathbf{f}$

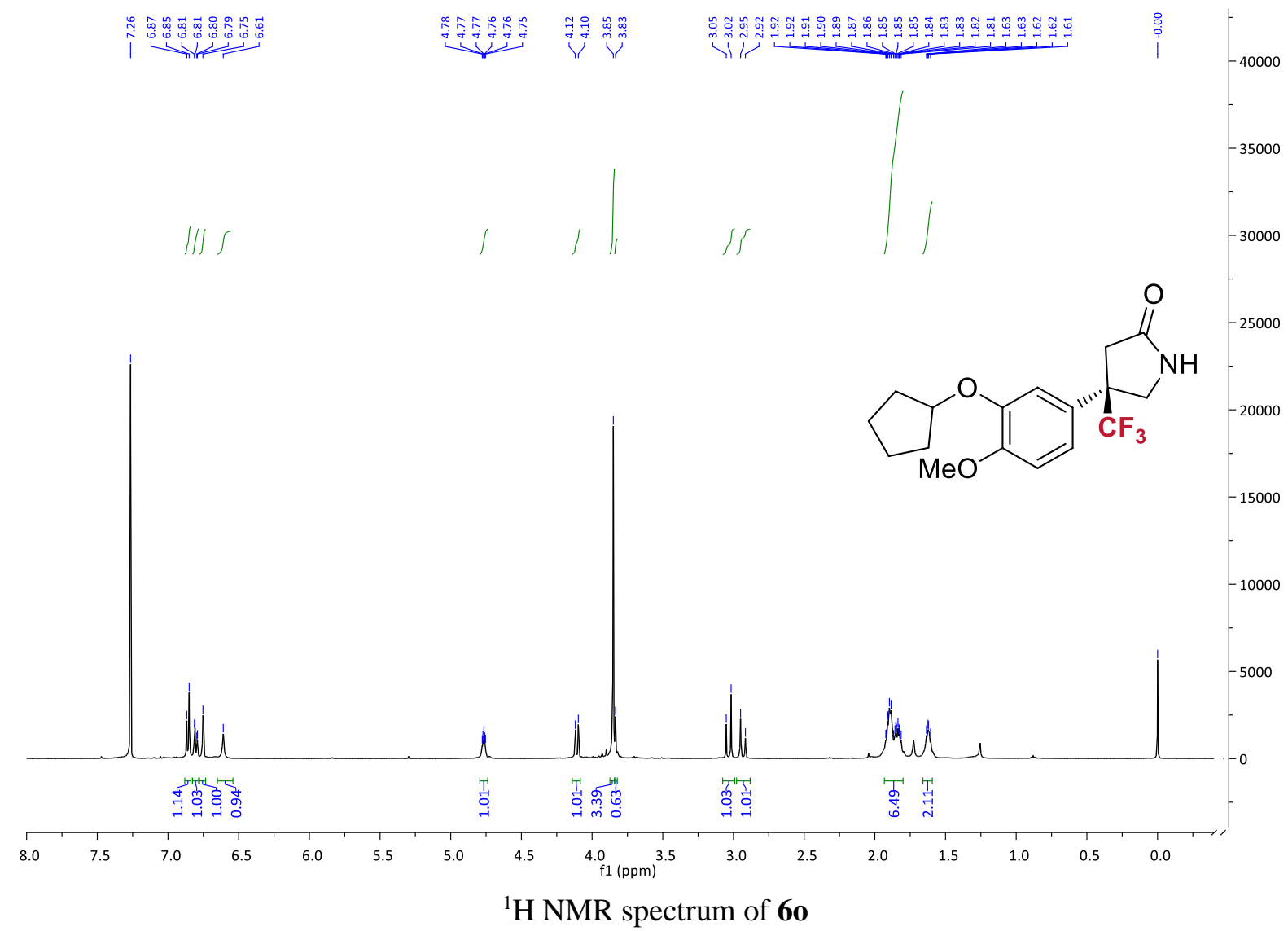




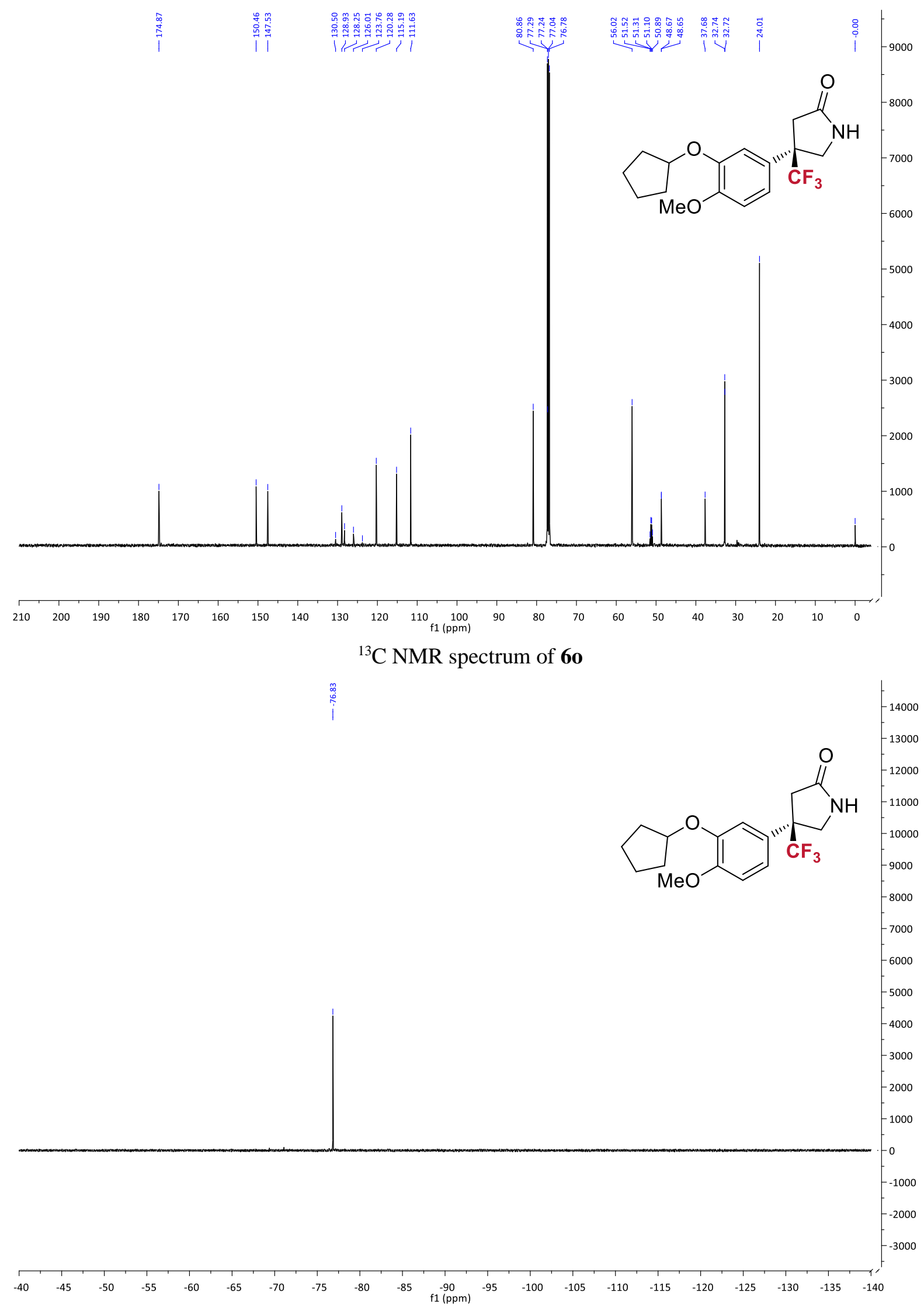

${ }^{19} \mathrm{~F}$ NMR spectrum of $\mathbf{6 o}$ 


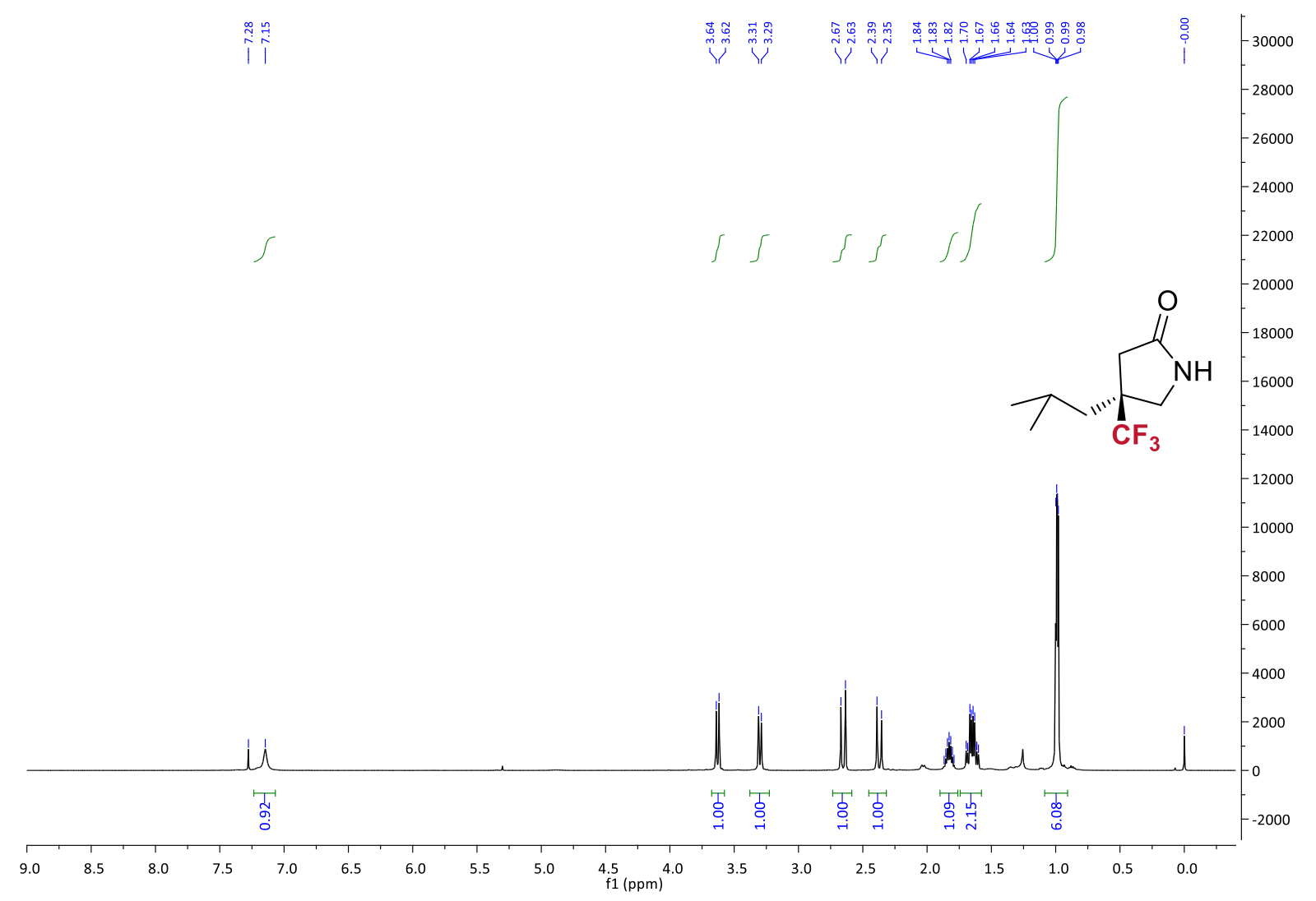

${ }^{1} \mathrm{H}$ NMR spectrum of $\mathbf{6 t}$

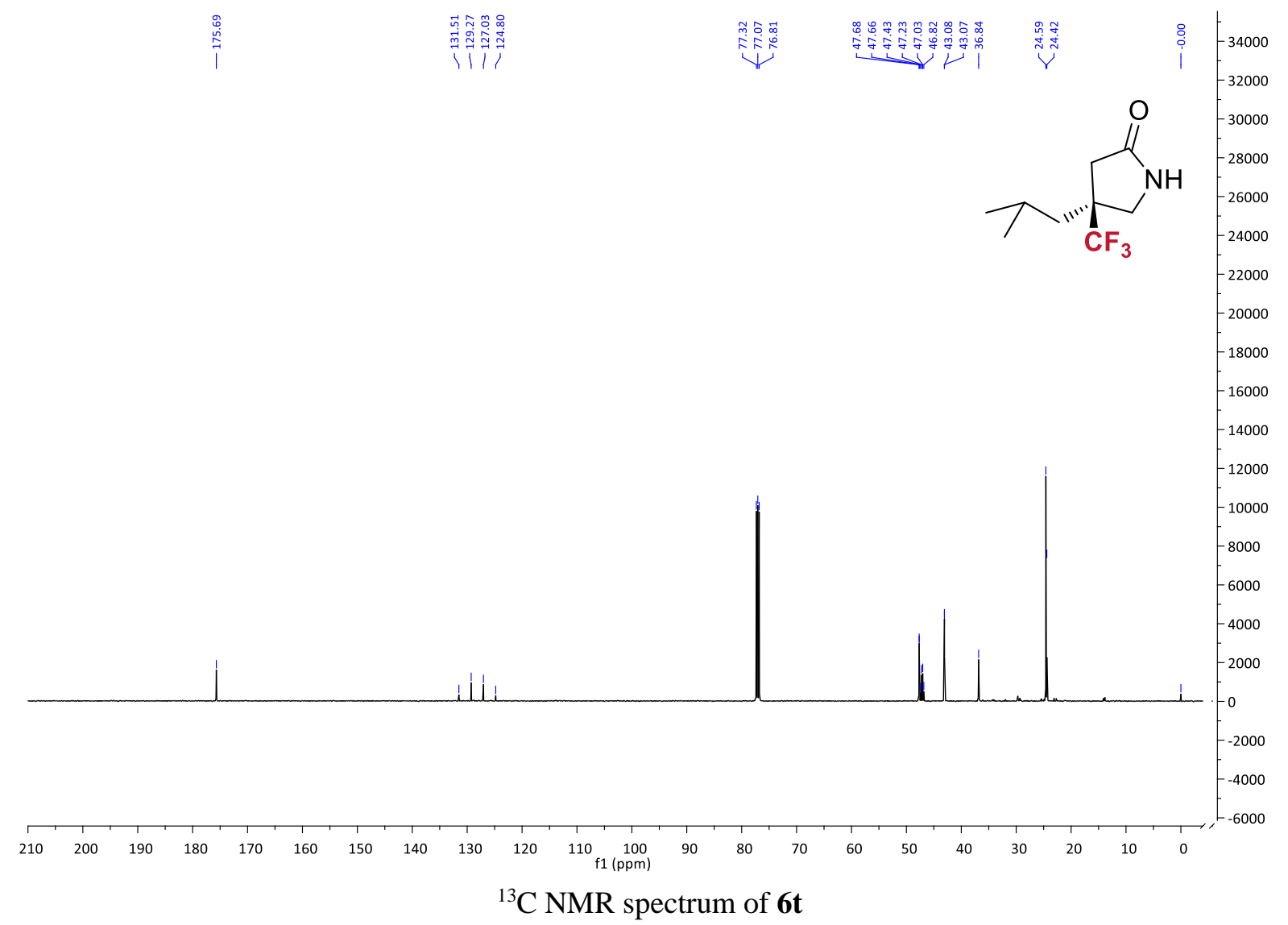




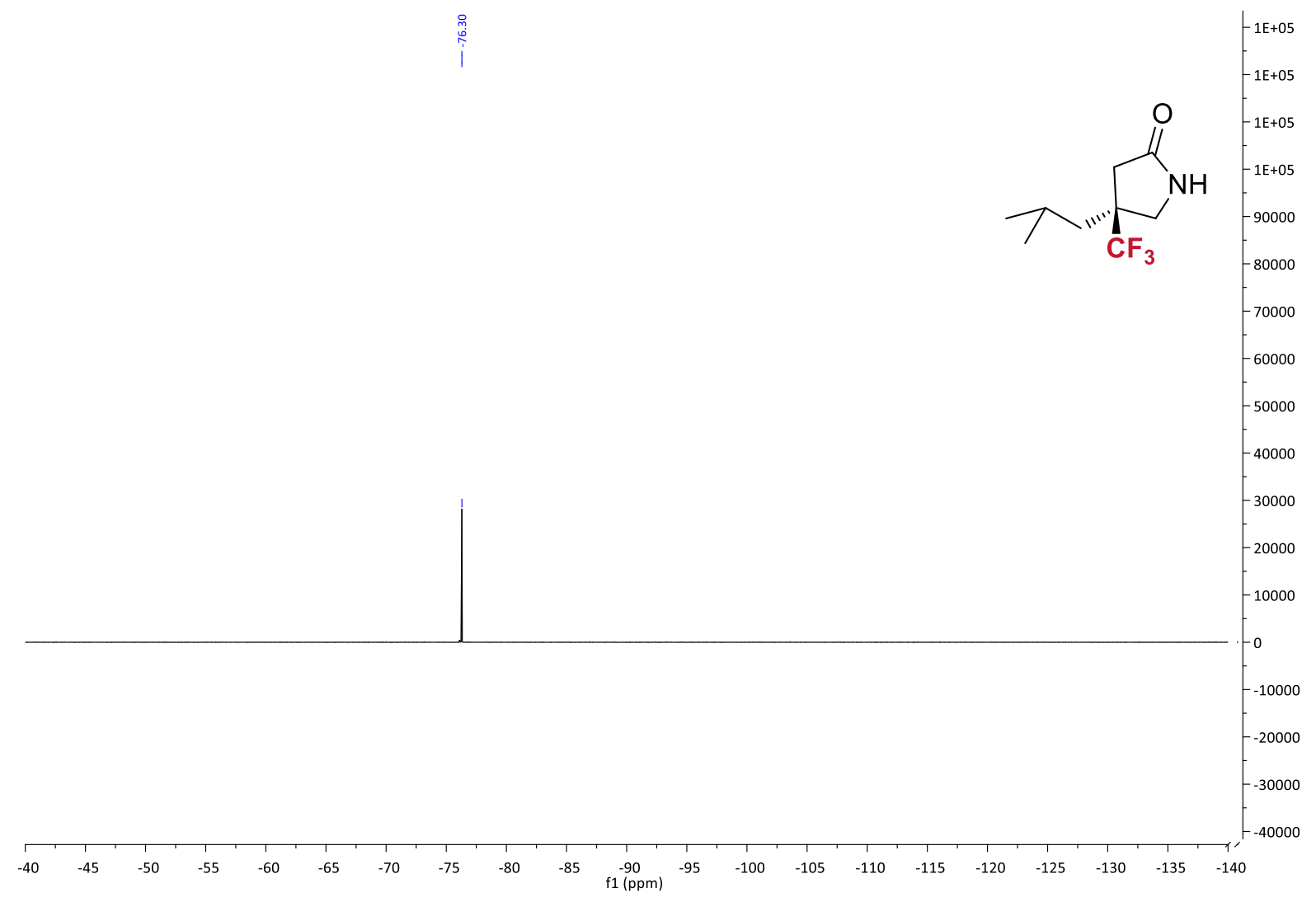

${ }^{19} \mathrm{~F}$ NMR spectrum of $\mathbf{6 t}$

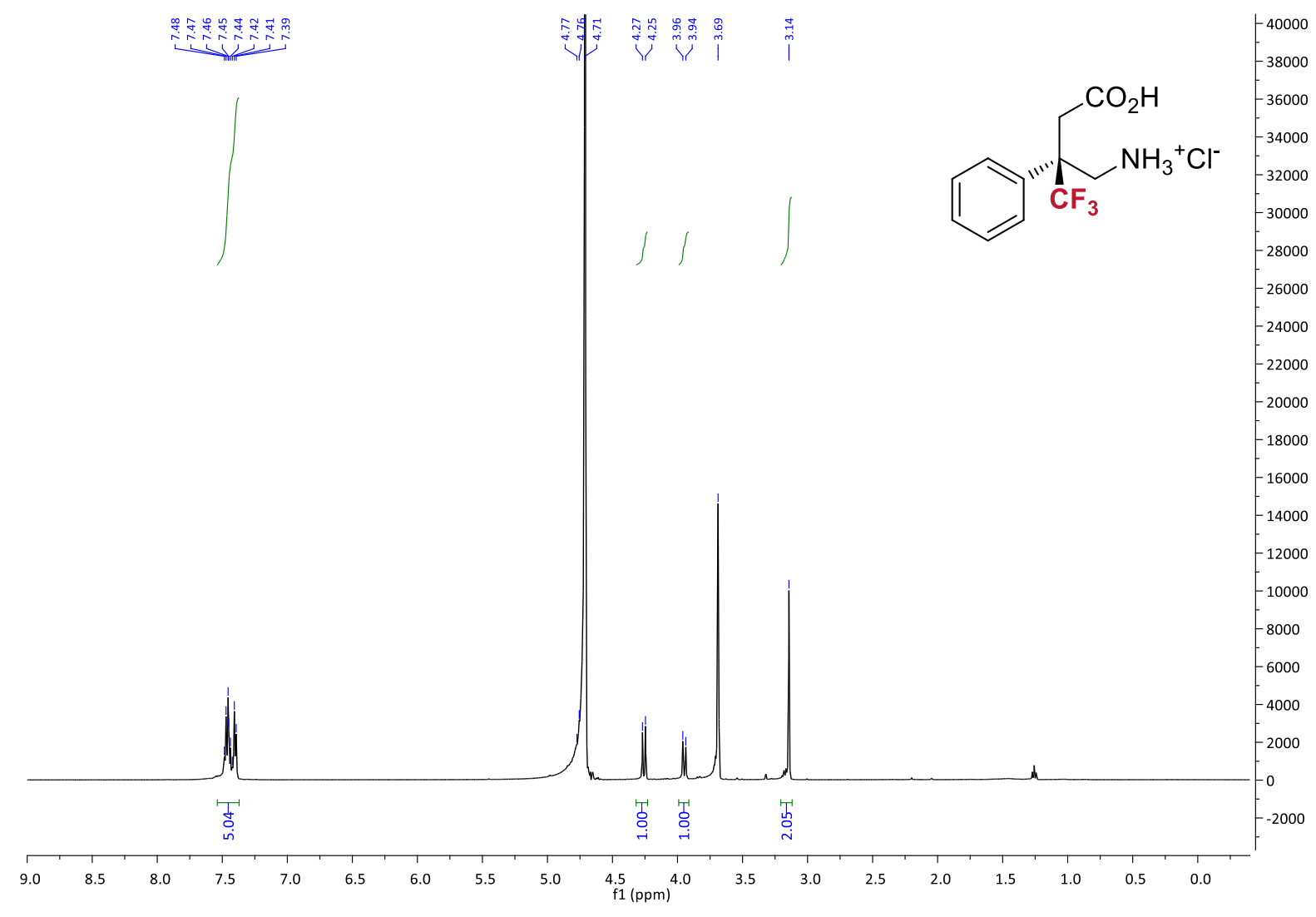

${ }^{1} \mathrm{H}$ NMR spectrum of $\mathbf{7 a}$ 


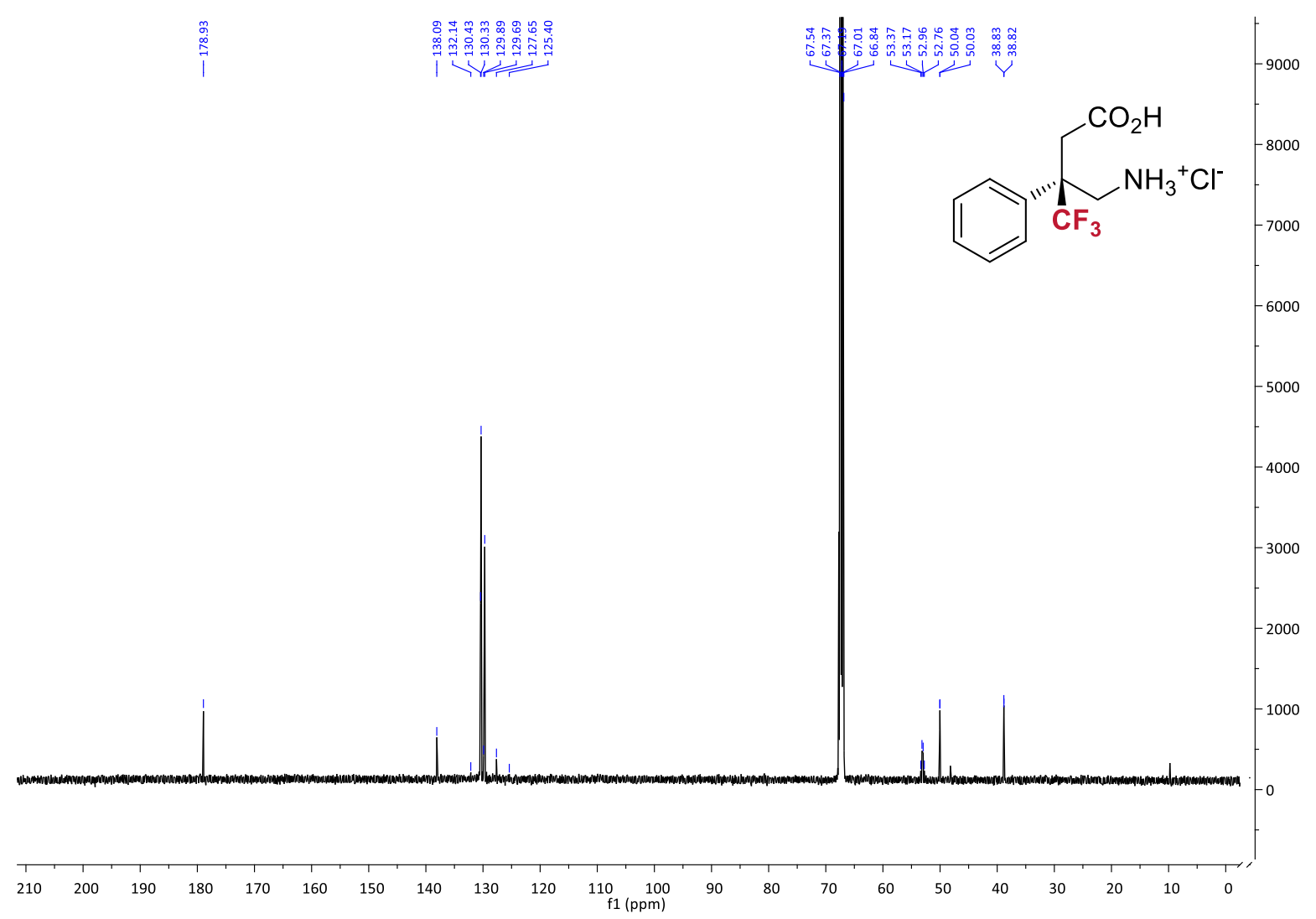

${ }^{13} \mathrm{C}$ NMR spectrum of $7 \mathbf{a}$

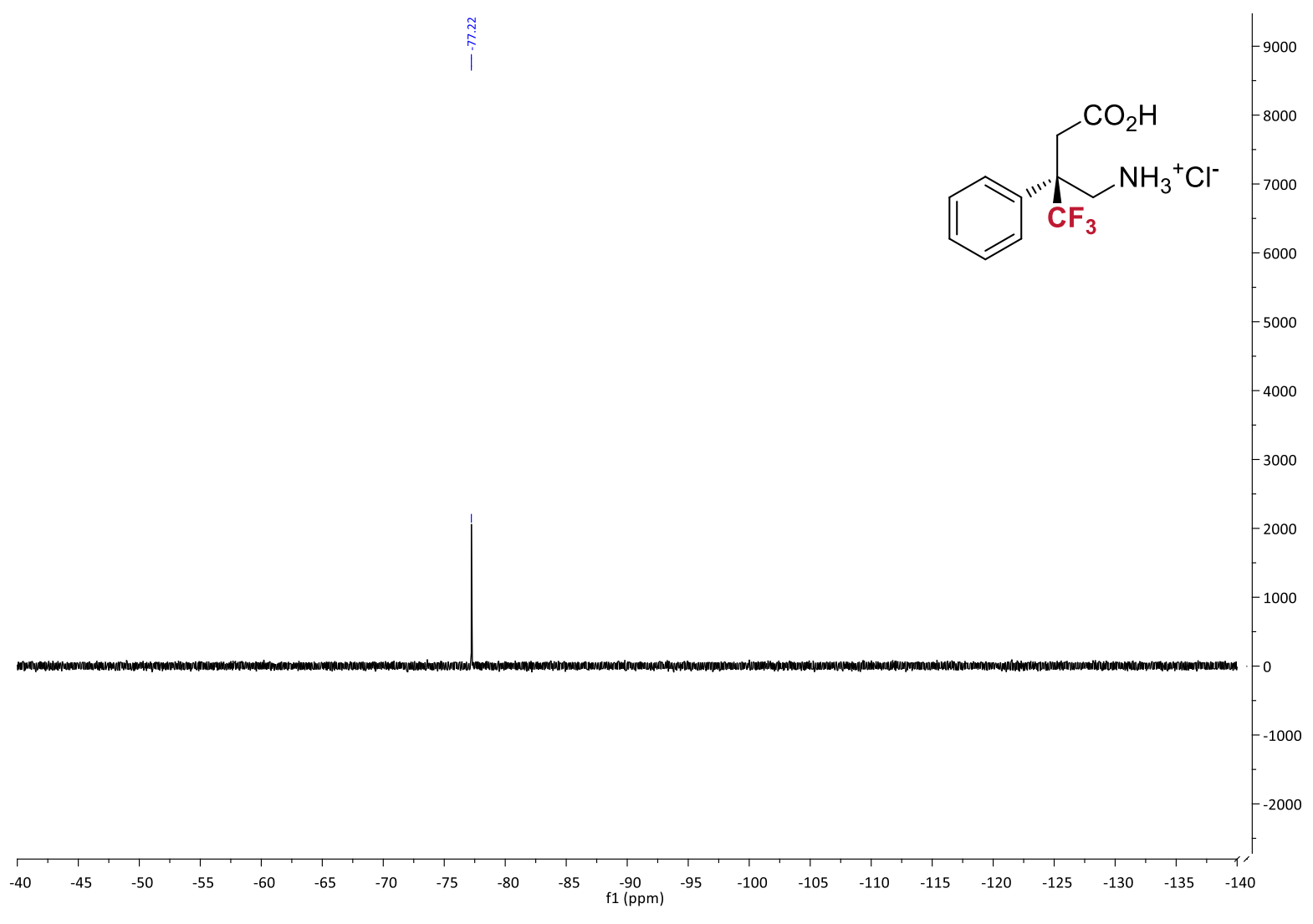

${ }^{19} \mathrm{~F}$ NMR spectrum of $\mathbf{7 a}$ 


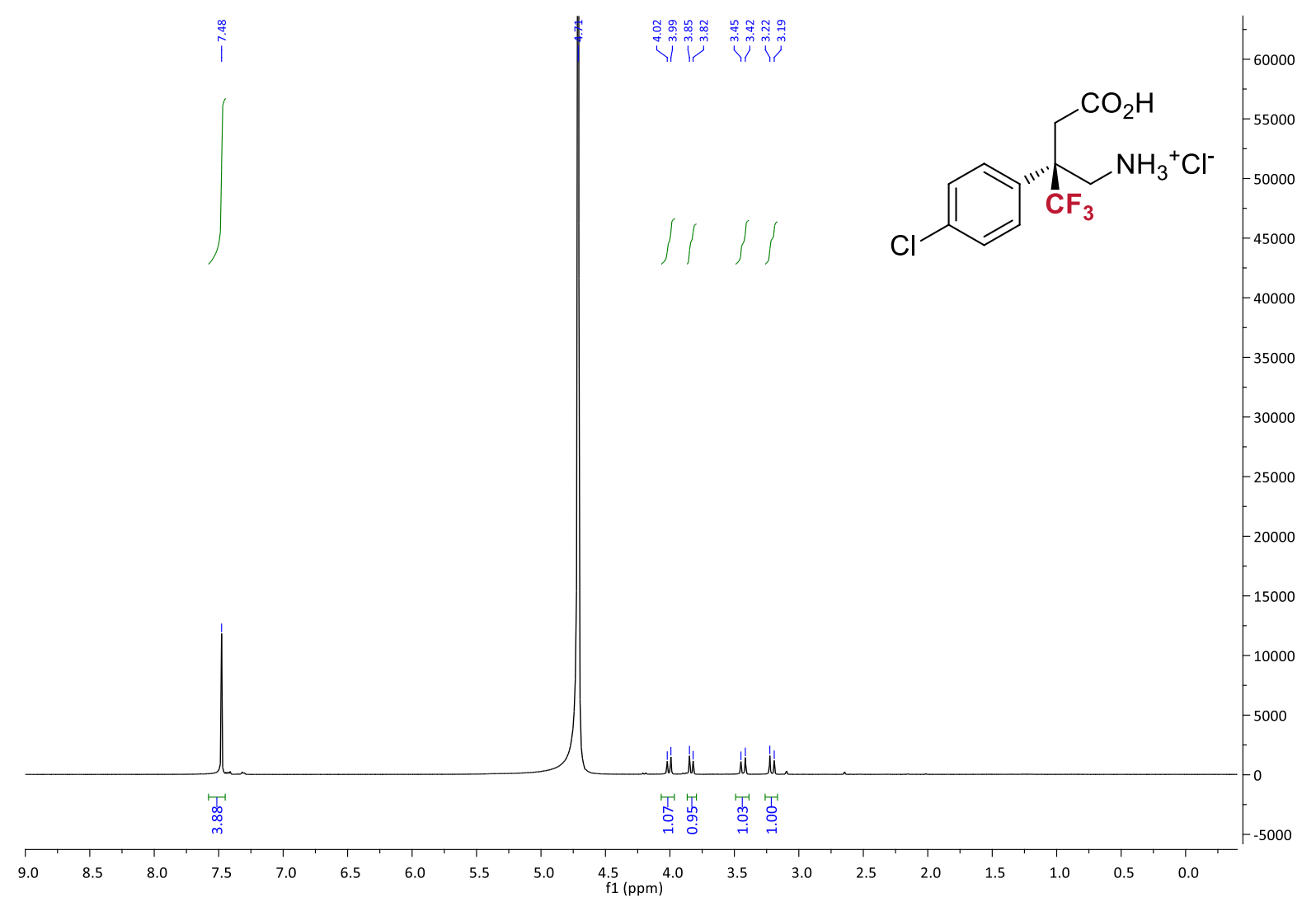

${ }^{1} \mathrm{H}$ NMR spectrum of $7 \mathbf{f}$

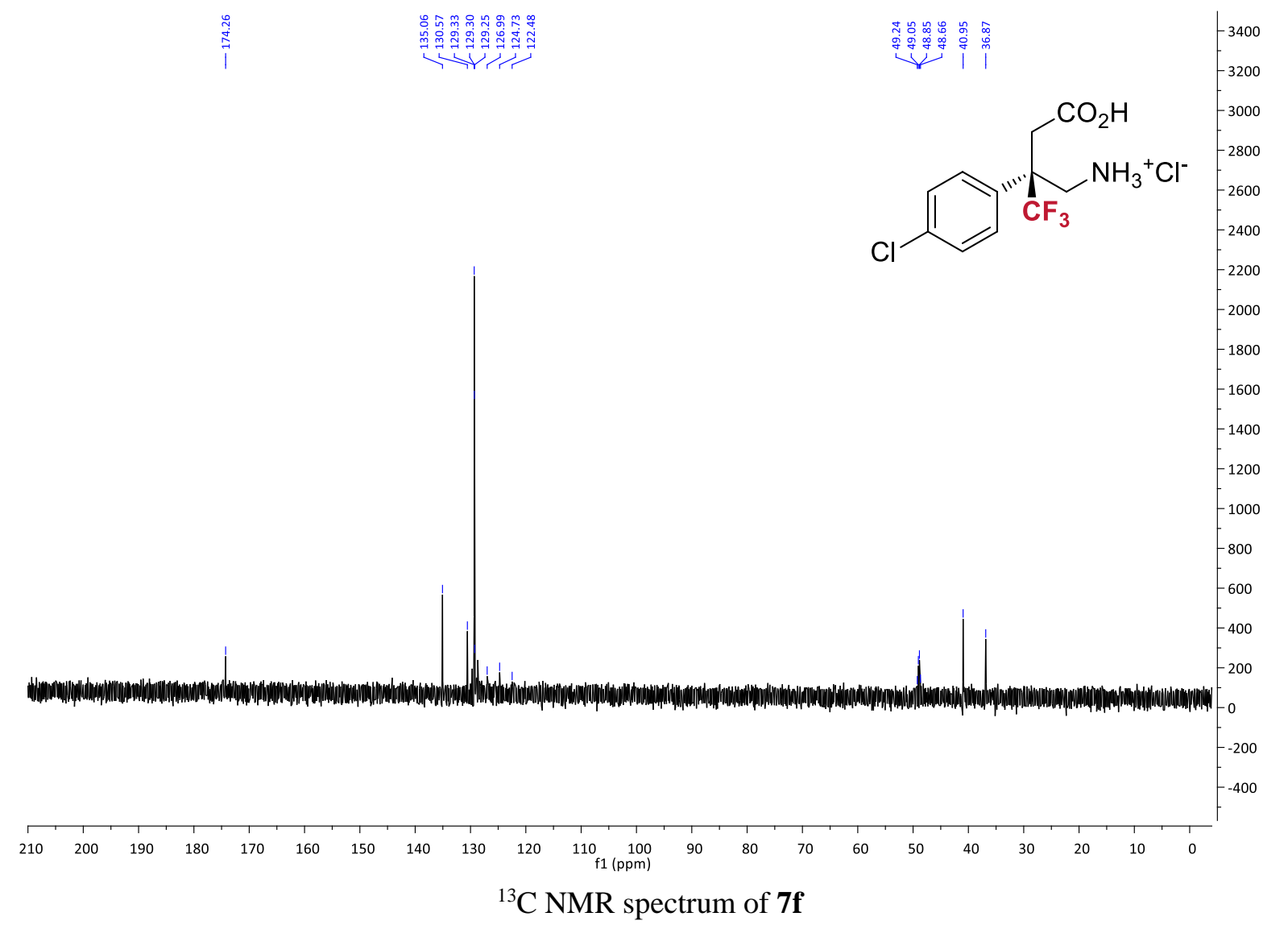




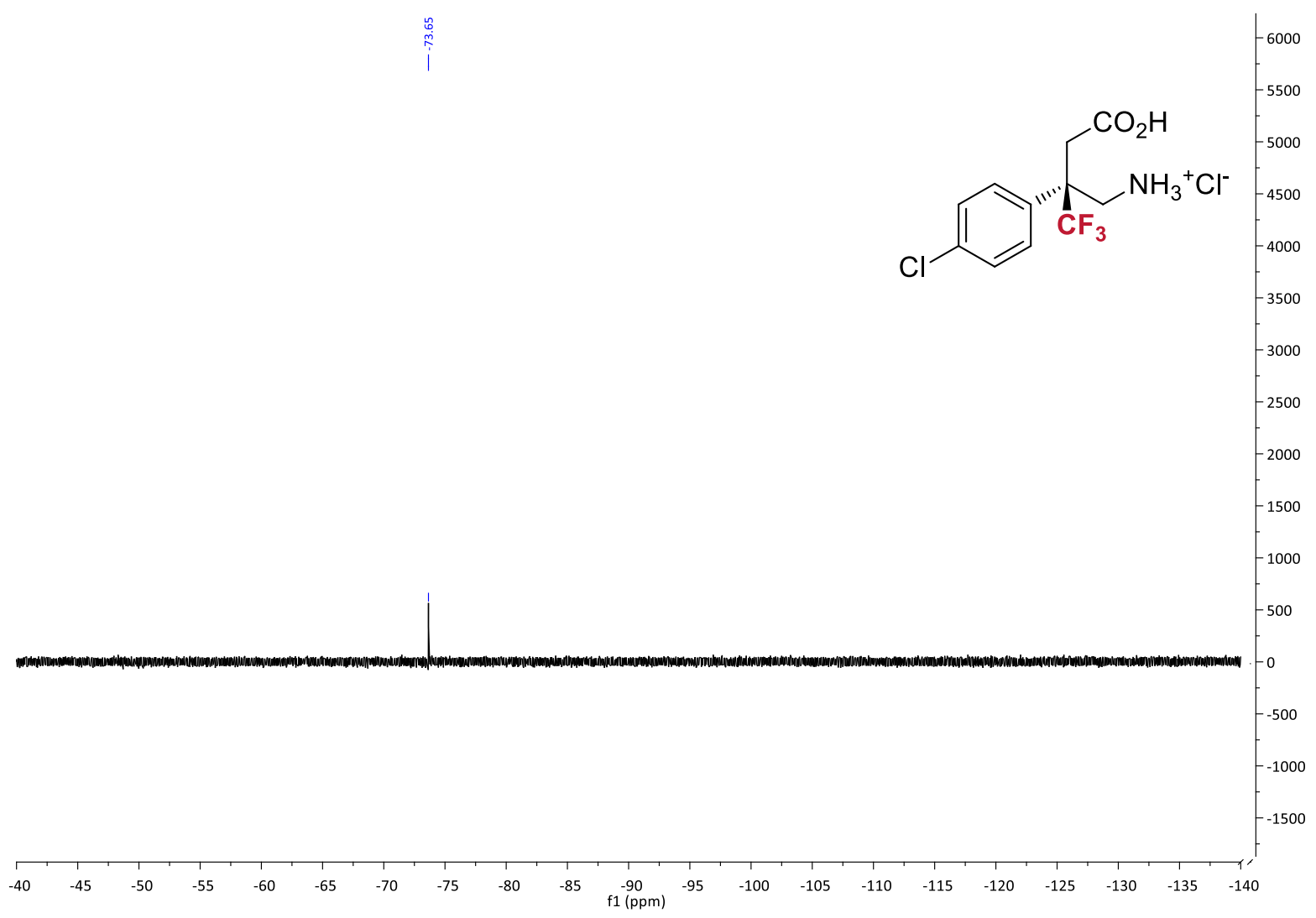

${ }^{19} \mathrm{~F}$ NMR spectrum of $\mathbf{7 f}$

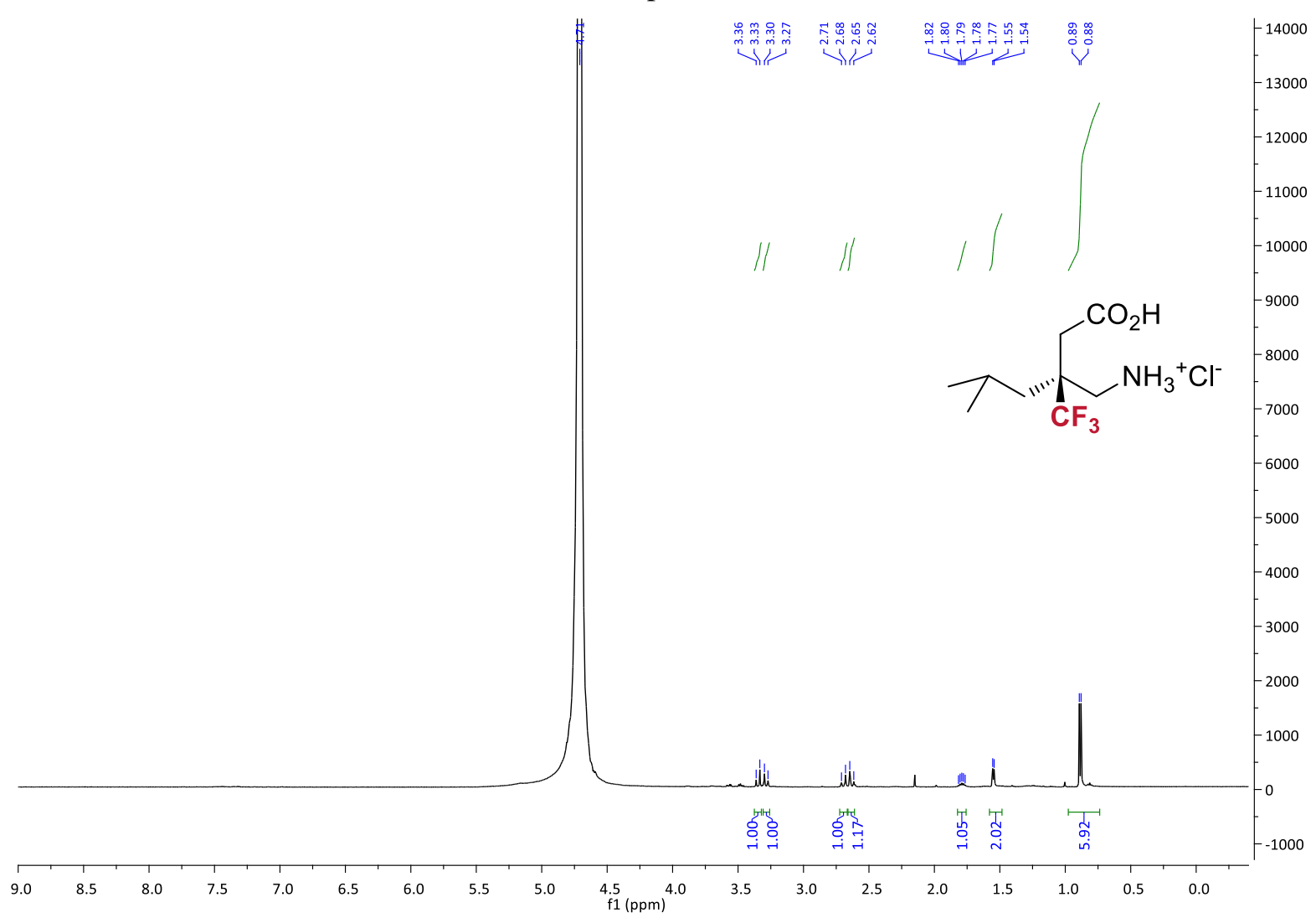

${ }^{1} \mathrm{H}$ NMR spectrum of $\mathbf{7 t}$ 


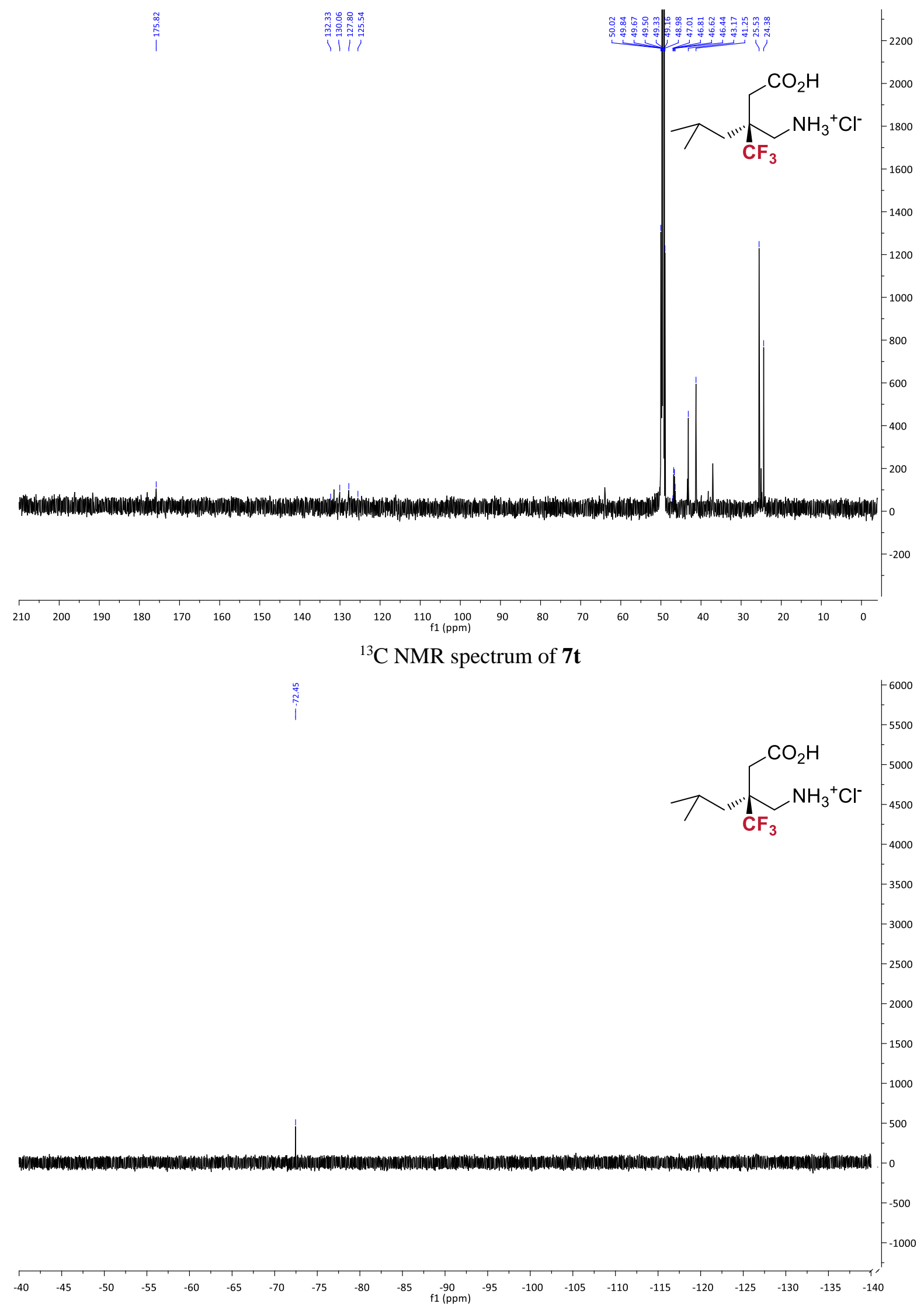

${ }^{19} \mathrm{~F}$ NMR spectrum of $\mathbf{7 t}$ 
6.2. HPLC spectra of the products $(3-7)$

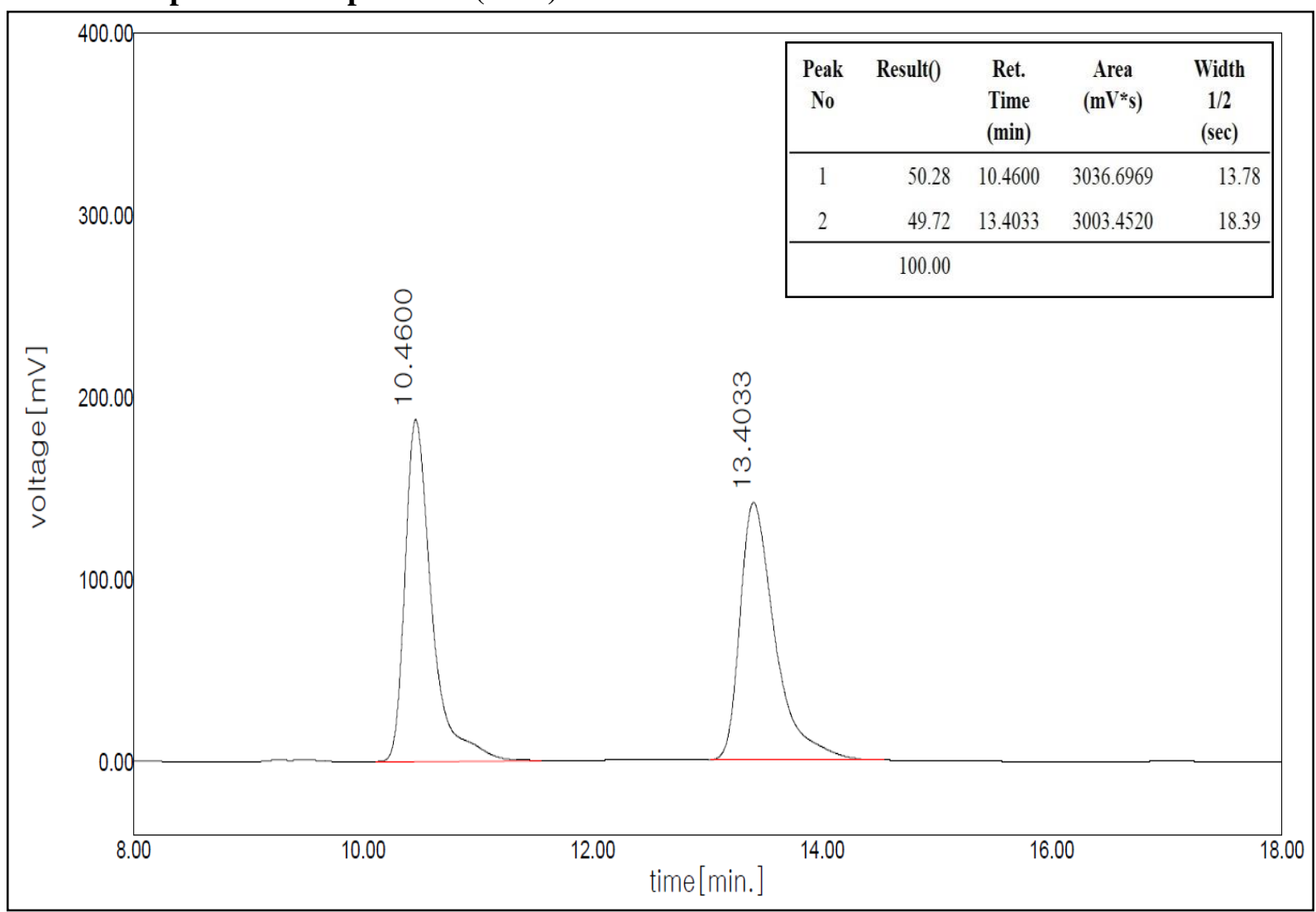

HPLC spectrum of rac-3ab

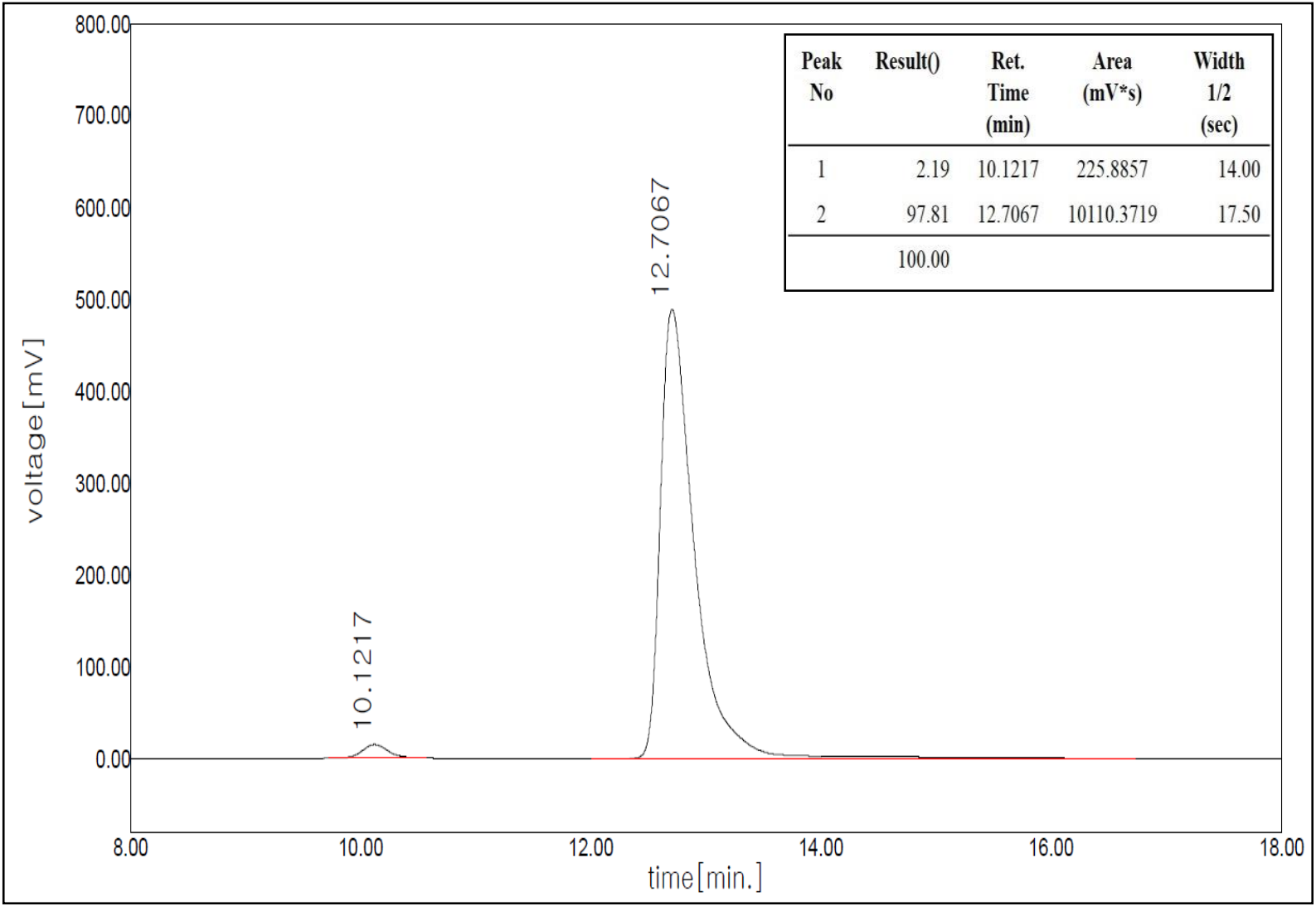

HPLC spectrum of $\mathbf{3 a b}$ 


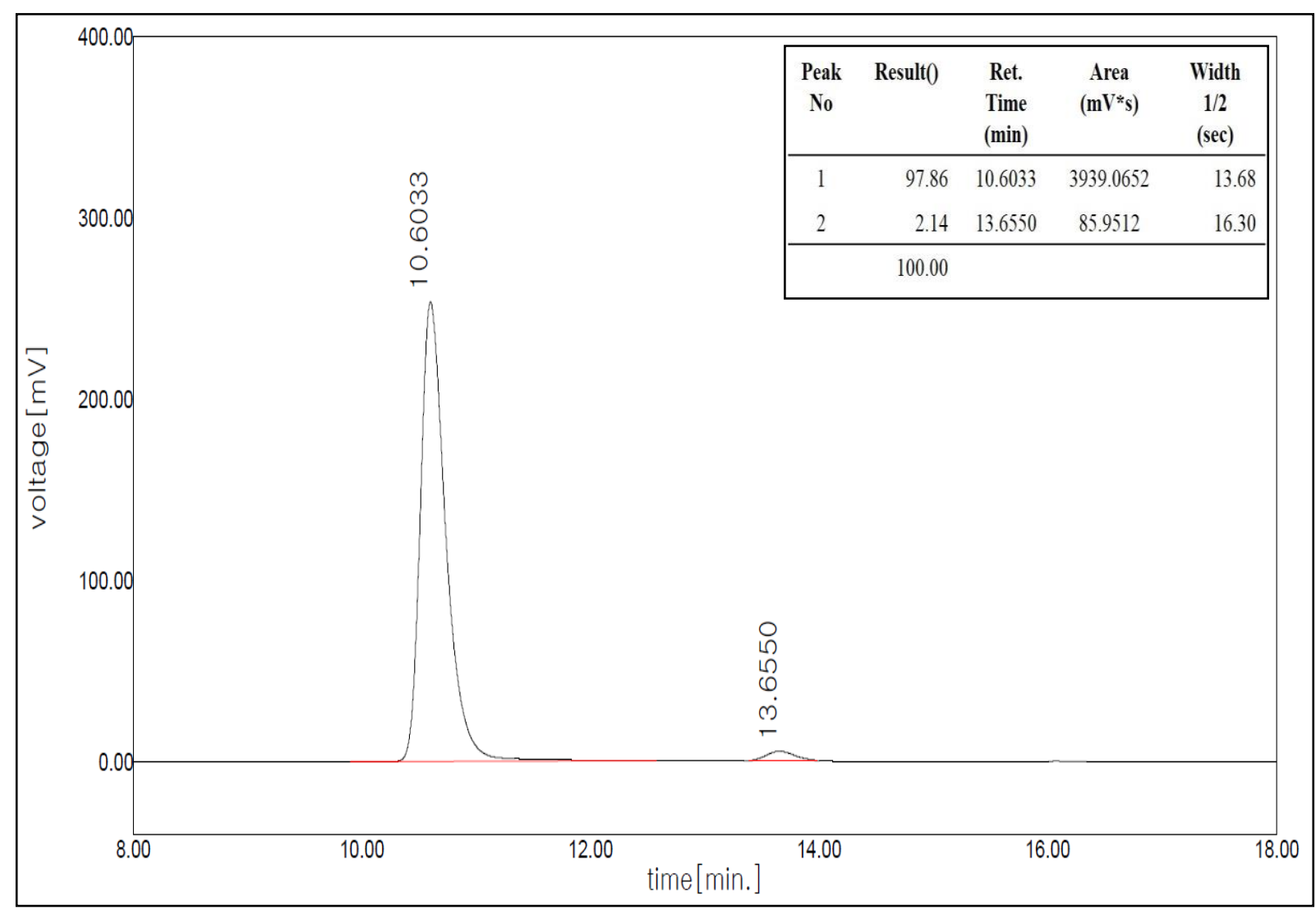

HPLC spectrum of ent-3ab

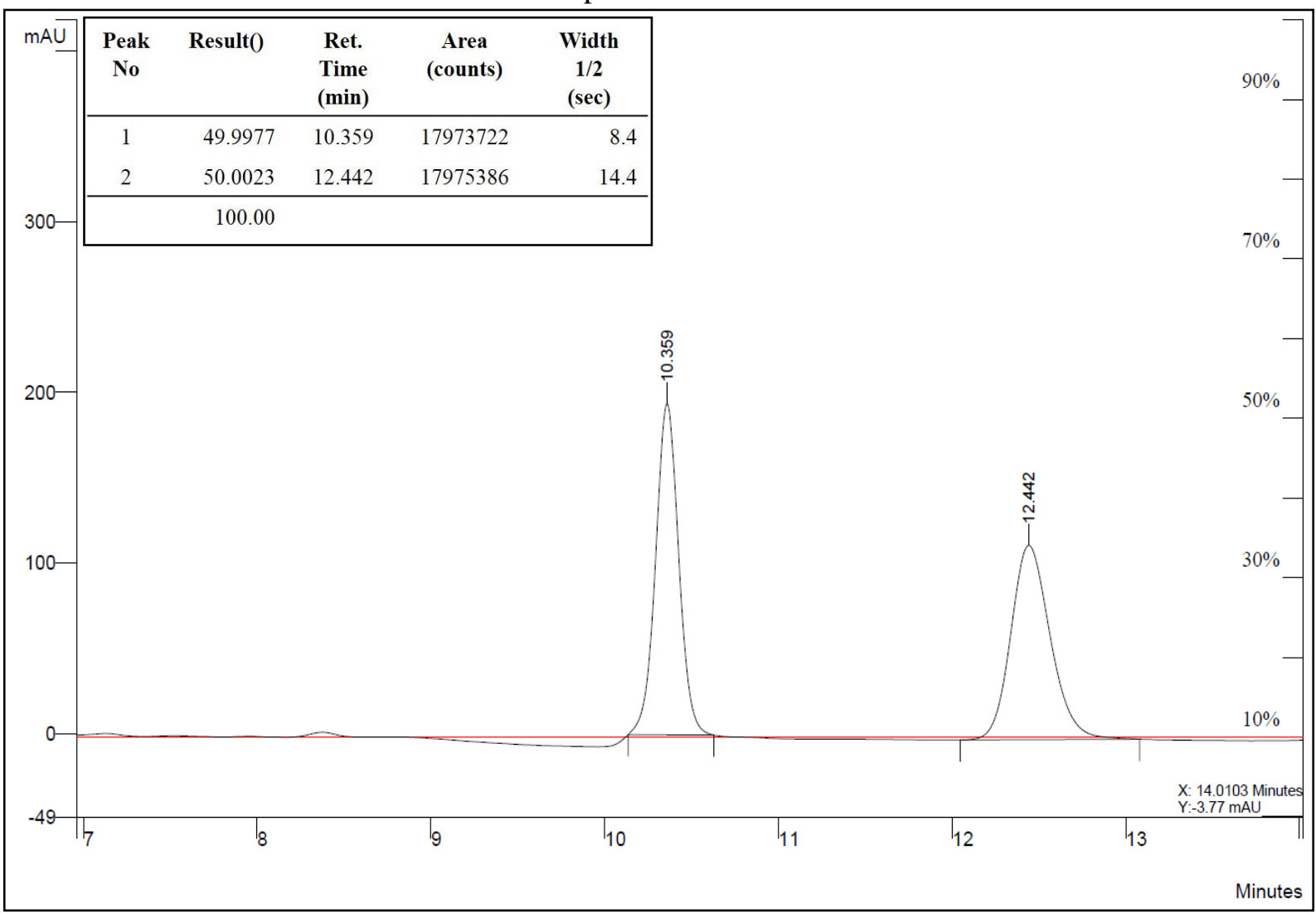

HPLC spectrum of $r a c-\mathbf{3 b}$ 


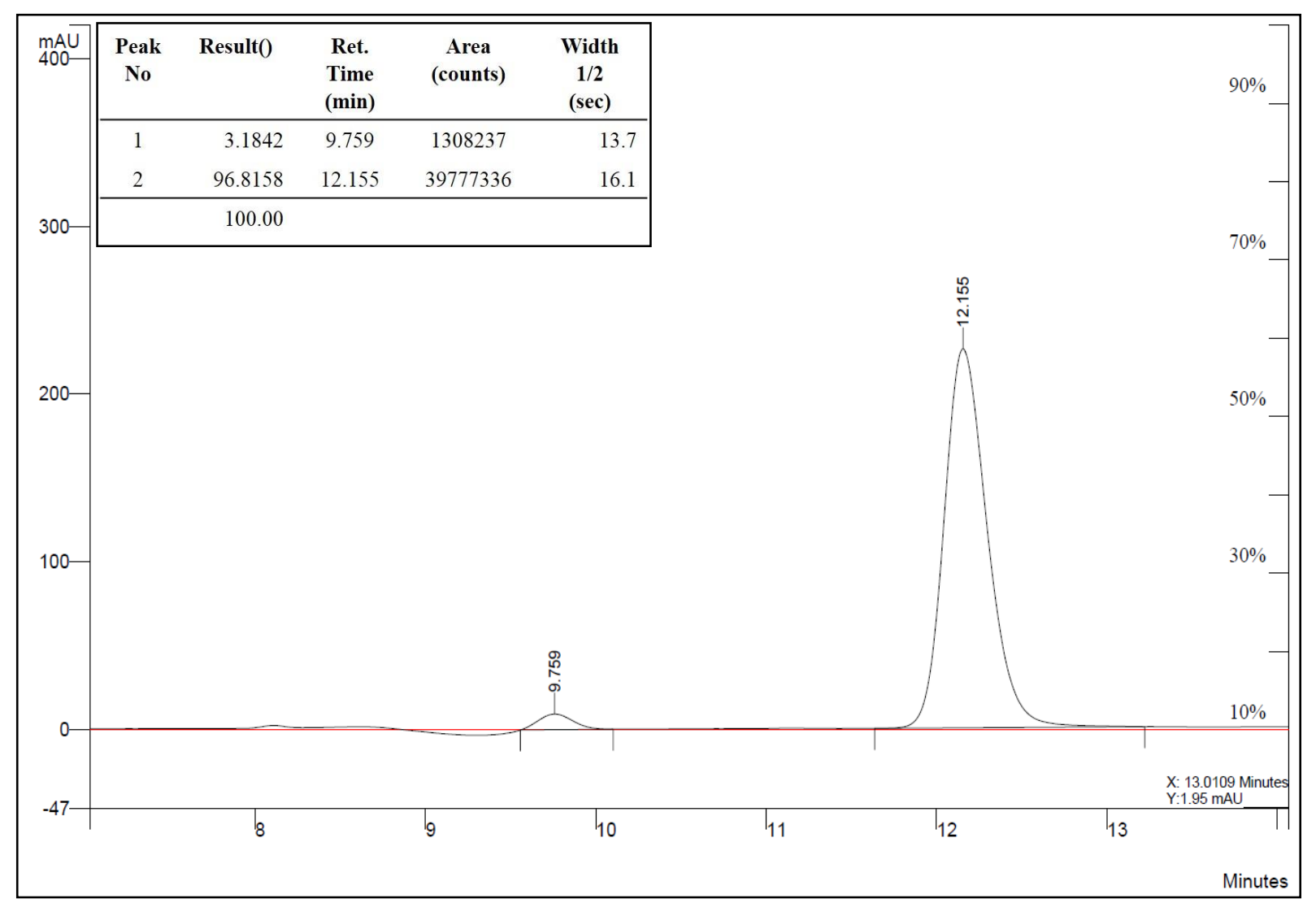

HPLC spectrum of $\mathbf{3 b}$

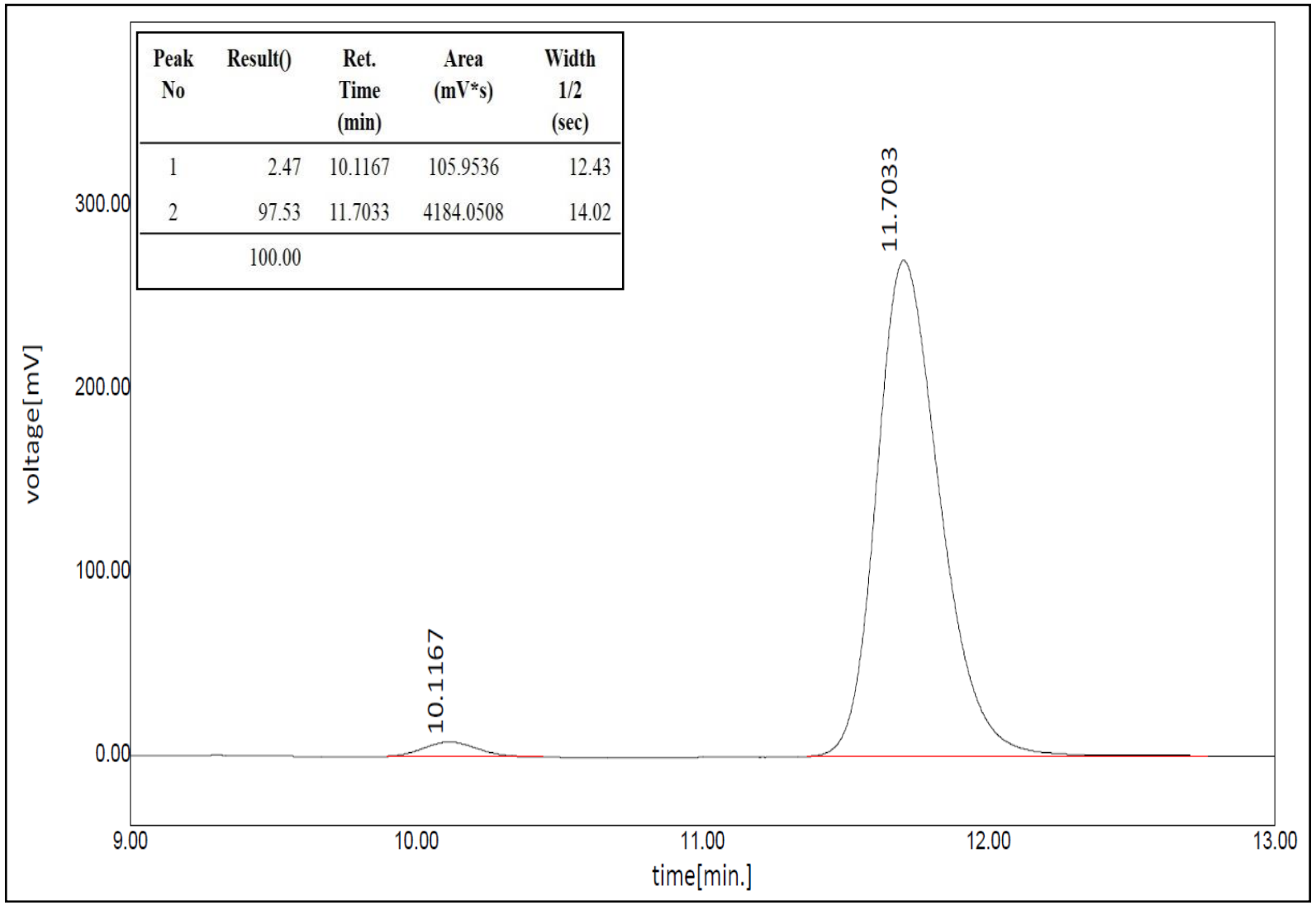

HPLC spectrum of $\mathbf{3 c}$ 


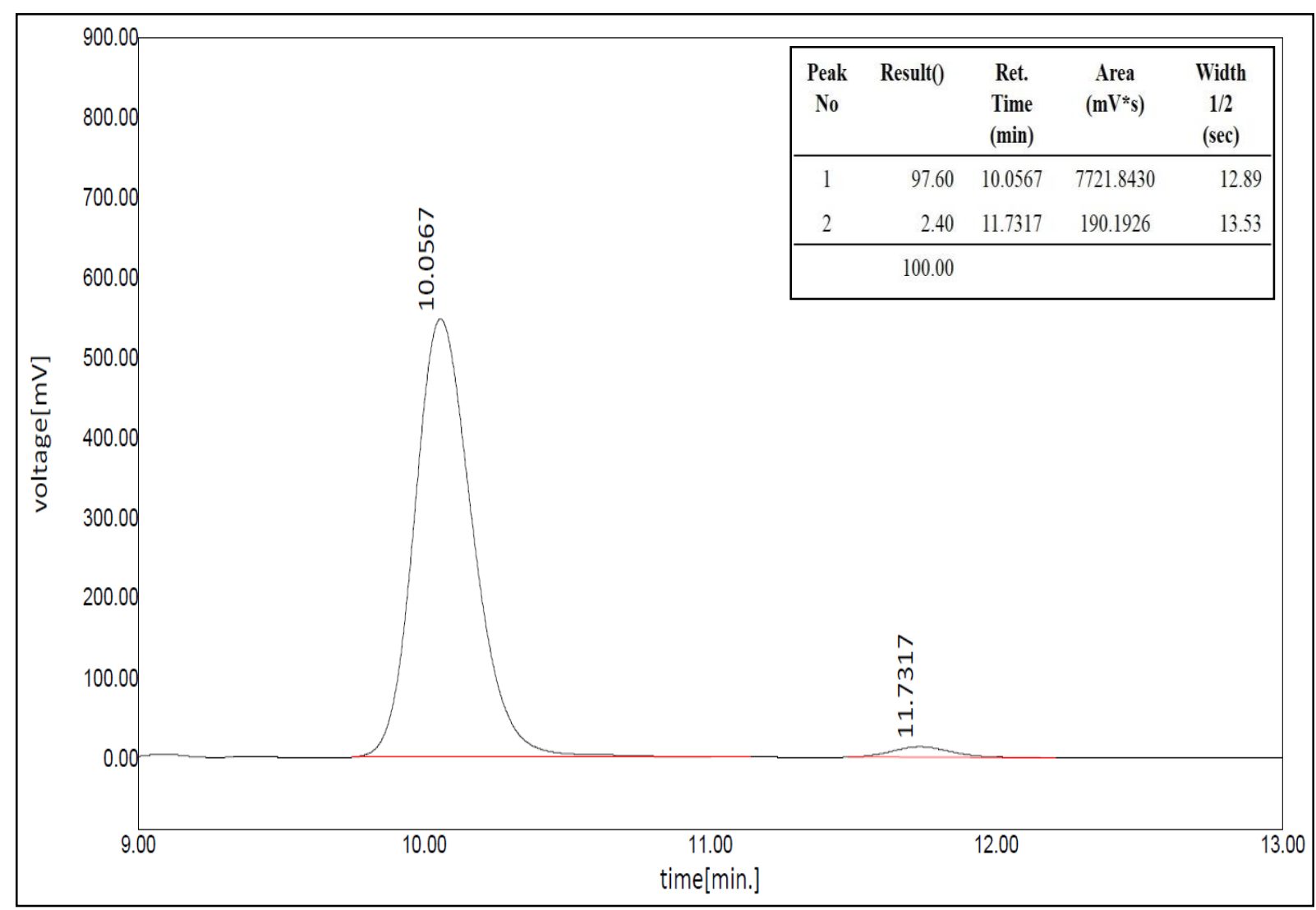

HPLC spectrum of ent-3c

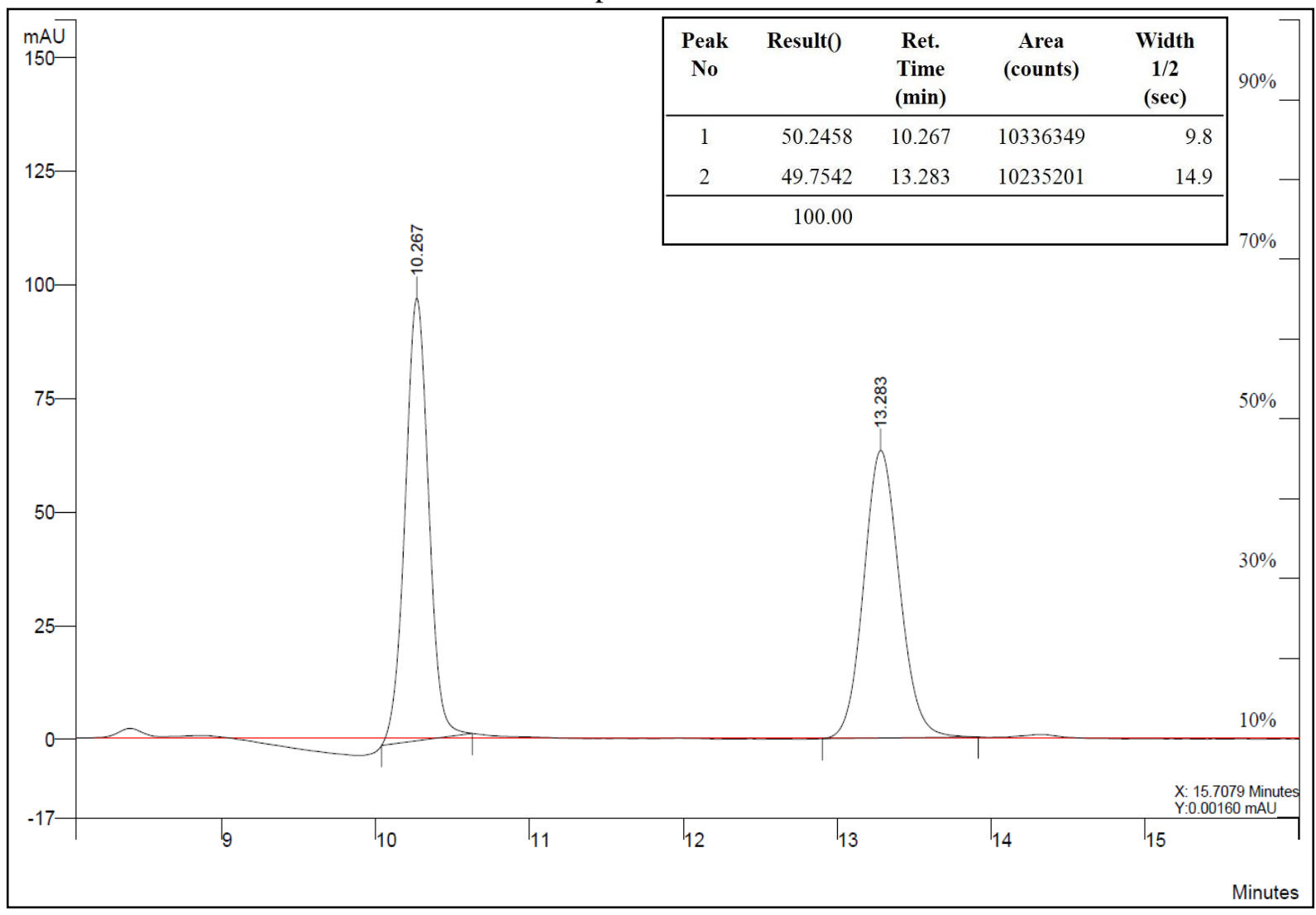

HPLC spectrum of $r a c-\mathbf{3 d}$ 


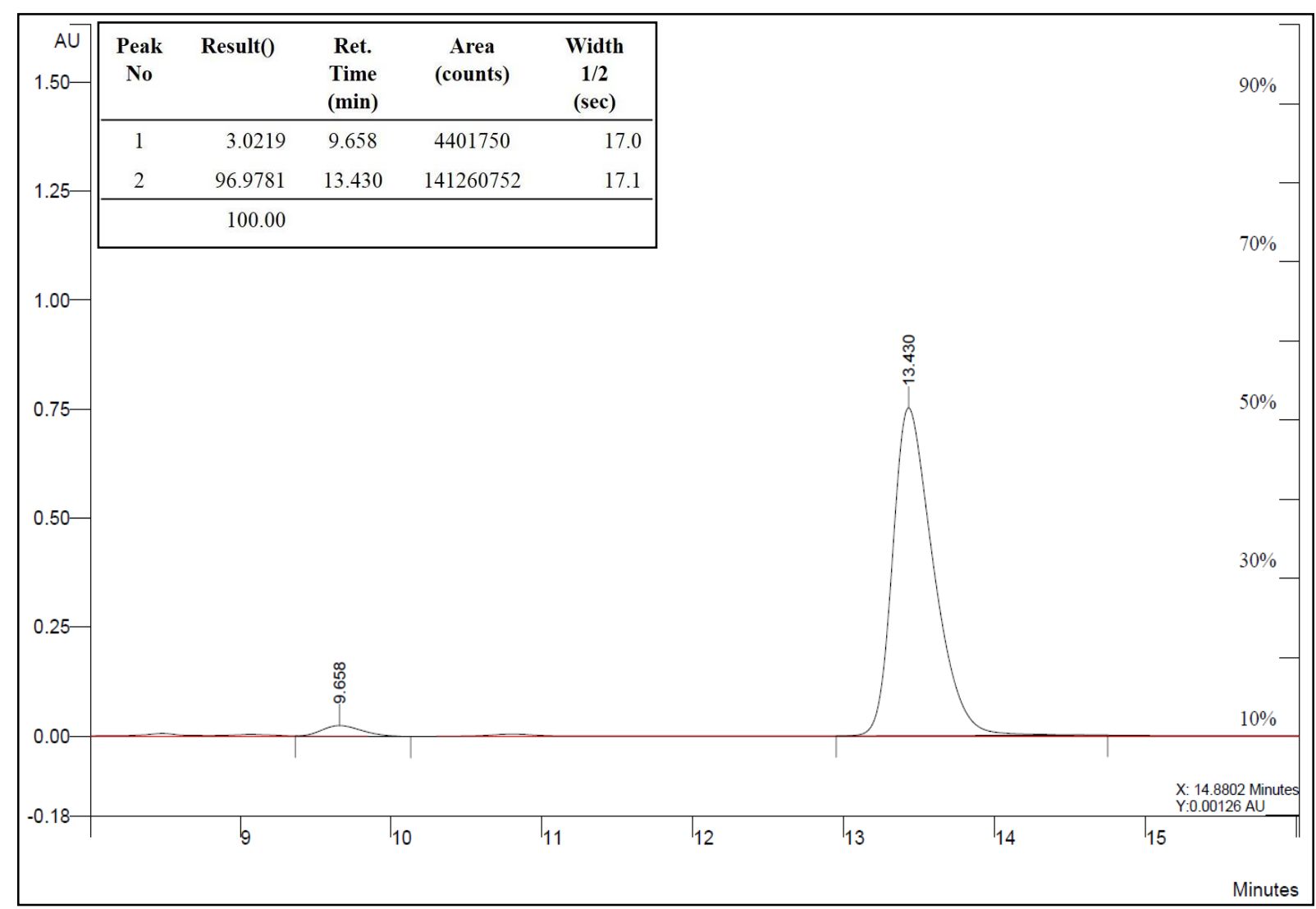

HPLC spectrum of $\mathbf{3 d}$

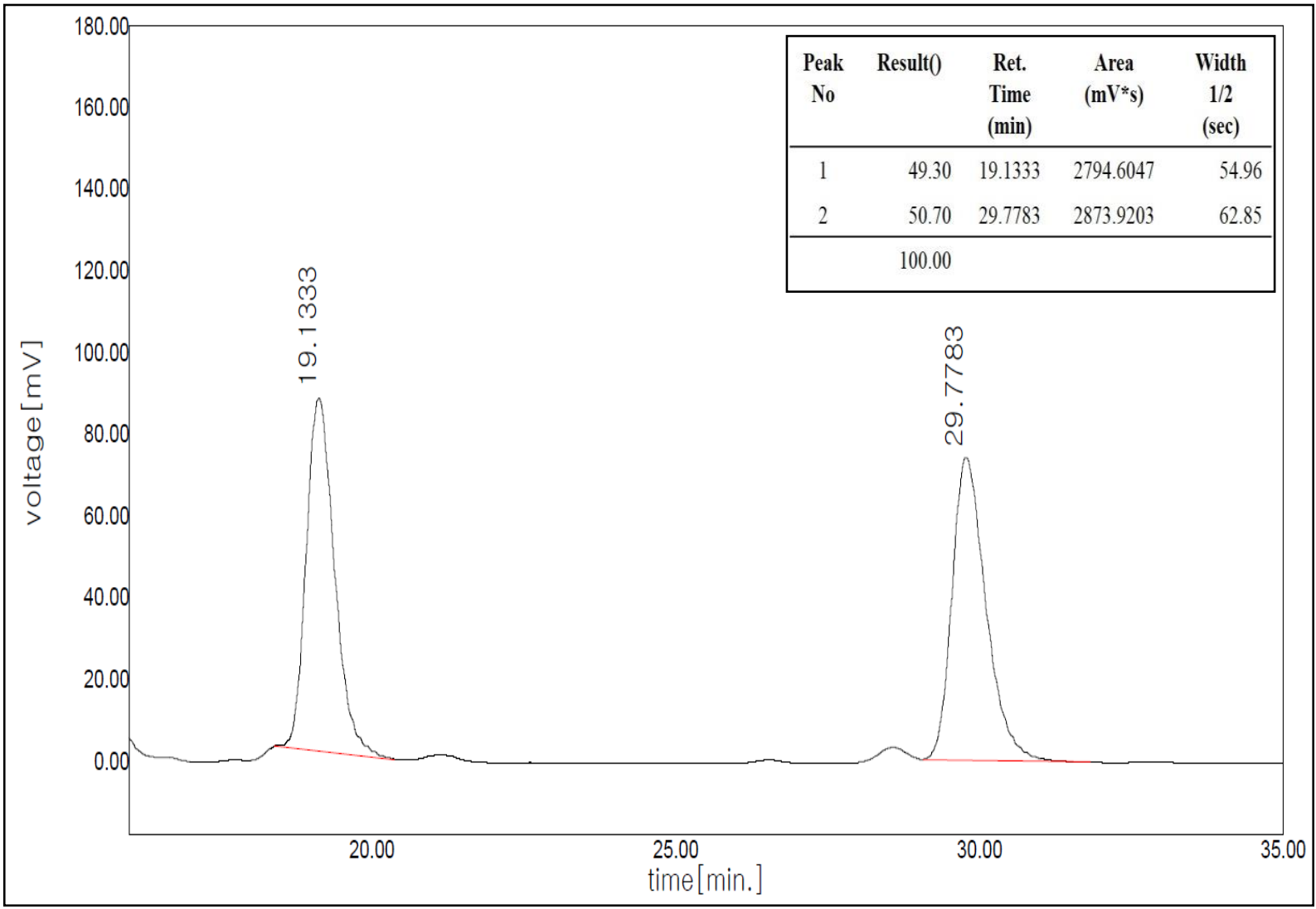

HPLC spectrum of $\mathrm{rac}-\mathbf{3 e}$ 


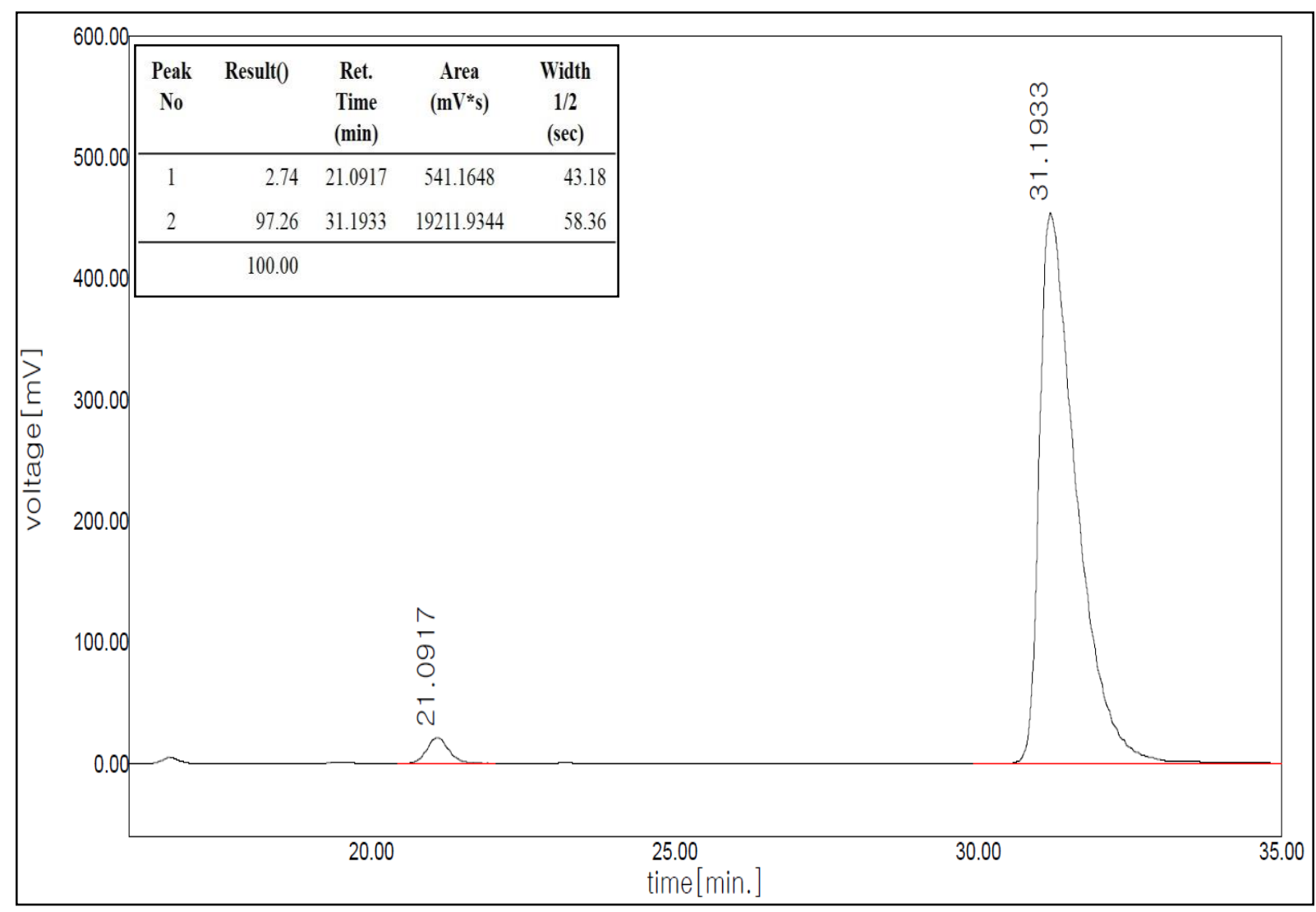

HPLC spectrum of $\mathbf{3 e}$

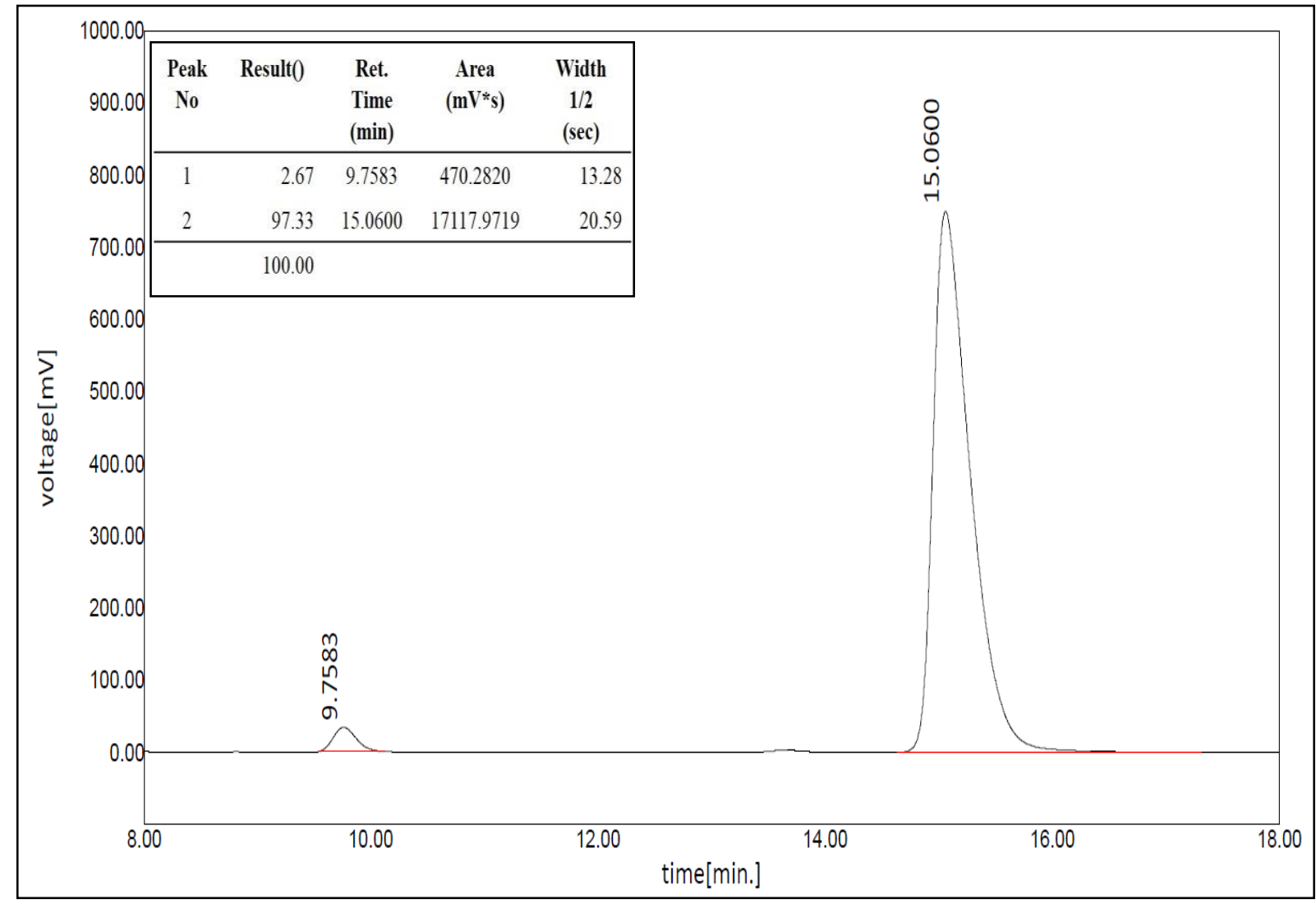

HPLC spectrum of $\mathbf{3 f}$ 


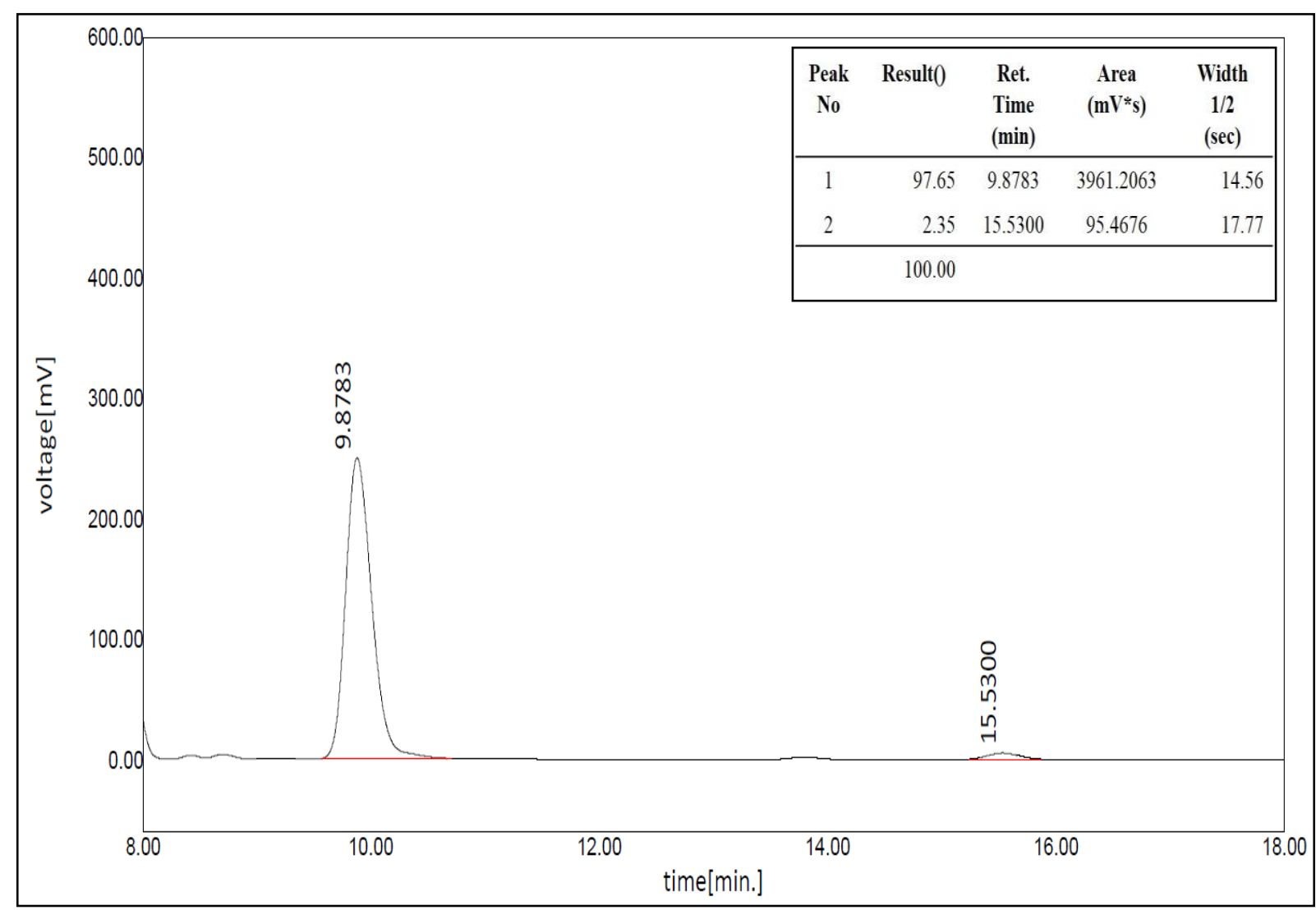

HPLC spectrum of ent-3f

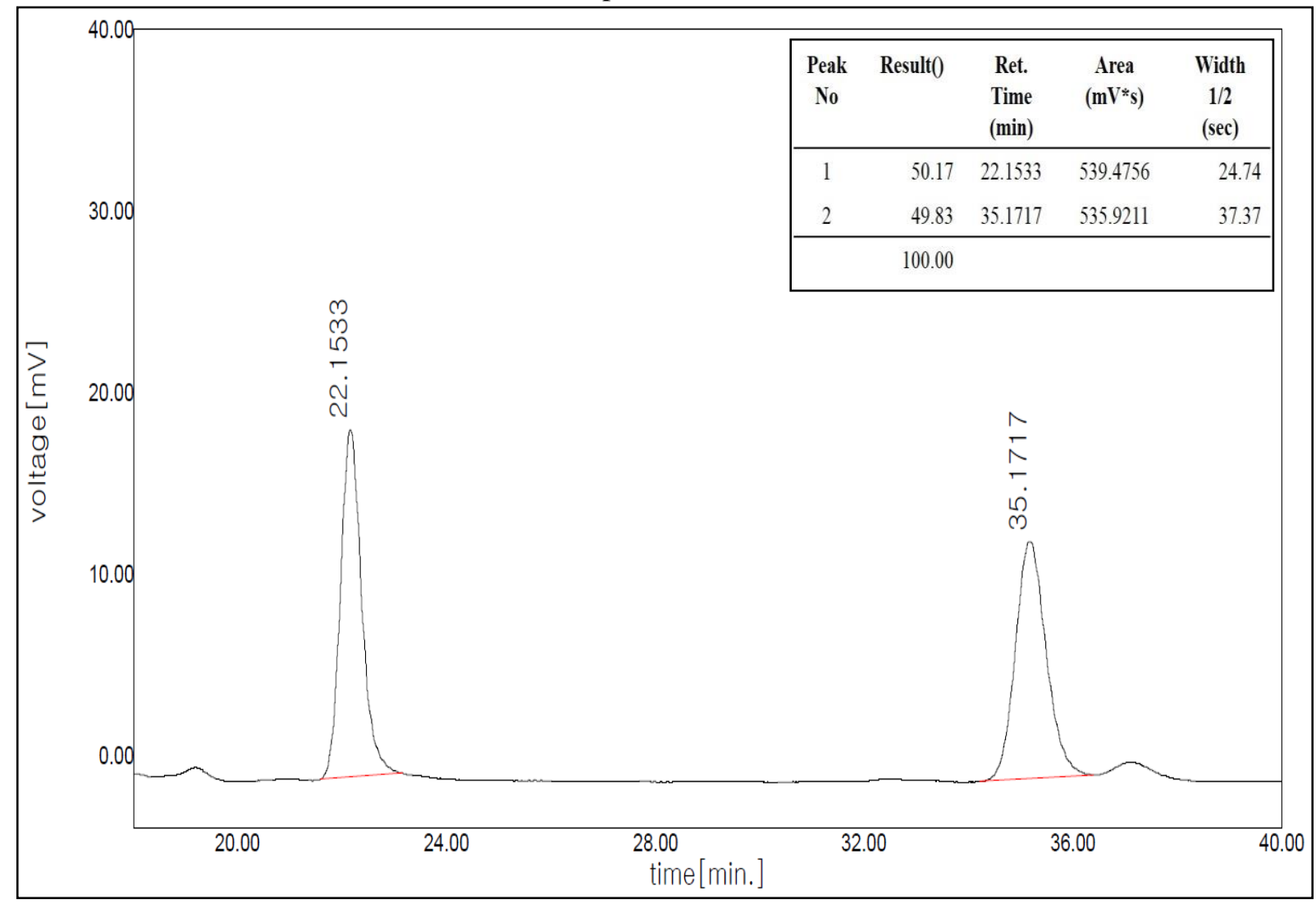

HPLC spectrum of $r a c-3 \mathbf{g}$ 


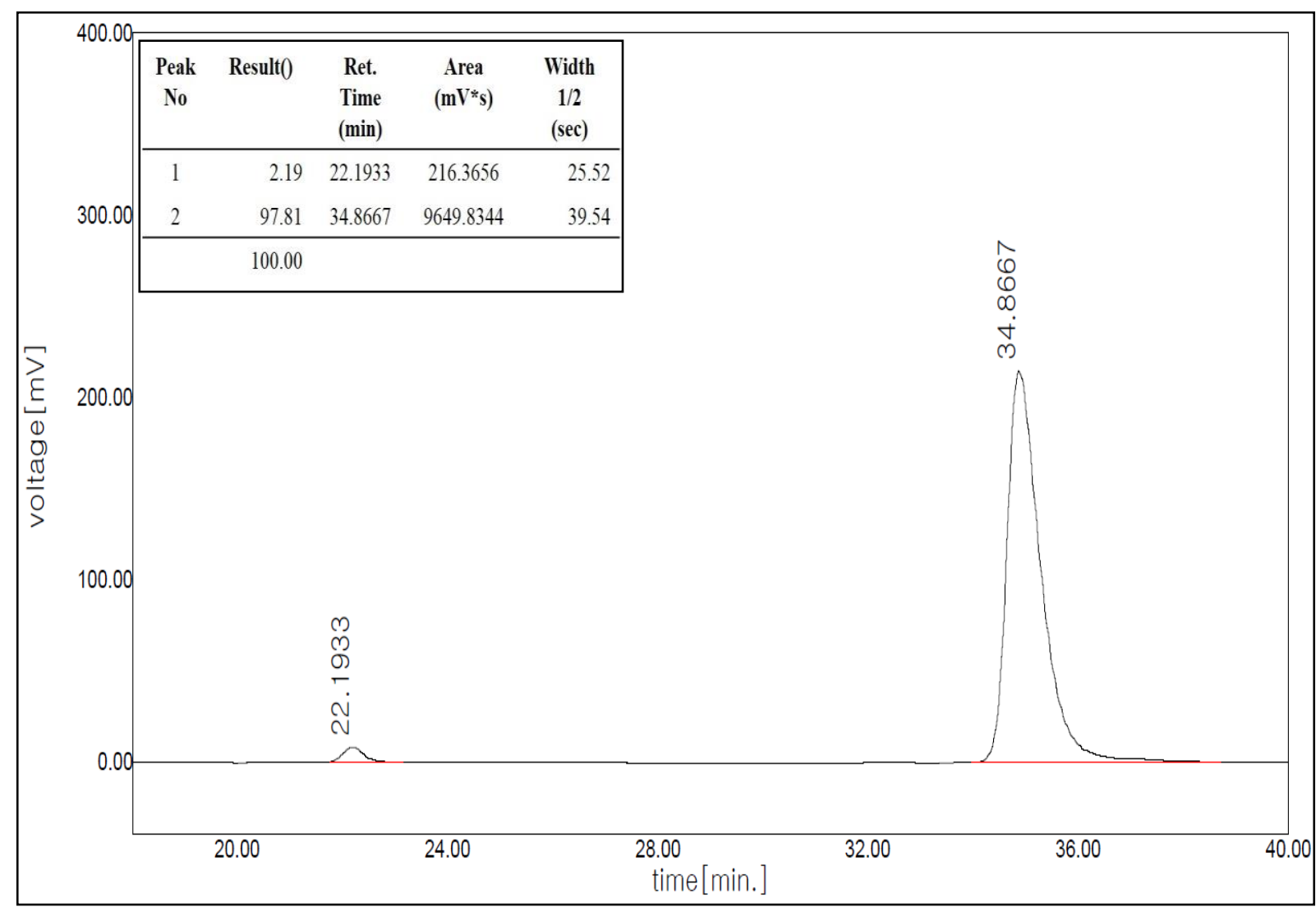

HPLC spectrum of $\mathbf{3 g}$

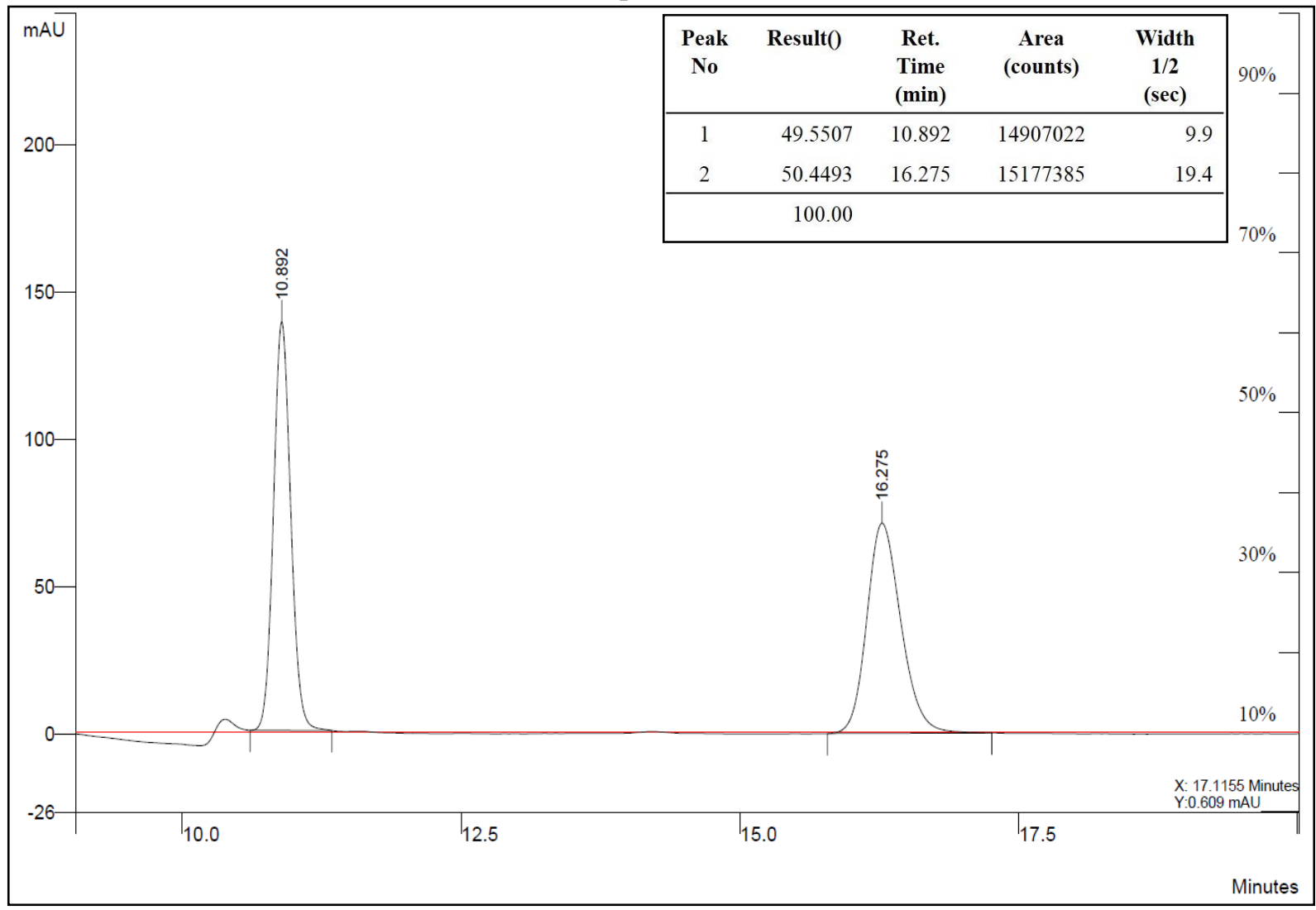

HPLC spectrum of $r a c-3 \mathbf{h}$ 


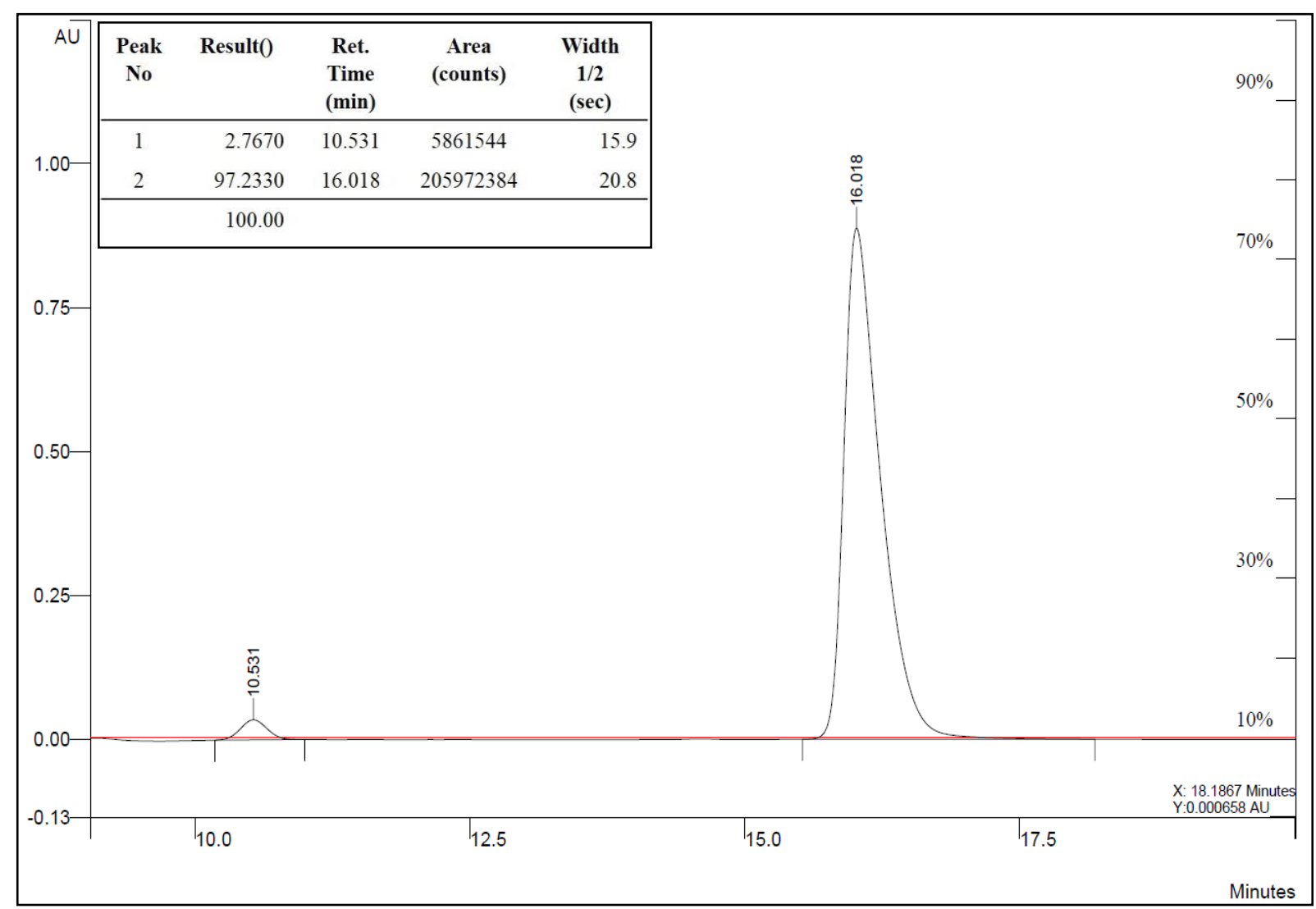

HPLC spectrum of $\mathbf{3 h}$

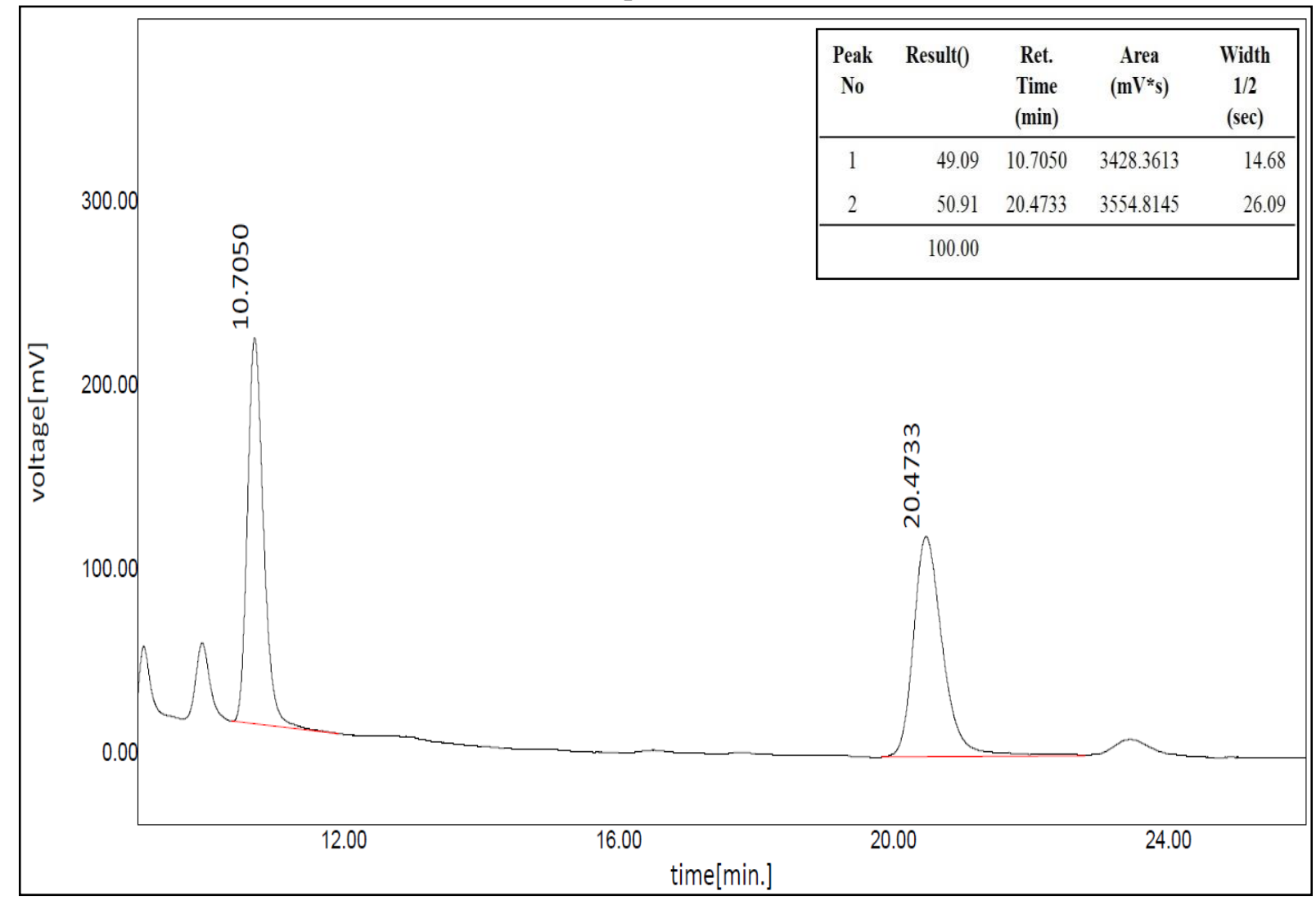

HPLC spectrum of $r a c-3 i$ 


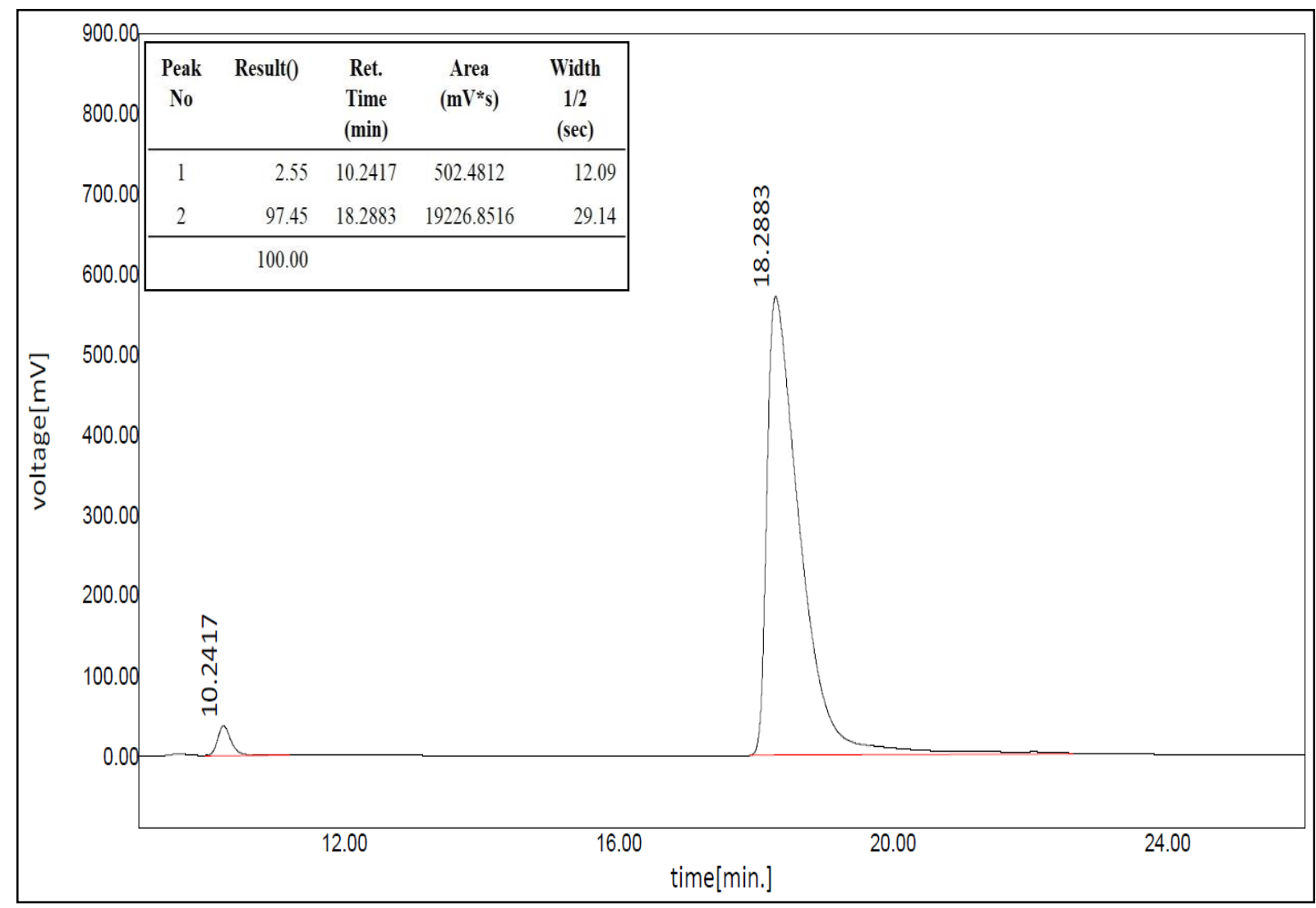

HPLC spectrum of $\mathbf{3 i}$

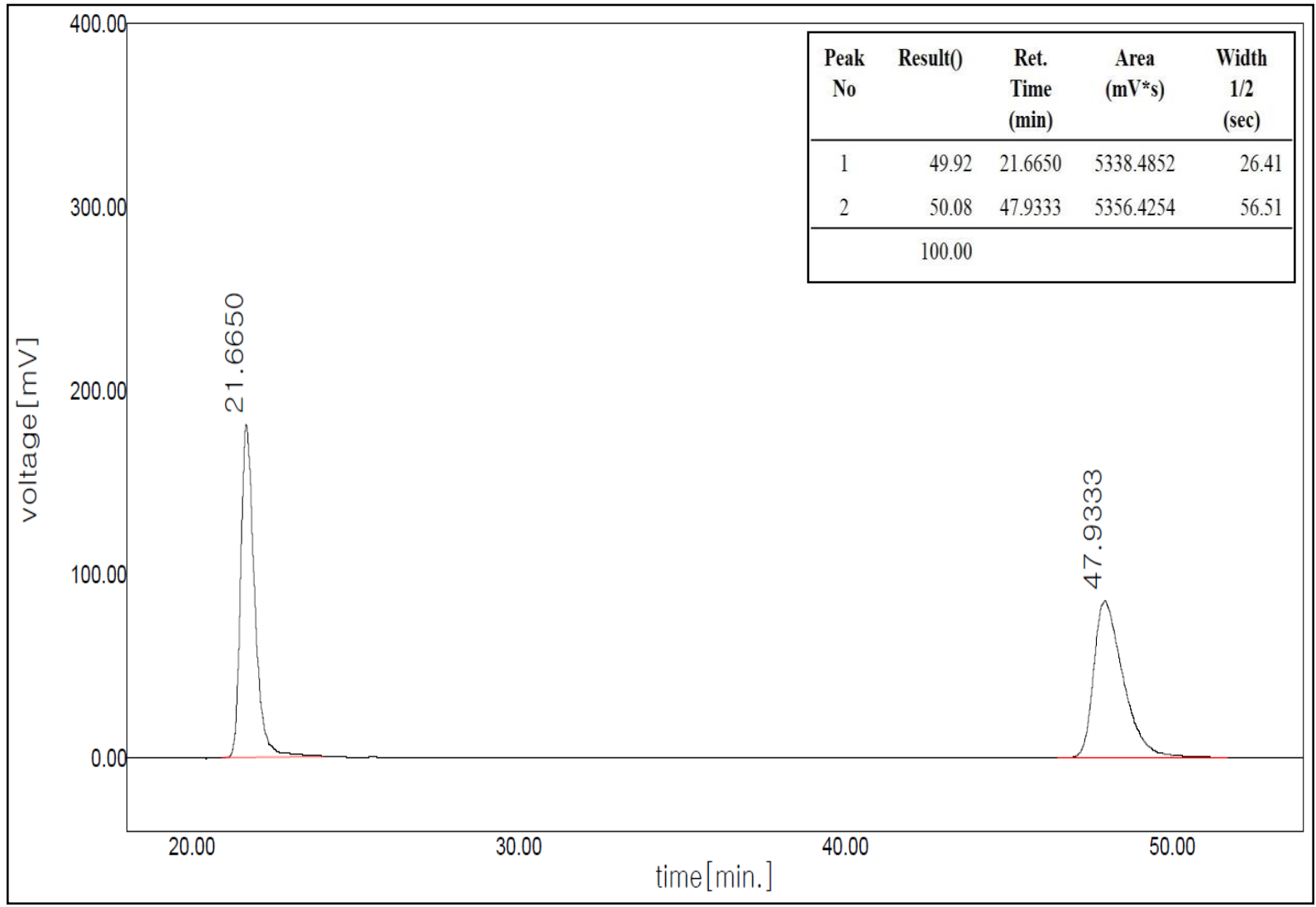

HPLC spectrum of $r a c-3 \mathbf{j}$ 


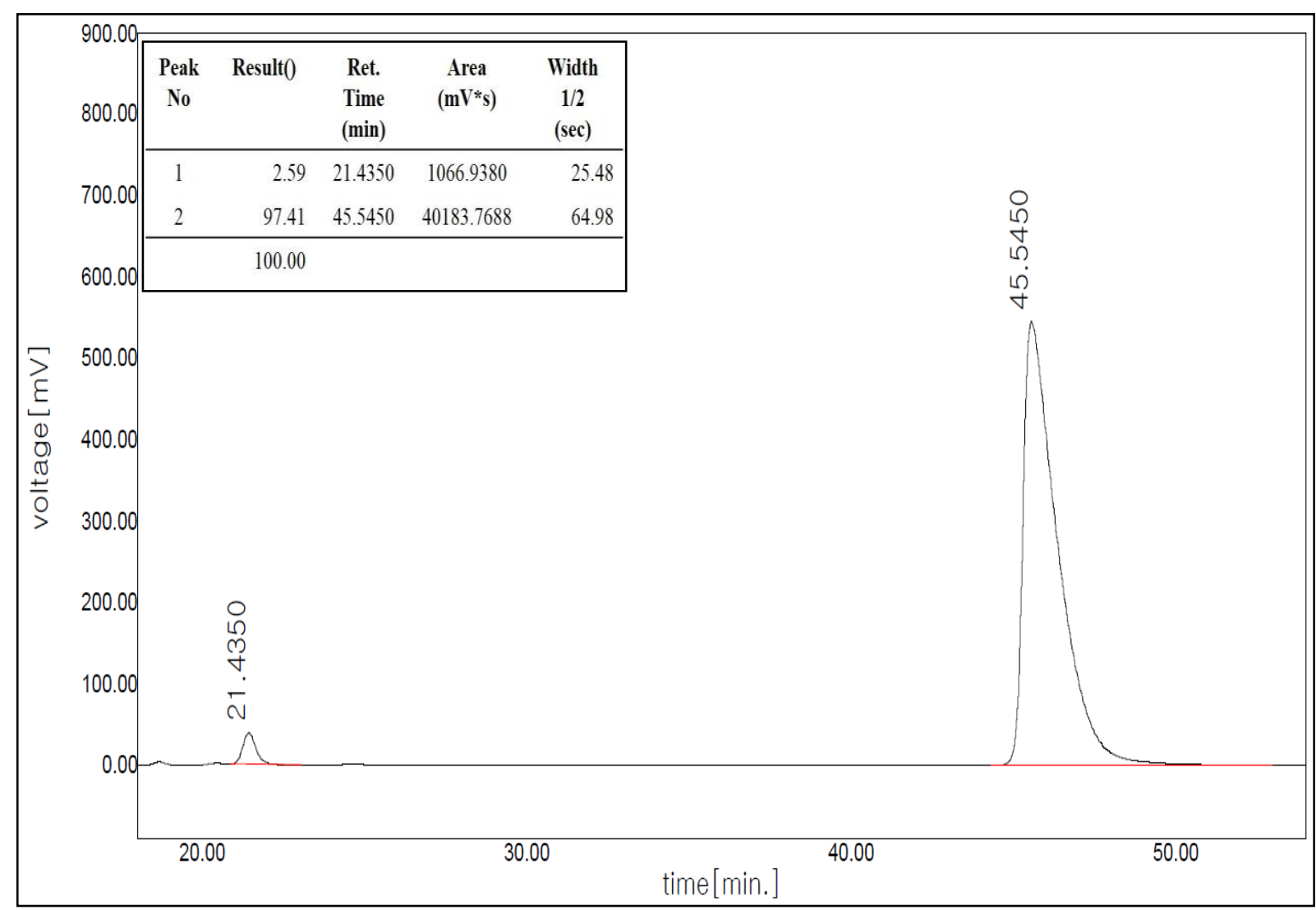

HPLC spectrum of $\mathbf{3 j}$

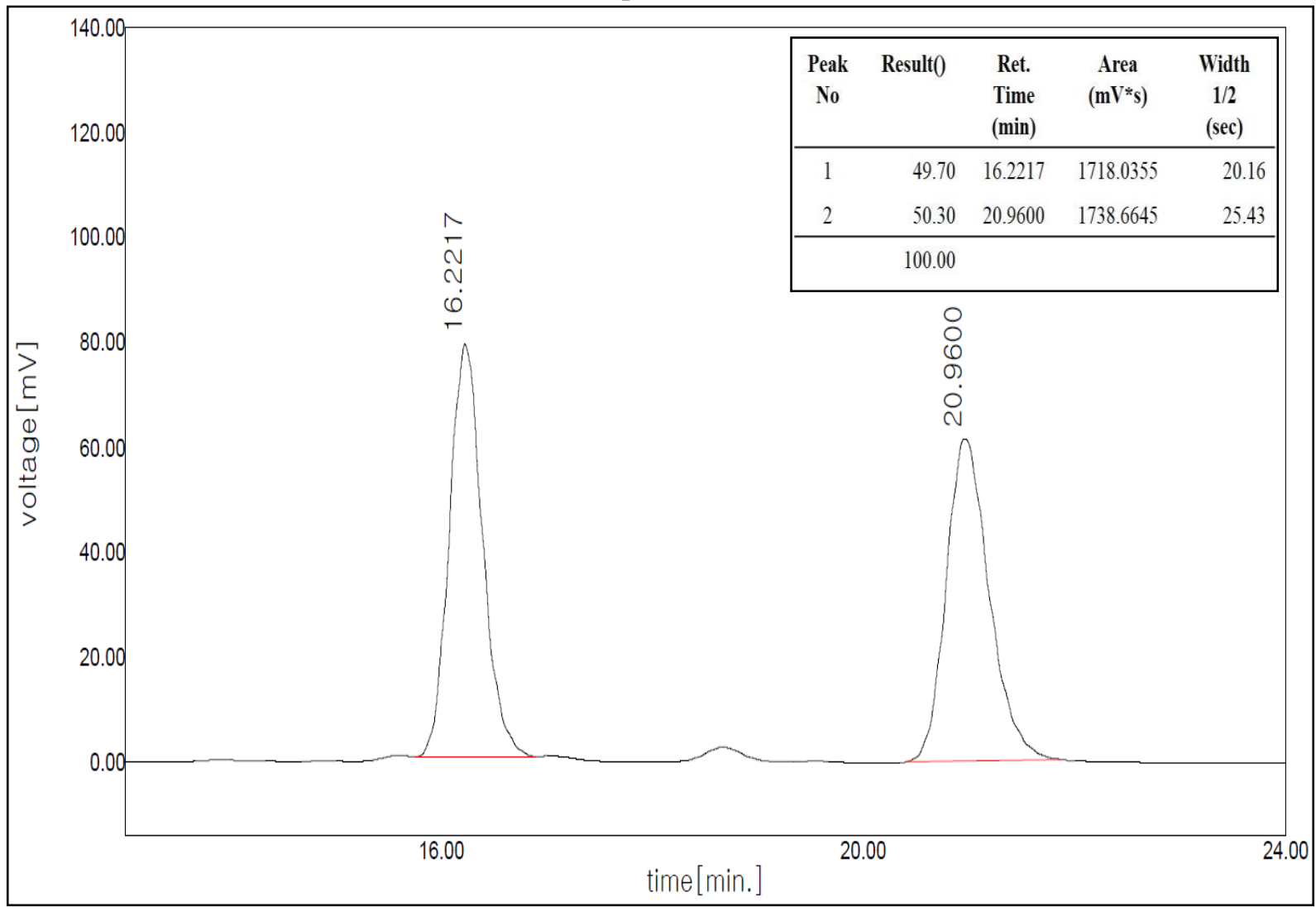

HPLC spectrum of $r a c-3 \mathbf{k}$ 


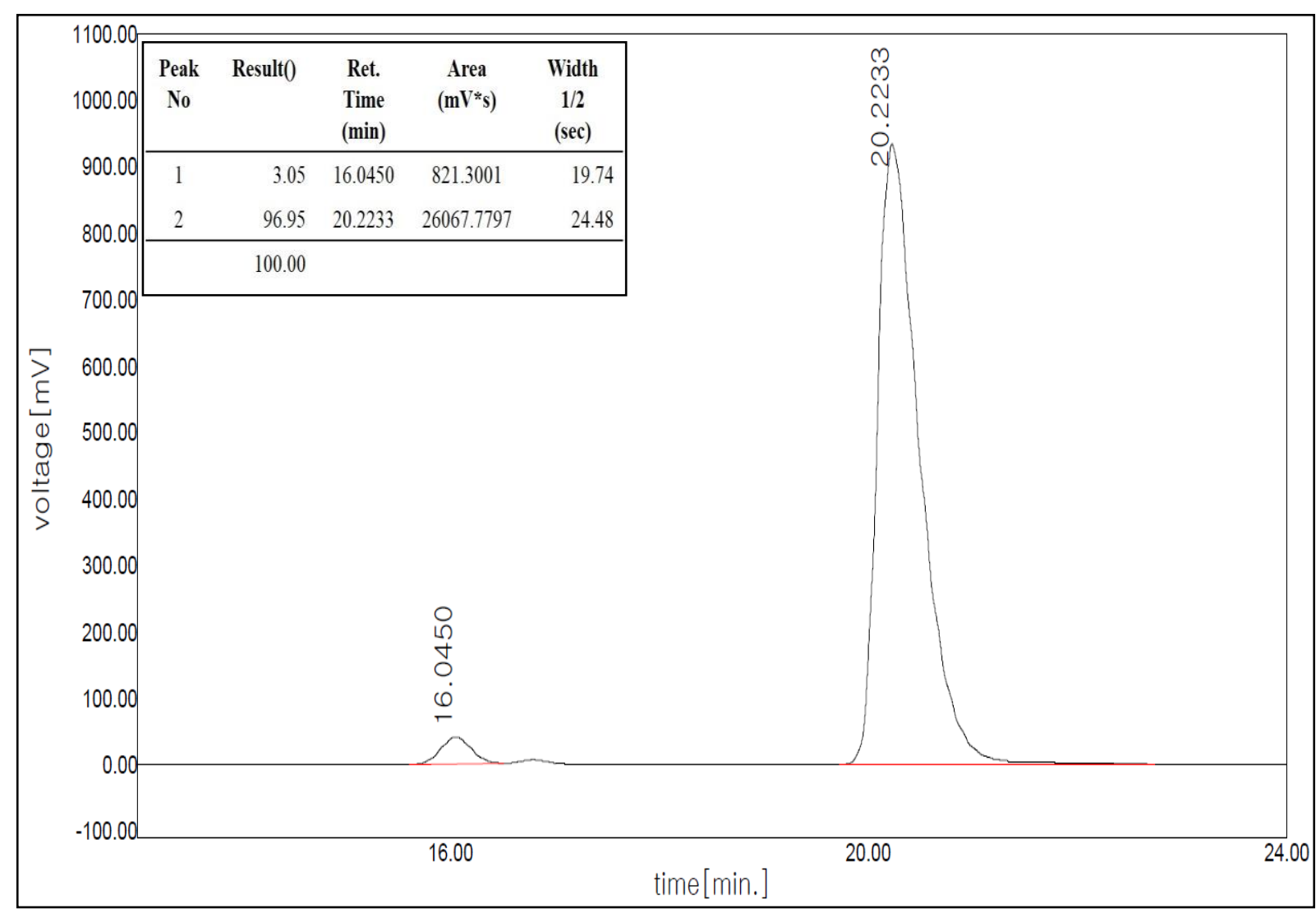

HPLC spectrum of $\mathbf{3 k}$

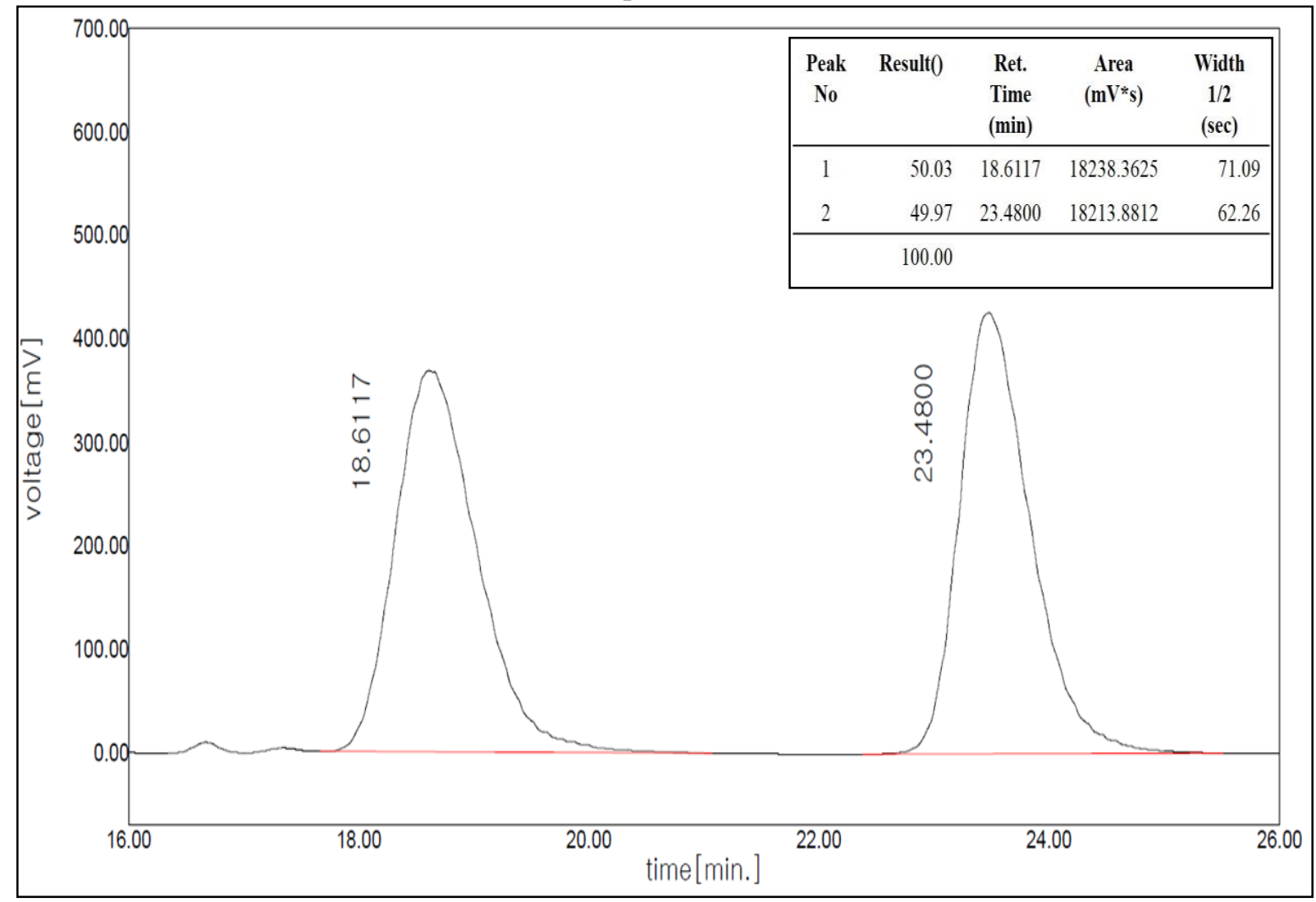

HPLC spectrum of rac-3I 


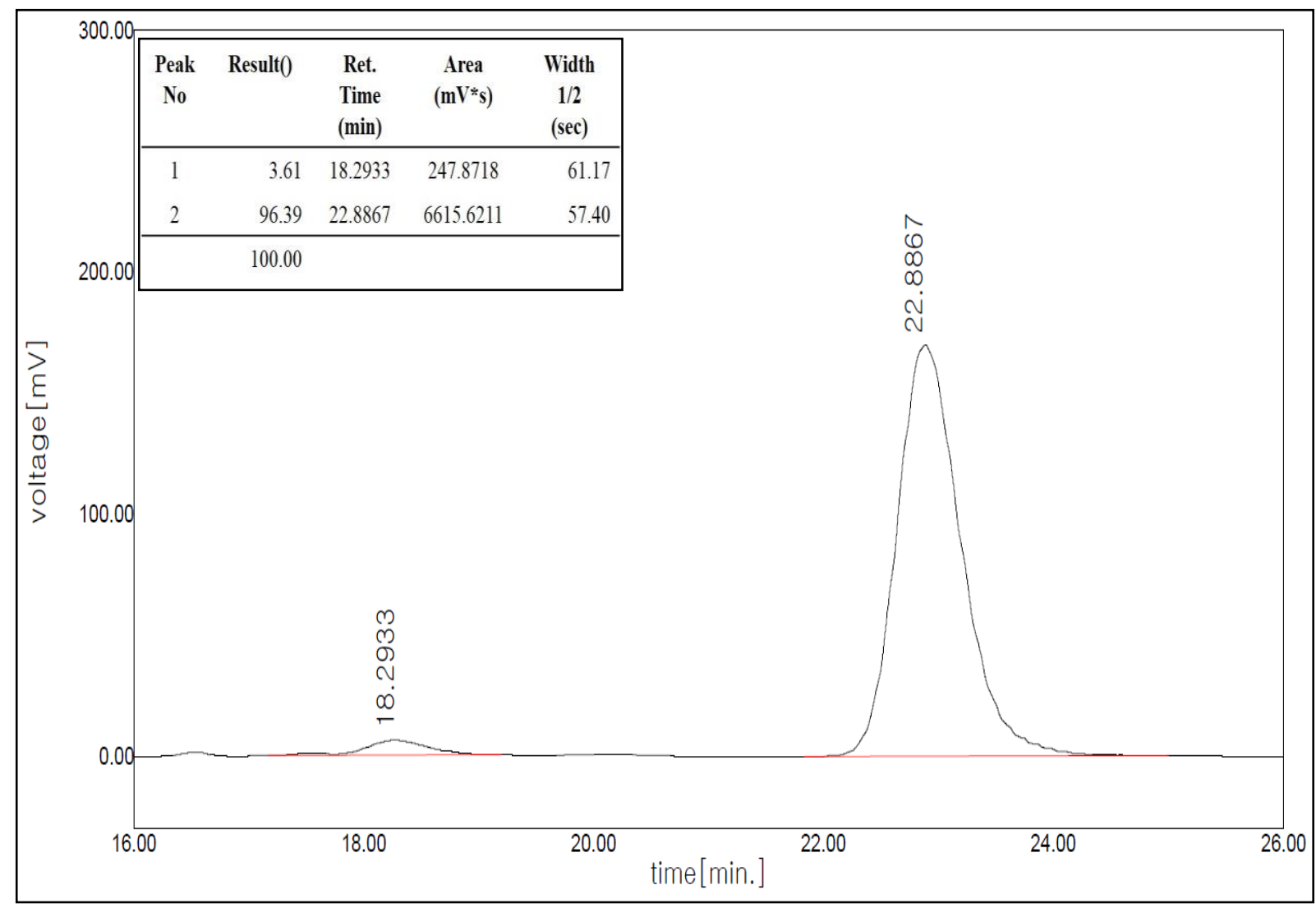

HPLC spectrum of $\mathbf{3 l}$

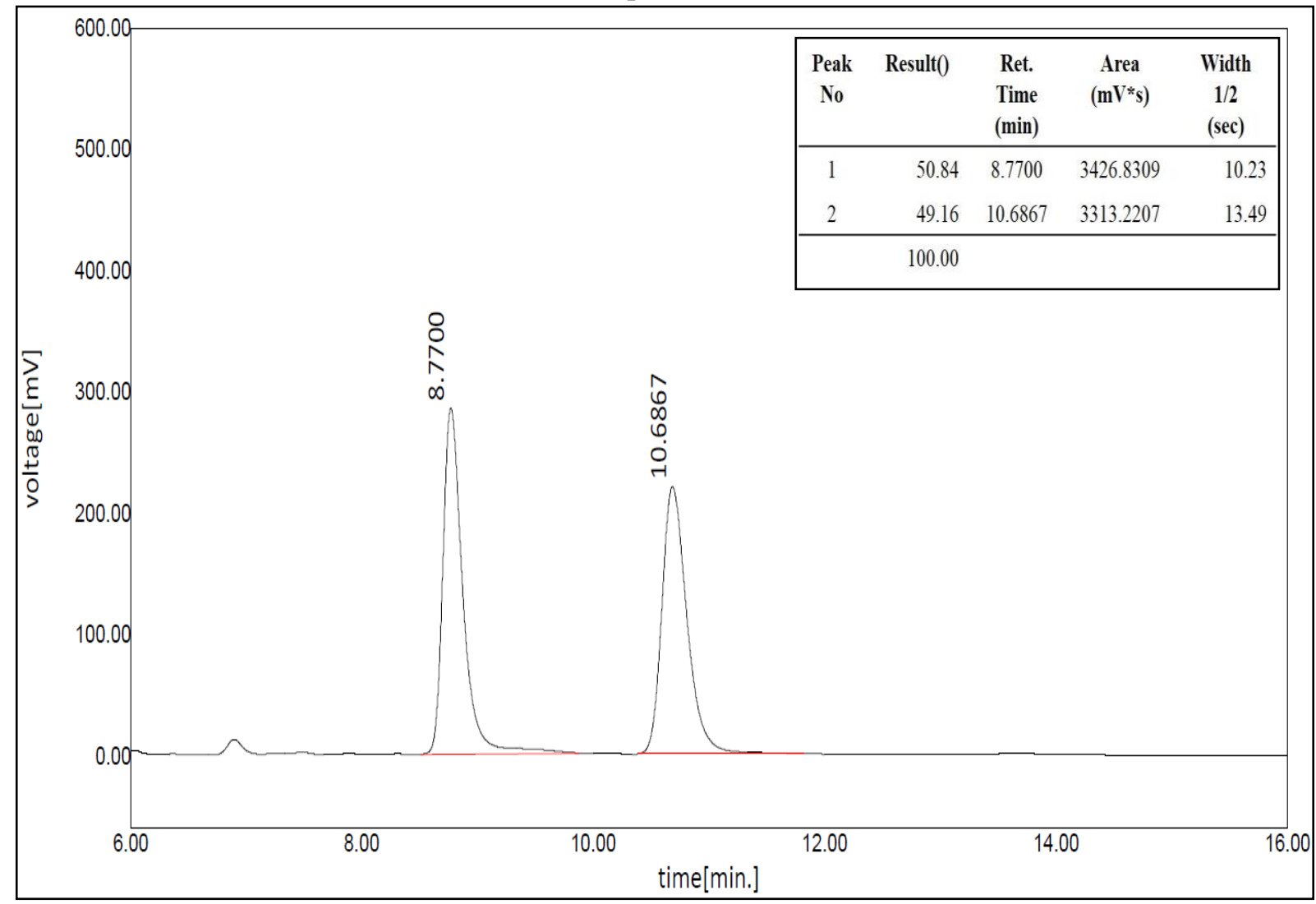

HPLC spectrum of $\mathrm{rac}-\mathbf{3 m}$ 


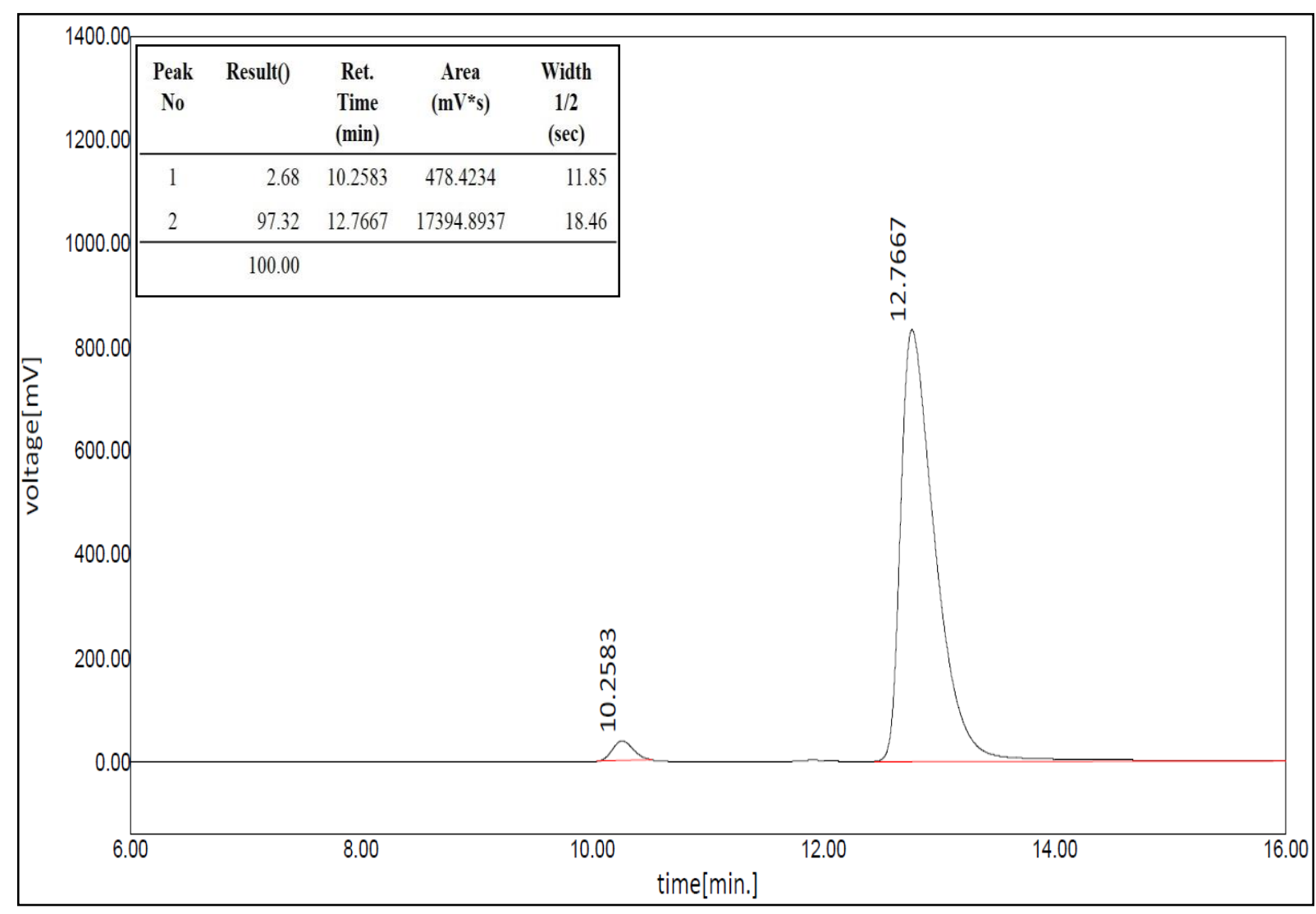

HPLC spectrum of $\mathbf{3 m}$

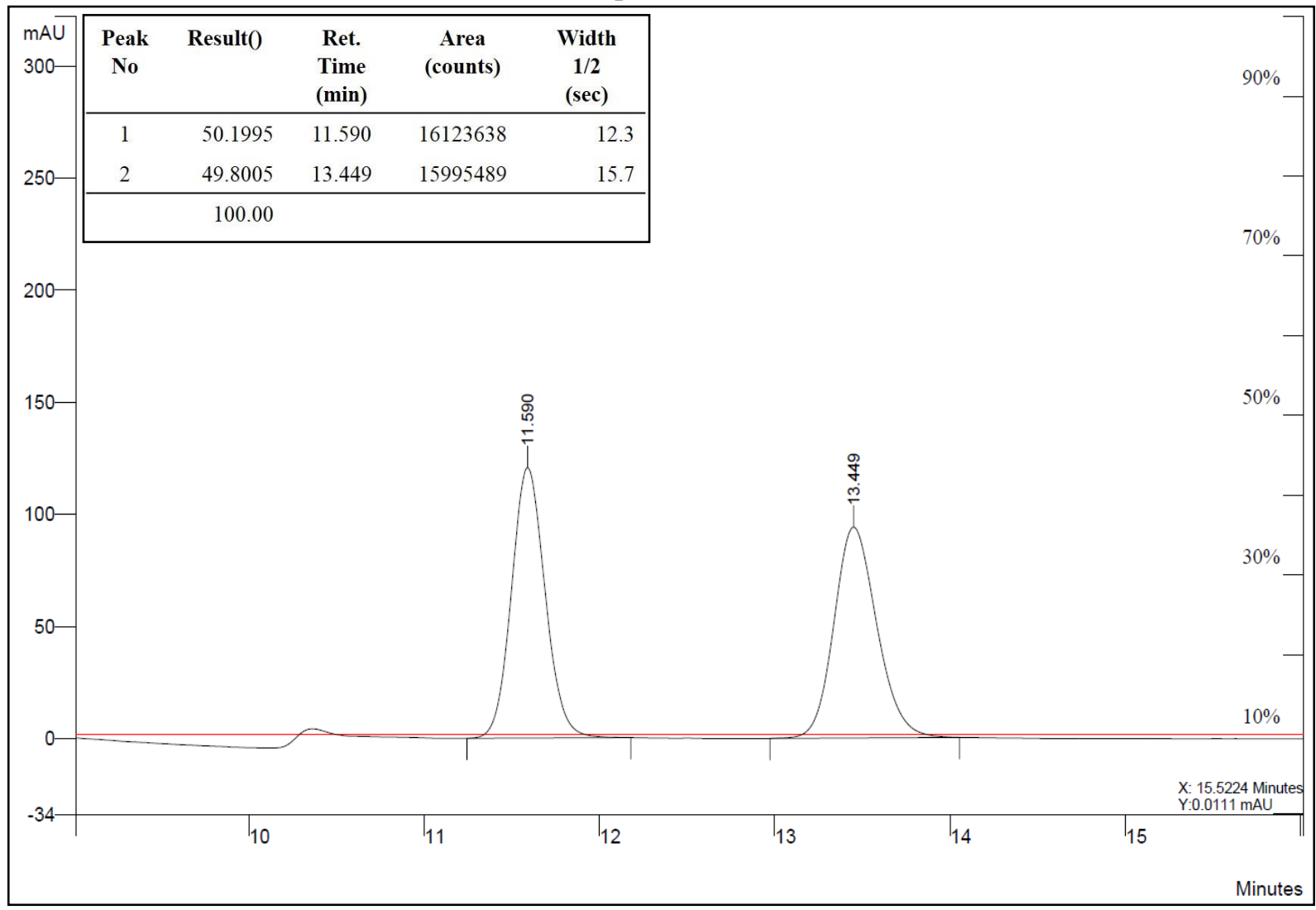

HPLC spectrum of $r a c-3 \mathbf{n}$ 


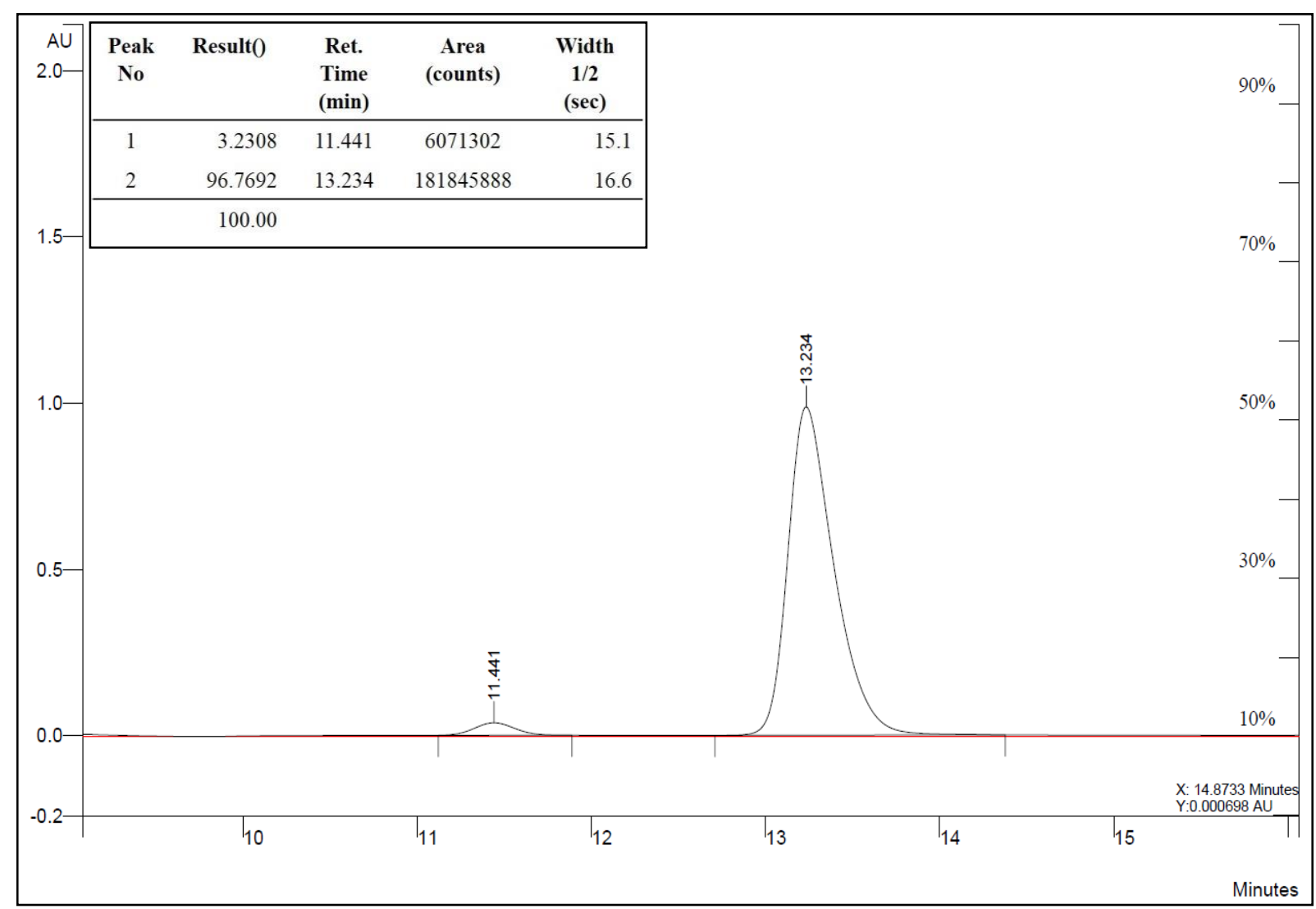

HPLC spectrum of $\mathbf{3 n}$

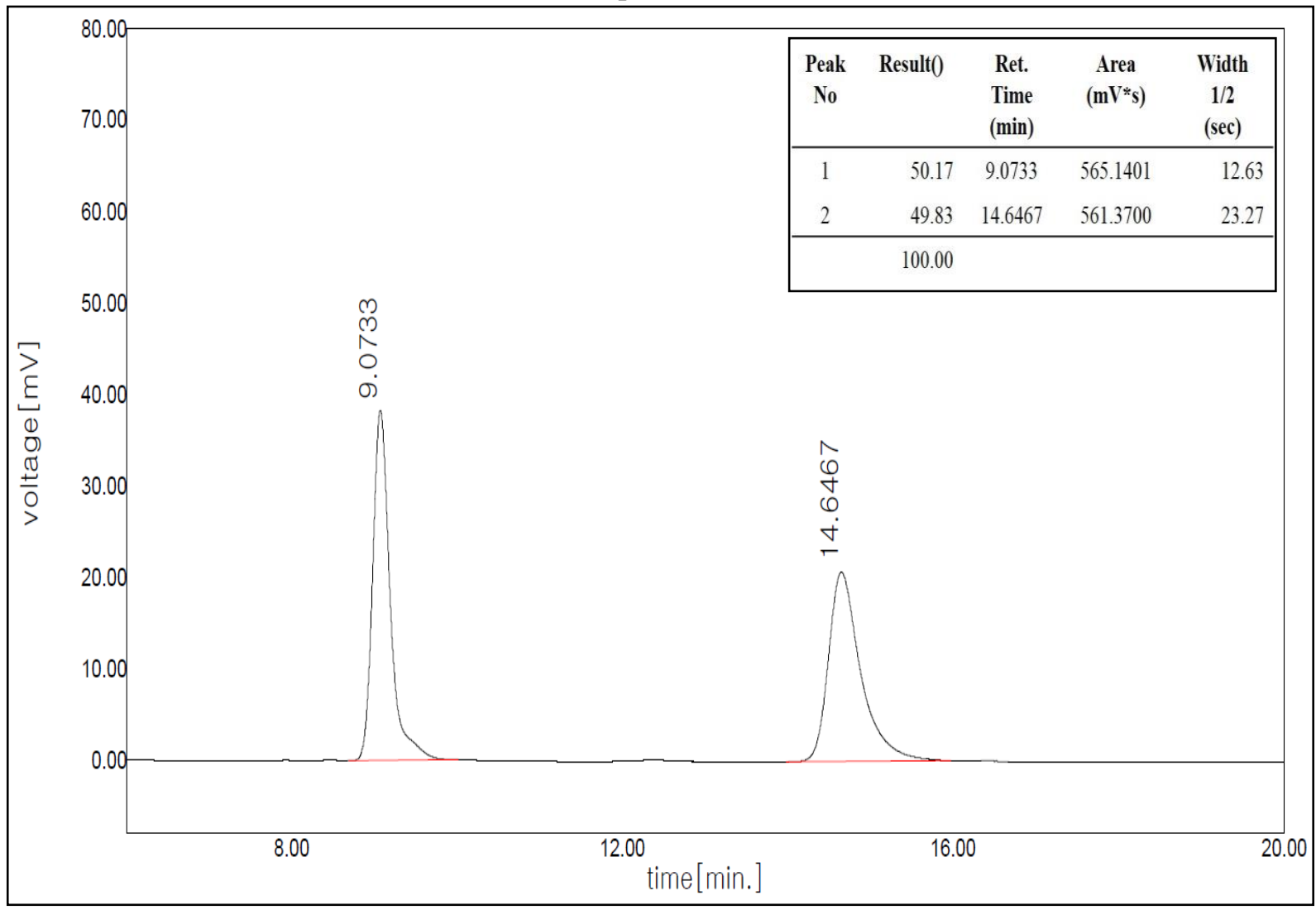

HPLC spectrum of $r a c-30$ 


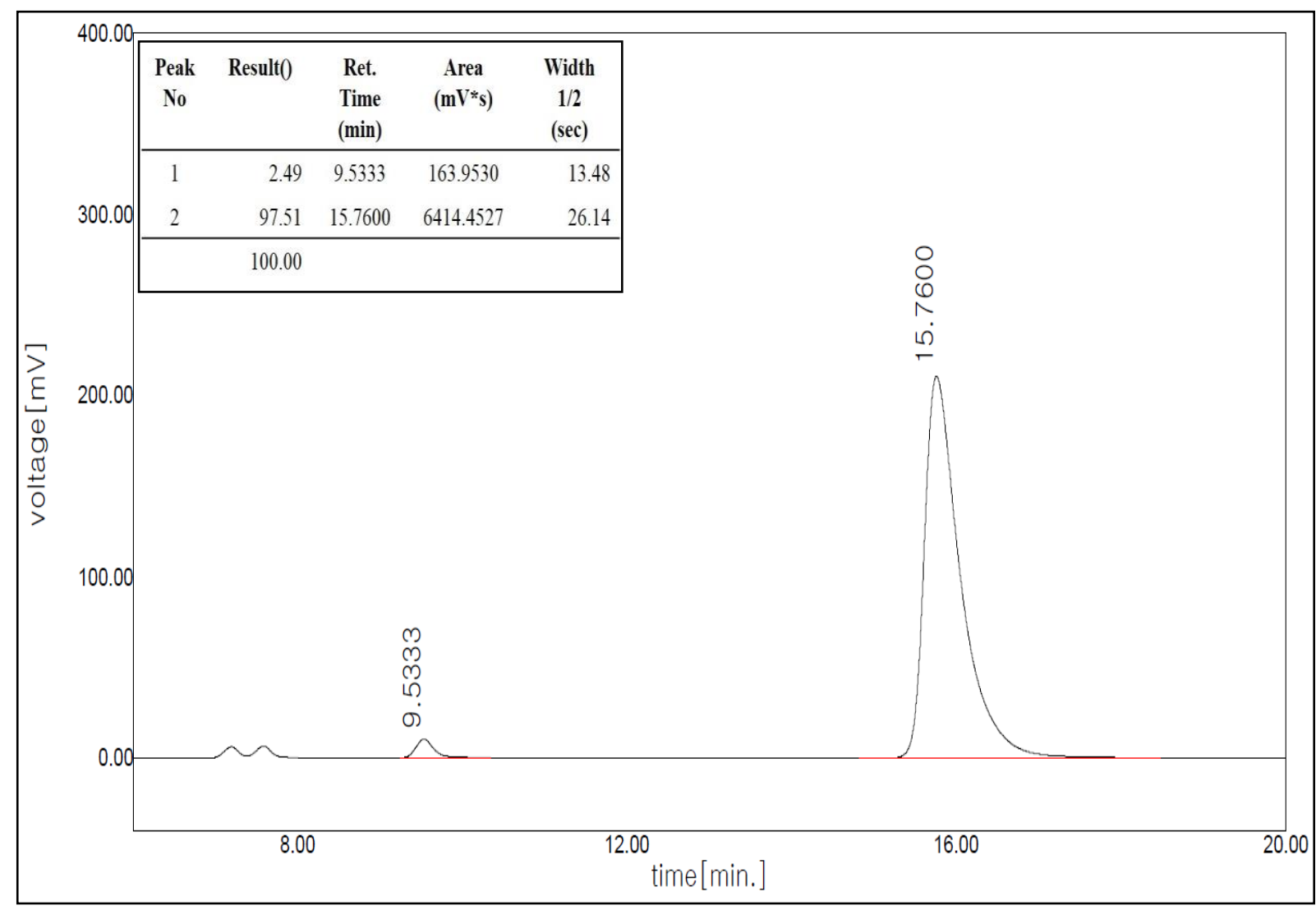

HPLC spectrum of $\mathbf{3 o}$

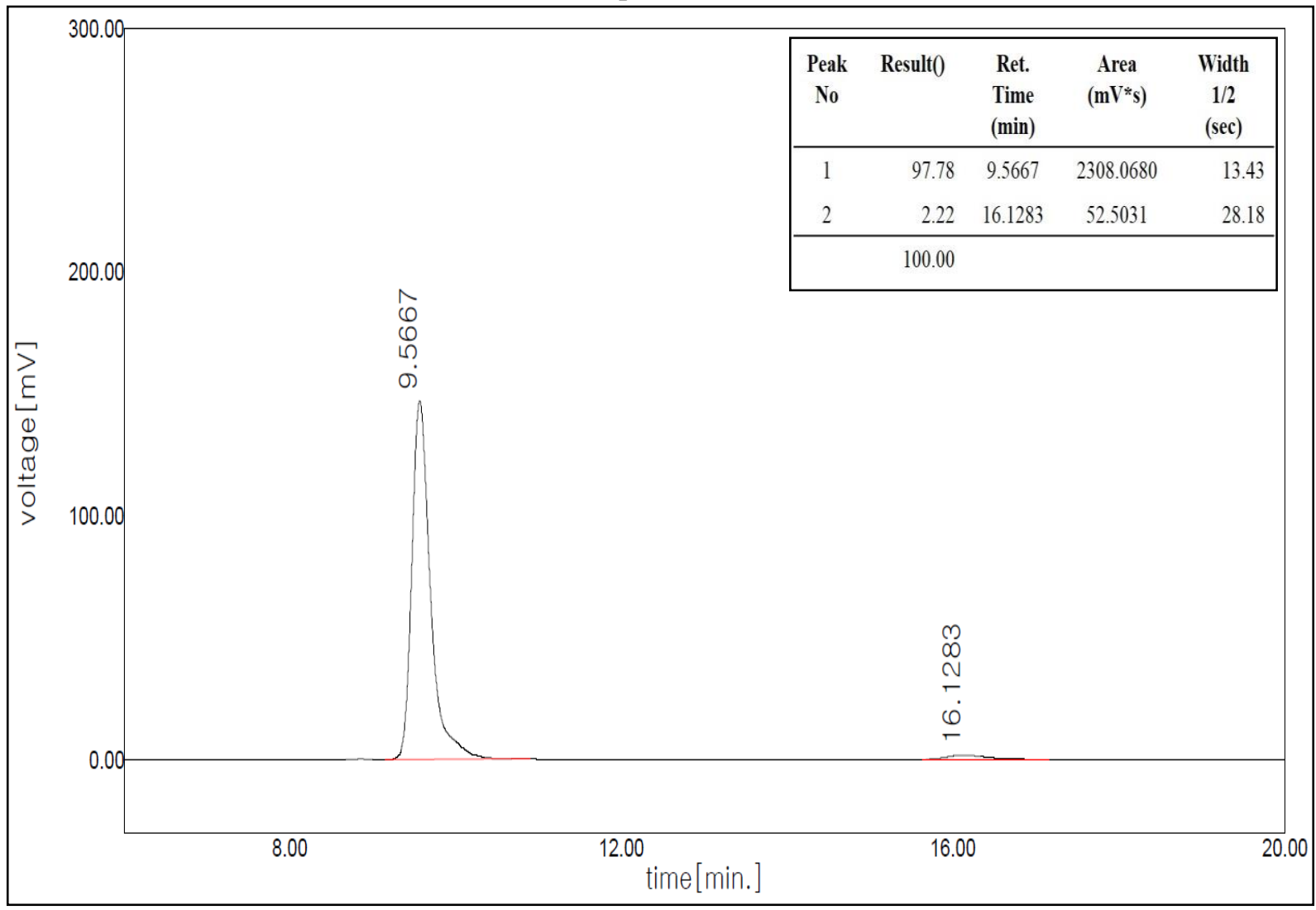

HPLC spectrum of ent-3o 


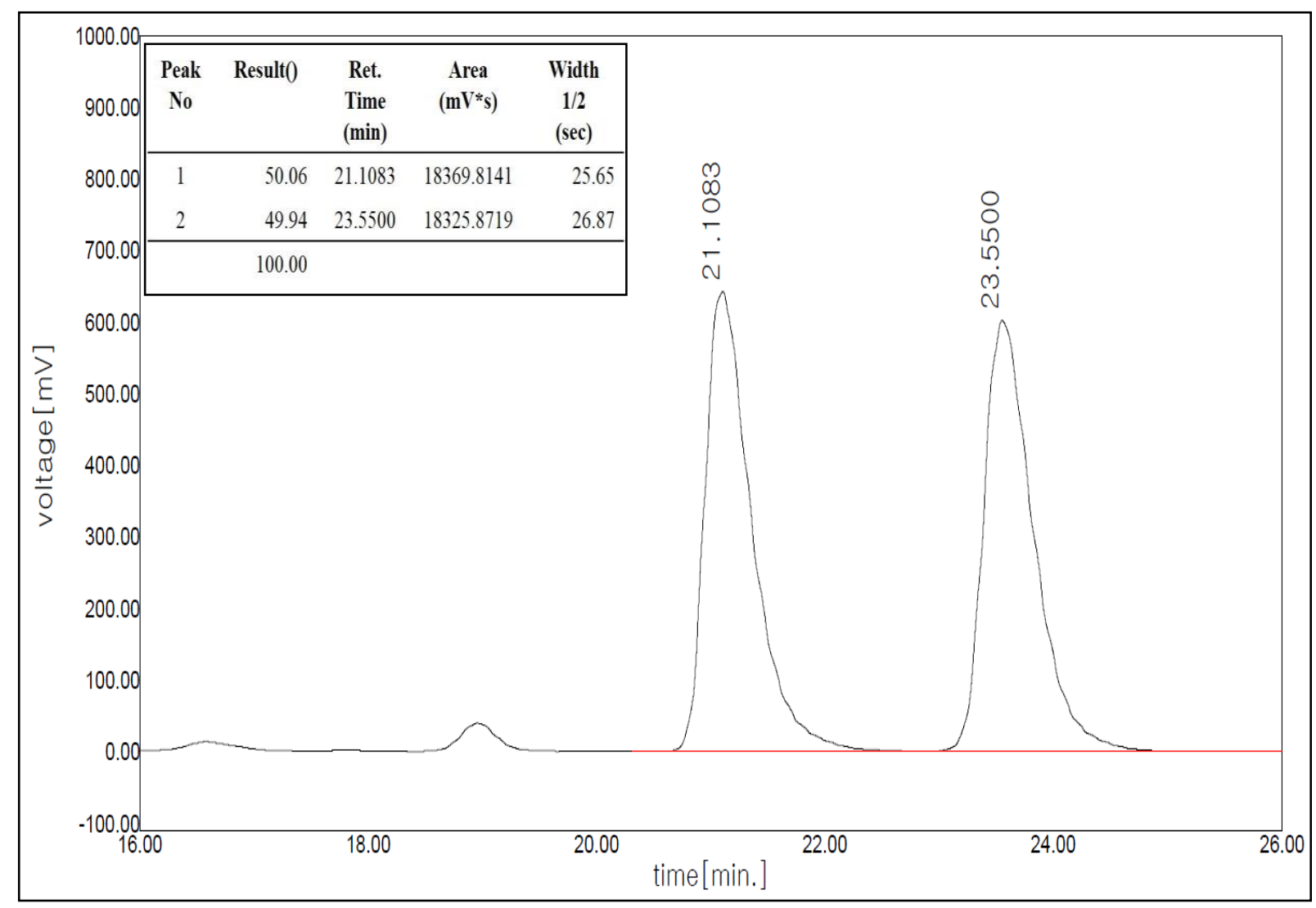

HPLC spectrum of $r a c-\mathbf{3 p}$

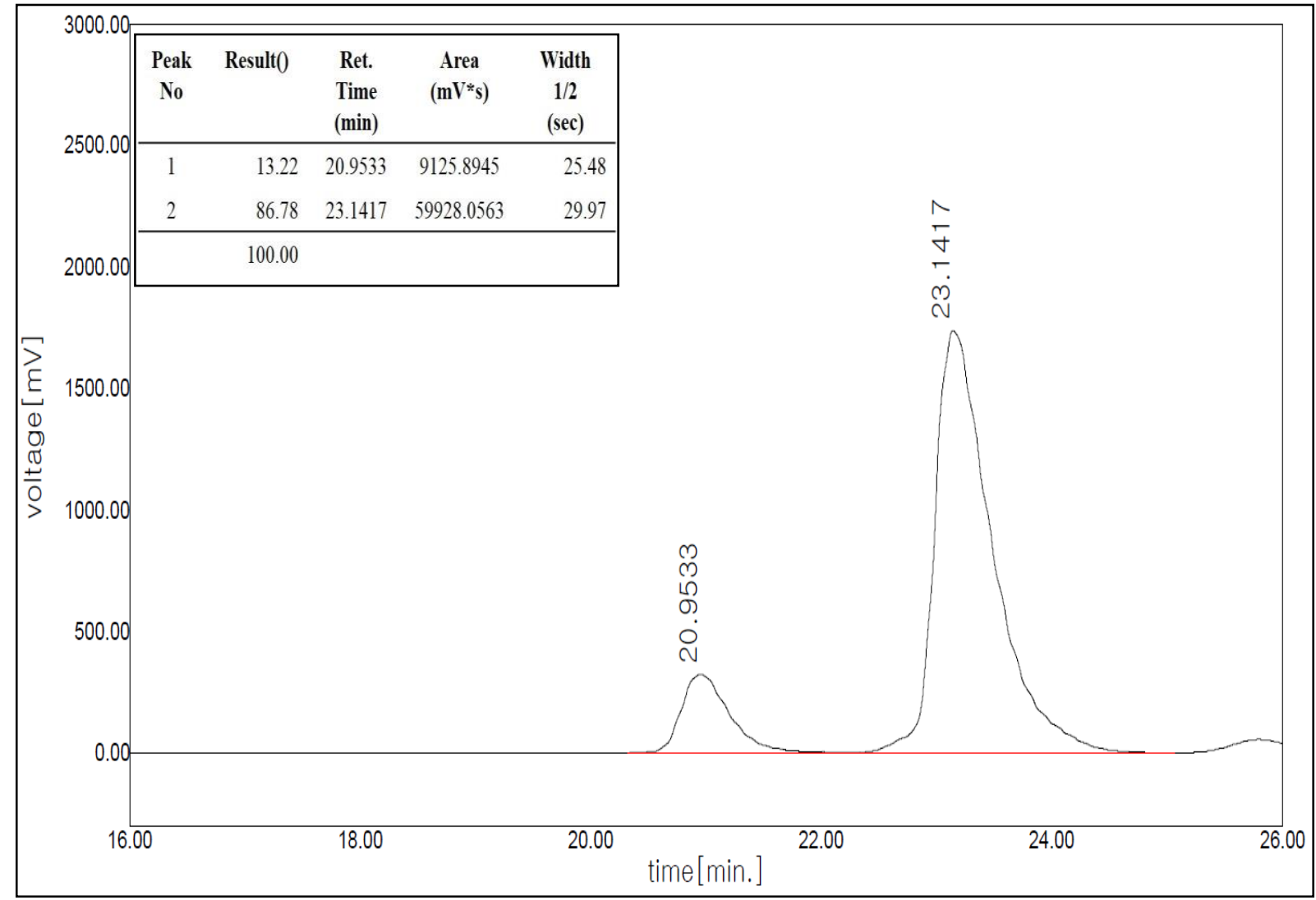

HPLC spectrum of $\mathbf{3 p}$ 


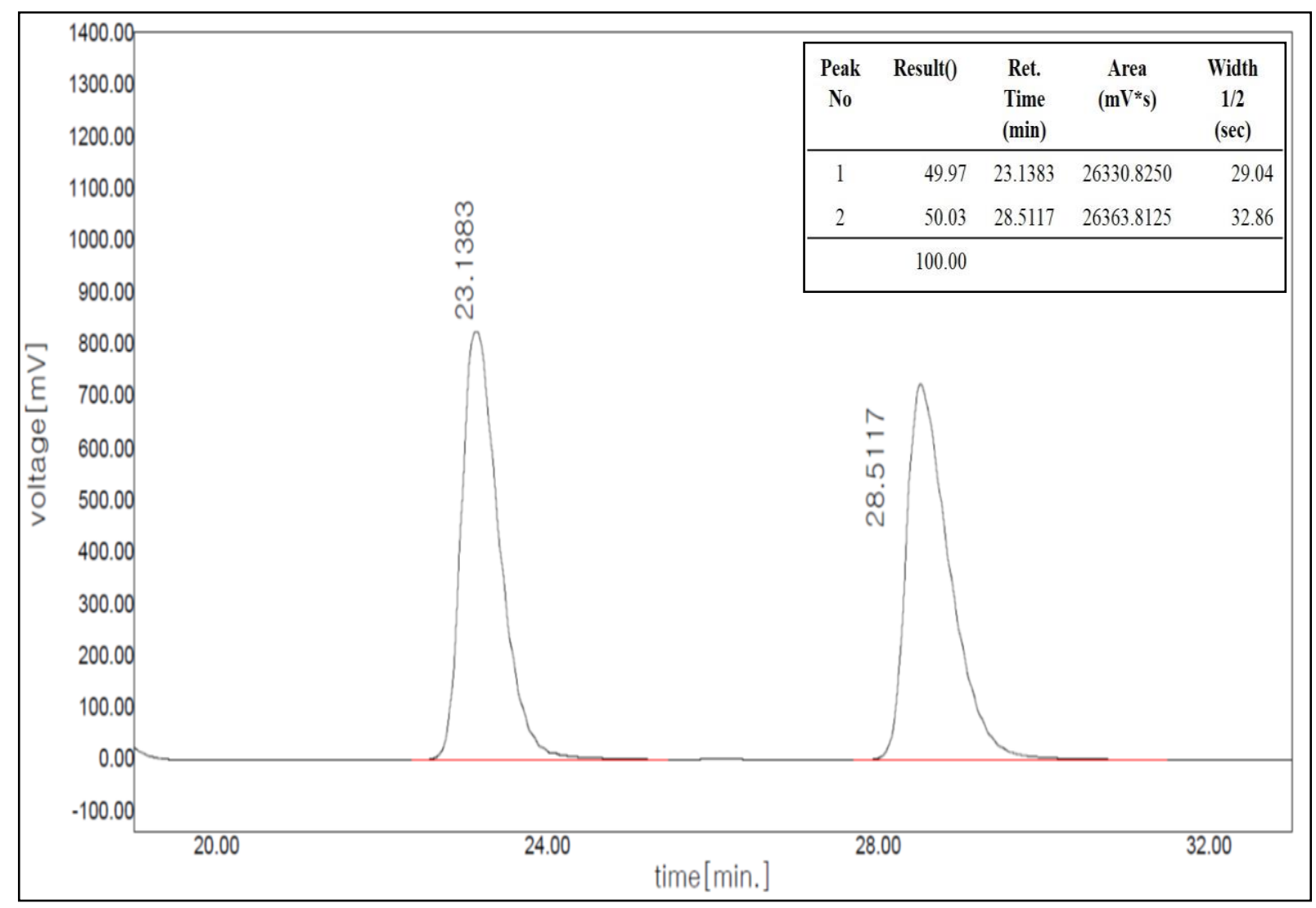

HPLC spectrum of $r a c-\mathbf{3 q}$

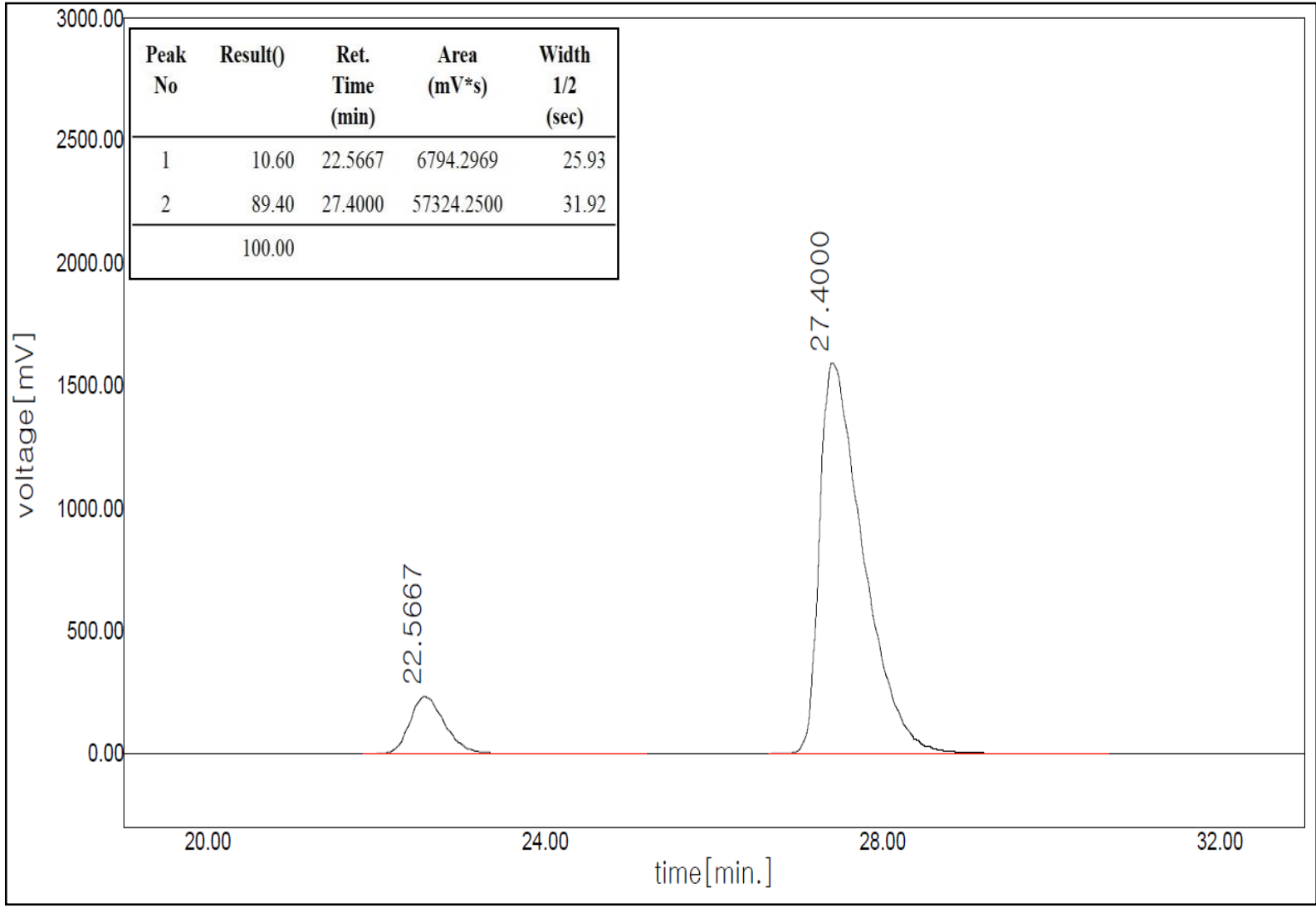

HPLC spectrum of $\mathbf{3 q}$ 


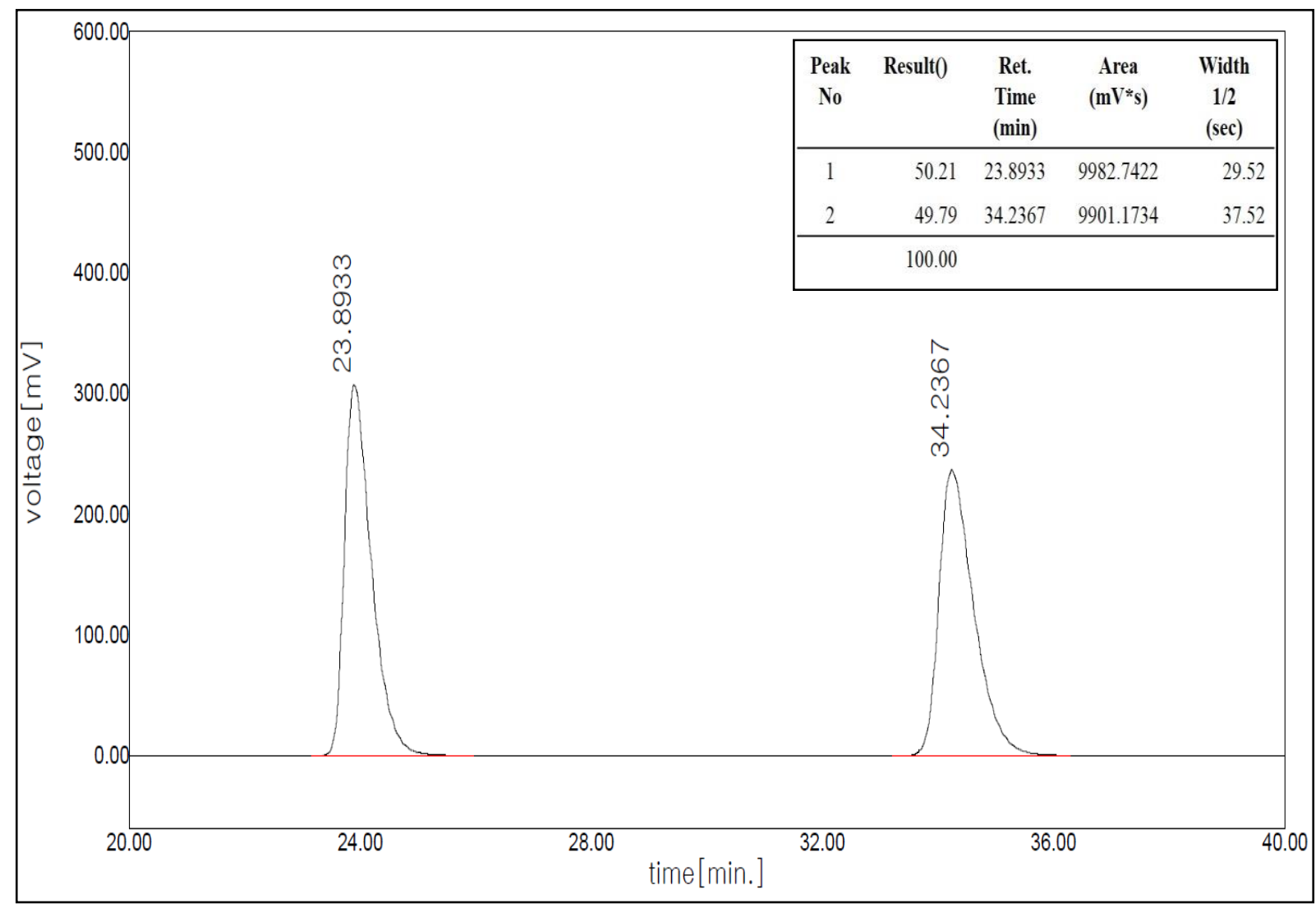

HPLC spectrum of $r a c-3 \mathbf{r}$

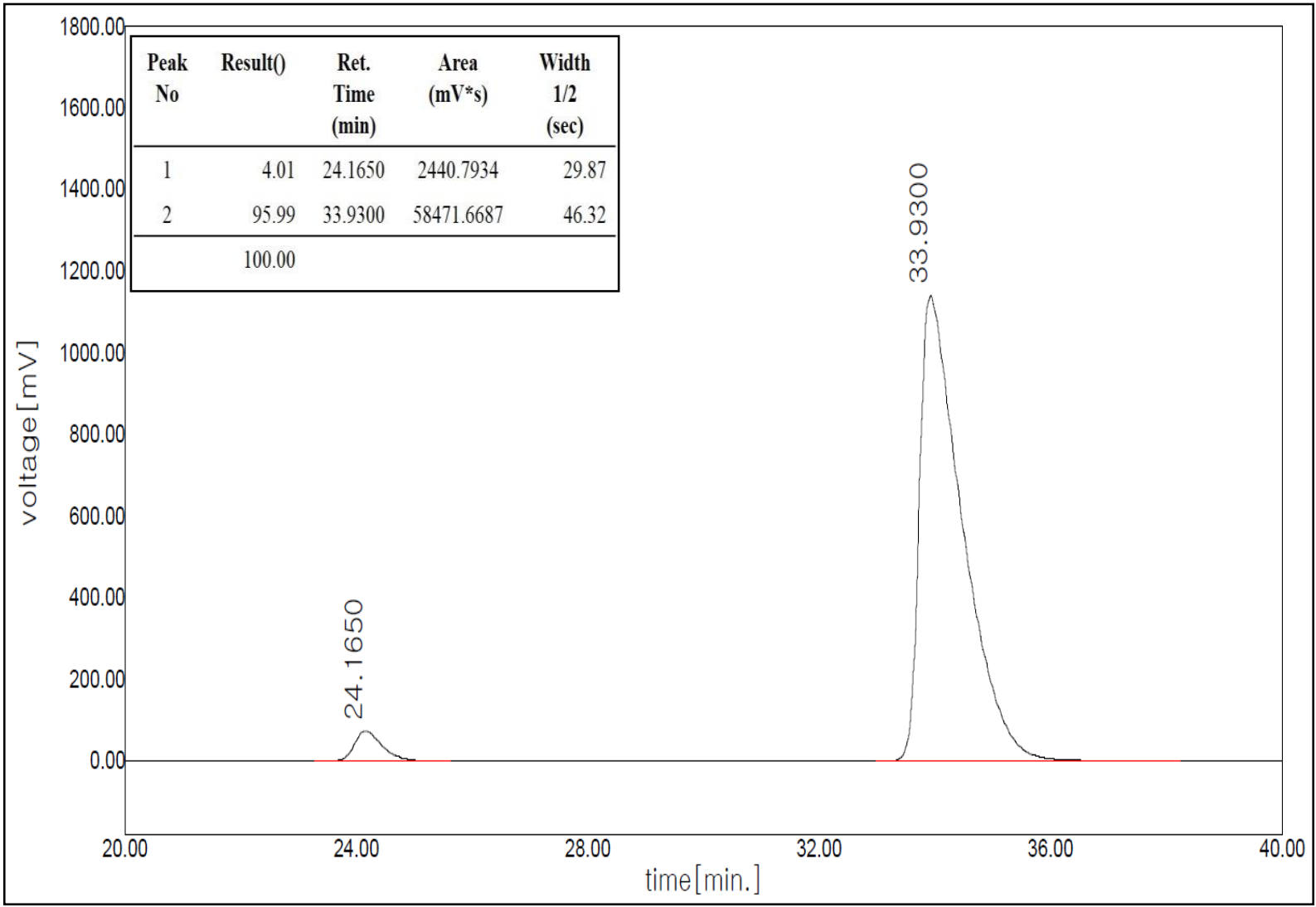

HPLC spectrum of $\mathbf{3 r}$ 


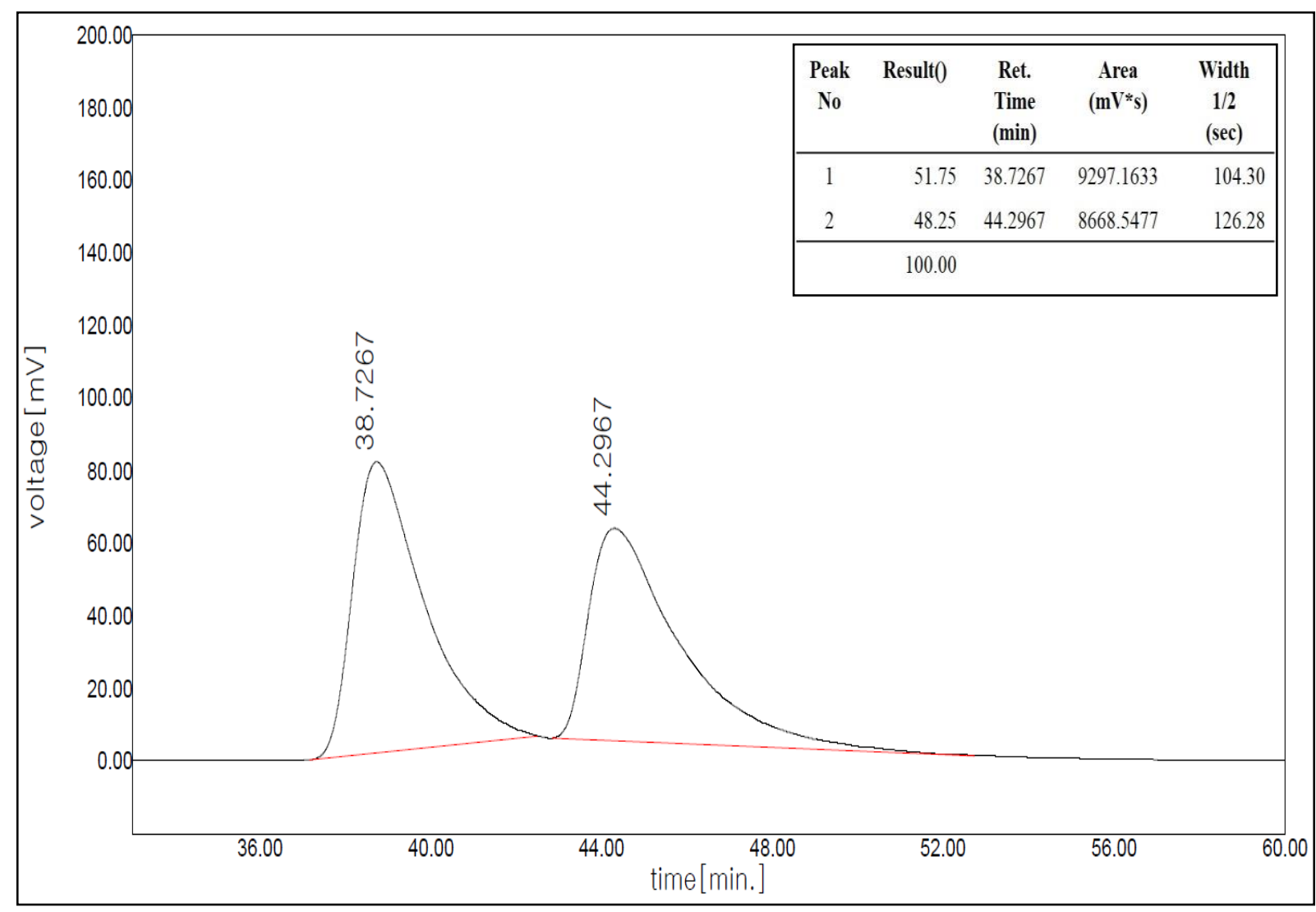

HPLC spectrum of $\mathrm{rac}-\mathbf{3 s}$

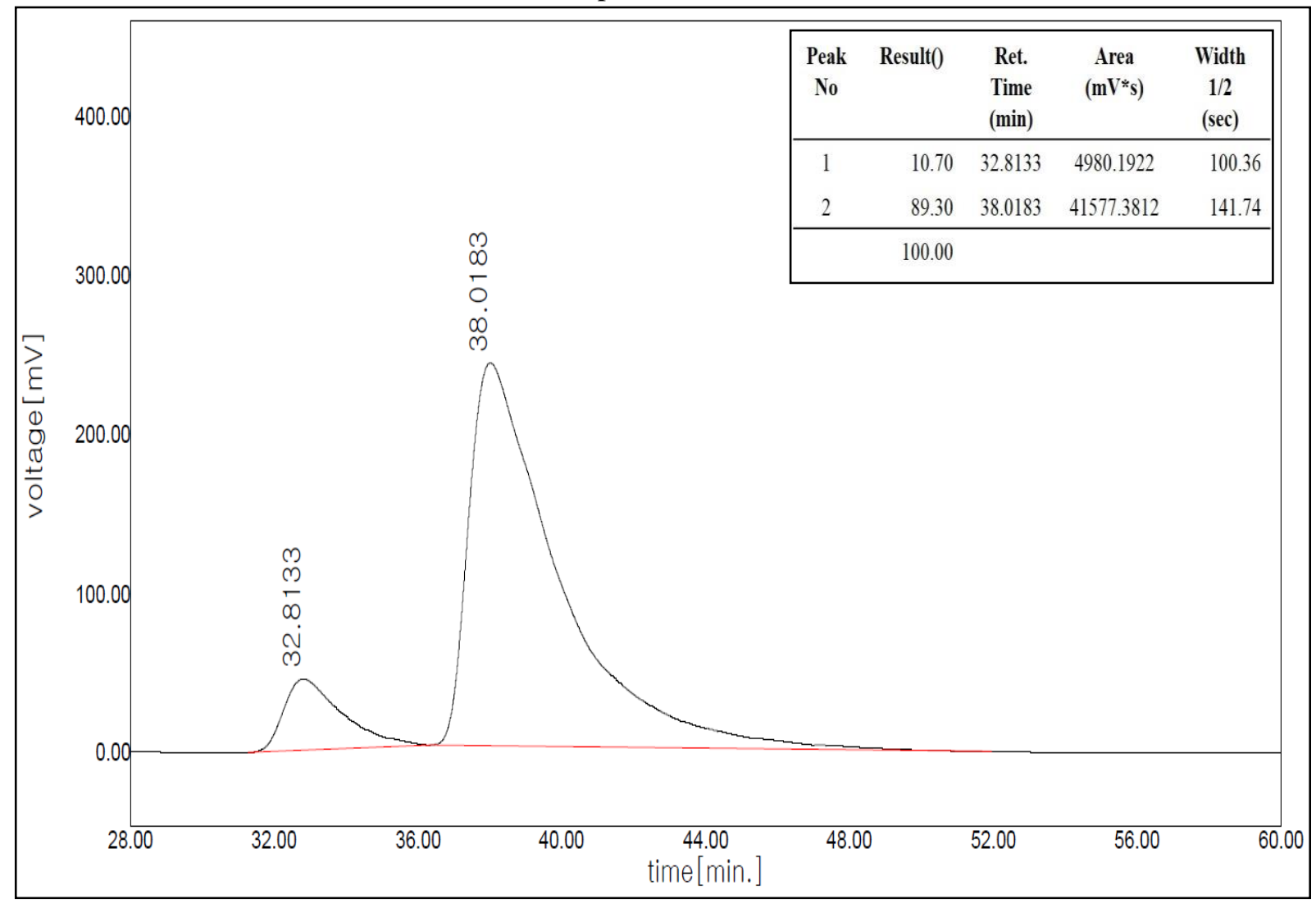

HPLC spectrum of $3 \mathbf{s}$ 


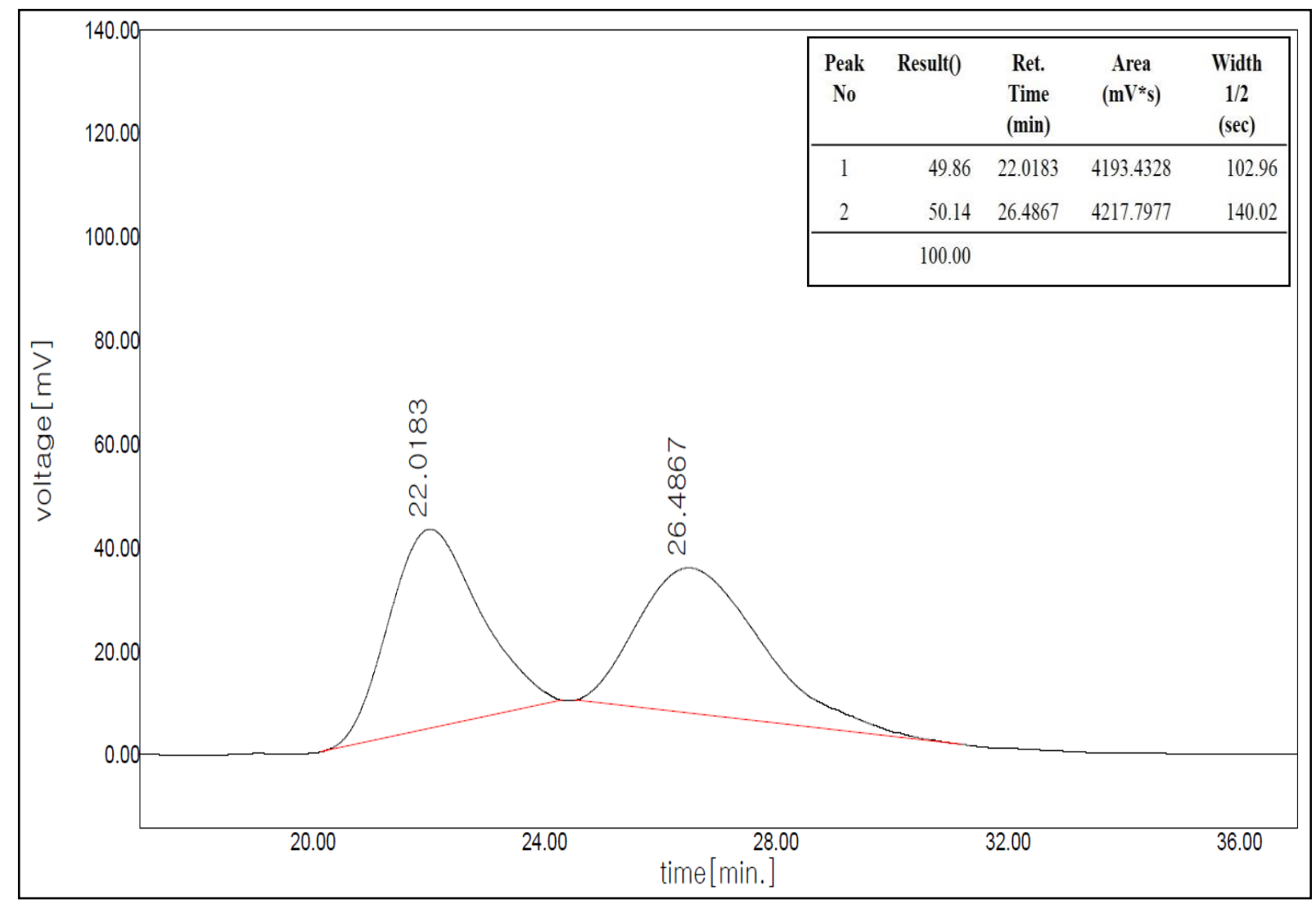

HPLC spectrum of $r a c-3 t$

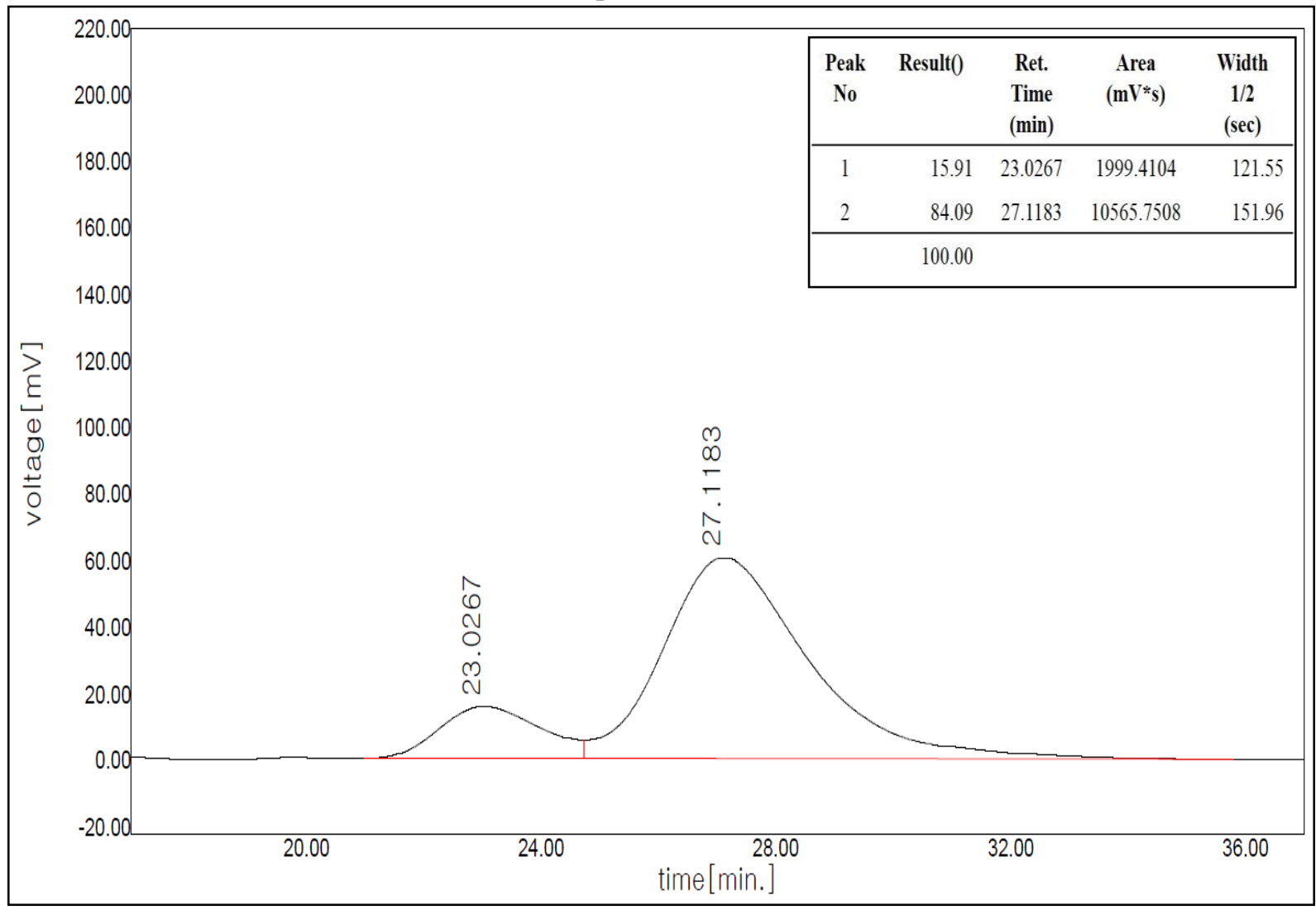

HPLC spectrum of $\mathbf{3 t}$ 


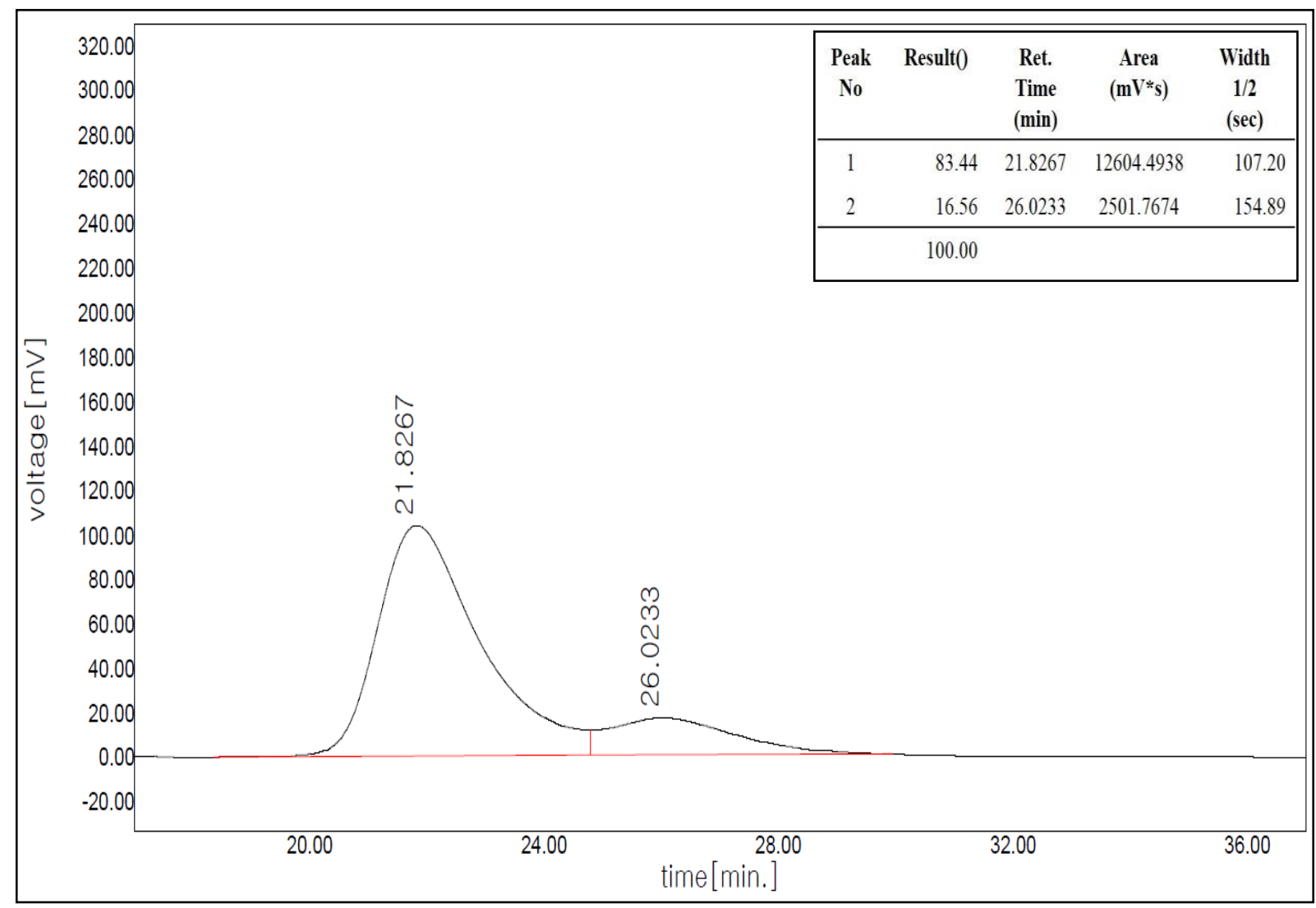

HPLC spectrum of ent-3t

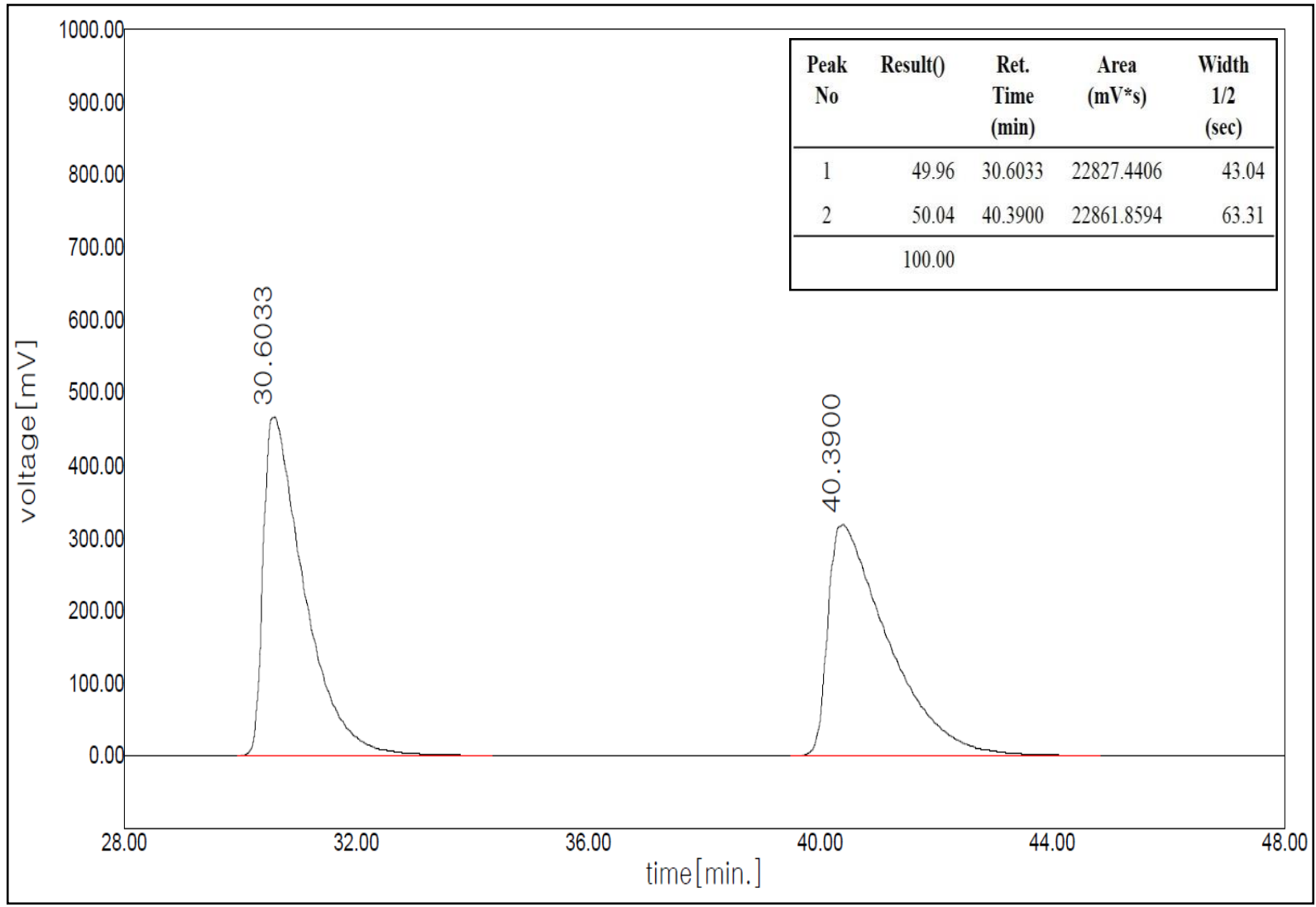

HPLC spectrum of $r a c-3 \mathbf{u}$ 


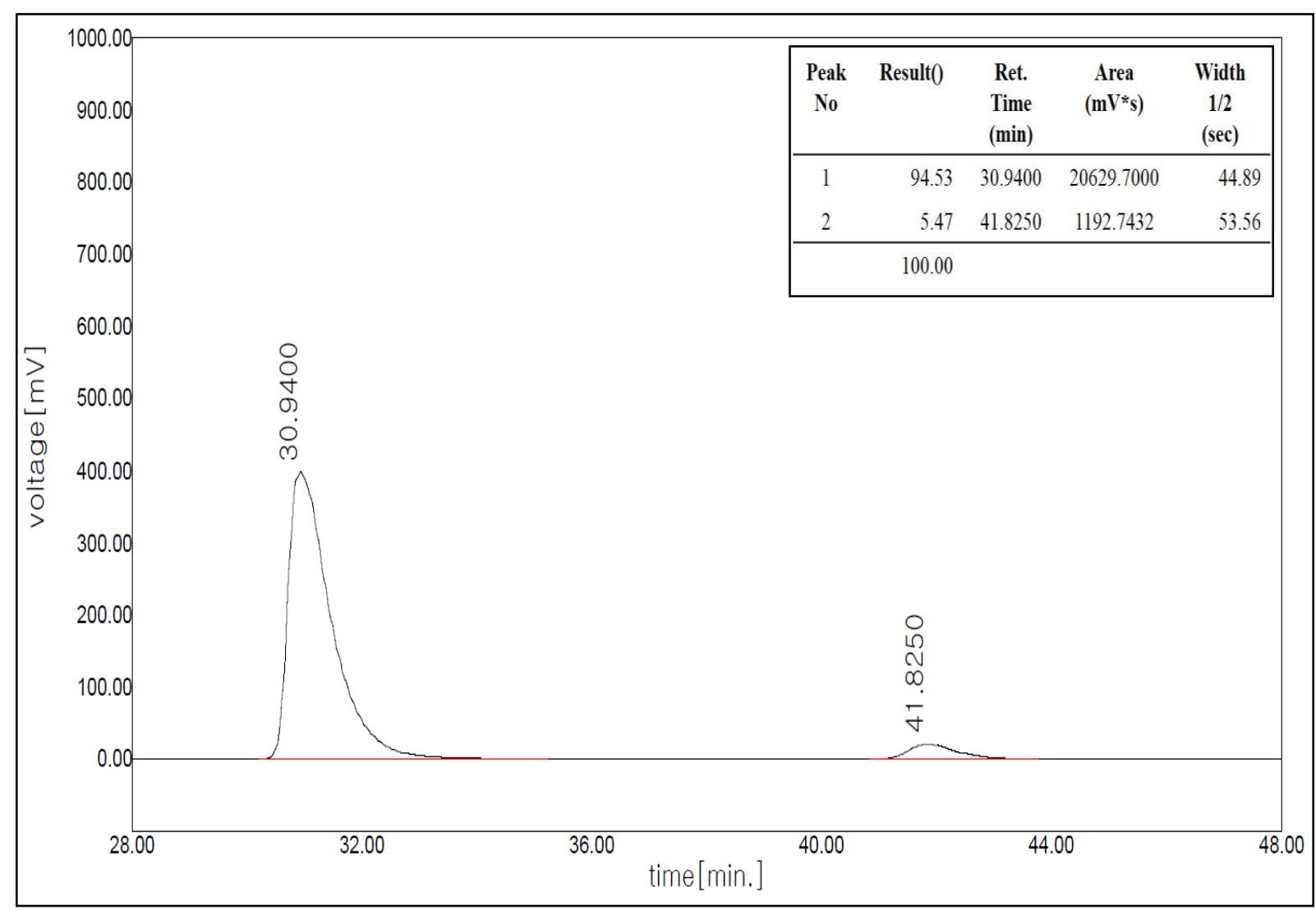

HPLC spectrum of $\mathbf{3 u}$

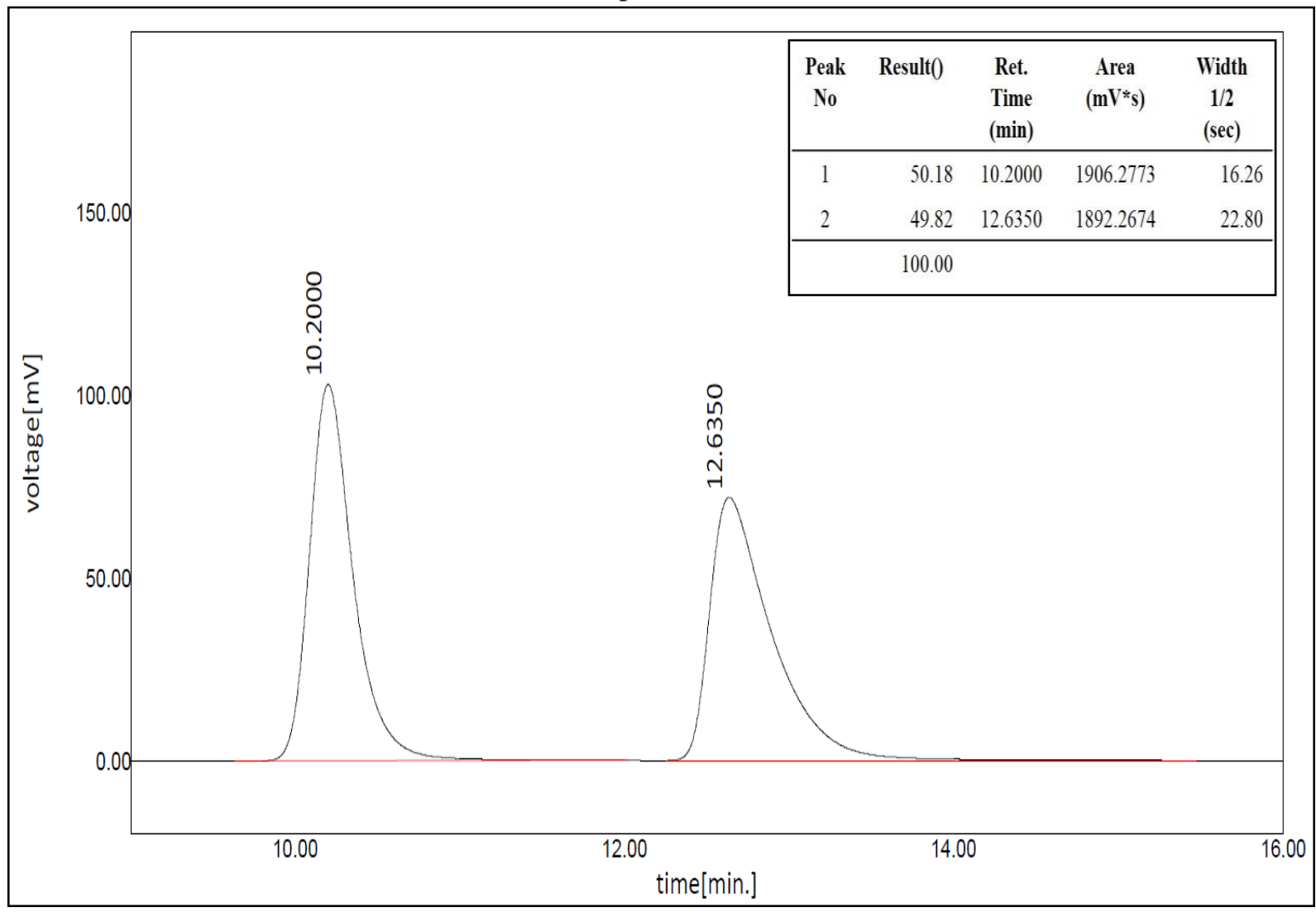

HPLC spectrum of $r a c-5 a$ 


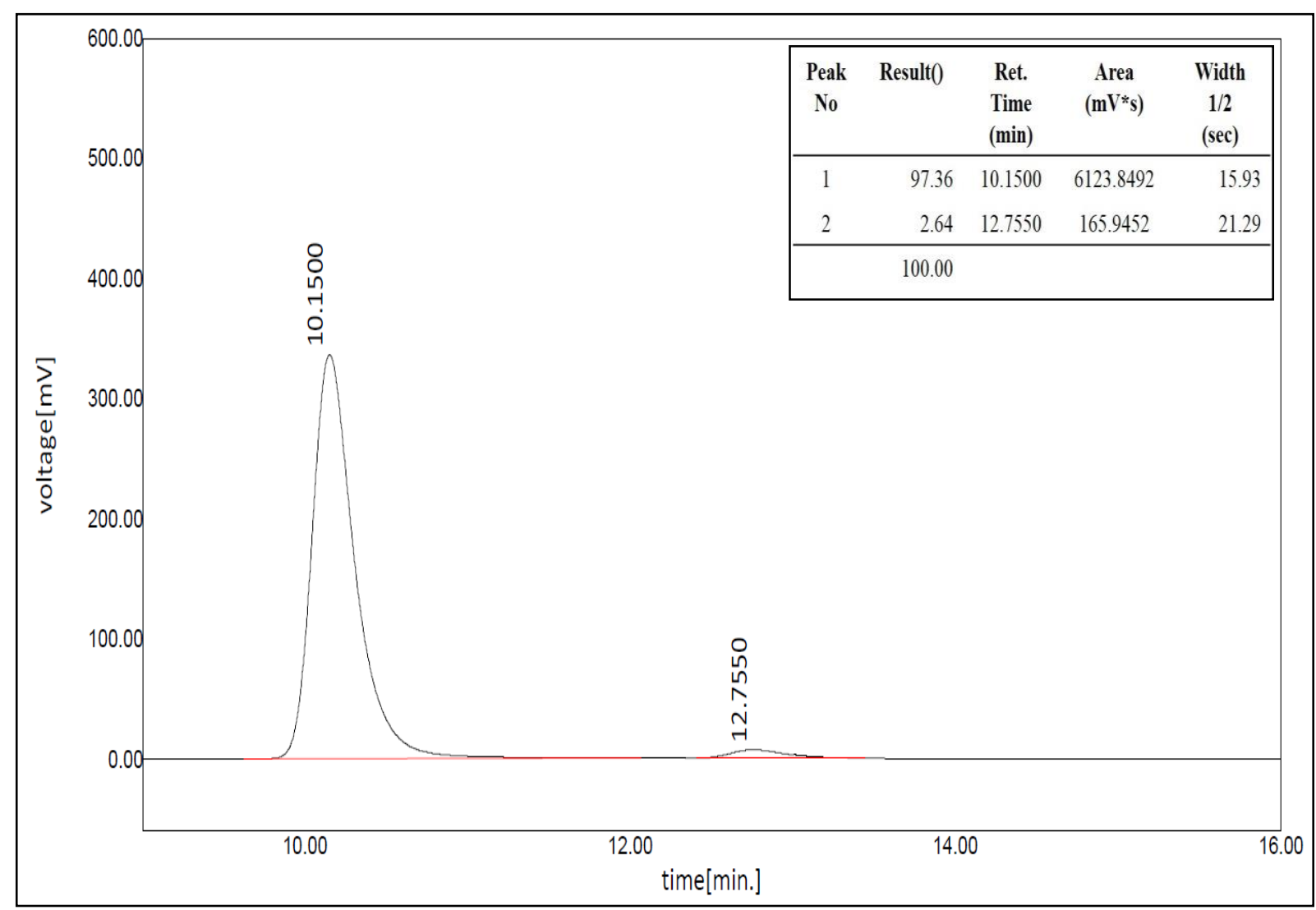

HPLC spectrum of $\mathbf{5 a}$

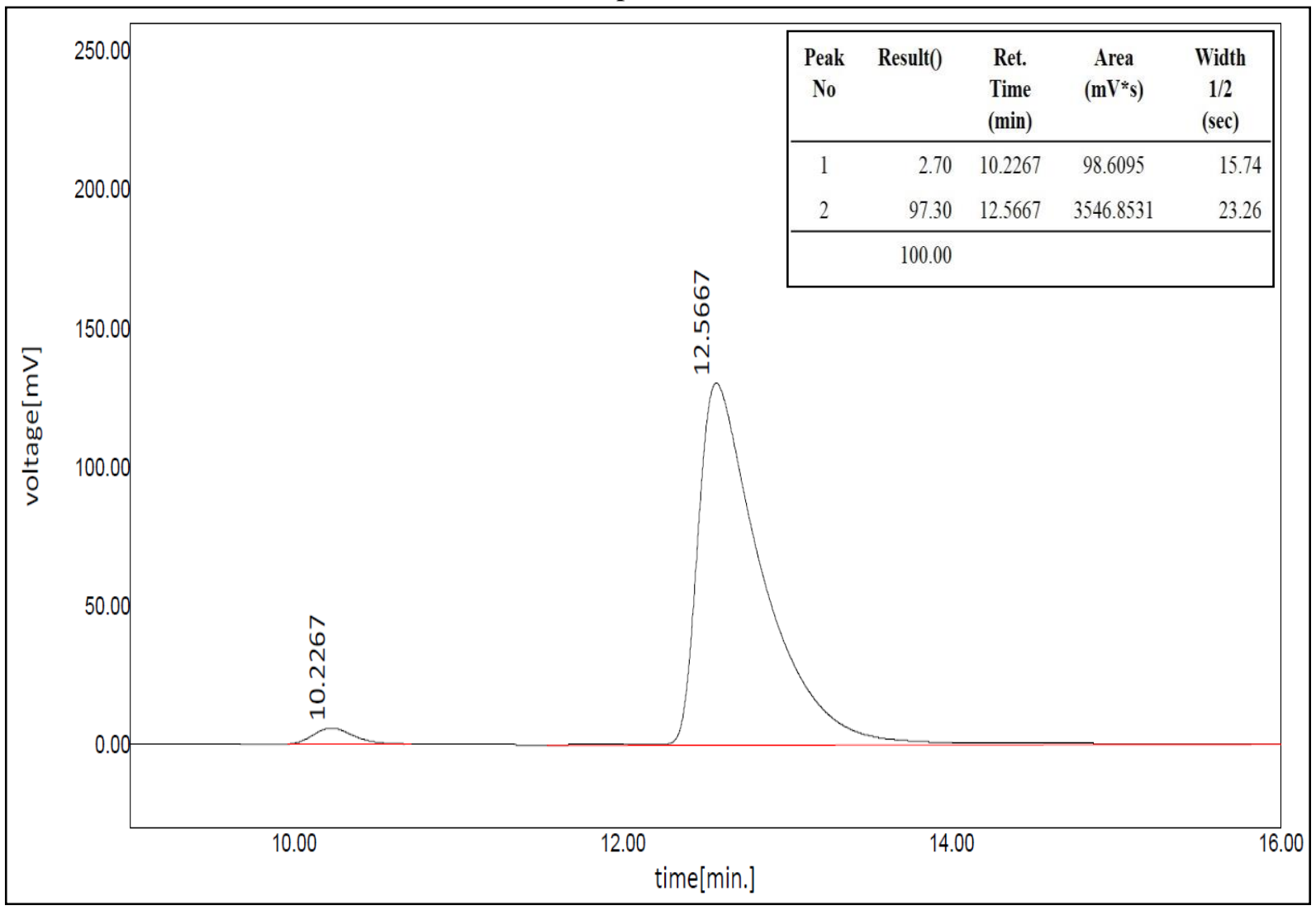

HPLC spectrum of ent-5a 


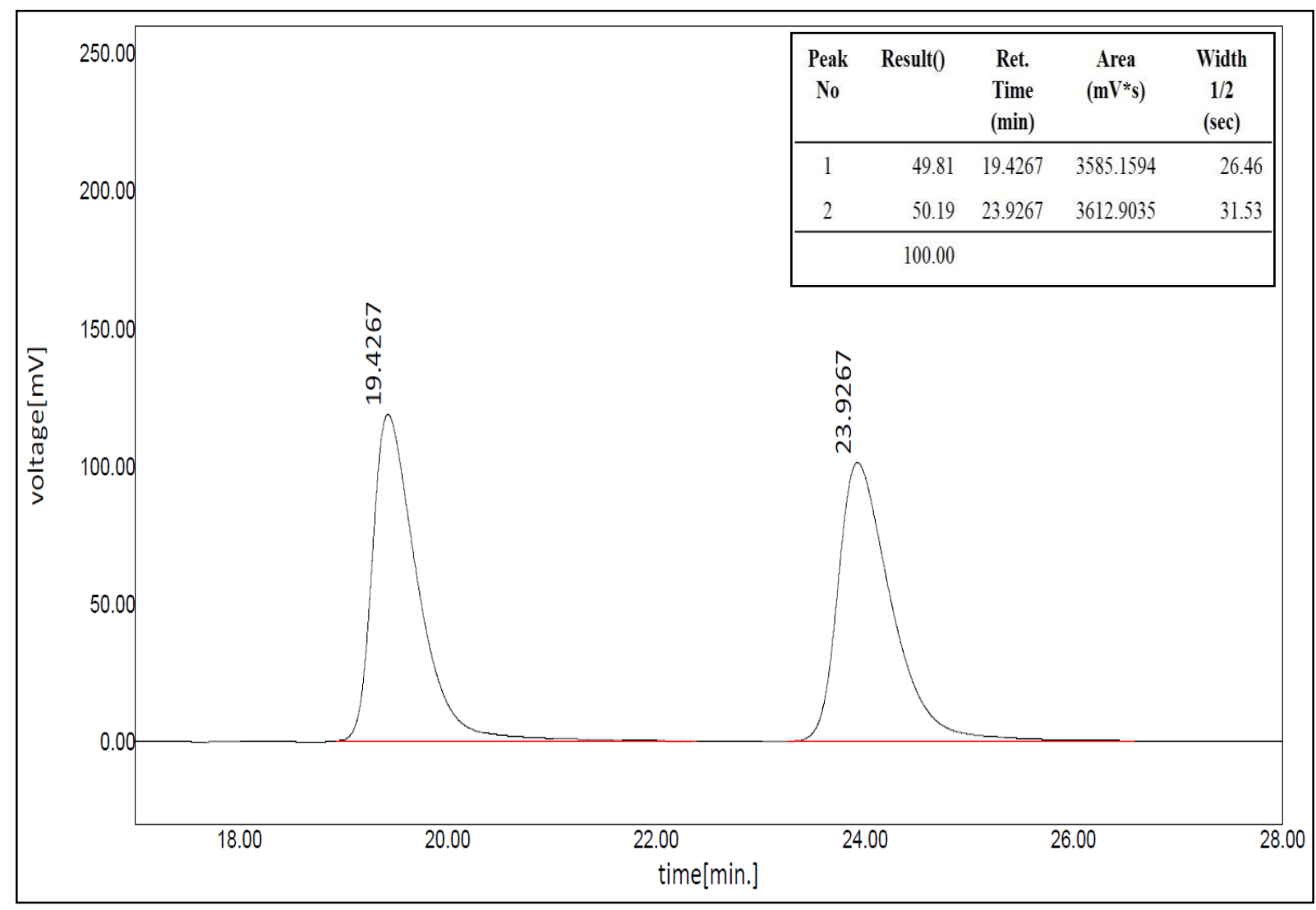

HPLC spectrum of $r a c-5 \mathbf{f}$

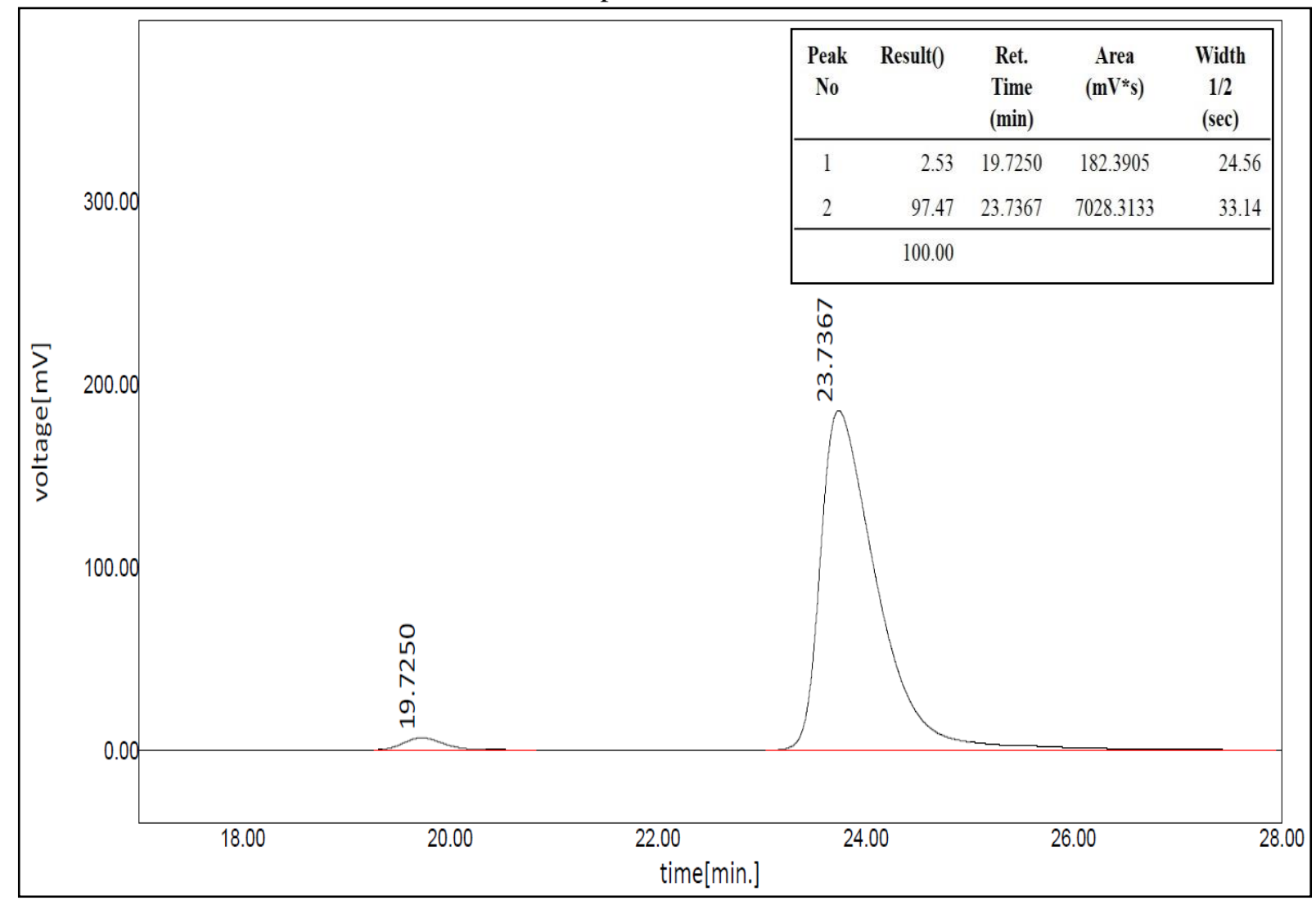

HPLC spectrum of $\mathbf{5 f}$ 


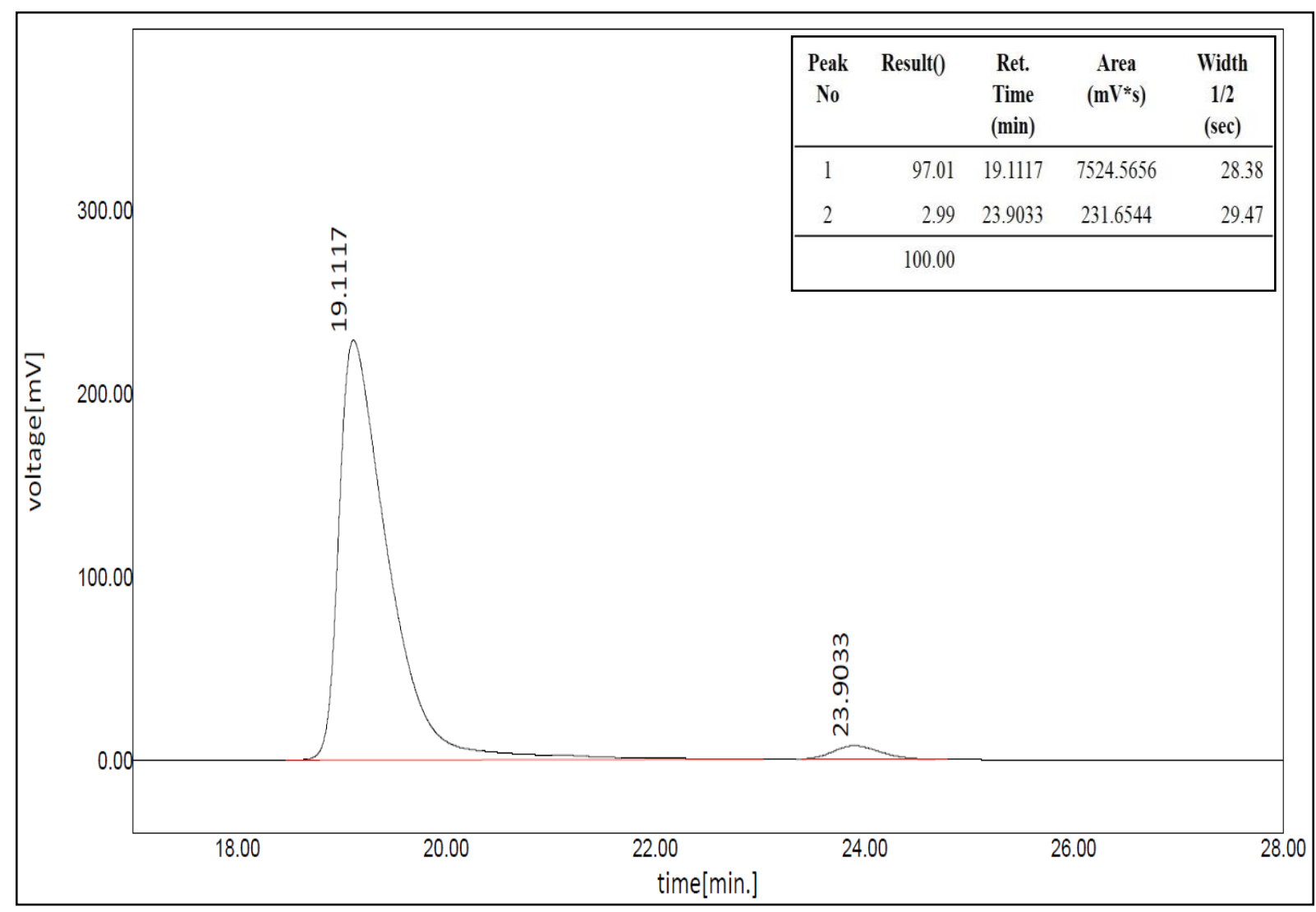

HPLC spectrum of ent-5f

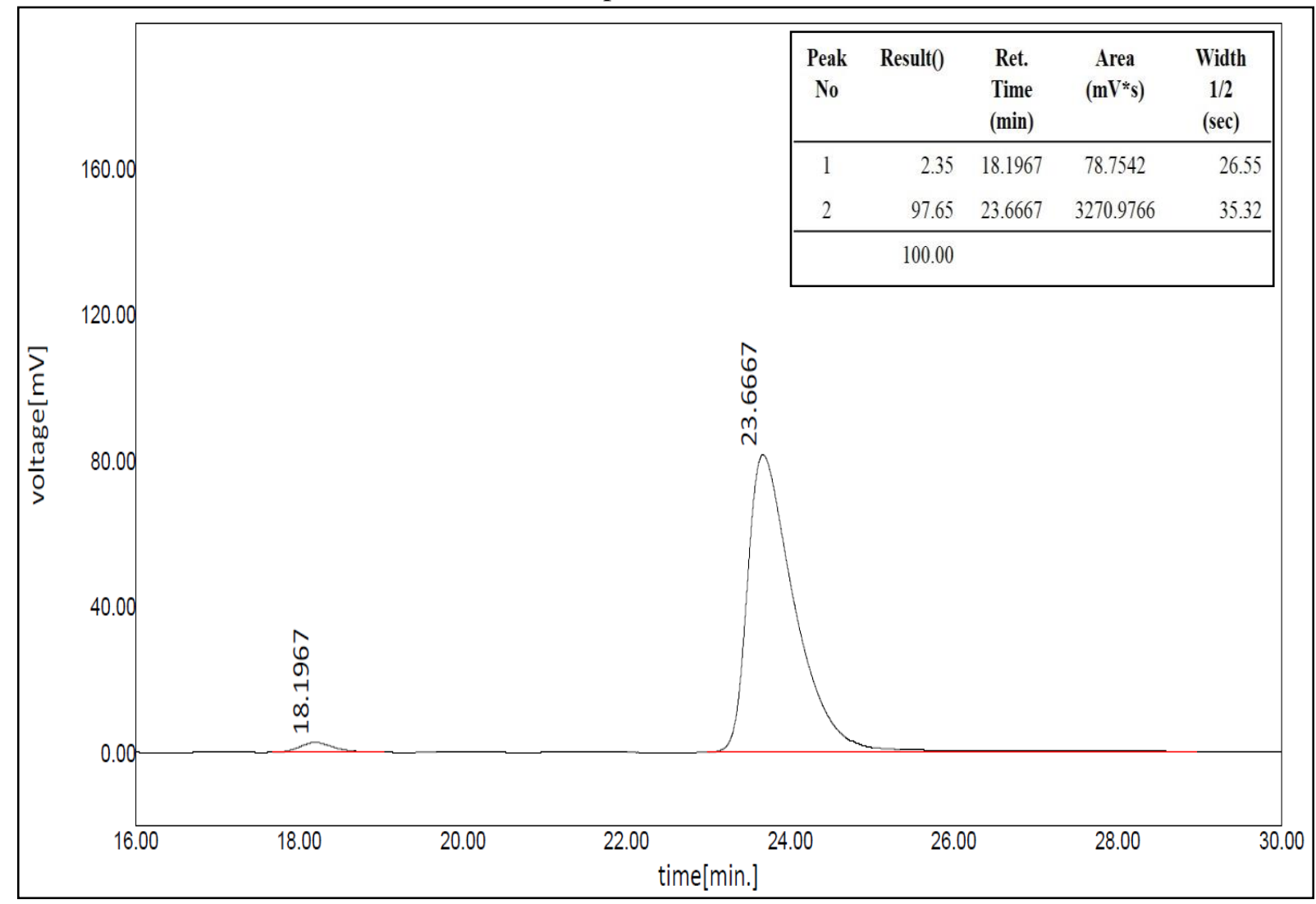

HPLC spectrum of $\mathbf{5 0}$ 


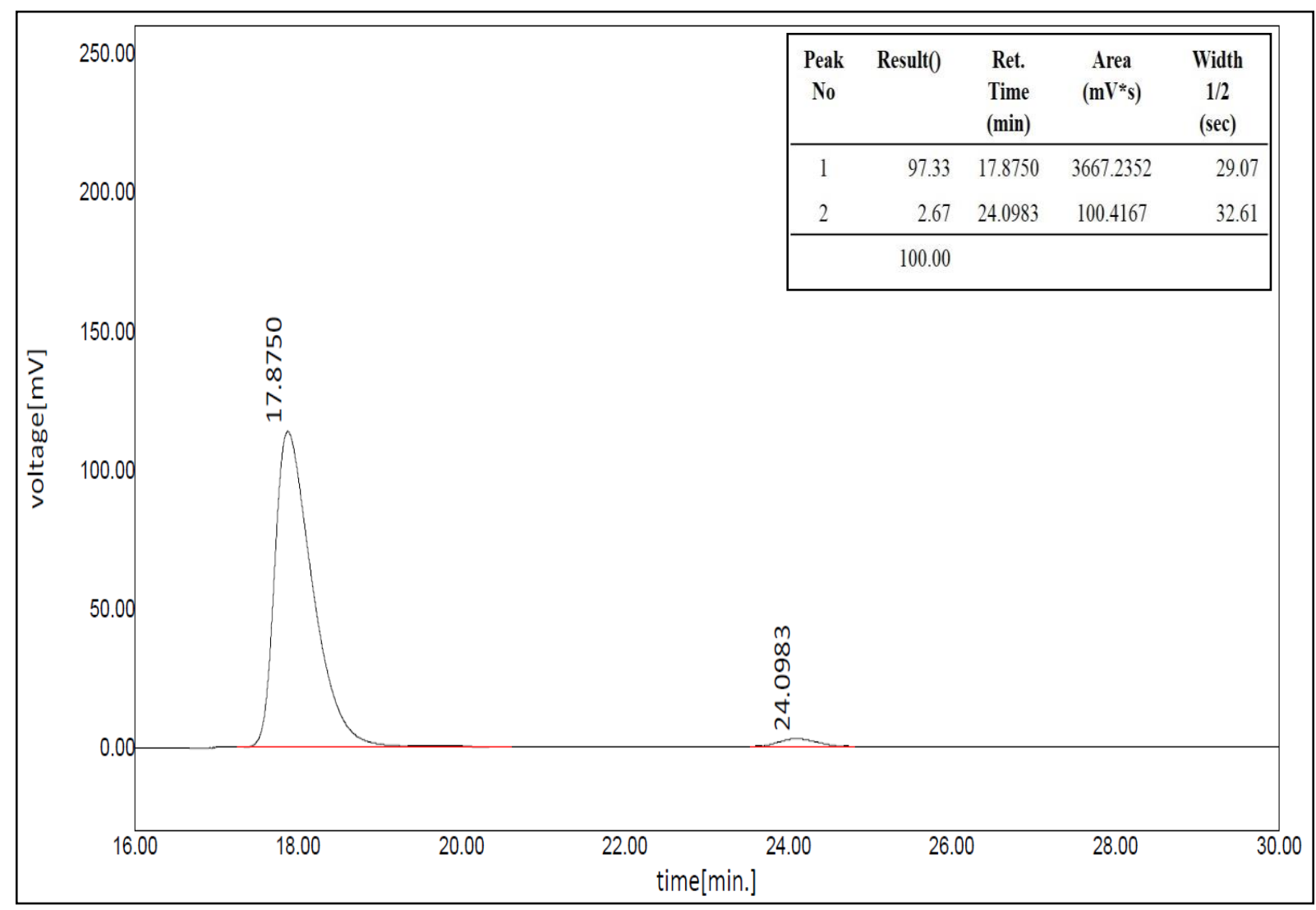

HPLC spectrum of ent-5o

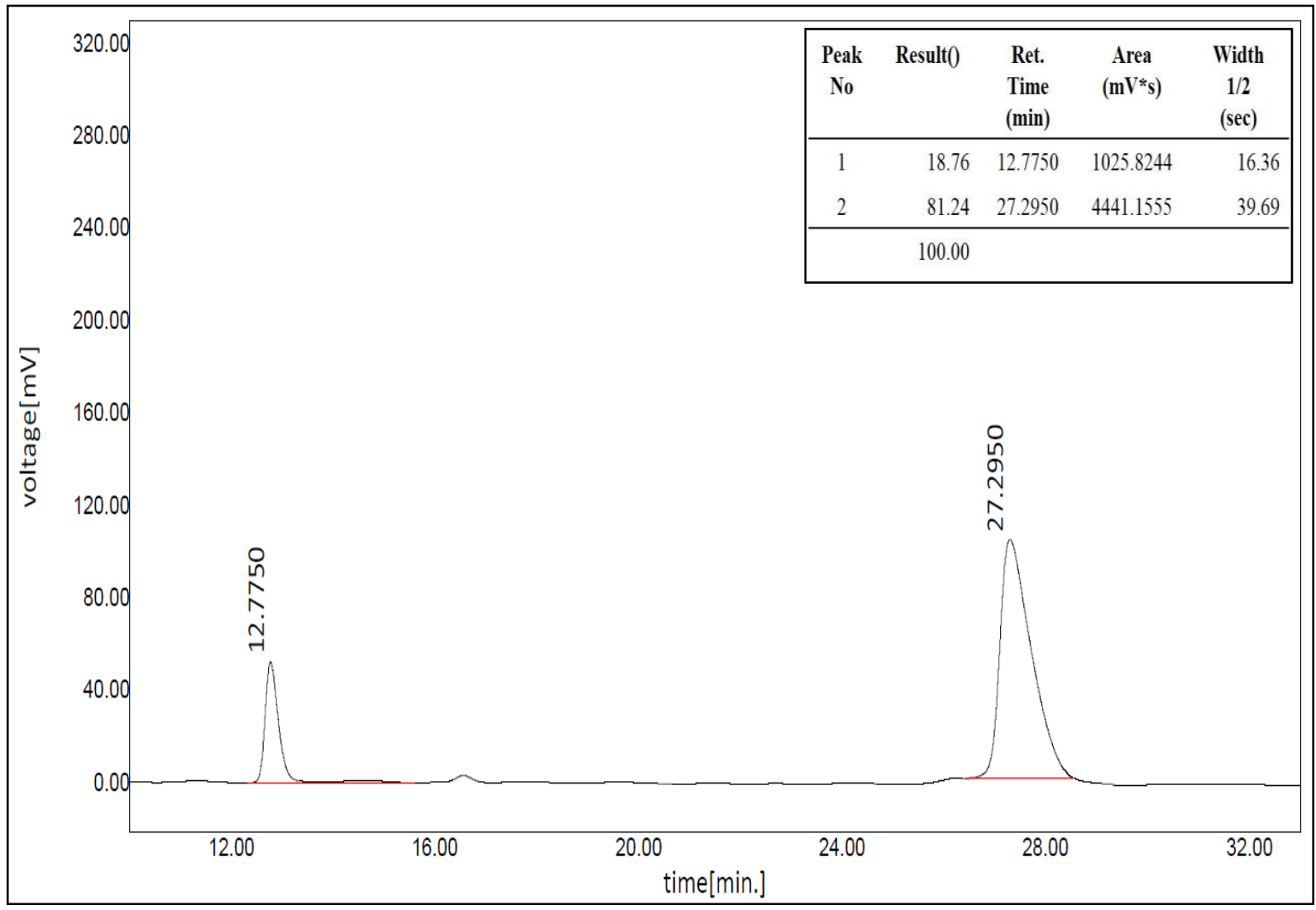

HPLC spectrum of $\mathbf{5 t}$ 


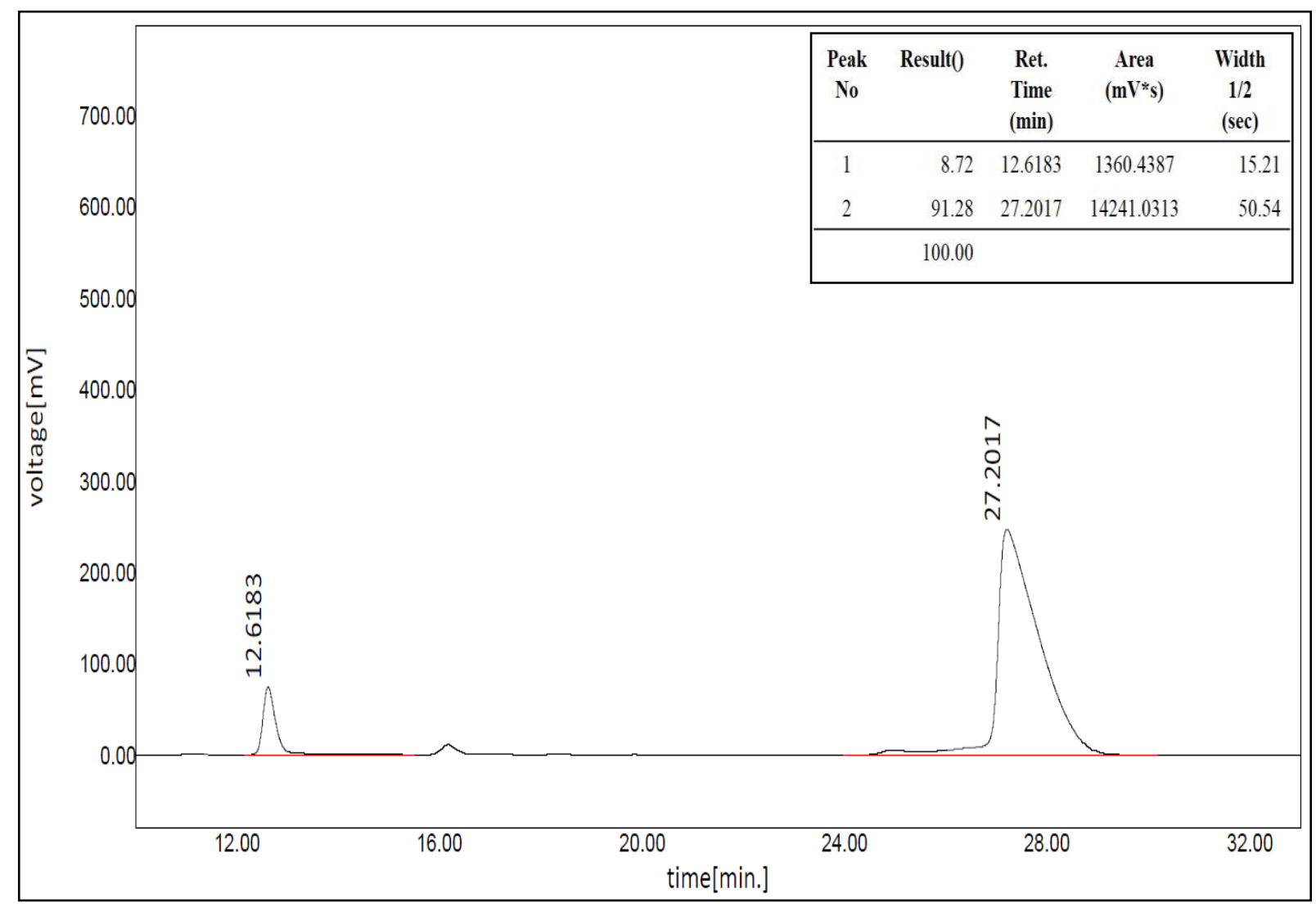

HPLC spectrum of $\mathbf{5 t}$

[after recrystallization from diethyl ether (enantioenriched product from filtrate)]

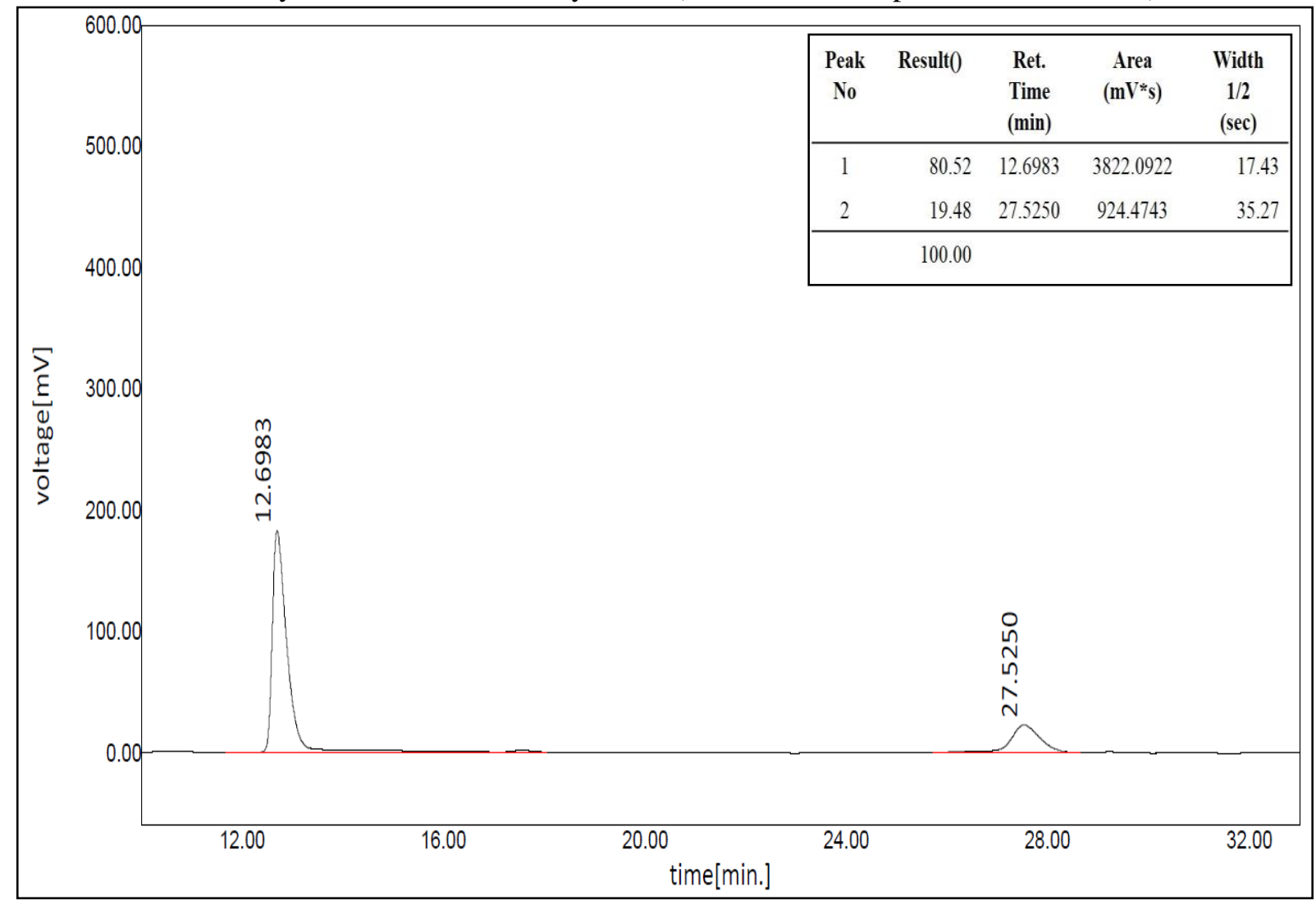

HPLC spectrum of ent-5t 


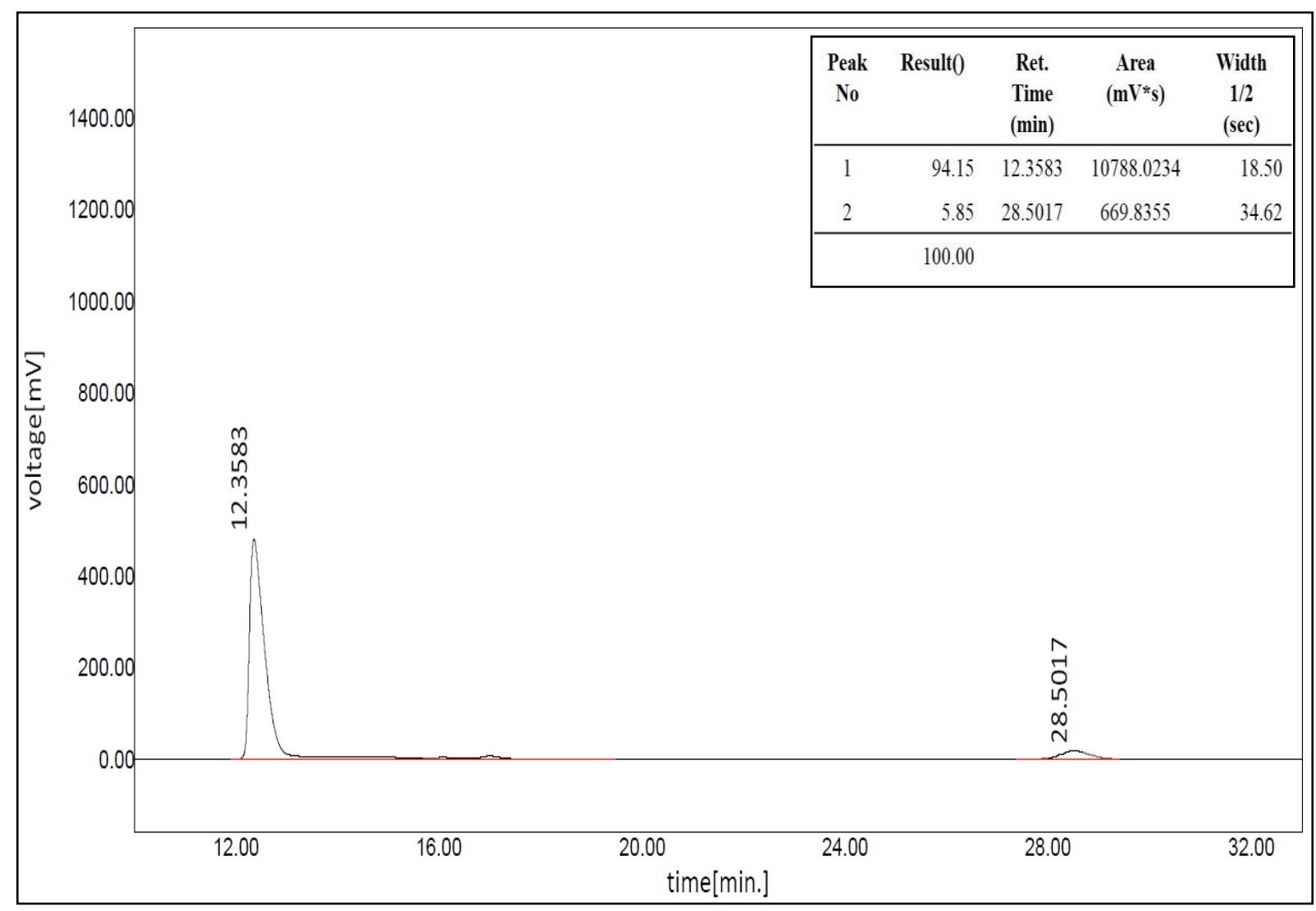

HPLC spectrum of ent-5t

[after recrystallization from diethyl ether (enantioenriched product from filtrate)]

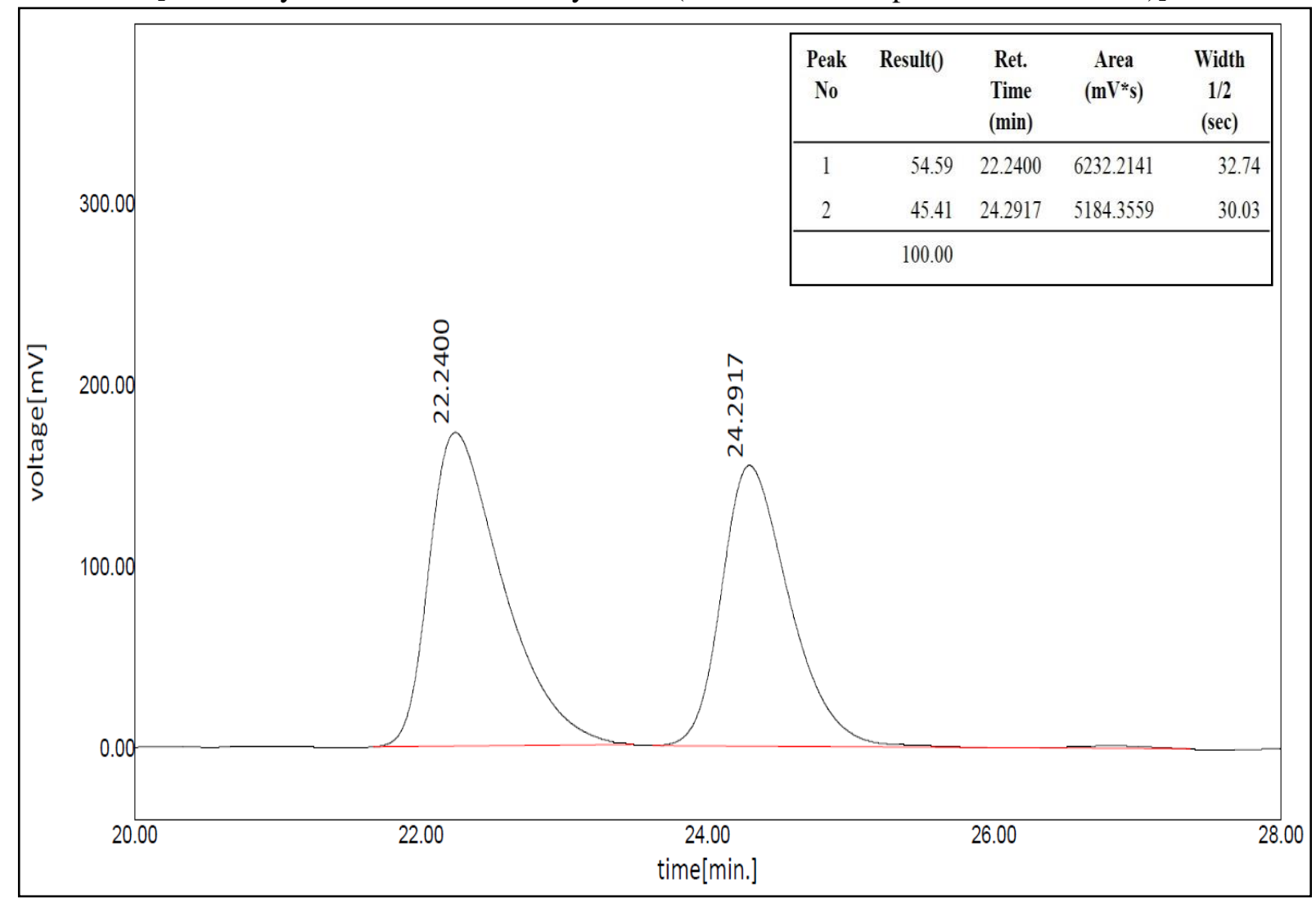

HPLC spectrum of $\mathrm{rac}-\mathbf{6 a}$ 


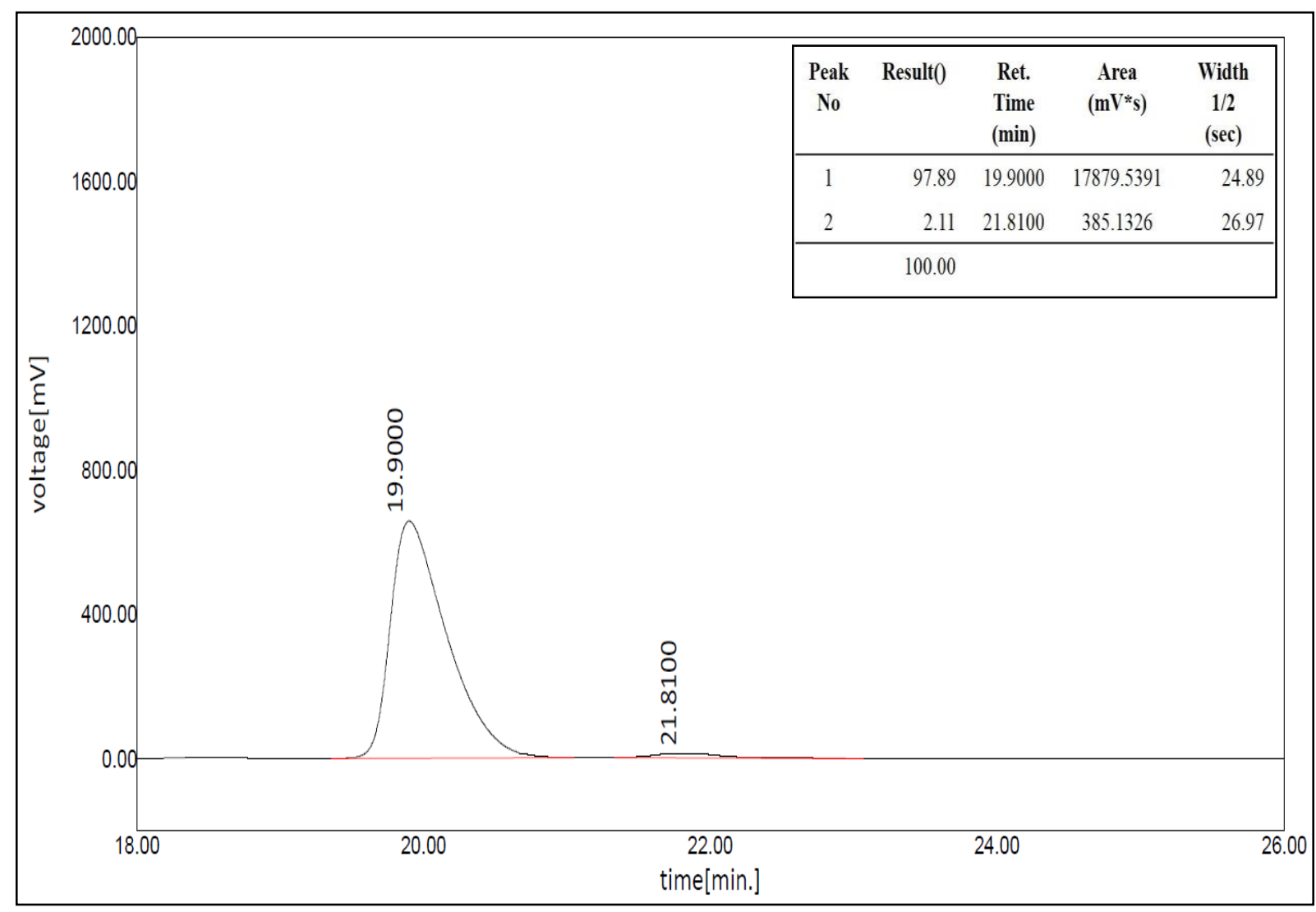

HPLC spectrum of $\mathbf{6 a}$

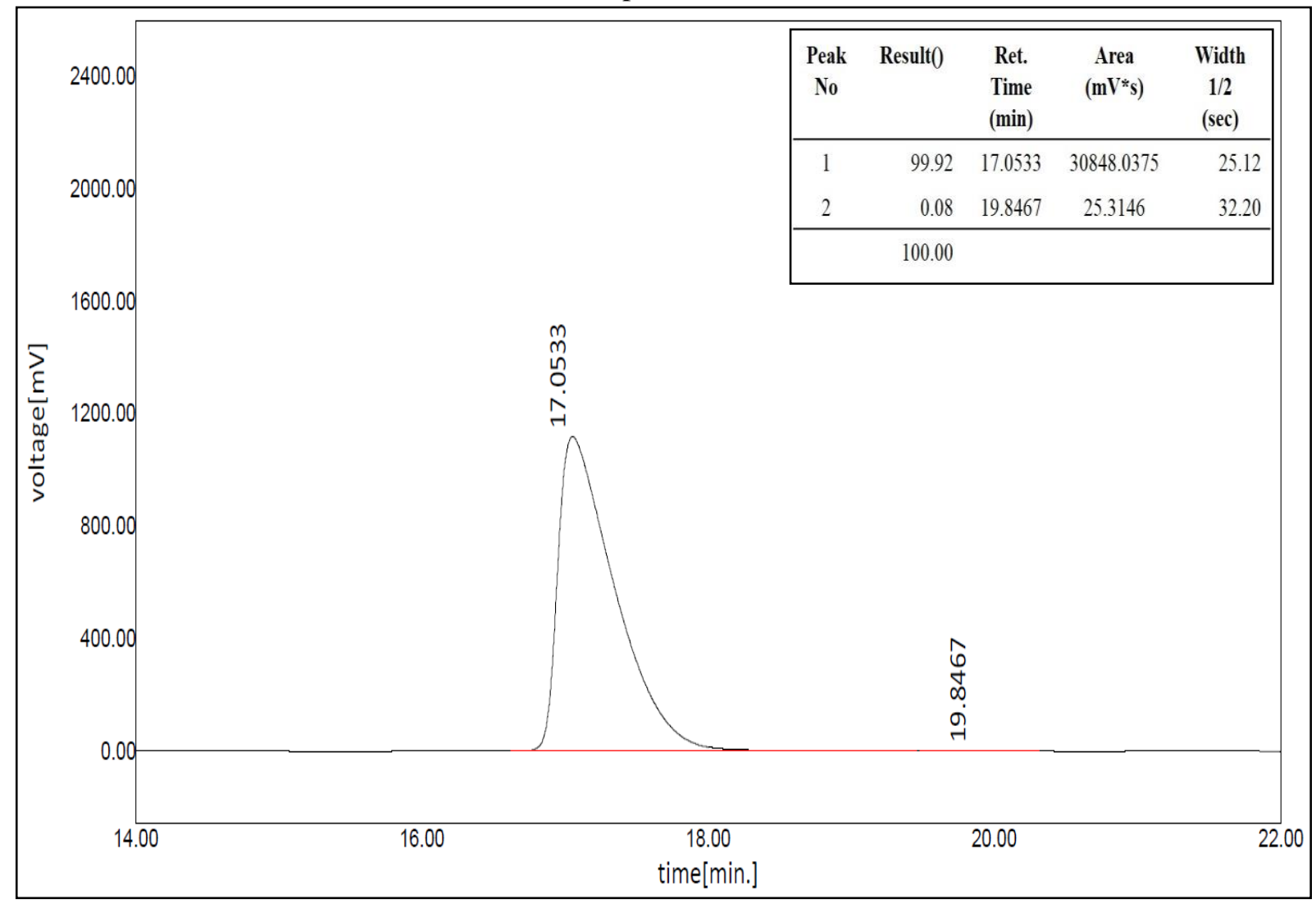

HPLC spectrum of $\mathbf{6 a}$

[after recrystallization from methyl tert-butyl ether] 


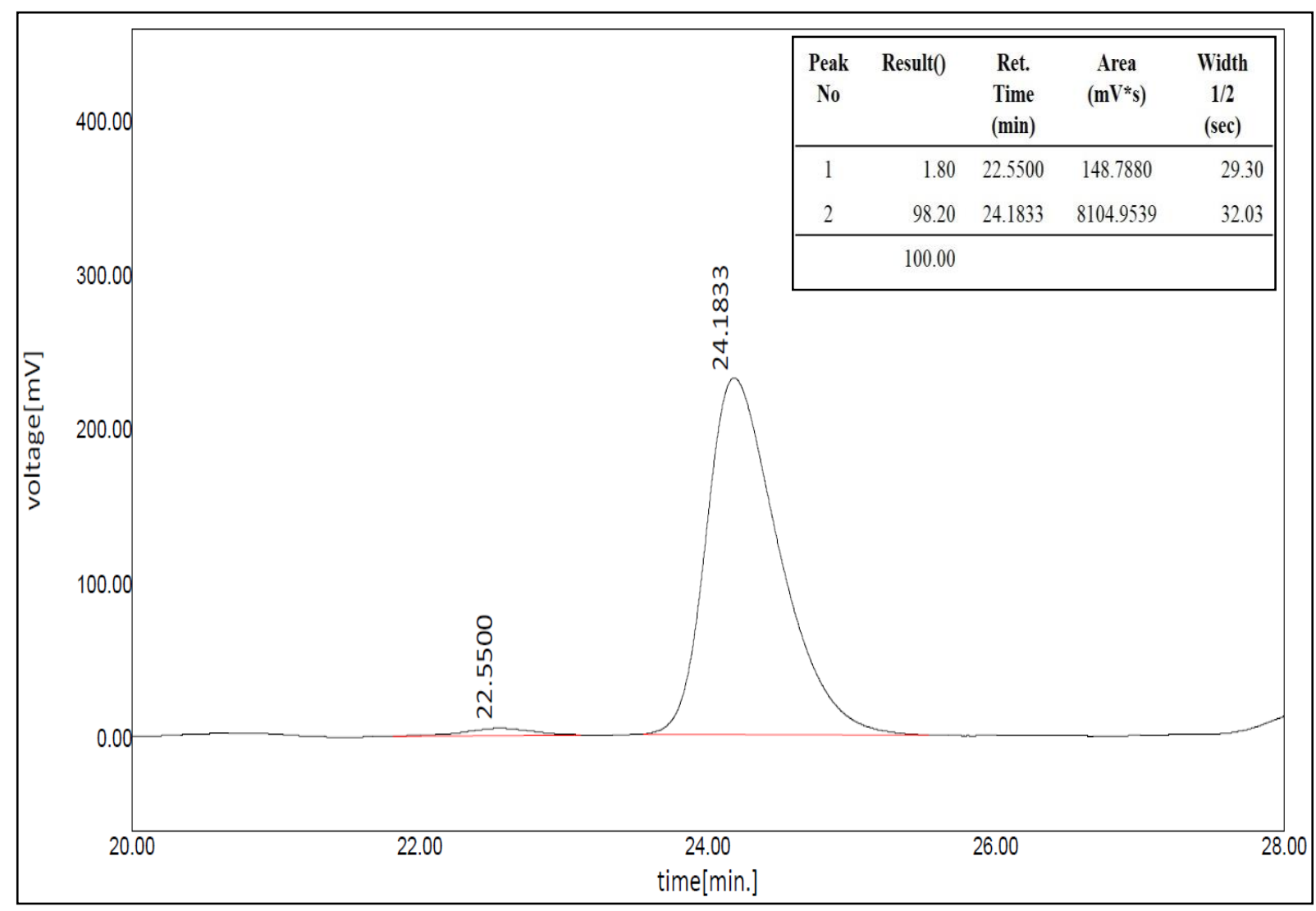

HPLC spectrum of ent-6a

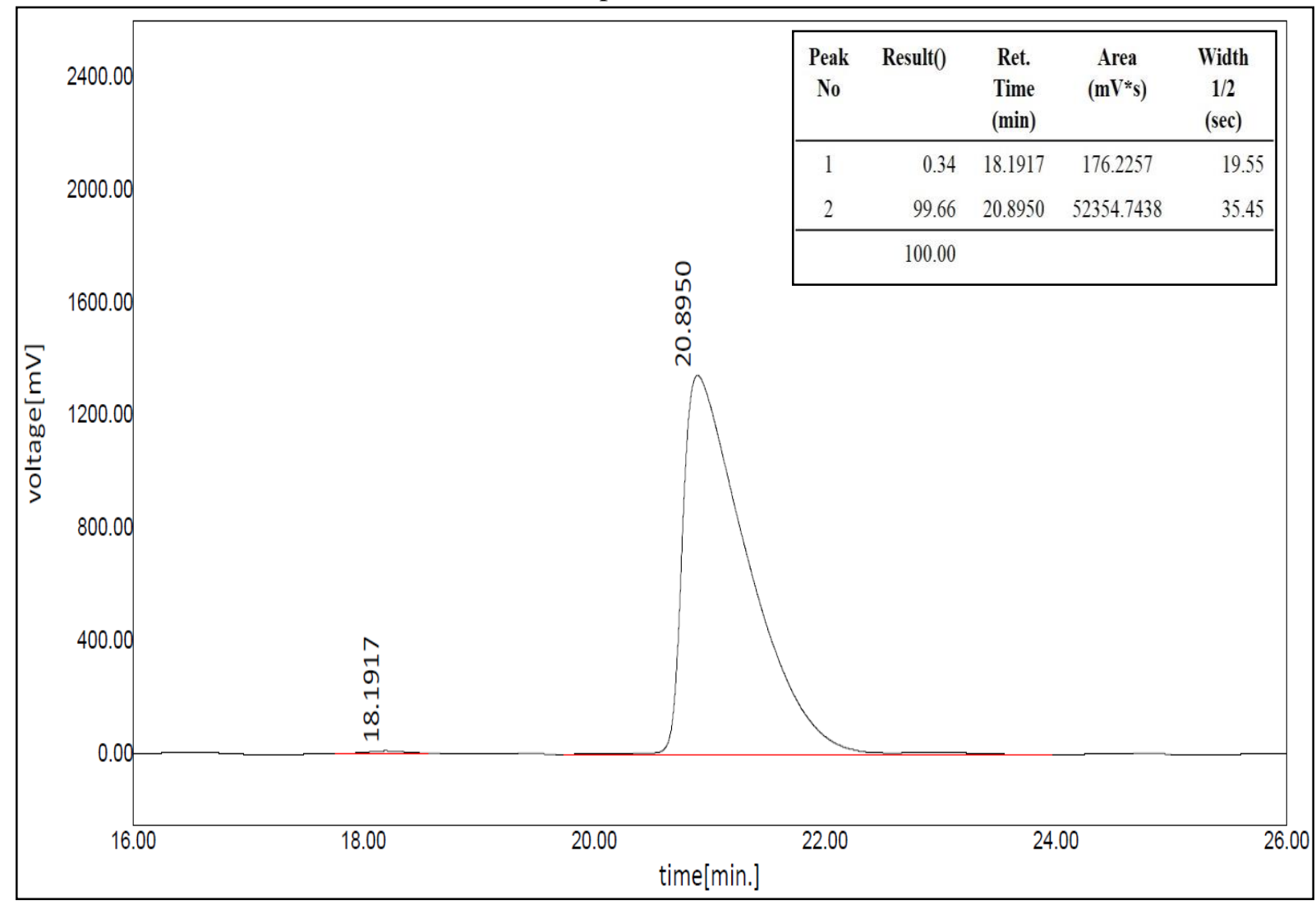

HPLC spectrum of ent-6a

[after recrystallization from methyl tert-butyl ether] 


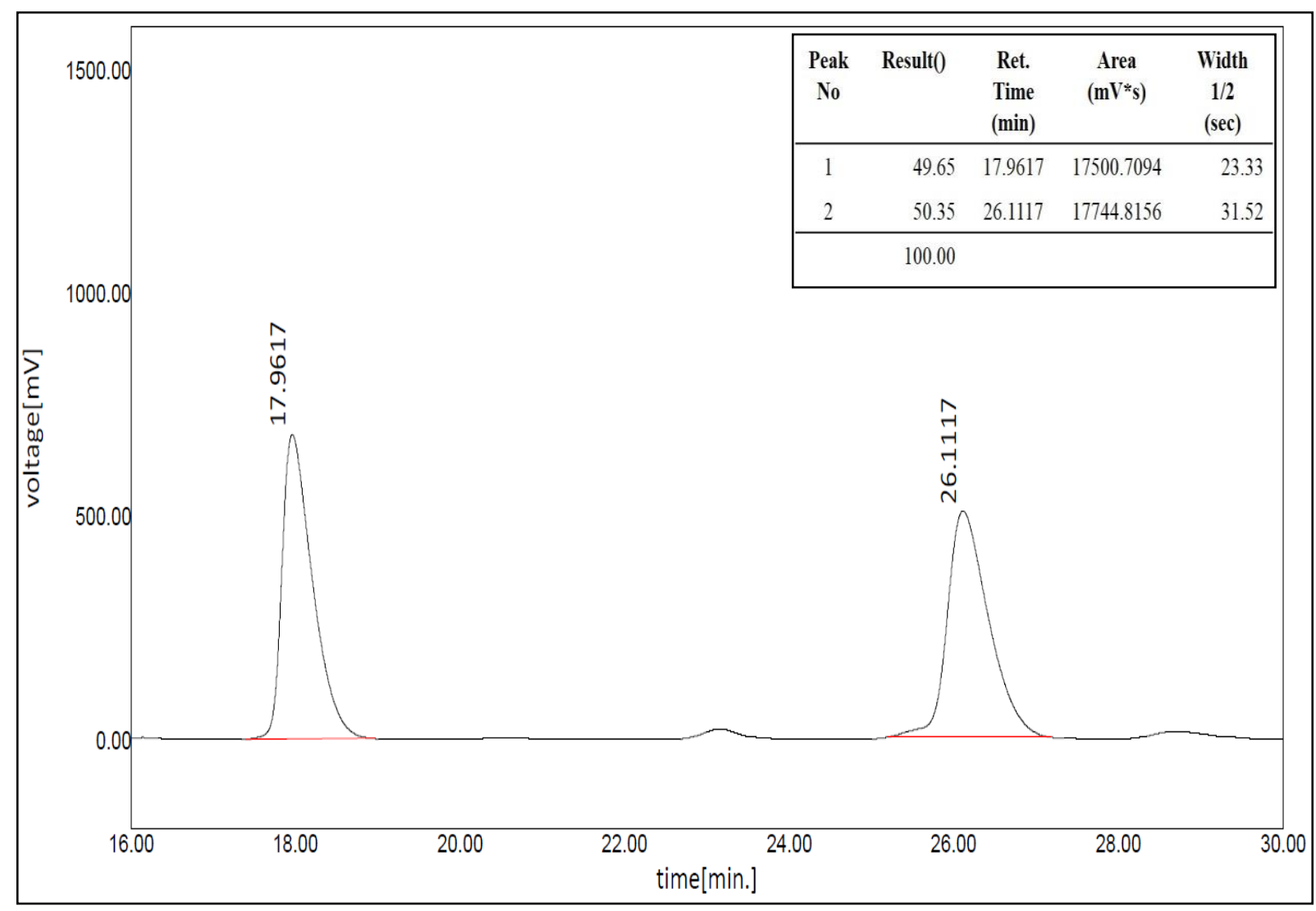

HPLC spectrum of $r a c-6 \mathbf{f}$

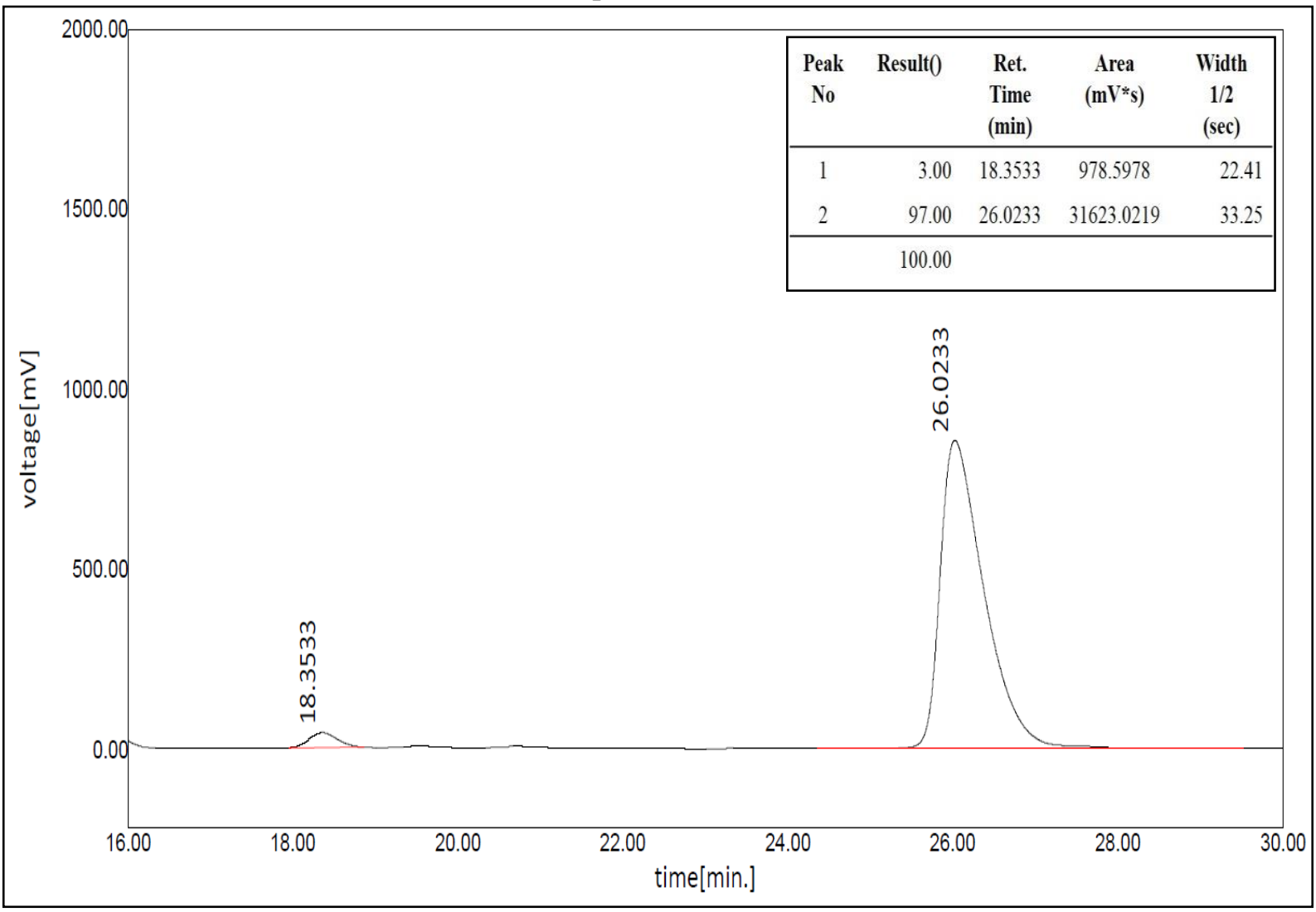

HPLC spectrum of $\mathbf{6 f}$ 


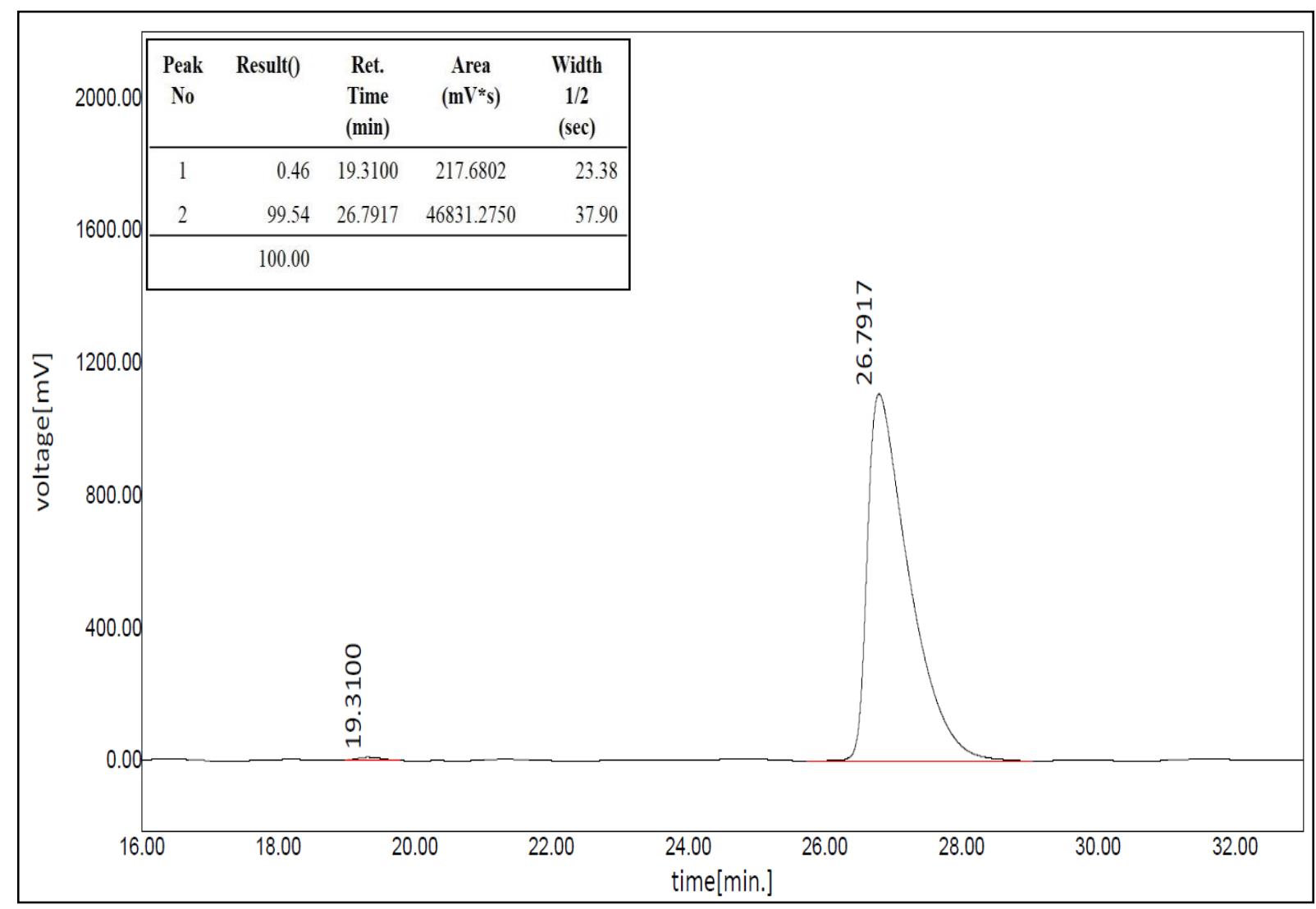

HPLC spectrum of $\mathbf{6 f}$

[after recrystallization from methyl tert-butyl ether]

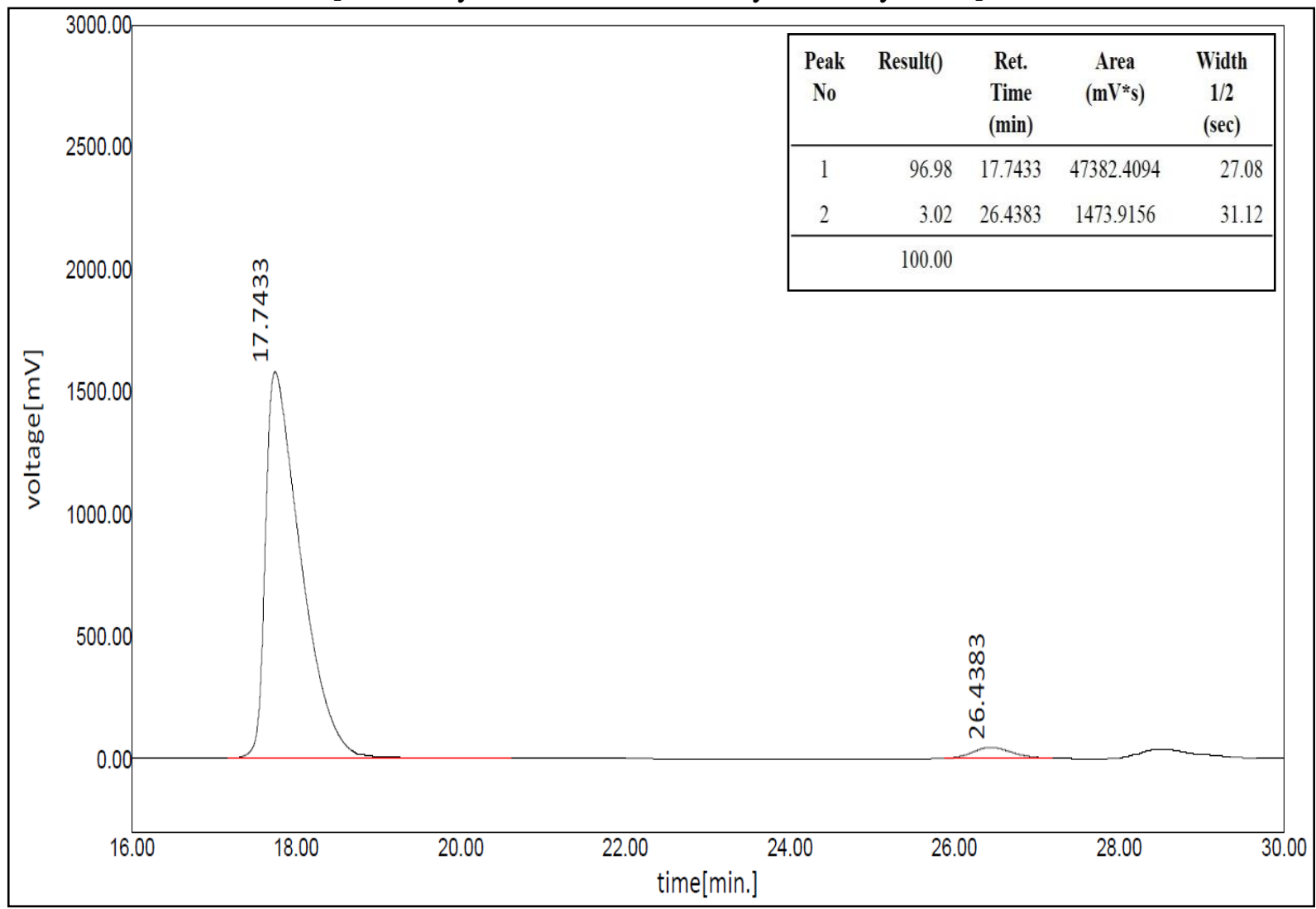

HPLC spectrum of ent-6f 


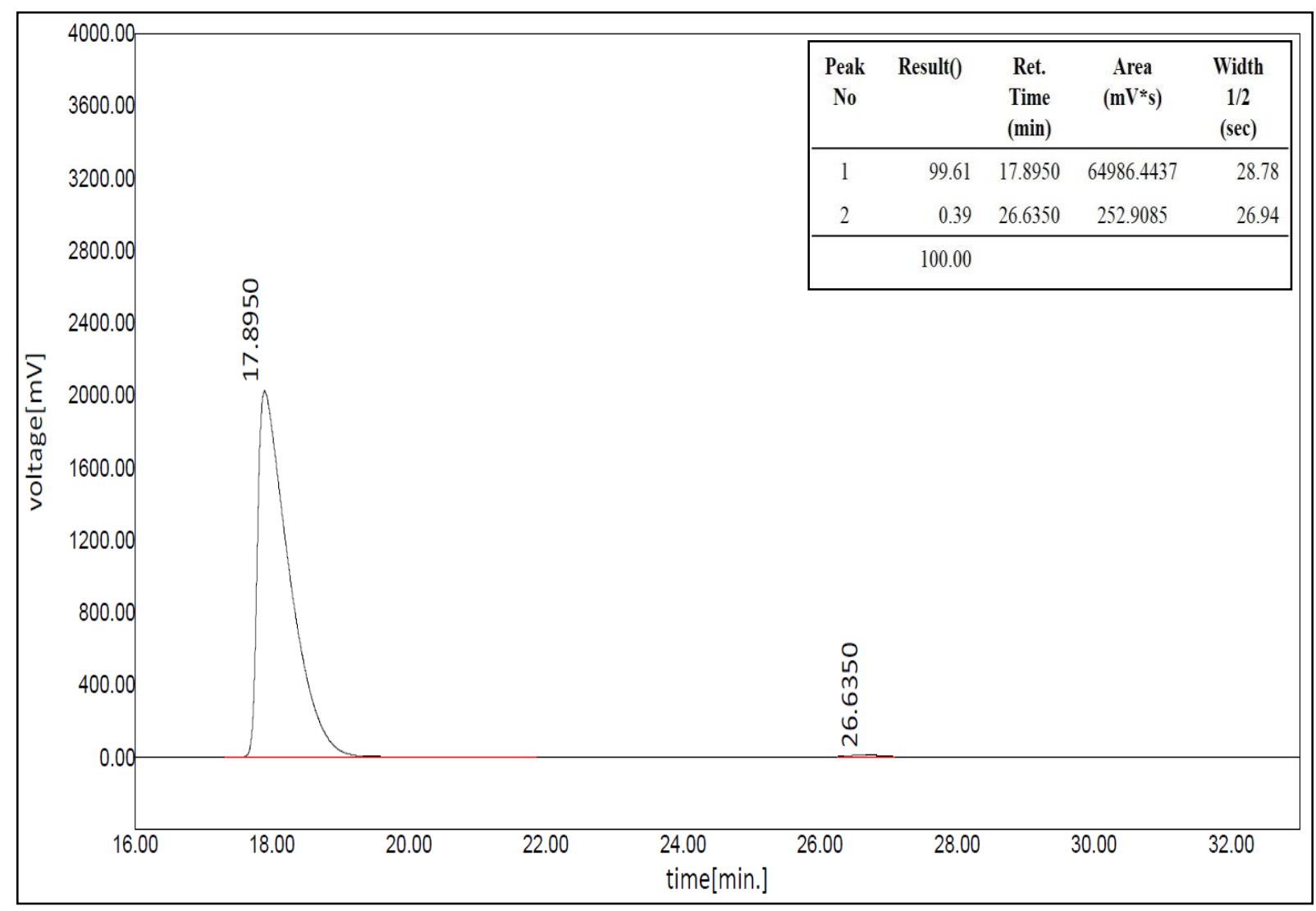

HPLC spectrum of ent-6f

[after recrystallization from methyl tert-butyl ether]

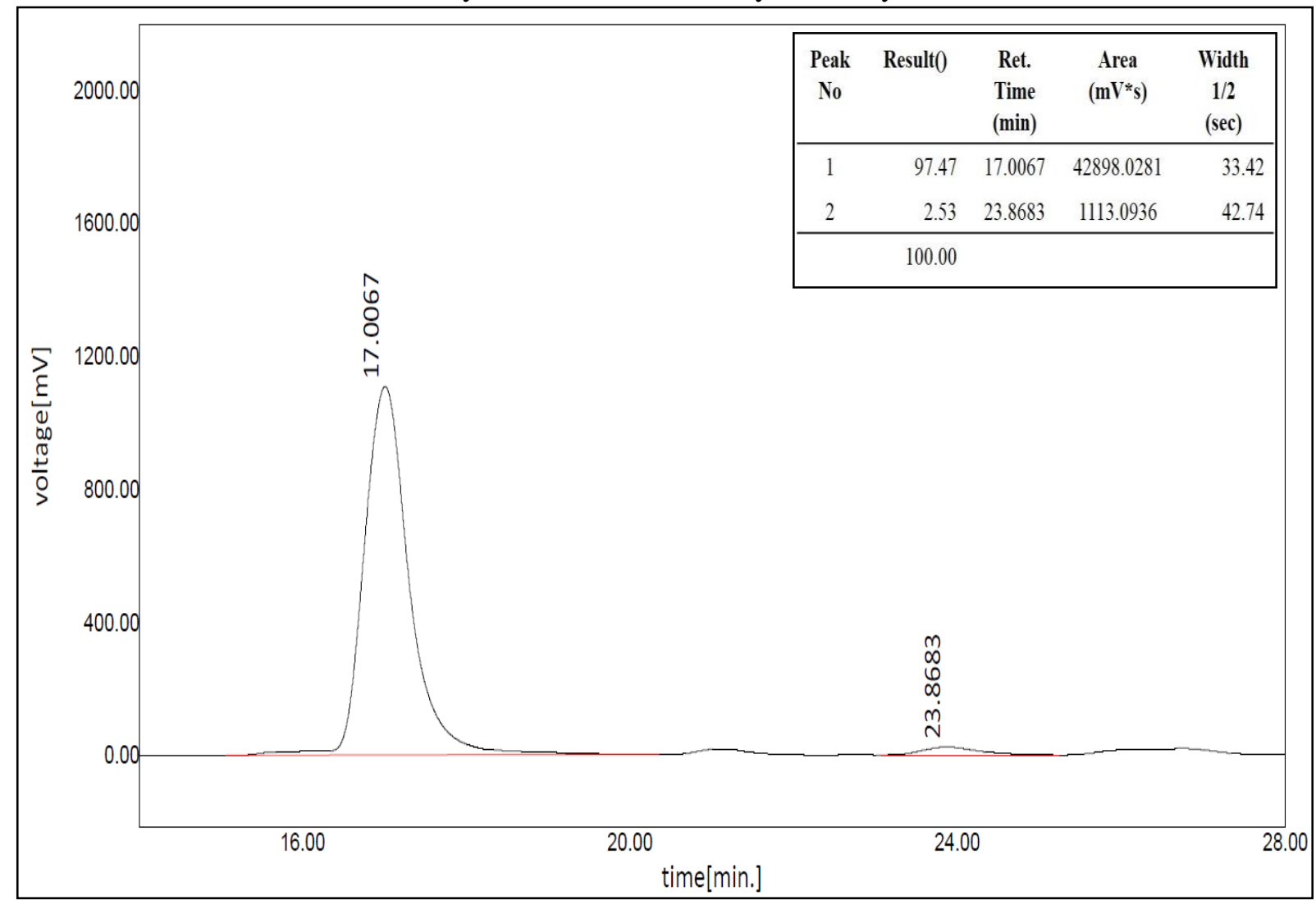

HPLC spectrum of $\mathbf{6 0}$ 


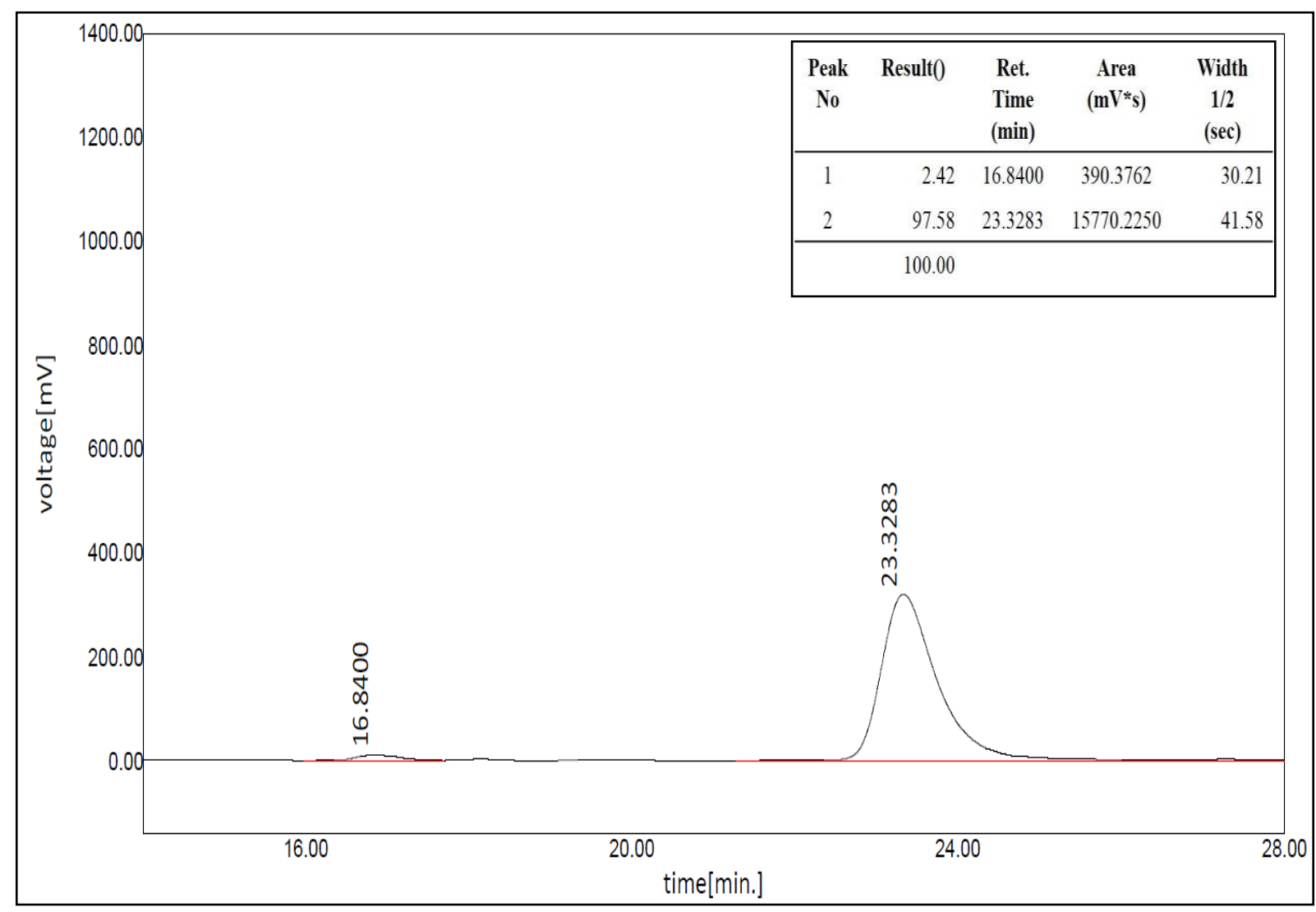

HPLC spectrum of ent-6o

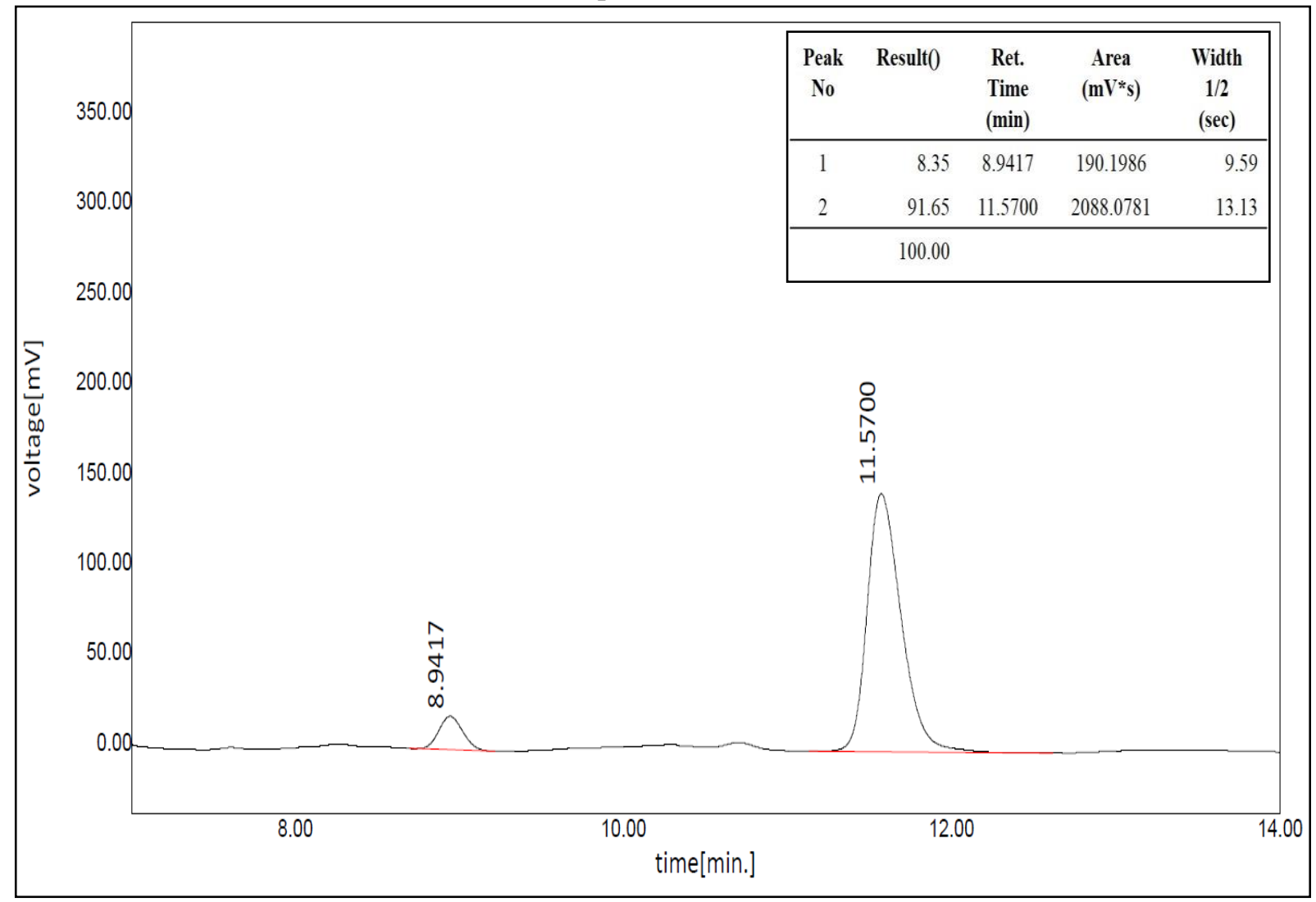

HPLC spectrum of $\mathbf{6 t}$ 


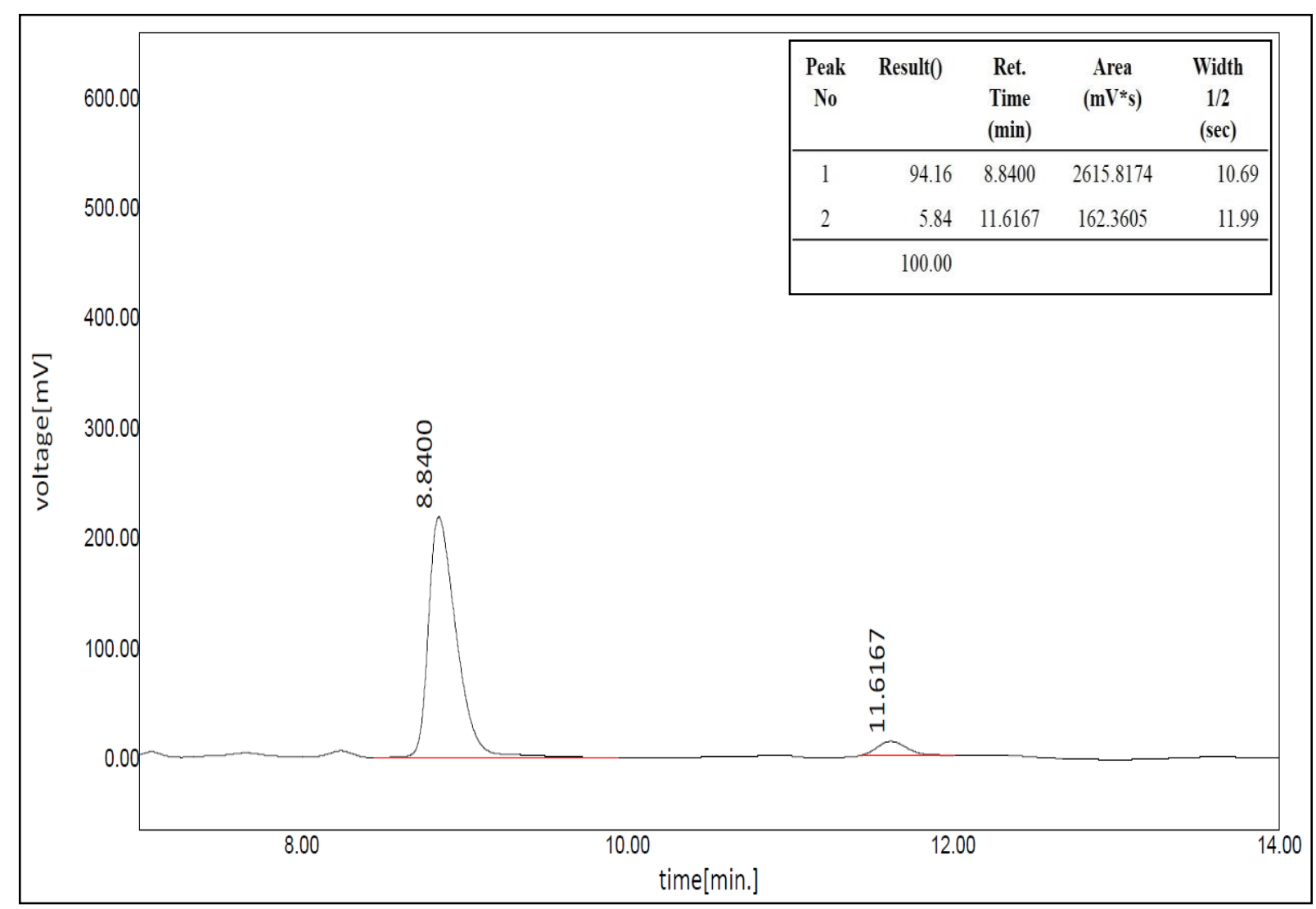

HPLC spectrum of ent-6t 


\section{Single crystal X-ray structure analysis of ent-3ab (CCDC 1920284)}

\section{* X-ray structure analysis parameters for ent-3ab}

Identification code

Empirical formula

Formula weight

Temperature

Wavelength

Crystal system

Space group

Unit cell dimensions

Volume

Z

Density (calculated)

Absorption coefficient

$\mathrm{F}(000)$

Crystal size

Theta range for data collection

Index ranges

Reflections collected

Independent reflections

Completeness to theta $=25.242^{\circ}$

Absorption correction

Max. and min. transmission

Refinement method

Data / restraints / parameters

Goodness-of-fit on $\mathrm{F}^{2}$

Final $\mathrm{R}$ indices [I>2sigma(I)]

$\mathrm{R}$ indices (all data)

Absolute structure parameter

Extinction coefficient

Largest diff. peak and hole
SJH1600

C26 H22 F3 N O4 S2

533.56

223(2) K

$0.71073 \AA$

Monoclinic

$\mathrm{P} 2_{1}$

$\mathrm{a}=12.4410(6) \AA \quad \alpha=90^{\circ}$.

$\mathrm{b}=8.8810(4) \AA \quad \beta=115.9116(16)^{\circ}$.

$\mathrm{c}=12.4504(6) \AA \quad \gamma=90^{\circ}$.

1237.33(10) $\AA^{3}$

2

$1.432 \mathrm{Mg} / \mathrm{m}^{3}$

$0.272 \mathrm{~mm}^{-1}$

552

$0.220 \times 0.160 \times 0.120 \mathrm{~mm}^{3}$

2.927 to $28.372^{\circ}$.

$-16<=\mathrm{h}<=16,-11<=\mathrm{k}<=11,-16<=\mathrm{l}<=16$

39510

$6182[\mathrm{R}(\mathrm{int})=0.0418]$

$99.9 \%$

Semi-empirical from equivalents

0.7457 and 0.7161

Full-matrix least-squares on $\mathrm{F}^{2}$

$6182 / 1 / 325$

0.912

$\mathrm{R} 1=0.0357, \mathrm{wR} 2=0.1039$

$\mathrm{R} 1=0.0495, \mathrm{wR} 2=0.1203$

0.014(16)

$\mathrm{n} / \mathrm{a}$

0.292 and -0.168 e. $\AA^{-3}$ 


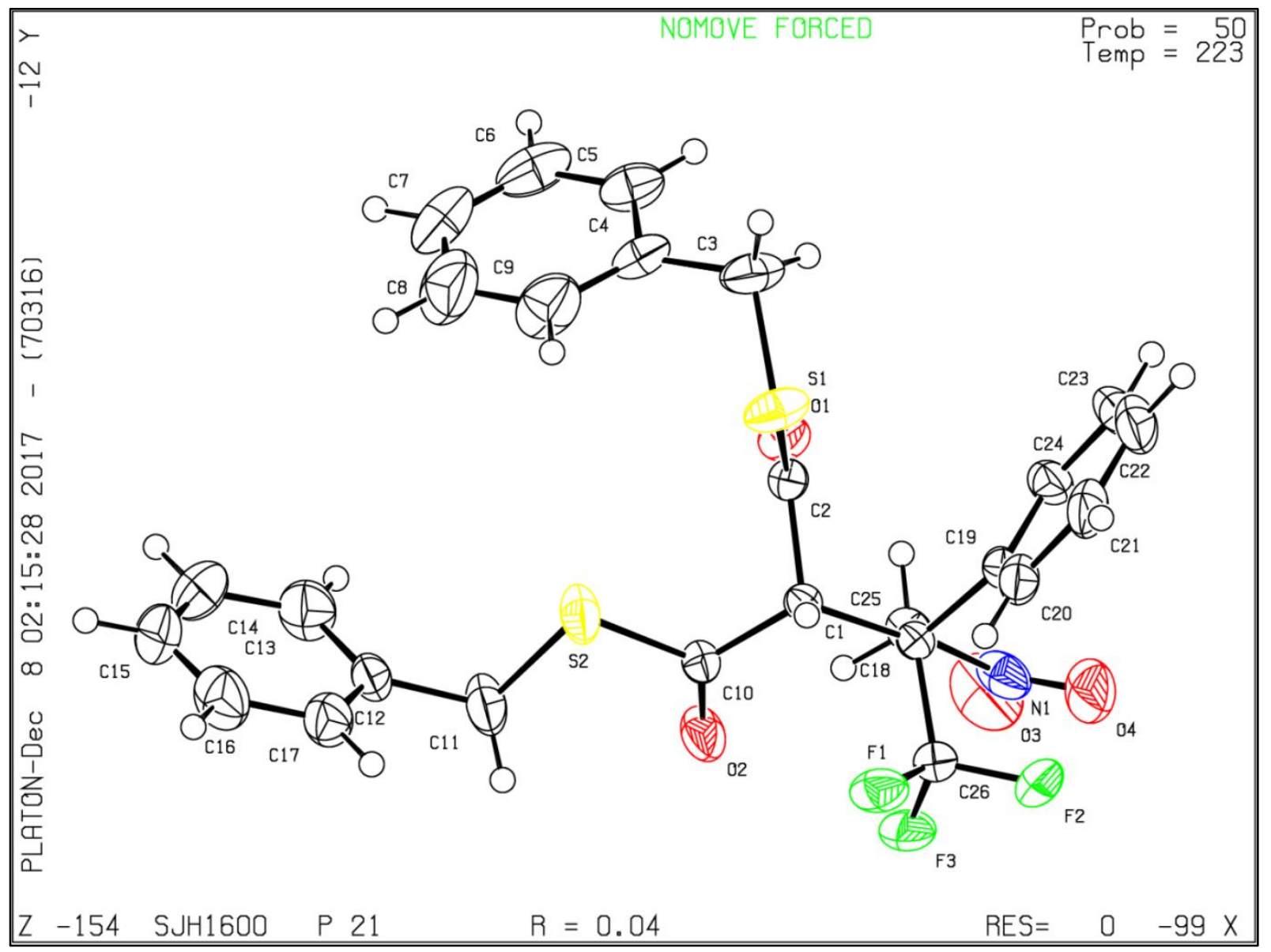

Figure S2. ORTEP structure of ent-3ab. 


\section{References}

(1) (a) G. A. Olah, T. Matthew, G. Rasul, M. Mandal, R. Kultyshev, V. Surampudi, H. Vaghoo, C. Panja, G. K. S. Prakash, J. Org. Chem. 2006, 71, 6806-6813; (b) J.-R. Gao, H. Wu, B. Xiang, W.B. Yu, L. Han, Y.-X. Jia, J. Am. Chem. Soc. 2013, 135, 2983-2986; (c) G. Cheng, B. Xia, Q. Wu, X. Lin, RSC Adv. 2013, 3, 9820-9828; (d) H. Cheng, Y. Pei, F. Leng, J. Li, A. Liang, D. Zou, Y. Wu, Y. Wu, Tetrahedron Lett. 2013, 54, 4483-4486; (e) F.-L. Liu, J.-R. Chen, B. Feng, X.-Q. Hu, L.-H. Ye, L.-Q. Lu, W.-J. Xiao, Org. Biomol. Chem. 2014, 12, 1057-1060.

(2) H. Y. Bae, M. J. Kim, J. H. Sim, C. E. Song, Angew. Chem. Int. Ed. 2016, 55, 10825-10829.

(3) (a) A. Berkessel, S. Mukherjee, T. N. Müller, F. Cleemann, K. Roland, M. Brandenburg, J.-M. Neudörfl, J. Lex, Org. Biomol. Chem. 2006, 4, 4319-4330; (b) M. Imanishi, Y. Tomishima, S. Itou, H. Hamashima, Y. Nakajima, K. Washizuka, M. Sakurai, S. Matsui, E. Imamura, K. Ueshima, T. Yamamoto, N. Yamamoto, H. Ishikawa, K. Nakano, N. Unami, K. Hamada, Y. Matsumura, F. Takamura, K. Hattori, J. Med. Chem. 2008, 51, 1925-1944; (c) T. Liu, D. Li, S. Wang, Y. Hu, X. Dong, X.-Y. Liu, C.-M. Che, Chem. Commun. 2014, 50, 13261-13264; (d) H. Liu, Y. He, J. Jiao, D. Bai, D. Chen, R. Krishna, B. Chen, Chem. Eur. J. 2016, 22, 14988-14997.

(4) H. E. Gottlieb, V. Kotlyar, A. Nudelman, J. Org. Chem. 1997, 62, 7512-7515.

(5) P. Kwiatkowski, A. Cholewiak, A. Kasztelan, Org. Lett. 2014, 16, 5930-5933. 